\title{
A perspective on light-induced transport of particles: from optical forces to phoretic motion
}

\author{
Pavel Zemánek ${ }^{1 *}$, Giorgio Volpe ${ }^{2}$, Alexandr Jonáš ${ }^{1}$ and Oto \\ BRZOBOHATÝ1 \\ ${ }^{1}$ Institute of Scientific Instruments of CAS, Czech Academy of Sciences, \\ Královopolská 147, 61264 Brno, Czech Republic \\ ${ }^{2}$ Department of Chemistry, University College London, 20 Gordon Street, London WC1H OAJ, United \\ Kingdom \\ *Corresponding author: zemanek@isibrno.cz
}

\begin{abstract}
:
Propulsive effects of light, which often remain unnoticed in our daily-life experience, manifest themselves on spatial scales ranging from subatomic to astronomical. Light-mediated forces can indeed confine individual atoms, cooling their effective temperature very close to absolute zero, as well as contribute to cosmological phenomena such as the formation of stellar planetary systems. In this review, we focus on the transport processes that light can initiate on small spatial scales. In particular, we discuss in depth various light-induced mechanisms for the controlled transport of microscopic particles; these mechanisms rely on the direct transfer of momentum between the particles and the incident light waves, on the combination of optical forces with external forces of other nature, and on light-triggered phoretic motion. After a concise theoretical overview of the physical origins of optical forces, we describe how these forces can be harnessed to guide particles either in continuous bulk media or in the proximity of a constraining interface under various configurations of the illuminating light beams (radiative, evanescent, or plasmonics fields). Subsequently, we introduce particle transport techniques that complement optical forces with counteracting forces of non-optical nature. We finally discuss particle actuation schemes where light acts as a fine knob to trigger and/or modulate phoretic motion in spatial gradients of non-optical (e.g., electric, chemical, or temperature) fields. We conclude by outlining possible future fundamental and applied directions for research in light-induced particle transport. We believe that this comprehensive review can inspire diverse, interdisciplinary scientific communities to devise novel, unorthodox ways of assembling and manipulating materials with light.
\end{abstract}

(C) 2019 Optical Society of America

\section{Contents}

1 Introduction 2

2 Physical origin of optical forces: a reminder 3

2.1 Optical forces on a microparticle . . . . . . . . . . . . . . . . 4

2.2 Optical forces on a nanoparticle . . . . . . . . . . . . . 5

3 Transport of particles with optical forces in continuous bulk media 9

3.1 Pushing and guiding of particles along the beam direction in 3-D . . . . . . . 9

3.1.1 Gaussian beams . . . . . . . . . . . . . . . . . . . 9

3.1 .2 Bessel beams . . . . . . . . . . . . . . . . . . . . . . . . . . . . . . . . .

3.1 .3 "Curved" beams ....................... . . 18

3.23 -D optical pulling in tractor beams . . . . . . . . . . . . . . . 22

3.3 Counter-propagating beams and optical conveyor belts . . . . . . . . . . 25 
3.3.1 Dual-beam geometry . . . . . . . . . . . . . . . . . . . 26

3.3.2 Standing waves and optical conveyor belts . . . . . . . . . . . 28

3.4 Other methods for particle transport in continuous bulk media . . . . . . . . . 31

4 Interface-constrained transport with optical forces 36

4.1 Incident radiative waves . . . . . . . . . . . . . . . . . . . . . 36

4.1.1 Planar liquid-solid interfaces . . . . . . . . . . . . . . . . . . 37

4.1 .2 Air-liquid interfaces . . . . . . . . . . . . . . . . . . 43

4.2 Transport based on optical waveguides . . . . . . . . . . . . . . . . 45

4.3 Transport based on optical fibers . . . . . . . . . . . . . . . . . . . . . . . . 49

4.3 .1 Liquid-fiber interfaces . . . . . . . . . . . . . . . . . . . . . . . 49

4.3.2 Gas-fiber interfaces . . . . . . . . . . . . . . . . . . 50

4.4 An interplay of forces of optical and non-optical nature . . . . . . . . . . 50

4.4 .1 Optical chromatography . . . . . . . . . . . . . . . 50

4.4.2 Transport of particles using the Magnus effect . . . . . . . . . . . 53

4.5 Transport with plasmonic fields . . . . . . . . . . . . . . . 55

5 Light-induced phoresis in non-optical fields $\quad 59$

5.1 Optically induced dielectrophoresis . . . . . . . . . . . . . . . . . . . . . . . . 59

5.2 Optically induced thermophoresis . . . . . . . . . . . . . . . . . 62

5.3 Photophoresis in absorbing particles $\ldots \ldots \ldots \ldots 6 \ldots$

5.4 Light-induced enhanced diffusion $\ldots \ldots \ldots$. . . . . . . . . . . . . 64

5.5 Propulsion by light-induced body deformation . . . . . . . . . . . . 66

5.6 Transport by optically induced capillary effects and Marangoni stresses . . . . 66

6 Conclusions and outlook

\section{Introduction}

Within the past five decades, light-induced confinement and transport of physical objects - once merely an interesting peculiarity and a popular attribute of science fiction books and movies has firmly established itself as a precise, versatile experimental technique with applications in a variety of research fields ranging from natural sciences, to engineering and medicine. For the field of optical manipulation, this successful path to the spotlight began in the early 1970's by a series of pioneering articles authored by Arthur Ashkin [1-3] and culminated in 2018, when he was awarded the Nobel Prize in Physics "for the optical tweezers and their application to biological systems" [4]. This outstanding scientific achievement followed two other Nobel Prizes in Physics that were previously awarded in 1997 for groundbreaking experiments exploiting the transfer of momentum carried by light for cooling of individual atoms [5] and in 2001 for forming Bose-Einstein condensates - macroscopic physical systems that display quantum coherence [6].

Since its inception, the actuation of microscopic particles by light has undergone a remarkable evolution. The first proof-of-principle demonstrations used the radiation pressure of a single loosely focused laser beam to accelerate and guide particles along the beam axis [1]. By employing two counter-propagating beams with mutually opposing radiation pressures, it was possible to confine the particles in all three dimensions [1]. The invention of the optical tweezers, which enabled full spatial confinement of micro- and nanoparticles with a single tightly focused laser beam [7], then represented a major breakthrough that eventually introduced light-based micromanipulation into research laboratories across diverse scientific disciplines. Gradually, the range of configurations of the incident light waves that could be harnessed to confine and transport the illuminated particles has been expanded to include evanescent fields created in the vicinity of material interfaces and inner and outer boundaries of optical waveguides [8,9], 
propagation-invariant light beams with various spatial profiles of optical intensity and phase generated by complex beam shaping [10,11], plasmonic fields of metallic nanostructures [12], and even illumination geometries known as "tractor beams" that enable pulling the particles against the incident photon flux $[13,14]$. In addition to relying solely on the direct transfer of momentum between the incident light waves and the illuminated microscopic particles, optical forces have been complemented by other external forces acting on the transported particles, such as gravity [2], hydrodynamic forces [15, 16], acoustic forces [17] and electric forces [18-20]. Selective light-induced particle guiding and sorting has been also achieved in the presence of a constraining interface [16,21]. Alternatively, the energy of the incident photons has been used as a means to actuate particles indirectly by inducing non-optical external forces on the target particles [22,23].

All these routes for light-driven material manipulation have offered significant versatility to researchers and engineers, so the list of research fields that have immensely benefited from these methods is long and impressive: it includes, for example, colloid and interface science [24-26], fluid mechanics and microfluidics [27-29], analytical and preparative chemistry and biochemistry [30-32], micro- and nanofabrication [33,34] as well as biophotonics and biomedicine [35-37]. Optical stimulation has also proved attractive for developing novel types of active self-propelling particles acting as non-equilibrium microscopic transducers of free energy $[38,39]$.

In this review, we aim to provide a comprehensive picture of the physical origins, specific implementations, and selected applications of different light-induced mechanisms for the controlled transport of particles in liquids and gases. We intend the review as a practical guide for the reader to navigate through the range of experimental tools that are currently available for the optical control of the motion of solid and liquid particles with sizes ranging from tens of nanometers up to a few millimeters. In Section 2, we present a condensed theoretical framework that describes the physical origins of the optical forces exerted on microparticles and nanoparticles by an optical field. Section 3 is dedicated to the guiding of particles with optical forces in continuous bulk media using different beam configurations, including Gaussian beams, propagation-invariant Bessel beams, accelerating or curved beams, tractor beams and non-interfering or interefering counter-propagating beams. Alternative methods for the optical delivery of particles in bulk media are summarized too. Section 4 covers the main experimental strategies in which light-induced transport of particles is assisted by a constraining interface, including those based on radiative waves, evanescent waves, and plasmonics fields in the proximity of liquid/solid interfaces. Strategies for particle actuation that can be employed in the vicinity of air/liquid and air/solid interfaces are also briefly mentioned. In addition, particle transport techniques that complement optical forces with counteracting forces of non-optical nature (in particular, hydrodynamic forces) are presented. Section 5 discusses experimental schemes where light triggers phoretic motion in non-optical fields associated with spatial gradients of electric potential, temperature, and concentration generated by light in the particle's surroundings. In addition, this section also introduces self-phoresis driven by light-induced asymmetry in the physical or chemical properties of the manipulated particles. Finally, Section 6 outlines possible future fundamental and applied directions for research in light-induced particle transport.

\section{Physical origin of optical forces: a reminder}

In this section, we lay the theoretical groundwork for understanding the methods of manipulation and transport of small particles that are based on the direct exchange of momentum between the incident photons and the irradiated object located either in the bulk of a continuous host medium or at the interface between two different media. This type of light-matter interaction has been intensively studied since the 1970's when Arthur Ashkin published his first papers on the behavior of microparticles immersed in a liquid medium and illuminated by a laser 
beam $[1,40]$. Since Ashkin's seminal work, forces generated by light have been exploited to develop many useful devices. These achievements have been summarized in numerous general review articles [9, 12,21,29,30,41-72] and books [73-83] and include optical tweezers [7, 50,73] for the 3-D manipulation of nano- and micro-objects using tightly focused laser beams, optical stretchers for stretching deformable artificial microobjects or living cells and characterizing their elastic properties [84,85], holographic optical tweezers enabling the simultaneous independent manipulation of tens of microobjects [86-89], iTweezers using an iPad to view and manipulate microparticles [90], optical grippers for manipulating a selected object with the help of several optically trapped microscopic handles surrounding the target object [91,92], various types of optical tweezers exploiting user interfaces coming from the computer gaming industry (e.g., 3-D joysticks and haptic devices [91,93], Kinect technology [94-96], and sensors tracking hands or gaze and accepting voice commands [97]), plasmonic tweezers employing plasmonic resonances to increase the near-field intensity in the optical trap [12,98-101], optical pico-tensiometers measuring the tiny forces in the range from tenths to hundreds of piconewtons $(\mathrm{pN})$ associated with molecular motors $[53,57,58]$, optical sorters for separating objects with different physical properties upon illumination of the mixture [16,102-106], optical conveyor belts for delivering objects bidirectionally in spatially structured laser beams [14,107-109], and optical tractor beams pulling objects against the incident photon flux [13,110-112].

Here, we will not present a detailed theoretical description of the calculation of optical forces (covered, for example, in Refs. [78, 79, 81, 113]); instead, we will introduce limiting-case theoretical approaches based on ray optics and induced dipoles that offer a more intuitive picture of the origin of optical forces and their influence on the behavior of illuminated particles. In particular, the ray optics approximation is justified if the particle diameter is much larger than the trapping light wavelength and acceptable quantitative results can be obtained for objects of diameters in units of micrometers $[81,114,115]$. On the other hand, treating the manipulated particles as elementary induced dipoles is possible within the framework of Rayleigh scattering approximation that holds well for objects with characteristic dimensions sufficiently smaller than the trapping light wavelength, typically less than a few tens of nanometers $[116,117]$.

\subsection{Optical forces on a microparticle}

An elementary explanation of the optical forces acting upon a reflecting, absorbing or refracting object is illustrated in Fig. 1. If such an object is illuminated by a bundle of light rays, the incident photons are reflected, absorbed and refracted by the object. Following Newton's second law, the rate of change of the photon momentum is associated with the force acting on the photons. Consequently, a force of the same magnitude and opposite direction is applied to the irradiated object by virtue of Newton's third law of action and reaction. The force due to single-photon scattering has a noticeable effect only on a tiny object with a sufficiently small mass and momentum, such as a cold atom. In order to influence the behavior of bigger objects with sizes up to tens of micrometers, the coordinated action of many photons is required. This can only be achieved with an intense light field such as that of a focused laser beam. Obviously, absorption of the trapping light by the particle (and also by the surrounding medium) should be low to avoid excessive heating of the sample. In general, optical forces are linearly proportional to the total laser power $P$ incident on the object and can be expressed as $\mathbf{F}_{\text {opt }}=n_{\mathrm{m}} P \mathbf{Q} / c$, where $n_{\mathrm{m}}$ is the refractive index of the medium surrounding the object, $c$ is the speed of light in vacuum, and $\mathbf{Q}$ is the vectorial trapping efficiency factor that depends on the size, shape, material, and position of the particle, as well as on the wavelength and the spatial profiles of the beam intensity and phase. $\mathbf{Q}$ reaches its maximal absolute value $(|\mathbf{Q}|=2)$ in the case of purely back-reflected normally-incident light (for example, from a $100 \%$ reflective mirror), corresponding to the maximal transfer of momentum from the photons to the particle. However, its typical value encountered in optical micromanipulation experiments is $|Q| \simeq 0.1$. In comparison with the 
case of nanoparticles discussed in the next subsection, the nomenclature of individual force components in the ray-optics regime is not fully rigorous; the force pushing the particle along (or pulling it against) the direction of the Poynting vector of the incident light is usually called the scattering pushing (or pulling) force, whereas the force acting in the direction of the axial or lateral gradients of optical intensity is usually called the gradient force [114]. In some cases, the maximal optical force can reach hundreds of $\mathrm{pN}$, but in the majority of experiments, it is in the range from tenths to tens of $\mathrm{pN}$.

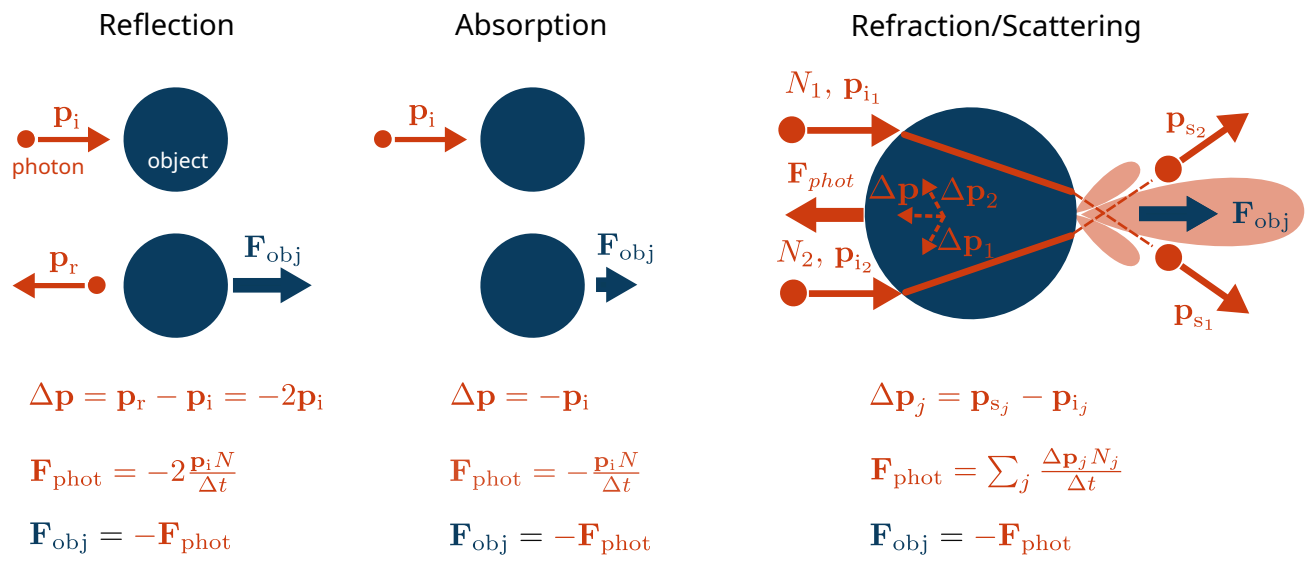

Fig. 1. An elementary explanation of the optical forces exerted on a reflecting, absorbing and refracting object. In the case of reflection, let us assume normal incidence of light and $100 \%$ reflectivity of the object's surface, with all $N$ photons incident on the object reflected back. Each incident and reflected photon carries a linear momentum $\mathbf{p}_{\mathrm{i}}=\hbar \mathbf{k}_{\mathrm{i}}$ and $\mathbf{p}_{\mathrm{r}}=\hbar \mathbf{k}_{\mathrm{r}}$, respectively, where $\mathbf{k}$ denotes the wavevector, $\hbar=h / 2 \pi$, and $h$ is the Planck constant. Following Newton's second law, the net force $\mathbf{F}_{\text {phot }}$ acting upon the photons due to the interaction with the object is proportional to the rate of change of linear momentum of all photons: $\mathbf{F}_{\text {phot }}=\left(\mathbf{p}_{\mathrm{r}}-\mathbf{p}_{\mathrm{i}}\right) N / \Delta t$. Following Newton's third law, the reaction force acting upon the object is then equal to $\mathbf{F}_{\text {obj }}=-\mathbf{F}_{\text {phot }}$. Similar considerations apply to the case of absorption, assuming for simplicity $100 \%$ object absorption. Under these idealized conditions, the optical force acting upon the reflecting object is two times larger than the force acting on the absorbing object. In the case of refraction/scattering, let us assume the wavefront of the incident beam can be divided into small area segments $A_{j}$ corresponding to individual rays within the beam. The power $P_{j}$ of each ray can be then expressed as $P_{j}=|\langle\mathbf{S}\rangle| A_{j}$, where $\langle\mathbf{S}\rangle$ is the time-averaged Poynting vector at the location of $A_{j}$ and the ray propagates along the direction of $\langle\mathbf{S}\rangle$, perpendicularly to $A_{j}$. Thus, along the $j^{\text {th }}$ ray and within a time $\Delta t, N_{j}=P_{j} \Delta t /(\hbar \omega)$ photons carry energy $P_{j} \Delta t$, incident momentum $\mathbf{p}_{\mathrm{i}_{j}}$ and scattered momentum $\mathbf{p}_{\mathrm{s}_{j}}$, with $\omega$ being the angular frequency of the light. The total optical force $\mathbf{F}_{\text {obj }}$ acting upon the object is then obtained as the sum of the force contributions from all rays in the beam. The schematics inside the object illustrates the change of photon momentum $\Delta \mathbf{p}$ coming from the two representative rays 1 and 2 (see $[81,114,115]$ for more details).

\subsection{Optical forces on a nanoparticle}

Since a nanoparticle is much smaller than the wavelength of visible or near-infrared light typically used in optical micromanipulations, the above described ray optics approach cannot be applied. Instead, an illuminated nanoparticle is frequently treated as an elementary induced electric dipole subjected to the Lorentz force in an incident electromagnetic (EM) field, which leads to the 
following equation for the time-averaged force acting upon the nanoparticle [116]:

$$
\left\langle F_{i}\right\rangle=\frac{1}{T} \int_{-T / 2}^{T / 2} \sum_{j=x, y, z} \bar{d}_{j} \frac{\partial \bar{E}_{i}}{\partial r_{j}} \mathrm{~d} t+\frac{1}{T} \int_{-T / 2}^{T / 2} \sum_{j, k=x, y, z} \varepsilon_{i j k} \frac{\partial \bar{d}_{j}}{\partial t} \bar{B}_{k} \mathrm{~d} t,
$$

where $T$ is the period of the time-harmonic EM field, $\bar{d}_{k}, \bar{E}_{k}, \bar{B}_{k}$ are the real-valued $k^{t h}$ components of the time-harmonic dipole $\overline{\mathbf{d}}(\mathbf{r}, t)=\mathfrak{R}\{\mathbf{d}(\mathbf{r}) \exp (-\mathrm{i} \omega t)\}$, electric field $\overline{\mathbf{E}}(\mathbf{r}, t)=$ $\mathfrak{R}\{\mathbf{E}(\mathbf{r}) \exp (-\mathrm{i} \omega t)\}$, and magnetic field $\overline{\mathbf{B}}(\mathbf{r}, t)=\mathfrak{R}\{\mathbf{B}(\mathbf{r}) \exp (-\mathrm{i} \omega t)\}$, respectively, $\mathfrak{R}\{\}$ denotes the real part of the quantity in the brackets, $r_{j}$ are the spatial coordinates, and $\varepsilon_{i j k}$ denotes the Levi-Civita tensor. Indices $i, j, k$ then run through the $x, y$ and $z$ components of the vectors.

Using Maxwell's equations for the complex amplitudes of the EM field vectors and the standard vector calculus identities, Eq. (1) then gives [81,116,118,119]:

$$
\left\langle F_{i}\right\rangle \equiv F_{i}=\frac{1}{2} \mathfrak{R}\left\{\sum_{j=x, y, z} d_{j} \frac{\partial E_{j}^{*}}{\partial r_{i}}\right\}, \text { where } d_{j}=\varepsilon_{\mathrm{m}} \varepsilon_{0} \alpha E_{j} .
$$

In Eq. (2), time averaging of the harmonically varying quantities on the right-hand side has been carried out and the asterisk * denotes the complex-conjugated quantity. $d_{j}$ is the $j^{\text {th }}$ component of the induced dipole following Bohren's notation [117], $\varepsilon_{0}$ is the permittivity of vacuum, $\varepsilon_{\mathrm{m}} \equiv n_{\mathrm{m}}^{2}$ is the relative permittivity of the medium surrounding the particle with $n_{\mathrm{m}}$ being the refractive index of the medium, and $\alpha$ is the polarizability of the particle. In general, the polarizability $\alpha$ has a real and an imaginary part $\alpha^{\prime}$ and $\alpha^{\prime \prime}$, respectively; thus Eq. (2) can be rewritten in component or vector form as $[81,116,118,119]$ :

$$
\begin{aligned}
\frac{F_{i}}{\varepsilon_{0} \varepsilon_{\mathrm{m}}} & =\frac{1}{4} \alpha^{\prime} \frac{\partial\left(E_{j} E_{j}^{*}\right)}{\partial r_{i}}-\frac{1}{2} \alpha^{\prime \prime} \mathfrak{T}\left\{\varepsilon_{i j k} E_{j} \varepsilon_{k l n} \frac{\partial E_{n}^{*}}{\partial r_{l}}\right\}-\frac{1}{2} \alpha^{\prime \prime} \mathfrak{T}\left\{E_{j} \frac{\partial E_{i}^{*}}{\partial r_{j}}\right\}, \\
\frac{\mathbf{F}}{\varepsilon_{0} \varepsilon_{\mathrm{m}}} & =\frac{1}{4} \alpha^{\prime} \nabla|\mathbf{E}|^{2}-\frac{1}{2} \alpha^{\prime \prime} \mathfrak{I}\left\{\mathbf{E} \times\left(\nabla \times \mathbf{E}^{*}\right)\right\}-\frac{1}{2} \alpha^{\prime \prime} \mathfrak{J}\left\{(\mathbf{E} \cdot \nabla) \mathbf{E}^{*}\right\},
\end{aligned}
$$

where $|\mathbf{X}|=\sqrt{\mathbf{X} \cdot \mathbf{X}^{*}}$ and $\mathfrak{I}\{\mathbf{X}\}$ denote the absolute value and the imaginary part of the complex vector $\mathbf{X}$, respectively. This expression can be further rewritten using Maxwell's equations $\left(\nabla \times \mathbf{E}^{*}=-\mathrm{i} \omega \mu_{0} \mathbf{H}^{*}, \nabla \cdot \mathbf{E}=0\right)$, the vector calculus identities, the time averaged Poynting vector $\mathbf{S}$ and the spin density of transverse electromagnetic field $\mathbf{L}_{\mathbf{s}}$ in the following vector form $[118,120]$ :

$$
\begin{aligned}
\mathbf{F} & =\frac{\varepsilon_{0} \varepsilon_{\mathrm{m}}}{4} \alpha^{\prime} \nabla|\mathbf{E}|^{2}+\frac{\varepsilon_{\mathrm{m}}}{2} \frac{k_{0}}{c} \alpha^{\prime \prime} \mathfrak{R}\left\{\mathbf{E} \times \mathbf{H}^{*}\right\}+\frac{\varepsilon_{0} \varepsilon_{\mathrm{m}}}{2} \alpha^{\prime \prime} \nabla \times \mathfrak{T}\left\{\mathbf{E} \times \mathbf{E}^{*}\right\} \\
& =\frac{\varepsilon_{0} \varepsilon_{\mathrm{m}}}{4} \alpha^{\prime} \nabla|\mathbf{E}|^{2}+\frac{\varepsilon_{\mathrm{m}} k_{0}}{c} \alpha^{\prime \prime} \mathbf{S}+\varepsilon_{\mathrm{m}} \omega \alpha^{\prime \prime} \nabla \times \mathbf{L}_{\mathrm{s}}, \\
\mathbf{L}_{\mathrm{s}} & =\frac{\varepsilon_{0}}{4 \mathrm{i} \omega}\left(\mathbf{E} \times \mathbf{E}^{*}\right), \quad \mathbf{S}=\frac{1}{2} \mathfrak{R}\left\{\mathbf{E} \times \mathbf{H}^{*}\right\} .
\end{aligned}
$$

The imaginary part of the polarizability $\alpha^{\prime \prime}$ is associated with the particle absorption (for example, in metals) or with the interaction of the induced dipole with itself through the scattered light [121]. Thus, the polarizability at the optical frequencies can be written in the form:

$$
\alpha=\alpha^{\prime}+\mathrm{i} \alpha^{\prime \prime}=\frac{\alpha_{0}}{1-\frac{\mathrm{i}}{6 \pi} k^{3} \alpha_{0}} \simeq \alpha_{0}^{\prime}+\mathrm{i} \alpha_{0}^{\prime \prime}+\mathrm{i} \frac{\left|\alpha_{0}\right|^{2} k^{3}}{6 \pi},
$$

where $k=2 \pi n_{\mathrm{m}} / \lambda_{0}=n_{\mathrm{m}} k_{0}, \lambda_{0}$ denotes the vacuum wavelength, and the value of $\alpha_{0}$ for a spherical particle with radius $a$ can be expressed using the Lorentz-Lorenz relation as:

$$
\alpha_{0} \equiv \alpha_{0}^{\prime}+\mathrm{i} \alpha_{0}^{\prime \prime}=4 \pi a^{3} \frac{\varepsilon_{\mathrm{p}}-\varepsilon_{\mathrm{m}}}{\varepsilon_{\mathrm{p}}+2 \varepsilon_{\mathrm{m}}}=4 \pi a^{3} \frac{\varepsilon_{\mathrm{p}}^{\prime}+\mathrm{i} \varepsilon_{\mathrm{p}}^{\prime \prime}-\varepsilon_{\mathrm{m}}}{\varepsilon_{\mathrm{p}}^{\prime}+\mathrm{i} \varepsilon_{\mathrm{p}}^{\prime \prime}+2 \varepsilon_{\mathrm{m}}},
$$


where $\varepsilon_{\mathrm{p}} \equiv n_{\mathrm{p}}^{2}$ is the relative permittivity of the particle, generally complex for an absorbing particle, and $n_{\mathrm{p}}$ is its refractive index. Following Bohren [117], Eq. (7) can be rewritten using the cross-sections for absorption $C_{\mathrm{abs}}=k \alpha_{0}^{\prime \prime}$, scattering $C_{\mathrm{sca}}=\left|\alpha_{0}\right|^{2} k^{4} /(6 \pi)$, and extinction $C_{\text {ext }}=C_{\mathrm{abs}}+C_{\mathrm{sca}}$ :

$$
\alpha \simeq \alpha_{0}^{\prime}+\mathrm{i} \frac{C_{\text {abs }}}{k}+\mathrm{i} \frac{C_{\text {sca }}}{k}=\alpha_{0}^{\prime}+\mathrm{i} \frac{C_{\text {ext }}}{k} .
$$

Using Eq. (9) and Eq. (5), we can write the final expression for the optical force acting upon a nanoparticle as:

$$
\mathbf{F}=\frac{1}{4} \varepsilon_{0} \varepsilon_{\mathrm{m}} \alpha^{\prime} \nabla|\mathbf{E}|^{2}+C_{\mathrm{ext}} \frac{n_{\mathrm{m}}}{c} \mathbf{S}+C_{\mathrm{ext}} c n_{\mathrm{m}} \nabla \times \mathbf{L}_{\mathrm{s}}
$$

Let us stress that, in all the above presented equations, only the unperturbed fields incident on the particle are considered. Furthermore, it is also assumed that the nanoparticle represented by an induced dipole scatters light symmetrically with respect to the polar angle $\theta$; thus, instead of the full cross-section for the radiation pressure $C_{\mathrm{pr}}=C_{\mathrm{ext}}-\langle\cos (\theta)\rangle C_{\mathrm{sca}}, C_{\mathrm{ext}}$ alone can be used, as the asymmetry parameter $\langle\cos (\theta)\rangle=0$ in this case [117].

The first term in Eq. (10) is known as the gradient force $\mathbf{F}_{\mathrm{gr}}$, because it is proportional to the gradient of the squared magnitude of the electric field. For a nanoparticle illuminated by a transverse electromagnetic wave (for example, a laser beam), this term can be expressed using the optical intensity, or irradiance, $I=n_{\mathrm{m}} \varepsilon_{0} c|\mathbf{E}|^{2} / 2$ of the wave as:

$$
\mathbf{F}_{\mathrm{gr}}=\frac{n_{\mathrm{m}}}{2 c} \alpha^{\prime} \nabla I
$$

The gradient force is conservative and pulls the particle to the location of the maximal field intensity if $\alpha^{\prime}>0$, i.e., if the refractive index of the particle is higher than the refractive index of the surrounding medium $\left(n_{\mathrm{p}}>n_{\mathrm{m}}\right)$. In the case of $\alpha^{\prime}<0\left(n_{\mathrm{p}}<n_{\mathrm{m}}\right)$, the particle is repelled to the low-intensity region of the beam. Figure 2 a illustrates the spatial profile of this force.

The second term in Eq. (10) describes the scattering force $\mathbf{F}_{\mathrm{sc}}$ (also known as the radiation pressure), which points in the direction of the Poynting vector, i.e., along the energy flux density:

$$
\mathbf{F}_{\mathrm{sc}}=C_{\mathrm{ext}} \frac{n_{\mathrm{m}}}{c} \mathbf{S}
$$

Regardless of the ratio of refractive indices of the particle and the surrounding medium, the particle is always pushed in the direction of $\mathbf{S}$. Figure $2 \mathrm{~b}$ illustrates the physical origin and the spatial profile of this non-conservative force.

The last term in Eq. (10) then describes the non-conservative spin-curl force, which arises from the polarization gradients in the incident field [81,118]. Since it is usually much smaller than the scattering and gradient force, we will not consider it further. 

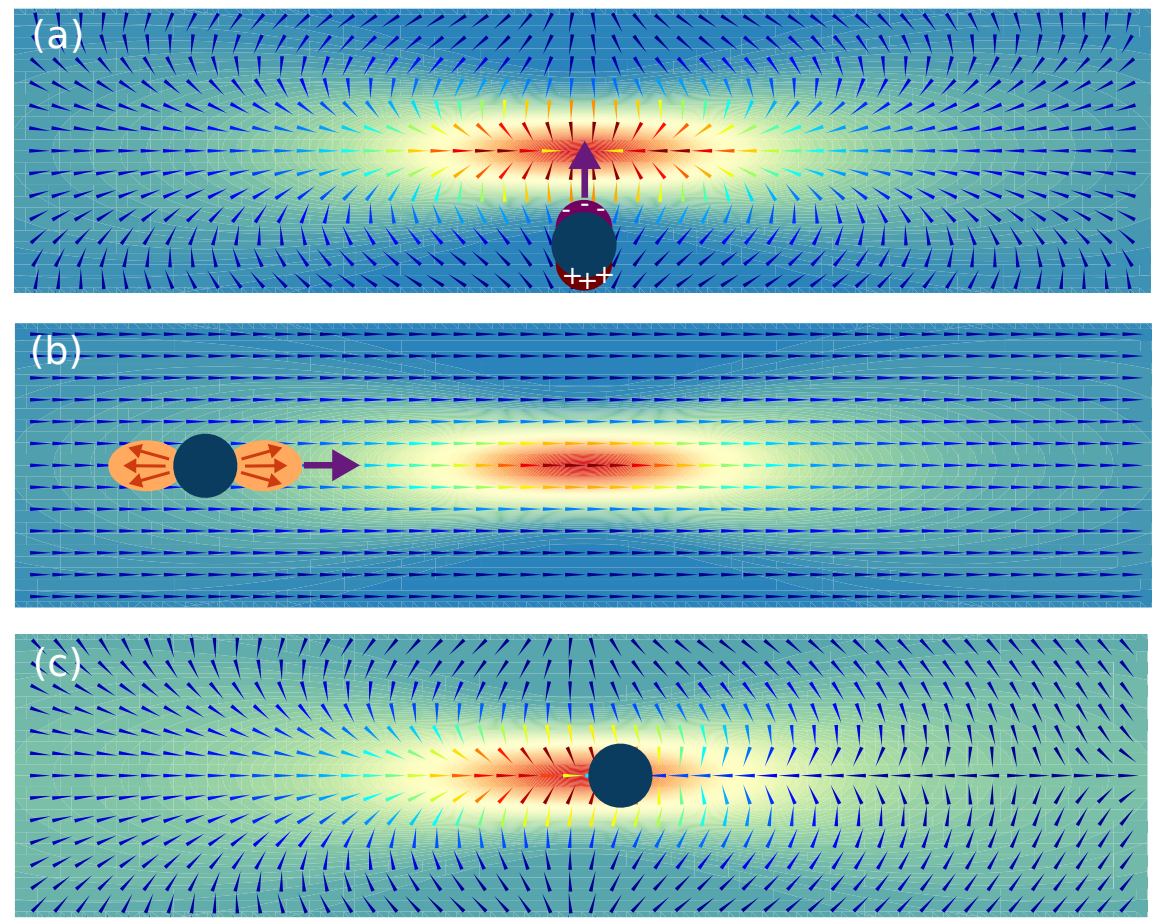

Fig. 2. Elementary explanation of the optical forces acting upon a nanoparticle with $n_{\mathrm{p}}>n_{\mathrm{m}}$ illuminated with a focused Gaussian laser beam incident from the left. (a) Illustration of the gradient force $\mathbf{F}_{\text {gr }}$ pointing to the high-intensity region of the beam (red) as a result of the behavior of an induced electric dipole placed into an inhomogeneous electromagnetic field. (b) Illustration of the scattering force $\mathbf{F}_{\mathrm{sc}}$ resulting from the change of linear momentum of the photons incident on the object from a single direction and scattered by the object in all directions. (c) Illustration of the total optical force acting upon the nanoparticle, obtained as the sum of the gradient and scattering forces. Due to the pushing effect of the scattering force, the equilibrium position of the nanoparticle is located slightly behind the beam focus (in the beam propagation direction). 


\section{Transport of particles with optical forces in continuous bulk media}

This section deals with the transport of particles that are surrounded by a gaseous or liquid medium and confined and propelled solely by optical forces. Figure 1 shows that if an illuminated object reflects, absorbs, or scatters incident light away from its original direction of propagation, the resulting radiation pressure pushes the object along this direction. Probably the oldest mention of this phenomenon in the scientific literature can be found in the astronomical observations of Johannes Kepler and is related to the shape and direction of comets' dust tails. According to Kepler's proposal, it is the radiation pressure of sunlight that pushes the dust particles in the comets' tails away from the Sun [122]. The same principle has been recently tested as an alternative mechanism for propelling manmade satellites equipped with extremely thin, highly reflective solar sails [123-126]. Coincidentally, the solar light pressure was also utilized to control the pointing of the spacecraft Kepler, which - while looking for Earth-like exo-planets - suffered a malfunction of two of its stabilization gyroscopes [127, 128]. However, the low intensity of solar illumination can only provide a weak pushing force. In the case of the solar sail Ikaros launched by The Japan Aerospace Exploration Agency, the measured force acting upon its $196 \mathrm{~m}^{2}$ sail was merely $1.12 \mathrm{mN}$ [125]. In order to achieve stronger propulsion forces, the Breakthrough Starshot Initiative proposed to use a phased laser array placed on Earth [129]. This laser array would target a nano-spacecraft with an ultra-thin sail of mass about $1 \mathrm{~g}$ and area 10 $\mathrm{m}^{2}$ and accelerate it to relativistic speeds of up to $0.2 c$ needed for a space travel towards Alpha Centauri.

Clearly, the requirements that have to be met by light sails usable for practical space explorations - in particular, low mass, high reflectivity, high structural integrity and resistance to mechanical damage by interplanetary dust - pose major technological challenges for the production and handling of the sails $[130,131]$. Fortunately, laboratory-scale experiments with controlled transport of small particles initiated by the forces of light can be readily performed on a table top using currently available technology. As mentioned above, radiation pressure resulting from illumination of objects with light of intensity comparable to that of solar radiation on the surface of the Earth is rather small. For a microparticle with a cross-sectional area of $1 \mu \mathrm{m}^{2}$, the net optical force is on the order of $10^{-18} \mathrm{~N}$, much smaller than the typical force due to gravity acting on the same particle. Therefore, in order to achieve more significant forces, experiments carried out at the microscale usually rely on focused or structured laser beams [1].

\subsection{Pushing and guiding of particles along the beam direction in 3-D}

The directed guiding of an object with optical forces involves the pushing of the object along the beam propagation direction and, simultaneously, its confinement in the lateral direction. In this subsection, we will focus on those beam geometries, in which the object is laterally confined by the beam itself and, thus, the lateral optical force $F_{r \text {,opt }}(\mathbf{r})$ is stronger than any other external force (e.g., gravity or stochastic thermal force) acting on the particle. In the case of an optically trapped nanoparticle, thermal activation is the leading mechanism of particle's escape from the potential well formed by the illuminating light beam. In order to keep the particle laterally confined, the work $W_{\text {lateral }}$ needed to remove the particle from the beam in the lateral direction must be much larger than the thermal energy:

$$
W_{\text {lateral }}=-\int_{0}^{\infty} F_{r, \text { opt }}(\mathbf{r}) \mathrm{d} r \gg k_{\mathrm{B}} T,
$$

where $k_{\mathrm{B}}$ is the Boltzman constant and $T$ is the absolute temperature.

\subsubsection{Gaussian beams}

In order to demonstrate the typical approach to the analysis of optical forces acting on a particle illuminated by a laser beam, let us consider first the archetypal example of a paraxial Gaussian 
(a)

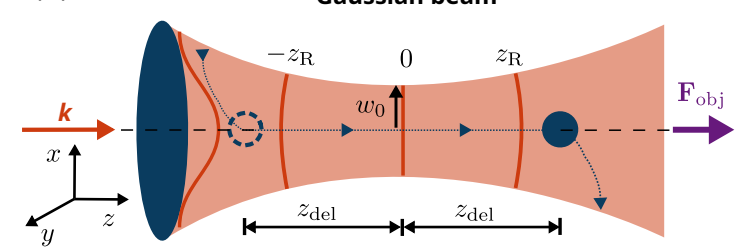

(b)

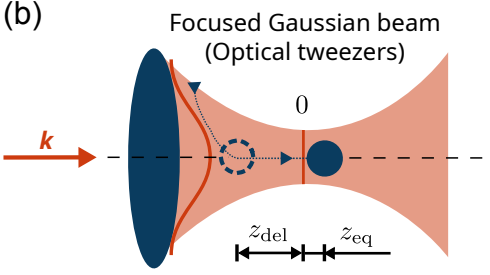

Fig. 3. Schematic illustration of the effects of a Gaussian beam upon an object illuminated by the beam. (a) A weakly focused Gaussian beam propagating from the left attracts a particle of refractive index higher than the surrounding medium towards the high-intensity region on the beam axis and, simultaneously, pushes the particle along the beam axis through the beam focus. At the maximal delivery distance $z_{\text {del }}$ behind the focus, the particle escapes laterally either due to gravity (for larger objects and horizontal beam propagation) or due to stochastic thermal forces (for smaller objects). (b) A tightly focused Gaussian beam provides a sufficiently strong longitudinal intensity gradient (generating the gradient force) to overcome the scattering (pushing) force. Thus, an optical tweezers is formed, allowing the particle to be trapped at the equilibrium distance $z_{\mathrm{eq}}$ behind the beam focus [7]. Dashed empty circles indicate the initial position of the particle in the beam, solid full circles indicate the final position of the particle in the beam.

beam (i.e., the TEM 00 mode with a Gaussian lateral intensity profile [132], see Fig. 3) polarized along the $x$ axis. The electric field $E_{x}$ of such a beam depends on the radial and axial coordinates $(r, z)$ as:

$$
E_{x}(r, z)=E_{0} \frac{w_{0}}{w(z)} \exp \left(-\frac{r^{2}}{w(z)^{2}}\right) \exp \left[\mathrm{i} k z+\frac{\mathrm{i} k r^{2}}{2 R(z)}-\mathrm{i} \arctan \left(\frac{z}{z_{\mathrm{R}}}\right)\right],
$$

where $r^{2}=x^{2}+y^{2}, w(z)=w_{0} \sqrt{1+z^{2} / z_{\mathrm{R}}^{2}}$ is the beam width, $R(z)=z\left(1+z_{\mathrm{R}}^{2} / z^{2}\right)$ is the radius of curvature of the beam wavefront, $z_{\mathrm{R}}=\pi w_{0}^{2} / \lambda$ is the Rayleigh length, $w_{0}$ is the beam waist and $\lambda=\lambda_{0} / n_{\mathrm{m}}$ is the light wavelength in the medium, with $\lambda_{0}$ being the vacuum wavelength. If such a beam of total power $P$ illuminates a non-absorbing nanoparticle that is optically denser than the surrounding medium (i.e., $n_{\mathrm{p}}>n_{\mathrm{m}}$ ), the scattering and gradient forces acting on the particle can be expressed analytically using Eq. (10) as [133,134]:

$$
\begin{aligned}
F_{z}(r, z) & =F_{z, \text { scat }}(r, z)+F_{z, \text { grad }}(r, z), \\
F_{z, \text { scat }}(r, z) & =F_{z, \text { scat }}^{\text {amp }} \frac{w_{0}^{2}}{w(z)^{2}} \exp \left(-\frac{2 r^{2}}{w(z)^{2}}\right), \\
F_{z, \text { grad }}(r, z) & =-F_{z, \text { grad }}^{\text {amp }} \frac{16 w_{0}^{4}}{9 \sqrt{3} z_{\mathrm{R}}} \frac{z}{w(z)^{4}}\left(1-\frac{2 r^{2}}{w(z)^{2}}\right) \exp \left(-\frac{2 r^{2}}{w(z)^{2}}\right), \\
F_{r, \text { grad }}(r, z) & =-F_{r, \text { grad }}^{\text {amp }} 2 \sqrt{e} w_{0}^{3} \frac{r}{w(z)^{4}} \exp \left(-\frac{2 r^{2}}{w(z)^{2}}\right),
\end{aligned}
$$


where $e \equiv \exp (1)$ and the force amplitudes $F_{z, \text { scat }}^{\mathrm{amp}}, F_{z, \text { grad }}^{\mathrm{amp}}$ and $F_{r, \text { grad }}^{\mathrm{amp}}$ correspond to the maximal values of the forces:

$$
\begin{aligned}
& F_{z, \text { scat }}^{\mathrm{amp}} \equiv F_{z, \text { scat }}(0,0)=\frac{16}{3} \frac{n_{\mathrm{m}}}{c} k^{4} a^{6} P\left(\frac{m^{2}-1}{m^{2}+2}\right)^{2} \frac{1}{w_{0}^{2}}, \\
& F_{z, \text { grad }}^{\mathrm{amp}} \equiv F_{z, \text { grad }}\left(0, z_{\mathrm{R}} / \sqrt{3}\right)=\frac{8 n_{\mathrm{m}}}{c} \frac{a^{3} P w_{0}^{2}}{z_{\mathrm{R}}^{2}}\left(\frac{m^{2}-1}{m^{2}+2}\right) \frac{9 \sqrt{3} z_{\mathrm{R}}}{16 w_{0}^{4}}, \\
& F_{r, \text { grad }}^{\mathrm{amp}} \equiv F_{r, \text { grad }}\left(w_{0} / 2,0\right)=\frac{16 n_{\mathrm{m}}}{c} a^{3} P\left(\frac{m^{2}-1}{m^{2}+2}\right) \frac{1}{2 \sqrt{e} w_{0}^{3}}
\end{aligned}
$$

where $m=n_{\mathrm{p}} / n_{\mathrm{m}}$ represents the relative refractive index of the particle.

Analysis of these equations give us an insight into the physical mechanism behind the optical forces acting in laser beams. The ratio

$$
\frac{F_{z, \text { scat }}^{\mathrm{amp}}}{F_{r, \text { grad }}^{\mathrm{amp}}}=\frac{2 \sqrt{e}}{3}(2 \pi)^{4}\left(\frac{m^{2}-1}{m^{2}+2}\right) \frac{w_{0}}{\lambda}\left(\frac{a}{\lambda}\right)^{3}
$$

reveals that the pushing axial force $F_{z, \text { scat }}^{\mathrm{amp}}$ increases with respect to the lateral gradient force $F_{r, \text { grad }}^{\text {amp }}$ with increasing beam waist size and particle radius. For tiny particles $(a / \lambda \ll 1)$, it then follows that $F_{r, \text { grad }}^{\mathrm{amp}}$ is much stronger than $F_{z \text {,scat }}^{\mathrm{amp}}$ and, consequently, the particle quickly moves to the beam axis.

In the axial direction, the two force components $F_{z \text {,scat }}$ and $F_{z \text {,grad }}$ compete with each other: while $F_{z \text {,scat }}$ pushes the particle along the $z$ axis, $F_{z \text {,grad }}$ pulls the particle towards the beam waist position at $z=0$. If the scattering force is stronger than the gradient force everywhere along the beam axis, i.e.,

$$
F_{z \text {,scat }}(0, z)+F_{z, \operatorname{grad}}(0, z)>0
$$

the nanoparticle is pushed past the position of the maximal longitudinal gradient force $F_{z \text {,grad }}$ at $r=0$ and $z=z_{\mathrm{R}} / \sqrt{3}$ and, thus, the particle cannot be confined along the beam axis (see Fig. 3a). In the opposite case, the so called optical tweezers is formed [7] and an equilibrium longitudinal position $z_{\mathrm{eq}}>0$ exists, at which the net axial force $F_{z}\left(0, z_{\mathrm{eq}}\right)=0$ and the slope of $F_{z}(0, z)$ with respect to $z$ is negative (Fig. 3b).

Since the beam intensity and width change along the $z$-axis, the particle can be guided by the beam only over a limited longitudinal range, within which the lateral gradient force $F_{r \text {,grad }}$ is sufficiently strong to confine the particle in the lateral direction. As the particle moves farther away from the beam waist position at $z=0$, the work $W_{r \text {,grad }}(z)$ needed to release the particle in the lateral direction decreases as:

$$
W_{r, \operatorname{grad}}(z)=-\int_{0}^{\infty} F_{r, \operatorname{grad}}(r, z) \mathrm{d} r=F_{r, \text { grad }}^{\mathrm{amp}} \frac{\sqrt{e} w_{0}}{2} \frac{1}{1+\left(z / z_{\mathrm{R}}\right)^{2}} .
$$

When $W_{r, \text { grad }}(z) \approx k_{\mathrm{B}} T$, the particle can be released from the beam by random thermal fluctuations. Under this condition, the maximal longitudinal delivery distance $z_{\text {del }}$ from the beam waist can be expressed as:

$$
z_{\text {del }}=z_{\mathrm{R}} \sqrt{\frac{F_{r, \mathrm{grad}}^{\mathrm{amp}} \sqrt{e} w_{0}}{2 k_{\mathrm{B}} T}-1}=\frac{\pi w_{0}^{2}}{\lambda} \sqrt{\frac{4 n_{\mathrm{m}} a^{3} P}{c k_{\mathrm{B}} T}\left(\frac{m^{2}-1}{m^{2}+2}\right)-1} .
$$

Thus, larger beam waists, larger particle sizes, and higher beam powers ensure longer delivery distances. Let us note that for the beam and particle parameters satisfying inequality (23), the 
maximal longitudinal delivery distance is equal to $2 z_{\mathrm{del}}$, as the particle can be delivered from $-z_{\text {del }}$ to $z_{\text {del }}$. However, if expression (23) does not hold, the maximal longitudinal delivery distance is equal to $z_{\text {del }}+z_{\text {eq }}$, since one can deliver the particle from $-z_{\text {del }}$ to $z_{\text {eq }}$ where the particle will be stopped and stably trapped in 3-D (compare Fig. 3a and Fig. 3b).

The time of the deterministic particle delivery $t_{\text {del }}$ can be easily estimated for an overdamped system, when the inertial term in the equation of motion can be neglected and the particle velocity can be obtained as:

$$
v_{z} \equiv \frac{\mathrm{d} z}{\mathrm{~d} t}=\frac{F_{z}(0, z)}{\gamma}
$$

where $\gamma=6 \pi \eta a$ is the hydrodynamic drag coefficient of the particle and $\eta$ is the viscosity of the surrounding medium. Assuming that the particle is located on the beam axis at all times (i.e., $r=0$ ), an analytical solution of the differential equation (26) can be found; however, the resulting expression is not an easy one to read. Instead, neglecting the contribution of $F_{z, \text { grad }}$ to the net longitudinal force $F_{z}$, one obtains the following solution (assuming that formula (23) is valid) for the delivery time of the particle from $z=0$ to $z=z_{\text {del }}$ :

$$
t_{\mathrm{del}}=z_{\mathrm{del}} \frac{\gamma}{F_{z, \text { scat }}^{\mathrm{amp}}}\left[1+\frac{1}{3}\left(\frac{z_{\mathrm{del}}}{z_{\mathrm{R}}}\right)^{2}\right] .
$$

Obviously, faster delivery over the same distance $z_{\text {del }}$ can be achieved with larger $F_{z \text {,scat }}^{\text {amp }}$ and smaller $\gamma$. For a fixed $z_{\text {del }}$ and beam parameters, the delivery time scales with the particle radius as $a^{-5}$.

Unfortunately, the problem of the delivery time in an underdamped system is more complicated and, generally, an analytical expression for $t_{\text {del }}$ is not accessible. The easiest solution can be obtained if one assumes a constant longitudinal force $F_{z}^{\text {amp }}$. In this case

$$
\frac{\mathrm{d} v_{z}}{\mathrm{~d} t}+\frac{\gamma}{m} v_{z}=\frac{F_{z}^{\mathrm{amp}}}{m}
$$

gives

$$
\begin{aligned}
v_{z}(t) & =\frac{F_{z}^{\mathrm{amp}}}{\gamma}\left(1-\mathrm{e}^{-\frac{\gamma}{m} t}\right)+v_{z}(0) \mathrm{e}^{-\frac{\gamma}{m} t}, \\
z_{\mathrm{del}} & =\int_{0}^{t_{\mathrm{del}}} v_{z}(t) \mathrm{d} t=\frac{F_{z}^{\mathrm{amp}}}{\gamma} t_{\mathrm{del}}+\frac{m}{\gamma}\left(v_{z}(0)-\frac{F_{z}^{\mathrm{amp}}}{\gamma}\right)\left(1-\mathrm{e}^{-\frac{\gamma}{m} t_{\mathrm{del}}}\right) .
\end{aligned}
$$

Assuming the delivery distance $z_{\text {del }}$ is again given by Eq. (25), the delivery time can be obtained by numerically solving the transcendent Eq. (30).

The majority of the experimental demonstrations of object manipulation by Gaussian beams has been done in a liquid medium, using the standard optical tweezers or their holographic variant (see, for example, $[81,136])$. An exciting application of this form of particle delivery is the patterning of glass surfaces with laser-guided metal nanoparticles (also referred to as laser printing [135] - see Fig. 4 for an illustration). Using this technique, it is possible to manufacture plasmonic nanostructures in a fast and simple way (see Fig. 5). The laser wavelength is chosen so that it is close to the plasmon resonance of the metal nanoparticles; thus, the optical pushing force is strongly enhanced [137]. A gold nanoparticle with a diameter of a few tens of nanometers (typically $60-80 \mathrm{~nm}$ ) can be positioned with the accuracy of $50 \mathrm{~nm}$ [135], limited mainly by the residual Brownian motion in the lateral direction. Mutual electrostatic and electromagnetic interactions between adjacent nanoparticles limit their minimal interparticle distance to hundreds of nanometers $[138,139]$. Multiple experimental and theoretical works have considered mutual interactions between individual nanoparticles due to scattered light (so called optical binding) as a 

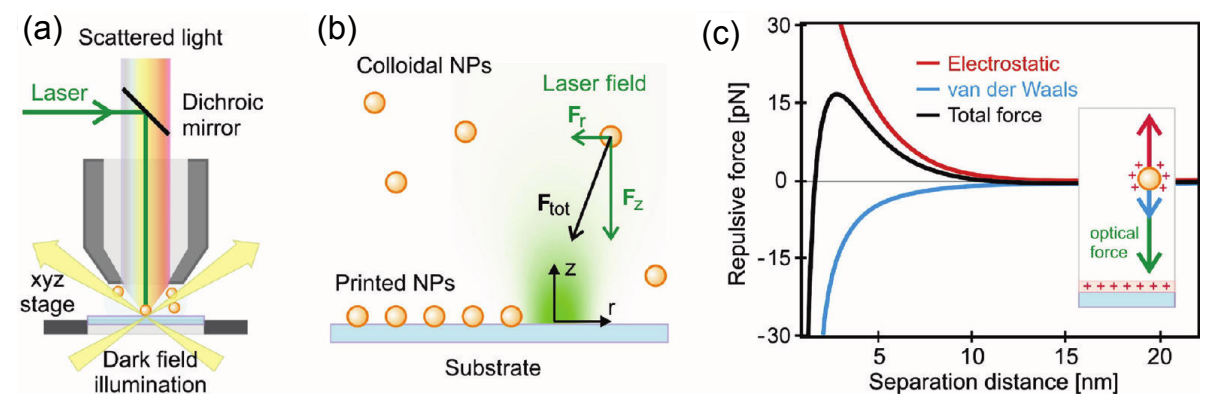

Fig. 4. Employing optical forces for the controlled deposition of nanoparticles (NPs) on solid substrates. (a) Diagram of the experimental setup. A dark-field illumination microscope was adapted to introduce a manipulation laser beam into the optical path via a water immersion objective with a high numerical aperture. This objective was used to simultaneously focus the manipulation beam on the sample and to collect the light scattered by the trapped nanoparticles for imaging purposes. The sample position was controlled with piezo-driven stepper-motor translation stages and the sample was imaged using a digital camera. (b) Schematic representation of the optical forces acting on a NP during the deposition process. (c) Calculated dependence on the separation distance for the electrostatic, van der Waals, and total forces acting on a positively charged Au NP in the vicinity of a positively charged silica surface. Inset: in order to print the NPs, the optical forces need to surpass the net repulsion. Adapted with permission from [135]. Copyright 2010 American Chemical Society.

means for the self-arrangement of nanoparticles [139-145]. By using different laser wavelengths for nanoparticles made of different materials (in particular, $\mathrm{Au}$ and $\mathrm{Ag}$ ) with distinct plasmonic resonances, it was possible to reduce the distance between deposited $\mathrm{Au}$ and $\mathrm{Ag}$ nanoparticles to about $50 \mathrm{~nm}$ [146] (see Fig. 5d-e). This observation indicated that the interaction between the already deposited and incident nanoparticles could include a contribution of thermophoretic origin [147]. Heat dissipation was optimized and Au dimers made of $63 \mathrm{~nm}$ spheres were printed on a reduced graphene oxide layer placed onto a sapphire substrate [146].

Two different laser wavelengths were used simultaneously to orient and deposit Au nanorods with an accuracy of $\pm 16^{\circ}$ [148]. In addition, dual-wavelength illumination was employed to trap plasmonic nanorods [149] or nanoparticles with more complex shapes [150], to align them along the beam polarization direction with a non-resonant laser beam, and to guide them to the surface with a resonant laser beam [151-153]. The heating of highly crystalline gold nanorods excited at their plasmonic resonant frequency was adopted to induce their deterministic bending controllable by the power of the used laser beam. Using optical forces, the resulting V-shaped nanorods were then printed on a supporting substrate [154]. Size-selective optical printing of silicon nanoparticles based on their dipolar magnetic resonances was demonstrated as a very efficient way for surface patterning in liquids [155], which represents a prerequisite for the fabrication of all-dielectric metamaterials or metasurfaces [156-158]. Optically guided plasmonic Janus nanoparticles were positioned on the surface of a cell and, subsequently, injected into the cell by the combined effect of plasmonic heating and optical forces exerted by a focused illumination beam $[159,160]$. Multiplying the number of guiding laser beams using a spatial light modulator (SLM) enabled the implementation of optical force stamping lithography, which provided a faster and more flexible deposition of complex nanoparticle patterns [161] (see Fig. $5 a-b)$.

\subsubsection{Bessel beams}

Lateral and longitudinal variations of the Gaussian beam properties represent their main disadvantage for long-distance particle guiding. A possible solution of this drawback is the use of 

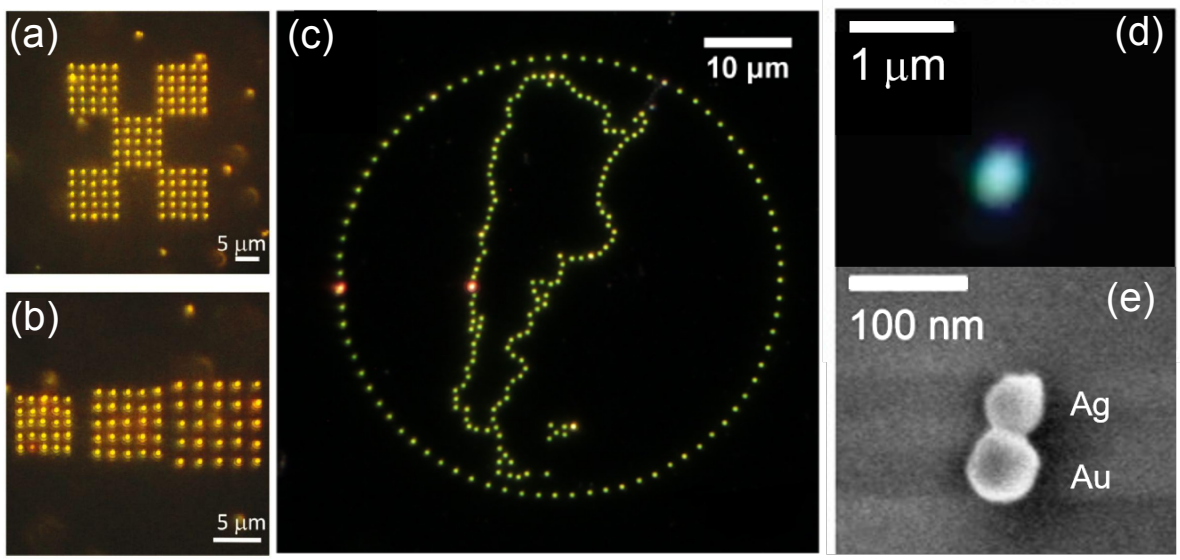

Fig. 5. Deposition of plasmonic nanoparticles on a glass surface. (a) Five square $5 \times 5 \mathrm{Au}$ nanoparticle patterns deposited sequentially by a stamp of $5 \times 5$ Guassian beams [161]. (b) The same method applied to deposit sequentially three 5x5 pattens of Au nanoparticles with different pitch size [161]. (c) A map of Argentina formed by $60 \mathrm{~nm}$ Au nanoparticles [146]. (d) Dark field image of Au and Ag nanoparticles deposited close to each other [146]. (e) The same particle configuration as shown in (d) imaged by scanning electron microscopy. Adapted with permission from [146, 161]. Copyright 2011, 2016 American Chemical Society.

a beam that does not change its lateral and longitudinal properties during propagation and, thus, can guide the particles over longer distances with an (almost) constant velocity. Such beams are called propagation-invariant - or "non-diffracting" - with the paraxial Bessel beam [163-166] being probably the best-known member of this family. Its name comes from the Bessel function of the first kind and $m^{\text {th }}$ order $J_{m}$ that describes the lateral profile of the beam intensity:

$$
E(r, \varphi, z)=E_{0} J_{m}\left(k_{r} r\right) \exp \left(\mathrm{i} k_{z} z\right) \exp ( \pm \mathrm{i} m \varphi)
$$

where $k_{r}=k \sin \beta_{0}$ and $k_{z}=k \cos \beta_{0}$ are the lateral and longitudinal components of the wavenumber $k=\sqrt{k_{r}^{2}+k_{z}^{2}}$, respectively. Paraxial Bessel beams can be viewed as a product of the superposition of an infinite number of plane waves whose wave vectors lie on a conical surface with half-apex angle $\beta_{0}$ (see Fig. 6). The zeroth-order Bessel beam $(m=0)$ is the only Bessel beam having an intensity maximum on the beam axis. In contrast to their mathematical idealization expressed in Eq. (31), real quasi-Bessel beams must possess a finite energy and can only keep the propagation-invariant beam properties over a limited longitudinal distance. They can be experimentally generated using an annular aperture on a SLM [167] or an axicon illuminated with a Gaussian beam $[168,169]$ (see Fig. 6). For the latter method of generating a Bessel beam , the quality of the axicon tip significantly influences the axial properties of the formed Bessel beam [170].

The radius $r_{\mathrm{B}}$ of the central intensity maximum, the axial extent $z_{\mathrm{B}}$ of the ideal zeroth-order 


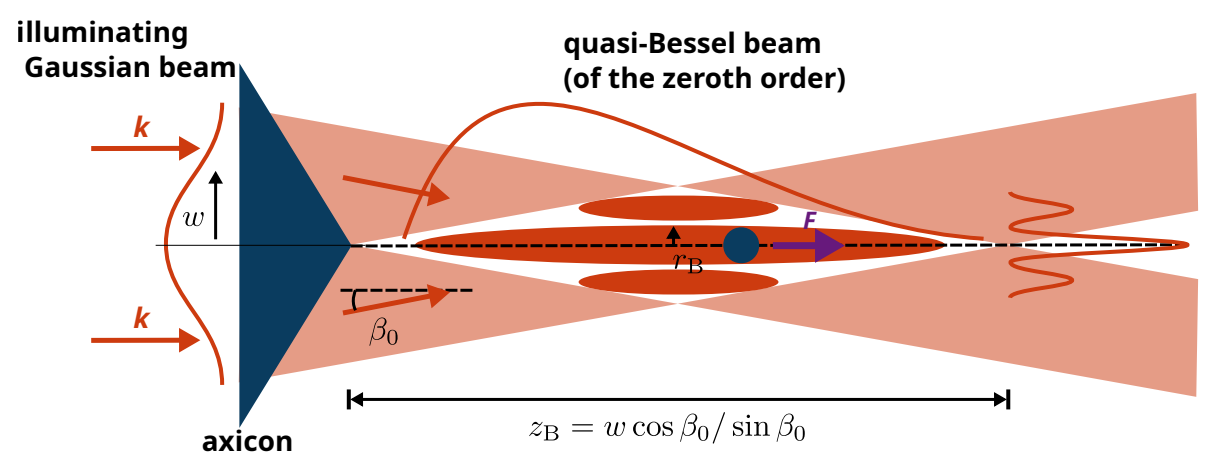

Fig. 6. Schematic representation of a quasi-Bessel beam of zeroth order generated by an axicon illuminated with a Gaussian beam of width $w$ and wavenumber $k$. The quasi-Bessel beam represents a superposition of plane waves with wave vectors lying on a conical surface with half-apex angle $\beta_{0}$. It retains the main characteristics of an ideal Bessel beam: its radial intensity profile is described by the zero-order Bessel function with a constant width of the central core (i.e., the beam is propagation-invariant). However, its on-axis intensity indicated by the solid red curve - is influenced by the profile of the Gaussian beam incident on the axicon. The quasi-Bessel beam exists only over an axial region of length $z_{\mathrm{B}}$ proportional to the value of $w$. Within this region, the beam displays self-healing property: an inserted object with transverse size $d$ disturbs the beam profile significantly only within a conical volume with half-apex angle $\beta_{0}$ and base diameter $d$ [162].

Bessel beam and its optical intensity $I(r)$ are given by [108]

$$
\begin{aligned}
r_{\mathrm{B}} & =\frac{b_{1}}{k \sin \beta_{0}}, \\
z_{\mathrm{B}} & =\frac{r_{\mathrm{A}}}{\tan \beta_{0}}=\frac{r_{\mathrm{A}} k_{z}}{k_{r}} \simeq \frac{r_{\mathrm{A}} k}{k_{r}}, \\
I(r) & =I_{0} J_{0}^{2}\left(k_{r} r\right)=\frac{P_{\mathrm{B}}}{\pi r_{\mathrm{B}}^{2} J_{1}^{2}\left(b_{1}\right)} J_{0}^{2}\left(k_{r} r\right),
\end{aligned}
$$

where $r_{\mathrm{A}}$ denotes the radius of the beam-generating aperture or axicon (for quasi-Bessel beams generated by axicons, $r_{\mathrm{A}}$ is equal to the Gaussian beam half-width $w$ at the axicon), $I_{0}$ is the on-axis optical intensity of the beam, $b_{1} \simeq 2.4048$ corresponds to the first zero crossing of $J_{0}(b)$, and $P_{\mathrm{B}}$ is the power carried by the central core of the Bessel beam. The power carried by each of the concentric rings in the transverse profile of the beam is almost the same as that of the central core. The properties of this Bessel beam can be easily compared with those of a Gaussian beam. Let us assume that the characteristic widths of both beams are related as $r_{\mathrm{B}}=M w_{0}$, where $M>0$ is an arbitrary constant. Assuming that the annular aperture can generate a Bessel beam with $N$ rings, the following simplified relations can be derived [171]:

$$
\begin{aligned}
z_{\mathrm{B}} & \simeq M^{2} N z_{\mathrm{R}} \\
P_{\mathrm{B}} & \simeq \frac{P_{\mathrm{G}}}{N}, \text { assuming } M>1,
\end{aligned}
$$

where $P_{\mathrm{G}}$ is the total power of the Gaussian beam and $z_{\mathrm{R}}$ is its Rayleigh length. Thus, the Bessel beam generally offers a much longer longitudinal delivery distance $z_{\mathrm{B}}$ than the Gaussian beam. A more detailed comparison of delivery distances of the two beams can be obtained using Eq. (25). On the other hand, the power $P_{\mathrm{B}}$ carried by the central core of the zeroth-order Bessel beam, which is available for particle pushing and guiding, is $N$-times lower than the pushing power $P_{\mathrm{G}}$ of a Gaussian beam with the same total power. The seemingly straightforward solution 
of this disadvantage - increasing the total power in the Bessel beam - also leads to an increased light absorption in the surrounding (liquid) medium . At a certain threshold power level, this can eventually induce unwanted fluid convection interfering with the optical guiding of the particles.

The self-healing property of Bessel beams is another of their aspects desirable for optical delivery. If an obstacle of transverse dimension $d$, e.g., a particle of diameter $d$, is placed in the beam, the Bessel beam recovers its transverse intensity profile at distances behind the particle larger than [172]:

$$
z_{\mathrm{rec}}=\frac{d}{2 \tan \left(\beta_{0}\right)}=\frac{d k_{z}}{2 k_{r}} \simeq \frac{d k}{2 k_{r}} .
$$

Intuitively, the illuminated object casts a geometric shadow into the beam, with the axial extent of the shadowed region $z_{\text {rec }}$ given by the diameter of the object and the illuminating angle.

Zeroth-order Bessel beams have been successfully used to deliver multiple microparticles between different sample chambers separated by several millimeters and aligned along the axis of a monochromatic beam [173] (Fig. 7A) or to guide particles over distances of several millimeters using a supercontinuum Bessel beam with a broad spectral bandwidth [174, 175] (Fig. 7B).
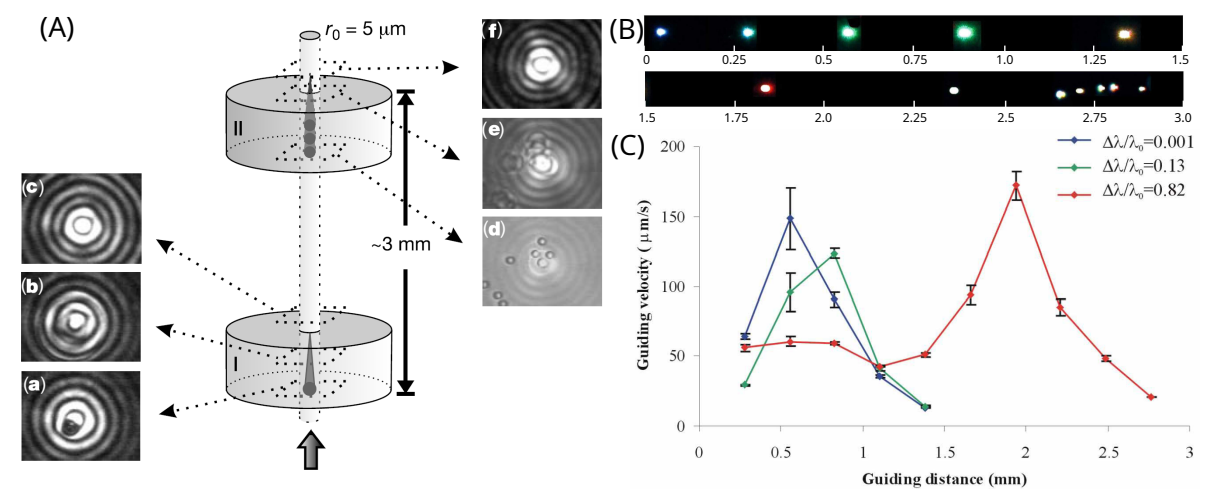

Fig. 7. Manipulation and delivery of microparticles with a single Bessel beam of zeroth order. (A) Simultaneous manipulation of microparticles with different optical properties in two sample chambers (I, II) of depth $100 \mu \mathrm{m}$ and separation distance of $3 \mathrm{~mm}$. Chambers I and II contain aqueous suspensions of hollow and solid silica particles with diameters $5 \mu \mathrm{m}$, respectively. The hollow silica particles with relative refractive index $n_{\mathrm{p}} / n_{\mathrm{m}}<1$ are radially localized in the low-intensity regions, $\mathbf{a}$, whereas the solid particles with relative refractive index $n_{\mathrm{p}} / n_{\mathrm{m}}>1$ localize in the high-intensity regions, $\mathbf{d}$. Immediately after passing a particle, the beam profile is distorted (b,e); however, at the top surface of each chamber, the beam profile is fully recovered (c,f). With a sufficiently high laser power, the particles can be propelled to the top surfaces of their respective chambers. Adapted by permission from Springer Nature Customer Service Centre GmbH: Nature [173] Copyright 2002. (B) Delivery of particles of $5 \mu \mathrm{m}$ diameter over the distance of $3 \mathrm{~mm}$ in a supercontinuum Bessel beam of zeroth order. The light scattered by the particles at different axial locations changes color due to beam dispersion at the axicon. Near the axial distance of $2.75 \mathrm{~mm}$, corresponding to the intensity maximum of infrared wavelengths, convective heating appears and drives the particle away from the beam. (C) Comparison of measured particle guiding velocities and distances in CW (blue), femtosecond (green) and supercontinuum (red) laser beams. The use of supercontinuum Bessel beams enables guiding of particles over the largest distances. Adapted with permission from [176]. Copyright 2005 Optical Society of America.

A higher-order Bessel beam (i.e., one with $|m|>0$ in Eq. (31)) has a helical wavefront. Due to a phase singularity on the beam axis, it forms an intensity minimum along the axis (see Fig. 8); therefore, it is also referred to as doughnut beam or vortex beam. The helicity of the beam, 
characterized by the topological charge $m$, gives rise to an orbital angular momentum equal to $m \hbar$ per photon. The transfer of this angular momentum to a nanoparticle leads to an optical scattering force $F_{\phi}$ in the azimuthal direction. In the Rayleigh approximation, this force can be expressed as:

$$
F_{\phi}= \pm \frac{m}{2} \alpha^{\prime \prime} \varepsilon_{0} \varepsilon_{\mathrm{m}} E_{0}^{2} J_{m}^{2}\left(k_{r} r\right)
$$

where $\varepsilon_{\mathrm{m}}=n_{\mathrm{m}}^{2}$ denotes the relative permittivity of the medium. We refer the reader to Ref. [177] for a more detailed theoretical treatment of the problem.

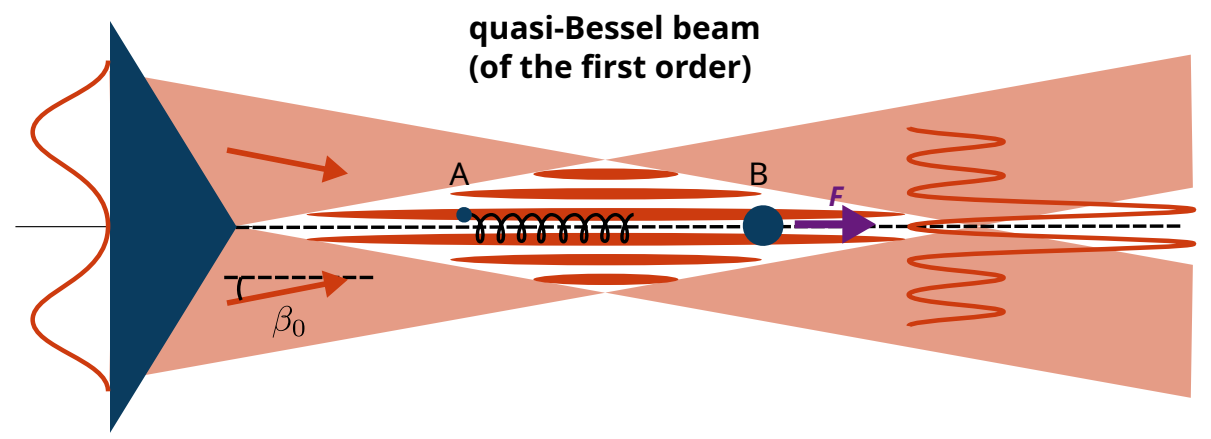

Fig. 8. Schematic representation of a quasi-Bessel beam of first order generated by an axicon illuminated with a Laguerre-Gaussian beam. Similarly to Fig. 6, the generated beam retains the main characteristics of an ideal Bessel beam: its radial intensity profile is described by the first-order Bessel function with a constant width of the central bright ring (i.e., the beam is propagation-invariant). In contrast to the zeroth-order Bessel beam shown in Fig. 6, there is now an intensity minimum on the beam axis. Thus, a small particle (A) with the relative refractive index $n_{\mathrm{p}} / n_{\mathrm{m}}>1$ is confined in the high-intensity ring, with its center localized off the beam axis. Consequently, it is propelled along a spiral path due to the combined effect of the axial and azimuthal scattering forces. On the other hand, the center of a larger particle (B) with the size comparable to the diameter of the central high-intensity ring is localized on the beam axis and the particle moves along a straight path coincident with the beam axis.

If a nanoparticle illuminated by a vortex beam is optically denser than the surrounding medium, it is confined by the gradient force off-axis in the high-intensity part of the beam and, simultaneously, pushed along a spiral trajectory by the sum of the azimuthal and axial scattering forces (see particle A in Fig. 8). However, a larger particle that laterally overlaps with one or more high-intensity rings can localize its center on the beam axis $[178,179]$, and, subsequently, it is pushed straight along the axis (see particle B in Fig. 8). A particle with a refractive index lower than that of the surrounding medium (e.g., a hollow glass microsphere immersed in water) is repelled from the high-intensity region of the beam. Thus, upon illumination with a vortex beam featuring an intensity minimum on its axis, the particle can be laterally confined there $[3,180]$. In order to keep such a low-index particle confined on the beam axis, the dimensions of the particle should be smaller than the diameter of the central high-intensity ring of the beam [181]. In this case, the edges of the particle overlap with the high-intensity ring of the beam and the particle is again pushed along the beam propagation direction by optical forces. However, if the particle absorbs the light of the guiding beam, thermal forces could dominate over radiation pressure and different underlying physics must be considered (see Section 5).

A similar mechanism has been also proposed for delivering cold atoms confined in the high-intensity or low-intensity regions of vortex beams that were red-detuned or blue-detuned relatively to the atomic transition frequency, respectively [182,183]. 


\subsection{3. "Curved" beams}

(a) "curved" Bessel beam

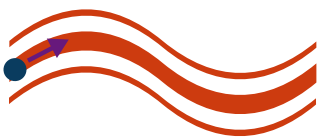

(b) "solenoidal" beam

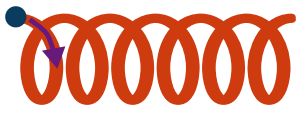

(c) "accelerating" beam

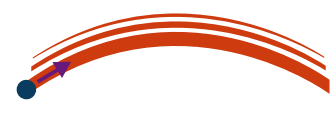

Fig. 9. Examples of optical beams that propagate along curved trajectories in homogeneous media and have been used for optical guiding of objects laterally confined in the beam due to the gradient optical force. (a) A "curved" Bessel beam [167, 184-186], (b) a "solenoidal" beam forming a high-intensity spiral, along which the energy propagates through the medium [187, 188], (c) an "accelerating" beam bending along its propagation axis with a smaller bending radius compared to the case shown in (a). Various types of accelerating beams exist that are usually named after the functions or differential equations describing their properties, e.g., Airy [189, 190], Mathieu [191, 192] or parabolic (Weber) beams [193].

The utilization of diffractive optical elements, such as holograms and phase gratings, offers the possibility of shaping the Bessel beam intensity profile not only laterally [194], but also along the beam axis [167]. It is even possible to generate beams that do not propagate along a straight line in a homogeneous medium, but instead follow a curved "snaking" or spiraling trajectory [184-186] (see Fig. 9a). Using such curved beams, particles can be guided around non-transparent obstacles, which has interesting applications in microfabrication or particle sorting.

Optical guiding of particles along helical trajectories has been also demonstrated using an optical solenoid beam [187,188], a complicated beam shape that represents an example of a rotating scale-invariant field or a beam with twisted phase and amplitude [188] (see Fig. 9b). Practically, solenoid beams can be formed by interference of several co-propagating optical vortex beams with various topological charges $m$. Their electric field within the longitudinal region of their existence can be described as:

$$
E_{\gamma, \ell}(r, \theta, z)=A \sum_{m=[\ell-\gamma k]}^{[\ell]} \frac{\ell-m}{\gamma^{2}} J_{m}\left(q_{m} R\right) e^{i \frac{\ell-m}{\gamma} z} e^{i m \theta} J_{m}\left(q_{m} r\right),
$$

where $A$ is an amplitude, $\gamma / \ell$ and $\gamma$ denote the mutually independent pitches of the phase and intensity helix, respectively, $[x]$ denotes the integer part of $x, R$ is the radius of the intensity spiral and $q_{m}^{2}=k^{2}-(\ell-m)^{2} / \gamma^{2}$, with $k$ being the wavenumber of the source optical wave used to generate the solenoid beam. Intensity maxima of solenoid beams trace curves in three dimensions, with arbitrary amplitude and phase profiles along these curves. Examples of the intensity spirals with different wavefronts are shown in Fig. 10. A particle with the refractive index higher than the surrounding medium is confined to the high-intensity spiral by the gradient force and - according to the local orientation of the beam wavevector that is perpendicular to the wavefronts at each location - it is pushed along the intensity spiral in the forward direction (Fig. 10a) or in the backward direction (Fig. 10c).

There exist other types of curved beams (also referred to as accelerating) suitable for optical manipulation, which do not change their lateral intensity profile over certain propagation length (i.e., they are propagation-invariant; see Fig. 9c). Such beams also display self-healing properties, i.e., they recover their lateral intensity profiles at a certain propagation distance behind an obstacle. For example, exponentially apertured Airy beams $[189,190]$ have been generated experimentally using cubic phase masks imposed on an SLM [195-197]. With Airy beams, particles can be delivered along parabolic trajectories [198], including transport between two compartments separated by a vertical wall [199] (see Fig. 11). SLMs also enable the generation of arrays of 

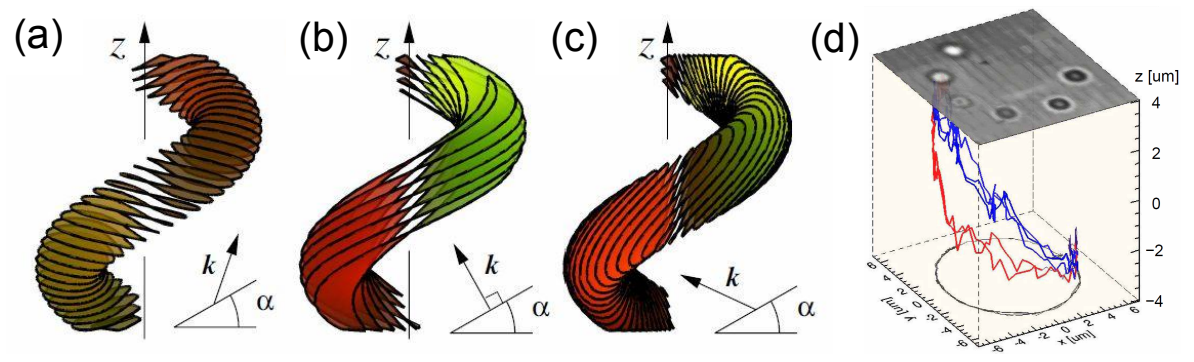

Fig. 10. Optical guiding of particles with solenoid beams. The direction of particle motion along the high-intensity helix propagating in the positive $z$-direction depends on the actual orientation of the wavevector $\mathbf{k}$ in the solenoid beam for given values of $\ell$ and $m$ (see Eq. (39)). (a) When $\ell>0$, the projection of $\mathbf{k}$ on the helix points forward along the local helix direction and the particle moves along the positive direction of the $z$-axis. (b) When $\ell=0$, the beam wavefronts are parallel to the helix. Since $\mathbf{k}$ is everywhere perpendicular to the local direction of the helix, the particle feels no net pushing force along the beam. (c) When $\ell<0$, the projection of $\mathbf{k}$ on the helix points backward along the local helix direction and the particle moves along the negative direction of the $z$-axis. The pitch of the helix was fixed with $\alpha=\arctan (\gamma k)$ for all three cases (a) - (c). (d) Three-dimensional trajectories of silica spheres dispersed in water, moving along one turn of the helix along the positive $z$-axis ( $l=30$, blue curves) and along the negative $z$-axis $(l=-30$, red curves). The top image shows a single particle at 6 consecutive positions along its trajectory. Adapted with permission from [187]. Copyright 2010 Optical Society of America.

multiple Airy beams [200,201]; such configurations have been tested, for example, for optical path clearing in a turbid medium formed by a dense colloidal suspension.

The so-called autofocusing circular Airy beams represent radially symmetric light beams that focus laterally (either inward or outward) during propagation, thus displaying transverse acceleration [202]. In the far-field, these beams morph into non-diffracting Bessel beams. Inward-focusing Airy beams have been successfully used for particle transport, serving as virtual tapered channel guides for microparticles [202] (see Fig. 12).

The paraxial accelerating Airy beams discussed above can bend only slightly along their propagation direction before breaking the validity of the paraxial approximation. A substantial advance to larger bending angles of almost $180^{\circ}$ came with the solutions of the full vectorial Maxwell's equations [203]. Since these equations have a complete symmetry with respect to the lateral $x, y$ and longitudinal $z$ directions, a solution in the form of a circular trajectory can be found. Non-paraxial shape-preserving parabolic [204], Weber [193], Mathieu [191, 192, 205], or arbitrary transverse profile [206,207] beams have been reported theoretically. The only experimental demonstration of the use of non-paraxial beams for particle transport reported so far used half-Bessel beams to guide particles in absorbing media without suffering an intensity decay along the beam propagation path [208] (see Fig. 13). 
(a)

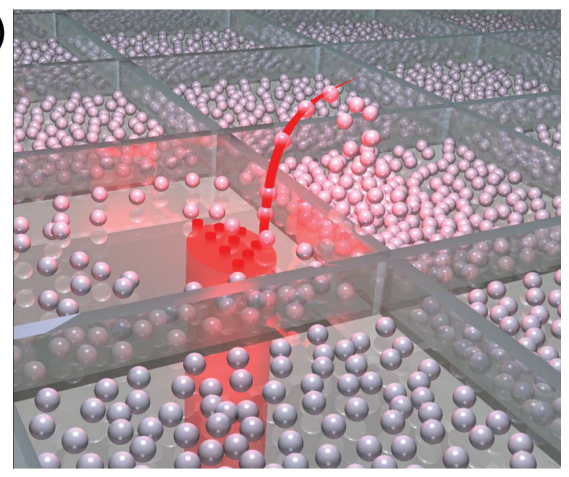

(b)

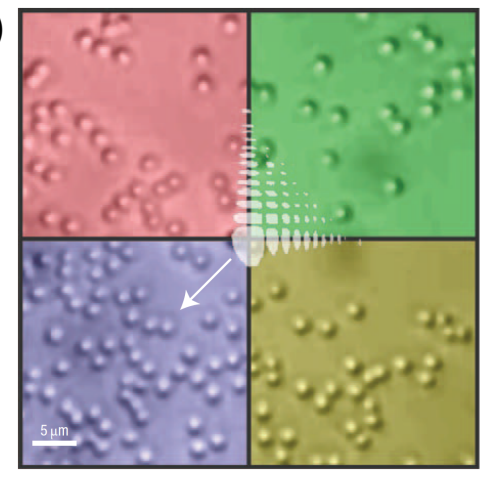

Fig. 11. Optical guiding of particles with accelerating Airy beams. (a) Illustration of the transport of particles over a solid wall. Adapted with permission of Royal Society of Chemistry, from [199]. Copyright 2009; permission conveyed through Copyright Clearance Center, Inc. (b) Transport of suspended particles between different compartments within the sample chamber. Illumination of the particle suspension with an Airy beam, whose lateral cross-section is denoted with the white pattern, induces particle motion along the white arrow from the green to the purple compartment. Adapted by permission from Springer Nature Customer Service Centre GmbH: Nature Photonics [198] Copyright 2008.

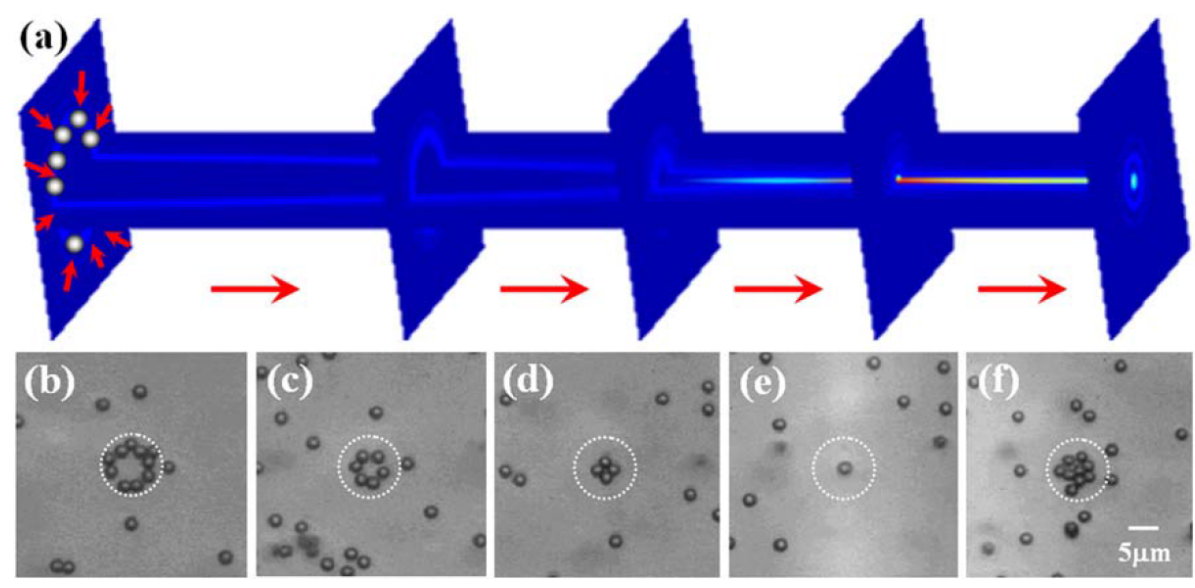

Fig. 12. Demonstration of particle transport in a radially symmetric autofocusing Airy beam. (a) Illustration of particles' motion through a virtual tapered particle guide formed by an inward-focusing Airy beam. The red arrows indicate the direction of motion of the particles (white circles) and the color map describes the intensity profile of the autofocusing beam. (b)-(f) Snapshots of guided $2 \mu \mathrm{m}$ polystyrene particles acquired at different lateral planes along the beam axis whose positions are indicated in (a). Initially, the particles are trapped within the high-intensity ring of the beam (b). As the beam gradually contracts during the propagation, the particles are pushed towards its axis (c), (d). After passing the focal point (e), the beam morphs into a zero-order Bessel beam (f). Dashed circles serve as a reference for the initial diameter of the beam. Adapted with permission from [202]. Copyright 2011 Optical Society of America. 
(a)

(b)

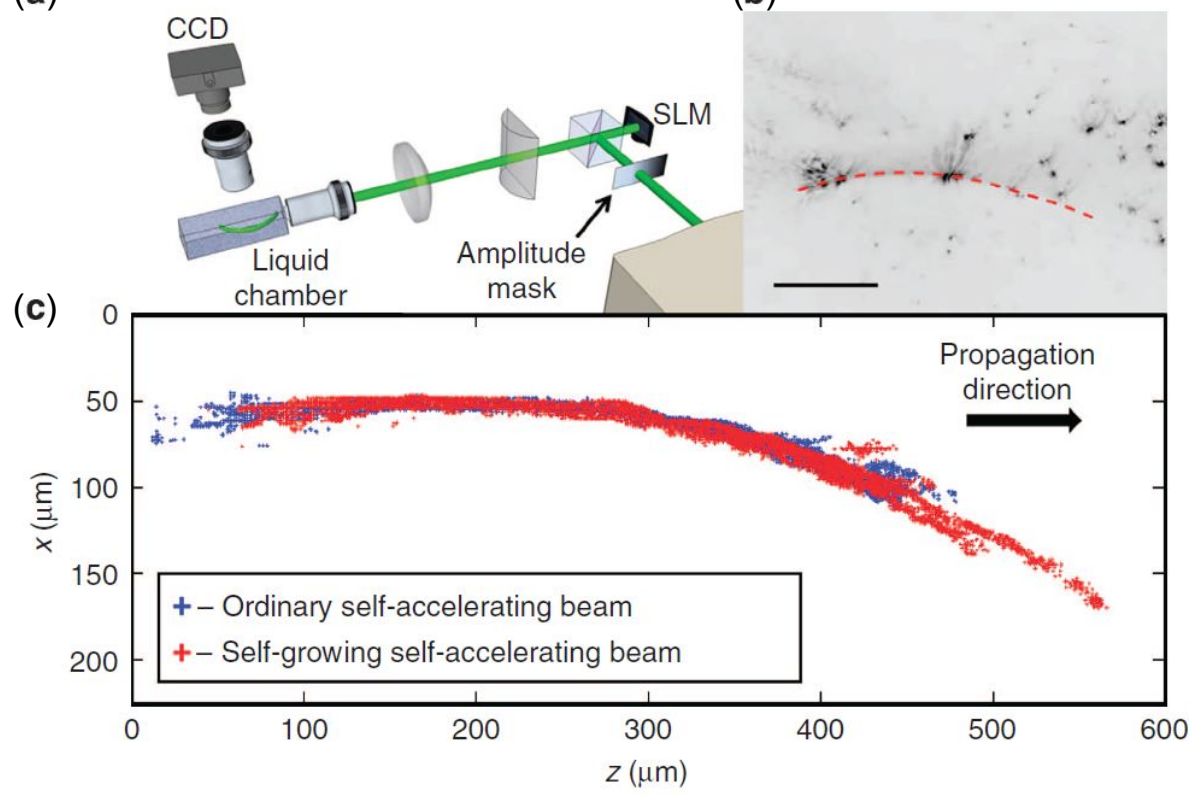

Fig. 13. Dynamics of microparticles transported along a circular trajectory by a non-paraxial accelerating beam. (a) Experimental setup. The phase and amplitude of an incident broad Gaussian beam are modulated using a SLM and an amplitude mask to produce an accelerating beam that is subsequently directed into a liquid sample chamber. (b) A typical image of a guided polystyrene particle of diameter $10 \mu \mathrm{m}$ with its trajectory in the beam highlighted by the red dashed curve. The scale bar corresponds to $250 \mu \mathrm{m}$. (c) Experimentally observed particle trajectories in an ordinary non-paraxial self-accelerating beam (blue) and a beam with exponentially growing amplitude compensating for losses in the medium (red). The compensated beam enables guiding of the particles along longer curved trajectories. Adapted by permission from Springer Nature Customer Service Centre GmbH: Nature Communications [208], Copyright 2014. 


\subsection{3-D optical pulling in tractor beams}

The pulling force is understood as an optical force that has a component pointing against the direction of the incident field wavevector. Usually, it comes from the photon recoil in carefully designed beam geometries. Recently, several beam configurations providing such optical pulling force have been introduced; they are collectively referred to as optical tractor beams $[13,71,110,111,209-212]$. Figure 14 illustrates how the pulling force along the horizontal axis results from the deflection of the scattered photons towards this axis. Since the horizontal component of the photon momentum increases upon scattering, the conservation of the total momentum of the system particle + light requires the particle to move in the opposite direction. Such a scenario arises, for example, when a converging (diverging) beam is collimated by a negative (positive) lens: the lens would be pulled against the beam propagation if it were not fixed. To maximize this effect, Lundenberg lenses characterized by a radial gradient of refractive index were proposed [213]. The same phenomenon is also present in optical tweezers when a spherical particle with the relative refractive index $m>1$, acting as a positive lens, is placed behind the beam focus and collimates the beam that would otherwise diverge there. However, a net pulling force only exists over a limited range of longitudinal distances of the particle from the focus, within which the force contribution from the rays that are bent by the particle towards the beam axis dominates over the forward radiation pressure. Within the ray optics regime, the mechanism described above explains the origin of the final axial optical force resulting from the interplay of the gradient force pointing towards the beam focus and the radiation pressure force pointing along the beam propagation direction.
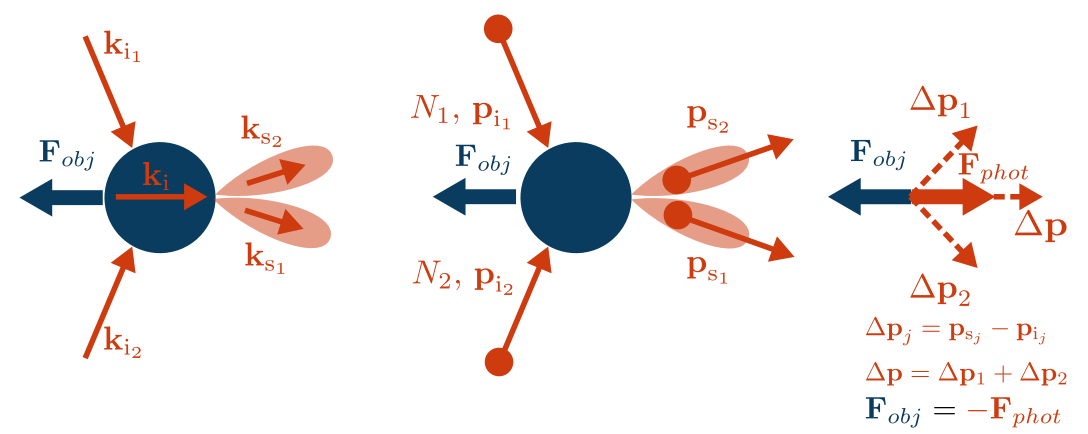

Fig. 14. An elementary explanation of the origin of the optical pulling force $\mathbf{F}_{\text {obj }}$. This force arises due to the particular way in which photon trajectories deflect upon scattering from a particle. For the sake of clarity, only two representative trajectories of the corresponding incident and scattered photons are shown, characterized by the incident and scattered wavevectors $\mathbf{k}_{\mathbf{i}_{1}}, \mathbf{k}_{\mathbf{i}_{2}}$ and $\mathbf{k}_{\mathrm{s}_{1}}, \mathbf{k}_{\mathrm{s}_{2}}$, respectively. The corresponding incident and scattered linear momenta associated with these photons are then $\mathbf{p}_{\mathrm{i}_{1}}, \mathbf{p}_{\mathrm{i}_{2}}$ and $\mathbf{p}_{\mathrm{s}_{1}}, \mathbf{p}_{\mathrm{s}_{2}}$, and their momentum change upon scattering can be expressed as $\Delta \mathbf{p}_{j}=\mathbf{p}_{\mathrm{s}_{j}}-\mathbf{p}_{\mathrm{i}_{j}}$, with $j=1,2$. Assuming that $N_{1}$ and $N_{2}$ photons are respectively incident along the two trajectories in a time interval $\Delta t$, one can use Newton's second law to express the net force $\mathbf{F}_{\text {phot }, j}$ acting on the $N_{j}$ photons as $\mathbf{F}_{\text {phot }, j}=N_{j} \Delta \mathbf{p}_{j} / \Delta t$. According to Newton's third law, the force exerted on the particle by photons scattered along the $j$-th trajectory is $\mathbf{F}_{\mathrm{obj}, j}=-\mathbf{F}_{\text {phot } j}$. The total force $\mathbf{F}_{\text {obj }}$ exerted on the particle is then equal to the vector sum of the forces

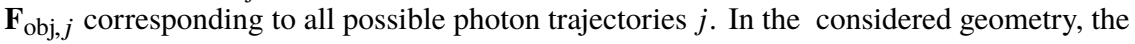
horizontal component of the total force $\mathbf{F}_{\text {obj }}$ is oriented against the direction of the net horizontal component of the incident wavevector. The particle is thus pulled against the direction of propagation of the incident beam.

Figure 15 shows the simplest geometry that was used for the experimental demonstration of optical pulling forces [13]. This configuration uses two wide Gaussian beams incident upon 

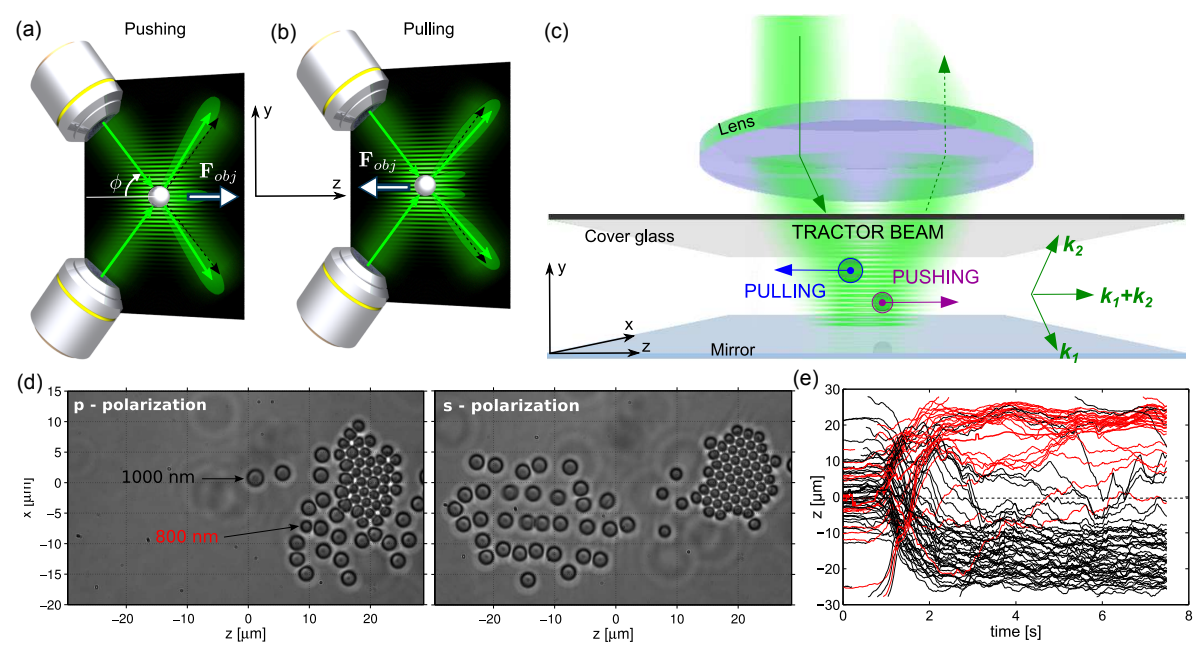

Fig. 15. Simplified scheme of the experimental demonstration of optical pulling forces. Two wide interfering Gaussian beams create intensity fringes that localize the particle in the $y$-direction. Depending on the angle of incidence $\phi$, particle size and polarization of the incident beams, the photons are predominantly scattered either off (a) or towards (b) the horizontal $z$-axis, resulting in a pushing or a pulling force $\mathbf{F}_{\text {obj }}$ along the $z$-axis, respectively. The black dashed arrows guide the eye in the direction of the beams incident on the particle. The green solid arrows indicate the predominant directions of the incident and scattered photons. (c) A Gaussian beam incident off-axis on a positive lens is focused on a dielectric mirror and retro-reflected backwards. Reflection of the downward-propagating beam from the horizontal mirror provides the upward-propagating beam shown in (a) and (b) and makes the experimental setup easier to align while keeping the essential mechanism the same. Vectors $\mathbf{k}_{1}, \mathbf{k}_{2}$, and $\mathbf{k}_{1}+\mathbf{k}_{2}$ indicate the directions of the incident, reflected and overall wavevector, respectively. (d) An example of optical sorting of polystyrene spheres of diameter $800 \mathrm{~nm}$ and $1 \mu \mathrm{m}$ by switching the polarization of the tractor beam shown in (c) from "p", pushing all particles to the right, to "s", pulling the larger spheres to the left instead. (e) Trajectories of individual particles from (d) during the sorting, with the red ones corresponding to the smaller particles. Adapted by permission from Springer Nature Customer Service Centre GmbH: Nature Photonics [13], Copyright 2013.

an object at large angles with respect to the pulling/pushing axis (directed horizontally in the figure). If the two beams interfere, a standing wave is created and particles can be confined at different nodes (or antinodes) of the resulting interference structure. In order to simplify the alignment, two independent beams were replaced with a combination of a single beam incident nearly perpendicularly upon a dielectric mirror and a beam retro-reflected from the mirror. The nodes and antinodes of the formed standing wave were then parallel to the surface of the mirror (see Fig. 15c). The experiments reported in [13] demonstrated that the occurrence of particle pushing or pulling forces depends on the particle size and the particle's behavior can be tuned by changing the angle of incidence $\phi$ of the beam on the mirror . In addition, the pushing or pulling motion can be induced by switching between the p- and s-polarization of the incident beam, respectively. As a result, under proper illumination, a mixture of different-size particles could be spatially sorted, as these particles were pulled or pushed in opposite directions [13] (see Fig. 15d-e). When multiple particles are illuminated simultaneously, they can interact via the scattered light, in a process referred to as optical binding $[140,141,214]$. The particles organized in optically bound structures feel stronger pulling forces; thus, they move more rapidly and their direction of motion is opposite to that of individual particles (see Fig. 16a-c). Therefore, this 
arrangement seems to be well suited for delivering simultaneously multiple particles in the form of optically bound non-spherical structures [13]. Several theoretical studies have also considered the effect of multiple particles on the tractor beam performance [215,216], typically showing an enhancement of the pulling effect.
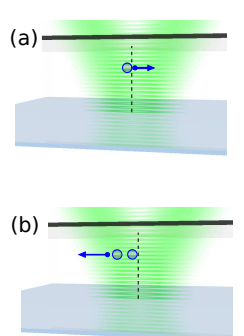

(c)

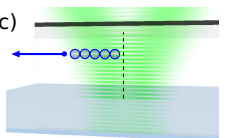

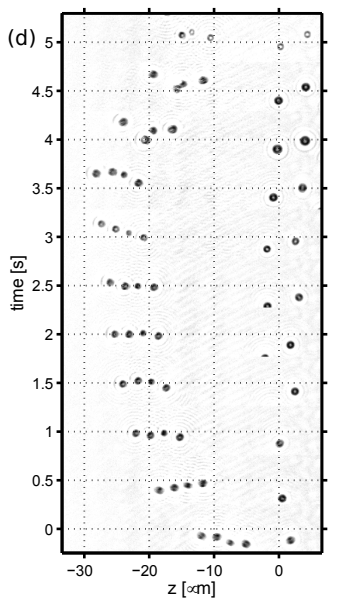
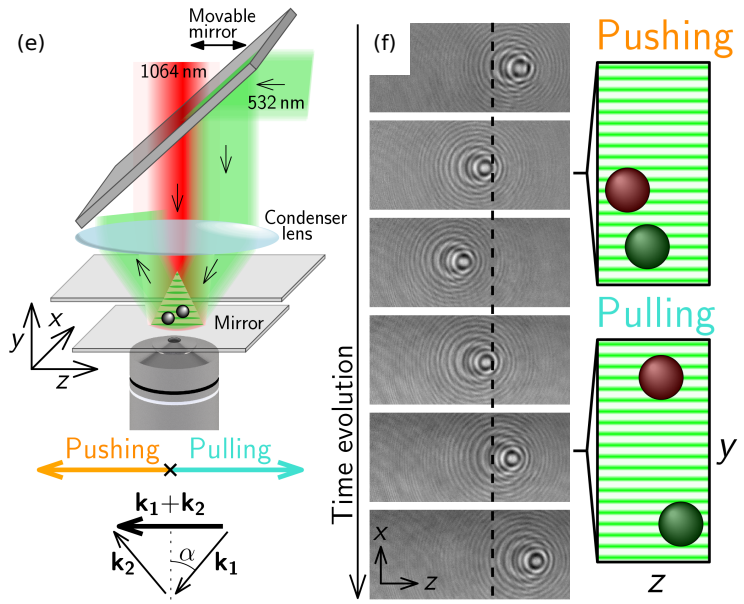

Fig. 16. Demonstration of enhanced optical pulling of an optically bound structure. (a) An individual particle is pushed to the right, while (b) an optically bound structure is pulled to the left. (c) The net force increases with increasing number of optically bound particles in the structure. (d) Time series of snapshots from an experiment. An illuminated structure formed by four optically bound particles displays net motion of its center of mass, in contrast to a single particle that remains stationary during the experiment. Adapted by permission from Springer Nature Customer Service Centre GmbH: Nature Photonics [13], Copyright 2013. (e) Geometry of a similar guiding experiment with optically bound spheres located at different interference fringes. Note that the pushing and pulling directions are reversed with respect to the previously discussed cases. (f) Holograms of two optically bound spheres at different time instants. As illustrated by the schematics shown on the right, the direction of particles' motion (pushing or pulling) depends on the vertical inter-particle distance along the $y$-axis. Adapted under Creative Commons Attribution 4.0 International License from [216].

The wavevectors of the two wide Gaussian beams forming the tractor beam shown in Fig. $15 a-b$ lie on the opposite sides of the surface of a cone with half-apex angle $\phi$. Hence, these beams can be viewed as two particular components of an infinite spectrum of plane waves that can generate a zeroth-order Bessel beam upon interference [163, 164] (see also Fig. 6). The similarity between the two field configurations implies the possibility of using Bessel beams for optical pulling. Indeed, calculations suggest that if the half-apex angle $\phi$ is larger than about $\pi / 3$, a high-index spherical particle can be laterally confined in the high-intensity core of the Bessel beam and, simultaneously, can be pulled against the direction of the beam propagation [71, 110,112,217-219]. Unfortunately, an experimental demonstration of this concept is still missing and delivery of spherical particles over longitudinal distances on the order of tens of micrometers seems to be unrealistic, because a large refractive or reflective conical surface would be needed to produce the Bessel beam with the required parameters. A more optimistic outlook for practical applications of this idea was presented in a recent theoretical study suggesting that a cylindrical object with anti-reflection coated facets can feel a pulling force for $\phi$ as low as $1^{\circ}$ over longitudinal distances on the order of $14 \mathrm{~cm}$ [220]. In this context, it is worth mentioning that pulling forces have been also observed with acoustic propagation-invariant beams [221-224] and discussed in connection with matter waves [225]. 
Beyond the induced electric dipole $\mathbf{d}$, the simplest theoretical description of the pulling force has to include also the induced magnetic dipole $\mathbf{m}$. If both induced dipoles are expressed using the Mie scattering coefficients $a_{1}$ and $b_{1}$ [117], the validity of this theoretical description extends well beyond the limits of the Rayleigh approximation [226, 227]:

$$
\begin{aligned}
& \mathbf{d}=\varepsilon_{0} \varepsilon_{\mathrm{m}} \alpha_{\mathrm{e}} \mathbf{E} \text {, where } \alpha_{\mathrm{e}}=\mathrm{i} \frac{6 \pi a_{1}}{k^{3}}, \\
& \mathbf{m}=\alpha_{\mathrm{m}} \mathbf{H}, \text { where } \alpha_{\mathrm{m}}=\mathrm{i} \frac{6 \pi b_{1}}{k^{3}} .
\end{aligned}
$$

The time-averaged force can then be expressed as $[155,226,228]$ :

$$
\begin{aligned}
\mathbf{F} & =\frac{1}{2} \mathfrak{R}\left\{\sum_{i=x, y, z} d_{i} \nabla E_{i}^{*}\right\}+\frac{1}{2} \mathfrak{R}\left\{\sum_{i=x, y, z} m_{i} \nabla B_{i}^{*}\right\}-\sqrt{\frac{\mu_{0}}{\varepsilon_{0} \varepsilon_{\mathrm{m}}}} \frac{k^{4}}{12 \pi} \mathfrak{R}\left\{\mathbf{d} \times \mathbf{m}^{*}\right\}, \\
& =\mathbf{F}_{\mathrm{e}}+\mathbf{F}_{\mathrm{m}}+\mathbf{F}_{\mathrm{e}-\mathrm{m}},
\end{aligned}
$$

where the first two terms come from the induced electric and magnetic dipoles, respectively, and the third term is the result of the interference between the fields radiated by the two dipoles. In Eq. (42), $d_{i}\left(m_{i}\right)$ are the components of the induced electric (magnetic) dipole $\mathbf{d}(\mathbf{m})$, respectively. The interference of the fields radiated by $\mathbf{d}$ and $\mathbf{m}$ leads to an asymmetry between the forward and backward scattering and gives rise to the pulling force effect. The term $\mathbf{F}_{e}$ in Eq. (43) is the electric force acting on a Rayleigh nanoparticle, which can be rewritten in the form of Eq. (10). A similar procedure can be applied to the magnetic force $\mathbf{F}_{m}$ using the corresponding quantities for the magnetic field. However, the nature of the force component $\mathbf{F}_{e-m}$ is different; this term can be rewritten as [226]:

$$
\mathbf{F}_{\mathrm{e}-\mathrm{m}}=-\sqrt{\frac{\mu_{0}}{\varepsilon_{0} \varepsilon_{\mathrm{m}}}} \frac{k^{4}}{12 \pi} \mathfrak{R}\left\{\alpha_{\mathrm{e}} \alpha_{\mathrm{m}}^{*}\right\} \mathfrak{R}\left\{\mathbf{E} \times \mathbf{H}^{*}\right\}+\frac{\mu_{0} k^{3}}{12 \pi} \mathfrak{J}\left\{\alpha_{\mathrm{e}} \alpha_{\mathrm{m}}^{*}\right\}\left\{\frac{1}{2} \nabla|\mathbf{E}|^{2}-\mathfrak{R}\left\{\left(\mathbf{E}^{*} \cdot \nabla\right) \mathbf{E}\right\},\right.
$$

where $\varepsilon=\varepsilon_{0} \varepsilon_{\mathrm{m}}$ and $\mu_{0}$ denote the permittivity and permeability of the medium, respectively, and the medium is assumed to be non-magnetic, i.e., $\mu=\mu_{0}$. If the field gradients in the second term of Eq. (44) are negligible, the force $\mathbf{F}_{\mathrm{e}-\mathrm{m}}$ is negative and acts against the radiation pressure for $\mathfrak{R}\left\{\alpha_{\mathrm{e}} \alpha_{\mathrm{m}}^{*}\right\}>0$, when the electric and magnetic dipole emit radiation in phase and scatter strongly in the forward direction. This effect is strongest under the first Kerker condition $\varepsilon=\mu$ [227] when the backward scattering is zero. In contrast, the minimal (nonzero) forward scattering is obtained under the second Kerker condition $\varepsilon=-(\mu-4) /(2 \mu+1)$ when the electric and magnetic dipoles oscillate $180^{\circ}$ out of phase. A more accurate analytical expression for the force terms in Eq. (42) can be obtained using higher order multimodes in the expansion [110].

A different scheme for obtaining an optical pulling force was proposed for manipulating atoms that were simultaneously pumped by two counter-propagating interfering beams forming a standing wave and by another independent laser beam perpendicular to the two interfering beams [229,230] (see Fig. 17 for an illustration of the experimental geometry). This mechanism was based on the pulling recoil generated by a photon emitted by stimulated emission in the direction of the independent pumping beam.

As suggested by the experiments with optically bound structures described in Fig. 16d, stronger optical pulling forces can be produced when objects lacking full spherical symmetry are considered. To this end, several theoretical studies have predicted the possibility of obtaining an optical tractor beam for cylinders [231], lossy nanoparticles made of hyperbolic metamaterials [232], and chiral particles [233].

\subsection{Counter-propagating beams and optical conveyor belts}

In the context of optical trapping, an experimental geometry with two counter-propagating beams was first used by Arthur Ashkin in his pioneering demonstration of the full three-dimensional 


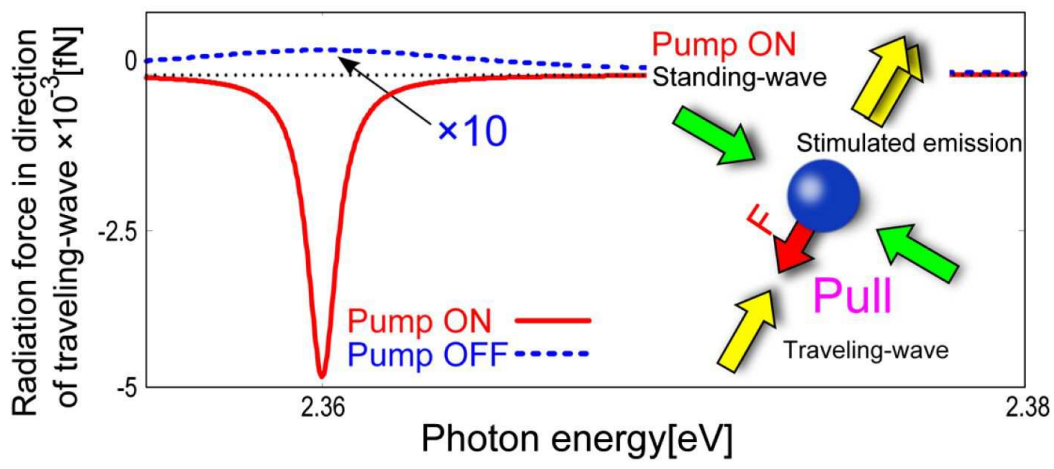

Fig. 17. The use of stimulated emission for generating optical pulling forces on atoms. Right: a traveling wave (yellow) initiates stimulated emission in the atom (blue) that is optically pumped by two counter-propagating beams forming a standing wave (green). A photon emitted by stimulated emission kicks the atom backwards (red), against the direction of the incoming traveling wave. Left: comparison of optical forces acting on the atoms due to the traveling wave in the presence (red) and absence (blue) of optical pumping by the standing wave. Upon turning the standing wave on, the optical force becomes negative and its magnitude significantly increases. Adapted with permission from [229]. Adapted with permission from [229]. Copyright 2012 by the American Physical Society.

all-optical trapping of latex microparticles [1]. Since then, this geometry has been referred to as the dual-beam trap.

When dealing with optical manipulation based on two counter-propagating laser beams, two possible scenarios can arise. In the first one, the two trapping beams do not interfere with each other due to their mutual incoherence or perpendicular polarization. Because of the relaxed requirements on the phase and polarization relationship between the two beams, this dual-beam geometry is rather flexible and can be implemented in various configurations based on bulk optics or optical fibers, schematically illustrated in Fig. 18. This geometry will be analyzed in Section 3.3.1. Alternatively, the two trapping beams can interfere, producing a standing wave with a periodic structure of intensity maxima and minima. This structure can subsequently function as a microscopic conveyor belt for simultaneous delivery of multiple particles. Optical conveyor belts will be discussed in detail in Section 3.3.2.

\subsubsection{Dual-beam geometry}

Figure 18a-b illustrates the classical free-space dual-beam geometry with the position of the dual-beam trap in the sample cell. The trap corresponds to the stable equilibrium position where a particle is confined if it is optically denser than the surrounding medium. When both beams have exactly the same properties, symmetry considerations dictate that the particle is trapped half-way between the beam focal planes. However, if the intensity of beam 1 is higher, the particle is pushed toward the focal plane of the lower intensity beam 2, until the stronger scattering force of beam 1 is compensated by the scattering force of beam 2 and by the gradient forces of both beams due to intensity gradients in their axial direction. Adjustment of the relative power of the two beams can then lead to controlled bidirectional guiding of the particle. The whole experimental setup and its alignment can be significantly simplified if the two free-space propagating laser beams are replaced with optical fibers facing each other. This arrangement is known as a fiber trap. Figure 18c-f shows a schematics of the experimental setup with details of the trapping region for three different configurations of fibers. In addition to single-mode fibers [234], multimode fibers [235-237], photonic crystal fibers [238] and surface waveguides [239, 240] have been also 
(a)

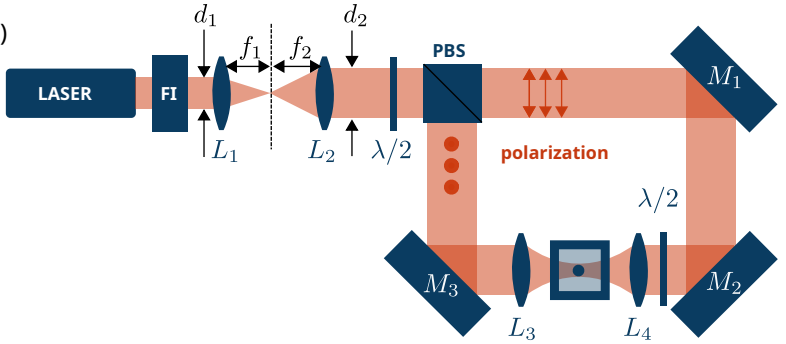

(b)

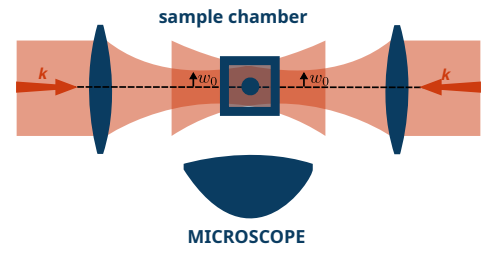

(c) (d)

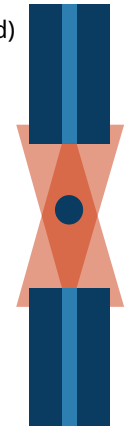

(e)

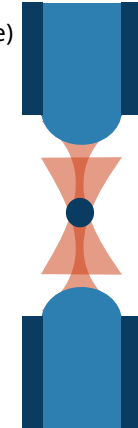

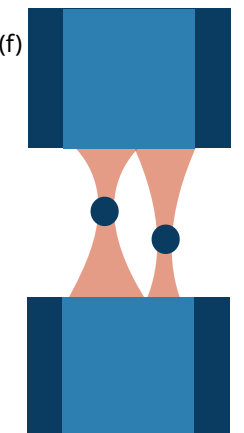

Fig. 18. Different implementations of a dual-beam setup for optical manipulation. (a) A laser beam passes through a Faraday isolator (FI), blocking the light back-reflected toward the laser, and through lenses $L_{1}$ and $L_{2}$ with focal lengths $f_{1}$ and $f_{2}$, respectively, that magnify the original beam diameter $d_{1}$ to $d_{2}$ fulfilling $d_{2}=d_{1} f_{2} / f_{1}$. The final diameter $d_{2}$ should slightly overfill the input aperture of the focusing lenses $L_{3}$ and $L_{4}$. A $\lambda / 2$-wave plate controls the intensity balance between the two beams obtained from the original one after a polarizing beam splitter (PBS). The two beams leaving the PBS have perpendicular polarizations; they follow their respective optical paths, reflect at mirrors $M_{1}-M_{3}$ and are finally focused into the sample chamber by lenses $L_{3}$ and $L_{4}$ with identical focal lengths, $f_{3}=f_{4}$. The diameter $D$ of the beam focus can be estimated from the Airy disc diameter as $D \simeq 1.22 \lambda_{0} / \mathrm{NA}$, where $\lambda_{0}$ and NA denote the vacuum wavelength and the numerical aperture of lenses $L_{3,4}$, respectively. (b) The sample chamber is observed perpendicularly to the propagation direction of the manipulation beams, using a standard optical microscope equipped with a long-working distance objective. (c) An alternative setup using a laser beam coupled into an optical fiber with an integrated fiber isolator (FI) and a fiber splitter (FS) producing the two manipulation beams. (d) The dual beam trap is formed between the flat output facets of two single-mode fibers. (e) Etching of the fiber facets into a lens shape allows for additional modifications of the size and position of the foci of the output optical beams. (f) Using multimode fibers with properly structured illumination, complex beam patterns can be formed behind the output facets of the fibers. As illustrated by Fig. 19, it is advantageous if the focal planes of the two counter-propagating beams are spaced apart and do not overlap.

utilized.

More detailed theoretical analyses carried out for a dual-beam trap formed by counterpropagating Gaussian beams have revealed that if the axial positions $\pm z_{0}$ of the beam waists $w_{0}$ (see Fig. 3), measured with respect to the mid-plane between the focal planes of the two beams, do not fulfill $\left|z_{0}\right| \sqrt{3}<\pi w_{0}^{2} / \lambda$, two maxima of optical intensity are formed along the common beam axis. However, the possibility of independent trapping of two particles in each maximum depends on the particle size, as shown in Fig. 19. Using the approach of ray optics, additional extended studies of this trapping configuration were performed for larger particles by Roosen et 
al. [241-243].

(a)

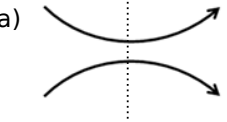

(c)
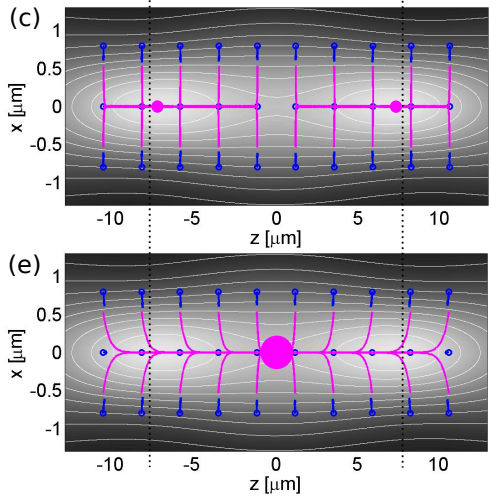

(b)

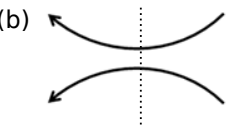

(d)
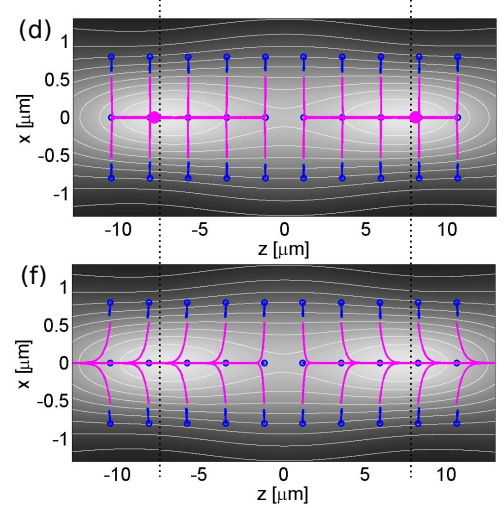

Fig. 19. Calculated behavior of particles of different sizes in two counter-propagating Gaussian beams with longitudinally displaced beam waists of the same radius. The respective beam waists $w_{0}$ of both beams are reached either (a) before or (b) after the beams pass the mid-plane located at $z=0$. Particle trajectories (magenta curves) and optical forces (blue curves) for nanoparticles of radius $a=10 \mathrm{~nm}$ are shown in (c) and (d). The filled magenta circles denote the equilibrium positions (optical traps) located close to the beam waits. Parts (e) and (f) show the same quantities as (c) and (d) calculated for larger particles of radius $a=500 \mathrm{~nm}$. In contrast to the smaller nanoparticle case, only a single optical trap is obtained with the beam configuration (a), while stable trapping is not possible with the beam configuration (b). The following parameters were used in the computations: laser vacuum wavelength $\lambda_{0}=532 \mathrm{~nm}$, refractive indices of the particle and surrounding medium $n_{p}=1.59, n_{m}=1.33$, Gaussian beam waist radii $w_{0}=1 \mu \mathrm{m}$, laser power of each beam $P=1 \mathrm{~W}$, the beams waists placed at $z=z_{\mathrm{R}}= \pm 7.9 \mu \mathrm{m}$, indicated by vertical dotted lines.

Somewhat unexpectedly, it is possible to trap more than two particles in the dual-beam geometry. As independently reported in [244] and [245], several particles tend to self-arrange between the two beam focal planes owing to the phenomenon referred to as longitudinal optical binding. The resulting self-organized colloidal structure is then created and maintained by the forces arising from the radiation scattered by the particles [140,141,246]. If the relative intensities of both beams are carefully tuned, bi-directional transport of multiple particles in such dual-beam configurations can be obtained. These pioneering results were subsequently followed by detailed studies of one-dimensional self-arrangement of particles in Gaussian beams [247-251] and Bessel beams [252-254]. The principle was also adopted to dynamical control of the size and shape of multidimensional optically bound matter [254] that was utilized as a tunable soft-matter optofluidic waveguide assembled by light [255].

Since the dual-beam trapping geometry does not require tightly focused laser beams, optical trapping and transport of particles in air [256,257] or in vacuum [258] is possible. A scheme using three independent pairs of counter-propagating laser beams oriented along three mutually perpendicular axes was also used in the experimental demonstration of laser cooling of atoms [259].

\subsubsection{Standing waves and optical conveyor belts}

If the two counter-propagating beams are mutually coherent and have the same polarization, they interfere, creating a standing wave with the distance between neighboring intensity maxima (fringe width) equal to $\lambda / 2$, where $\lambda$ is the wavelength of light in the medium. In such an 
interference structure, very steep longitudinal intensity gradients are formed; thus, in principle, there is no need to use a tightly focused beam to achieve the longitudinal confinement of the illuminated particles. Since the used Gaussian beams can be wide and, consequently, have long Rayleigh lengths [260] (see Eq. (14)), up to hundreds - or even thousands - of standing wave optical traps of comparable strength can be simultaneously formed. Due to the steep longitudinal intensity gradients, these standing wave traps are especially useful for the 3-D confinement of nanoparticles, providing longitudinal trapping forces that are orders of magnitude larger than those of the single beam optical tweezers [134,261,262]. A simple, practical experimental geometry uses retro-reflection of an incident laser beam from a planar mirror to obtain a well aligned standing wave formed by interference of the incident and reflected beam [262,263].

Because of the axial periodicity of the standing wave intensity distribution, particles of varied sizes and refractive indices display different behavior upon illumination with this periodic pattern. In particular, a particle can be trapped with its center located in the maximum or minimum of the fringe intensity, or - for certain particle sizes - it can move freely across the fringes, without feeling any longitudinal optical forces [264] (compare particles A, B, and C in Fig. 20a). Intuitively, this behavior results from the competition between the optical gradient forces produced by the neighboring fringes. As shown in Fig. 20b, the maximal value of the optical force along the beam propagation direction reaches a plateau for larger particles with radii approximately fulfilling $a>0.6 \lambda$, which overlap with two or more interference fringes. For the combinations of particle sizes and refractive indices lying in the vicinity of the dashed curves shown in Fig. 20b, the longitudinal gradient forces of adjacent fringes cancel out and such a particle does not feel the intensity modulation of the standing wave. Similarly, a particle much larger than the trapping light wavelength overlaps tens of interference fringes and its behavior is determined by the slowly-varying intensity envelopes of the two counter-propagating beams, analogously to the case of the classical dual-beam trap with non-interfering beams discussed in Section 3.3.1. An example of this experimental arrangement is represented by optical macro-tweezers that uses an incident and retro-reflected Gaussian beam with spatially separated focal planes and enables manipulation with large, actively swimming microorganisms of size up to 50-100 $\mu \mathrm{m}[265,266]$.

Besides Gaussian beams, interfering vortex beams or propagation-invariant beams have been also considered for standing wave trapping [162,267], as illustrated in Fig. 21. Such optical interference fields with more complex spatial profiles of intensity and/or phase introduce additional features to the optically induced particle transport. For example, with the use of Laguerre-Gaussian (vortex) beams, the particles are confined off-axis, within a high-intensity ring. Due to the angular momentum carried by the vortex beams, the particles do not move along straight trajectories, but instead follow helical paths around the beam axis (Fig. 21b). When counter-propagating Bessel beams of zeroth order are adopted to form the standing wave, the particles can be confined both on- and off-axis, in a set of concentric bright rings (Fig. 21c).

An optical conveyor belt is a special case of moving standing wave configuration, in which optically confined nanoobjects or cold atoms follow the motion of the interference fringes $[268,269]$. The movement of the fringes can be obtained by adjusting the phase of one of the two interfering beams either by the mechanical motion of a mirror or by a frequency change introduced with an acousto-optical modulator. Using propagation-invariant Bessel beams, nanoparticles can be transported over distances of several millimeters (see Fig. 22). Due to the self-healing property of Bessel beams [270], the beam profile along the propagation axis is only minimally affected by the presence of the transported particles [107,162]. Instead of using the counter-propagating beam geometry, co-propagating, mutually coherent Bessel beams of different axial wavevenumbers can be adopted to form an interference structure, in which particles can be localized and transported bi-directionally if the phase difference between the two beams is modified [108] (see Fig. 21d). This mechanism is also referred to as an active tractor beam $[14,109]$.

When a particle is moving through a viscous liquid, a drag force is acting on it that is 


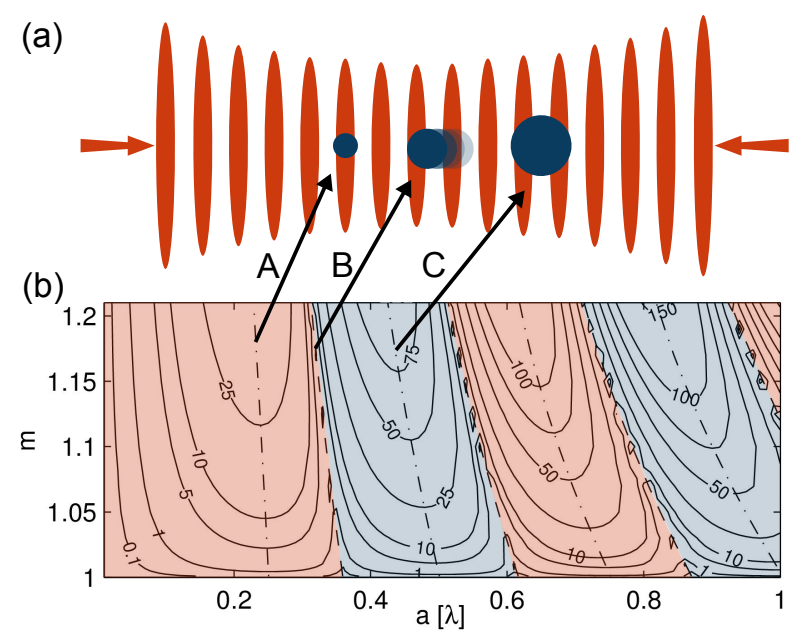

Fig. 20. Confinement and transport of particles in an optical standing wave. (a) A standing wave created by the interference of two counter-propagating mutually coherent beams. According to their sizes and refractive indices, illuminated particles can be confined with their center located either in the high-intensity (particle A) or low-intensity (particle C) regions of the interference fringes. Particles of certain sizes (particle B) are only confined radially on the optical axis, but do not feel the axial intensity modulation of the standing wave. (b) A contour plot of the calculated amplitude of the axial optical force as a function of the particle radius $a$ (in the units of the trapping wavelength $\lambda=\lambda_{0} / n_{\mathrm{m}}$ in the medium) and the relative refractive index of the particle $m=n_{\mathrm{p}} / n_{\mathrm{m}}$. The following parameters were used in the calculations: Gaussian beam waist radii $w_{0}=2.5 \lambda, \lambda_{0}=1064 \mathrm{~nm}$ and laser power of each beam $P=1 \mathrm{~W}$. The dash-dotted and dashed curves represent the loci of the maximum and minimum values of the trapping force magnitude for a given value of $m$, respectively. The numbers labeling the contours denote the force in $\mathrm{pN}$. If the positions of the interference fringes are shifted by a phase change or by frequency detuning of one beam, particles $\mathrm{A}$ and $\mathrm{C}$ follow the moving fringes, thus being transported in analogy with a classical conveyor belt.

proportional to the particle speed and size and the viscosity of the liquid. This drag force effectively tilts the periodic trapping potential associated with the standing wave. The resulting situation is similar to the transport of particles in a standing wave created by interference of counter-propagating beams with unequal intensities, in which a residual scattering force acts on the particles in the longitudinal direction. For a small tilt of the potential landscape and deep standing wave traps, the particle remains confined within a single fringe and moves along with it, acting as the so-called Brownian surfer. On the other hand, for larger tilts and shallower traps, the particle can easily overcome the potential barrier separating the adjacent traps and jump to the neighboring potential minimum. With an increasing rate of such jumps, the particle enters the regime of the so-called Brownian swimmer [271]. The speed of the particle transport $v$ reaches a maximum between these two limiting transport regimes and can be estimated from the following equation [271,272]:

$$
v \equiv\left\langle\frac{\mathrm{d} x}{\mathrm{~d} t}\right\rangle=u-\frac{L k_{B} T}{\gamma} \frac{\exp \left(\frac{\gamma u L}{k_{B} T}\right)-1}{\int_{0}^{L} \int_{y}^{y+L} \exp \left(\frac{U(z)-U(y)+(z-y) \gamma u}{k_{B} T}\right) \mathrm{d} z \mathrm{~d} y}
$$

where $U(x)$ is the periodic potential profile, $L$ is the period length along the $x$-axis, $\gamma$ is the drag coefficient of the particle, and $u$ is the speed of the standing wave motion. An application of this 


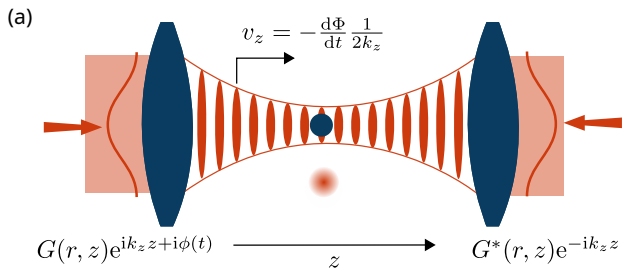

(c)

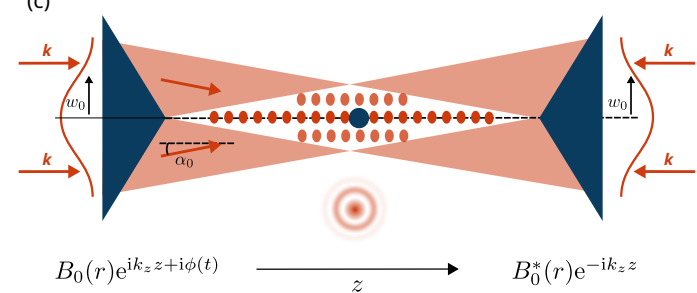

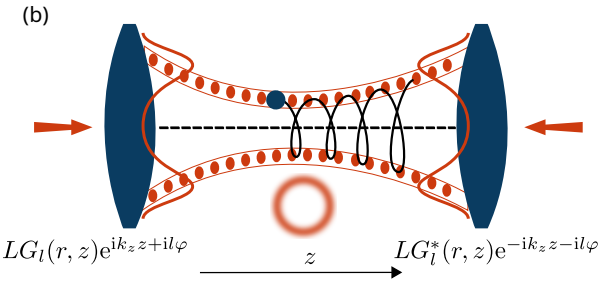

(d)

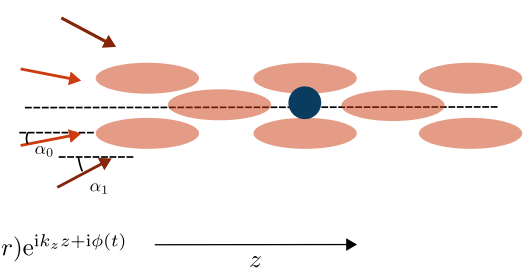

Fig. 21. Optical transport of particles in various types of standing waves formed by (a) counter-propagating Gaussian beams, (b) counter-propagating Laguerre-Gaussian beams, (c) counter-propagating zeroth-order Bessel beams, and (d) co-propagating Bessel beams. In the configuration shown in (a), the fringes move with a longitudinal velocity $v_{z}$, following temporal variations of the phase $\phi(t)$ of one of the beams. For sufficiently low values of $v_{z}$, the trapped particle follows the fringe, in which it was originally confined, acting as a Brownian surfer. For higher values of $v_{z}$, the particle does not follow a single fringe; instead, it frequently jumps between adjacent fringes, against the direction of motion of the fringe pattern. Thus, the particle lags behind the moving fringes and acts as a Brownian swimmer. By inverting the sense of the phase change, one can obtain bi-directional transport.

principle for particle transport in an evanescent-wave conveyor belt is shown in Fig. 38.

A conveyor belt based on the counter-propagating Gaussian beams has been also tested for the delivery of cold atoms [273] over macroscopic distances preserving the atomic coherence with a slight reduction of the coherence time [274]. An analogous 1-D optical lattice based on a standing wave formed by interfering Bessel beams was used to transport ultracold atoms over macroscopic distances of up to $20 \mathrm{~cm}$, with fast transport velocities of up to $6 \mathrm{~m} \cdot \mathrm{s}^{-1}$ and accelerations of up to $2600 \mathrm{~m} \cdot \mathrm{s}^{-2}$ [275]. Even at these high velocities, the momentum of the atoms was precisely defined with an uncertainty of less than one photon recoil. This could possibly allow for the construction of an atom catapult with high kinetic energy resolution, which might have applications in novel collision experiments.

\subsection{Other methods for particle transport in continuous bulk media}

Standard optical tweezers themselves represent an archetypal optical tool for 3-D particle transport [7]. They enable spatial confinement of objects with sizes ranging from tens of nanometers to tens of micrometers in an optical trap created in the vicinity of the beam focus. The transport of the trapped particle is achieved by mechanically repositioning the trap in space, either by keeping the trap fixed while moving the surrounding sample chamber with, for example, a micropositioning stage [276], or by keeping the sample chamber fixed while repositioning the beam focus along with the trapped object. In the latter case, the trapping beam can be deflected before the focusing lens [50] by galvano-optical mirrors [277], piezo-mirrors [278], acousto-optical deflectors [279], electro-optical modulators [280], or moving lenses [281,282].

Currently, the highest degree of freedom can be obtained by using reconfigurable phase diffraction gratings dynamically generated by spatial light modulators (SLMs). Optical trapping systems based on SLMs are commonly termed holographic optical tweezers (HOT). They enable generating a variable number of optical traps at multiple lateral and longitudinal positions after the 

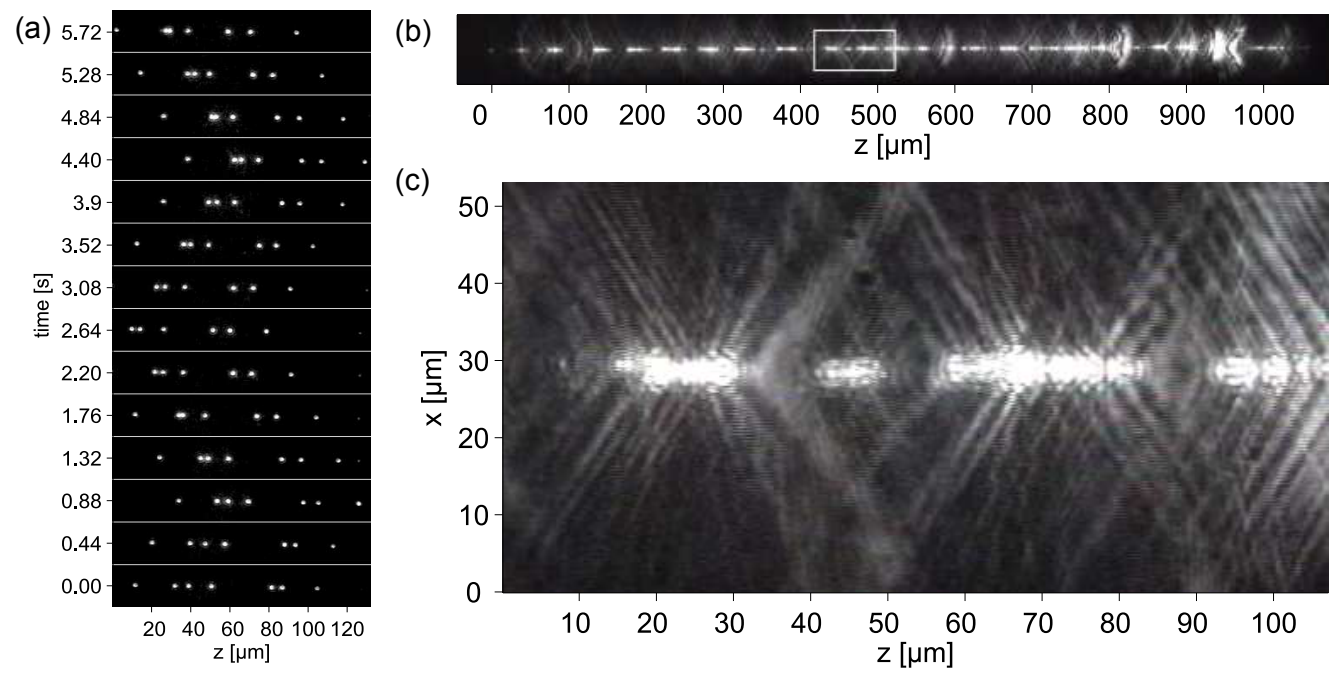

Fig. 22. An experimental example of an optical conveyor belt formed by counter-propagating interfering Bessel beams. (a) Demonstration of bi-directional transport of several simultaneously trapped polystyrene particles of radius $100 \mathrm{~nm}$. (b) An overall view of a one-millimeter long array of simultaneously transported polystyrene particles of radius $175 \mathrm{~nm}$. The image is composed from 10 partially overlapping CCD frames. (c) Detail of the confined particles located within the white rectangle shown in (b). Adapted by permission from Springer Nature Customer Service Centre GmbH: Springer Appl. Phys. B [162]. Copyright 2006

focusing lens $[46,86,88,283,284]$ as well as compensating optical aberrations in the optical path in situ [285]. We refer the reader elsewhere for exhaustive reviews on HOTs [11,50, 52, 74, 87]. The typical bottleneck of HOT-driven particle manipulation and transport is in the user interaction with the setup, because the traditionally used pointing devices, e.g., a mouse or a joystick, are not intrinsically designed to control multiple cursors in 3-D. To overcome this limitation, several research groups have used 3-D joysticks and haptic devices [91, 93, 286] or CCD cameras for detecting the position of each finger of the operator that is subsequently transformed to the desired coordinates of the laser traps [92]. When the number of manipulated objects exceeds the abilities of a single operator, a "Multi-touch console" allows several operators to work simultaneously [287]. "iTweezers" represent a natural consequence of the recent worldwide expansion of touch-screen tablets, which can now be used to view and move particles in the $x y$-plane, while the $z$-coordinate is adjusted by zooming implemented through "stretching" or "compression" of particles between two fingers [90]. A new impulse came from the Kinect technology, which provided a way to read the $x y z$ coordinates of the body skeleton including the hands [94-96]. Since Kinect was primarily designed to capture the whole human body, its capability of recognizing nearby objects (e.g., the hands of a seated human operator) is limited. The latest progress in low-cost 3-D sensors brings new models suitable to track hands in the seated position (e.g., DepthSense 3-D Camera), or even the operator's eyes, to control HOTs [97]. Under certain circumstances, the multiple trapped particles can be also used as miniature handles ("micro-fingers" or "optical grabbers") to manipulate another object that is not directly illuminated by the trapping light $[92,288]$ (see Fig. 23a-b).

A more sophisticated design of the beam-transformation optical system allows the beam to pass the SLM two times, thus enabling to independently shape both the amplitude and the phase of the beam. Using this approach, it is possible to generate tailored phase gradients along the high-intensity light patterns that form the optical traps. Subsequently, the scattering force that 
results from the phase gradient imposed across spatially extended optical traps [289] can be harnessed to push the trapped object along a well defined path across these traps [290] (see Fig. 24).

Optical tweezers with various degrees of complexity are frequently used in liquid environments for the contactless manipulation and investigation of the biophysical properties of various biological samples, e.g., viruses, bacteria, yeast and algae cells [35, 73, 76, 291-297]. In BoseEinstein condensates (BECs), $\sim 10^{6}$ atoms were transferred in a single beam trap over a distance of $44 \mathrm{~cm}$ [298] or merged with another BEC generated separately [299]. More details about the cold atom systems can be found elsewhere [259,300-302]. Novel exciting applications of optical manipulation can be found in the emerging area of optomechanics, where a nanoparticle has been trapped and its center-of-mass motion cooled in vacuum in a single beam trap [303] or transported over a distance of $\sim 66 \mathrm{~cm}$ by the mechanical displacement of a fiber optical trap [304]. Finally, beam shaping in multimode optical fibers provides an exciting possibility to avoid bulky lens-based optics and to generate several traps in the vicinity of the fiber output facet (see Fig. 25) [305]. 


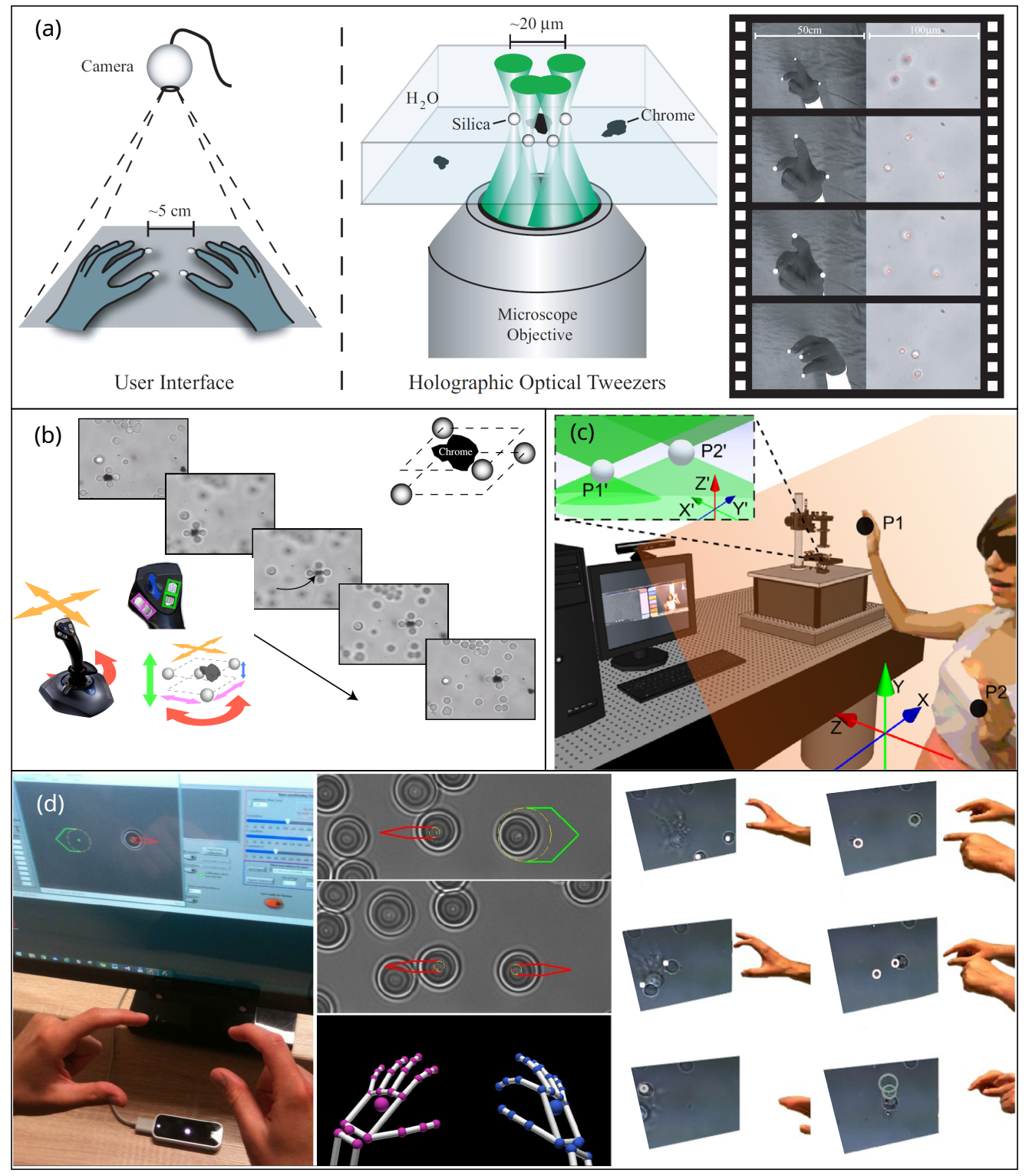

Fig. 23. User-friendly interfaces for holographic optical tweezers. (a) Image analysis software connected to a web-camera traces white markers on the operator's fingers and transfers their positions to holographic optical tweezers trapping multiple microobjects at desired locations. The central panel shows the operating principle of an optical grabber, which uses optically trapped silica microbeads as handles for manipulating a light-sensitive absorbing object (a chrome particle). Adapted with permission from [92]. Copyright 2006 Optical Society of America (b) An optical grabber controlled by a joystick, with the toggle buttons used to change the inter-particle separation. Adapted with permission from [91]. Copyright Deutsche Physikalische Gesellschaft. Reproduced by permission of IOP Publishing. CC BY-NC-SA (c) A Kinect sensor detects the positions of the operator's hands, while the associated hardware and software control the positions of the trapped microobjects. Adapted with permission from [95]. Copyright 2013 IOP Publishing. (d) LeapMotion sensors analyze the positions of the operator's hand joints, while the associated software controls the positions and the off/on switching of individual traps with different operation modes. Adapted with permission from [97]. Copyright 2016 IOP Publishing. 
(a)

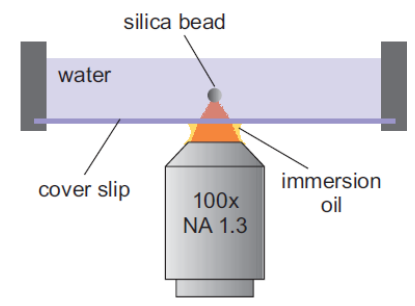

(b)

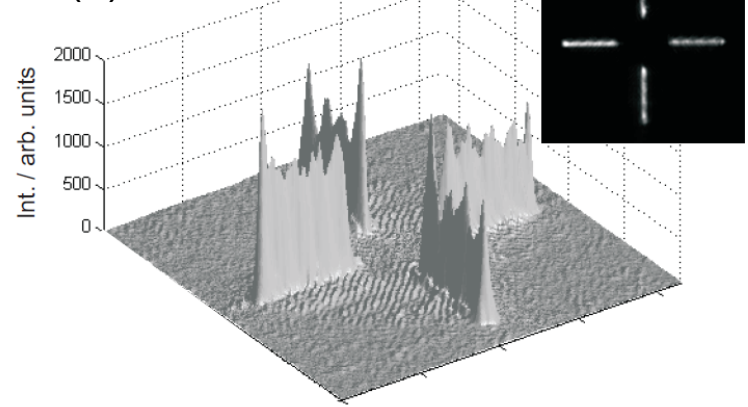

(c)

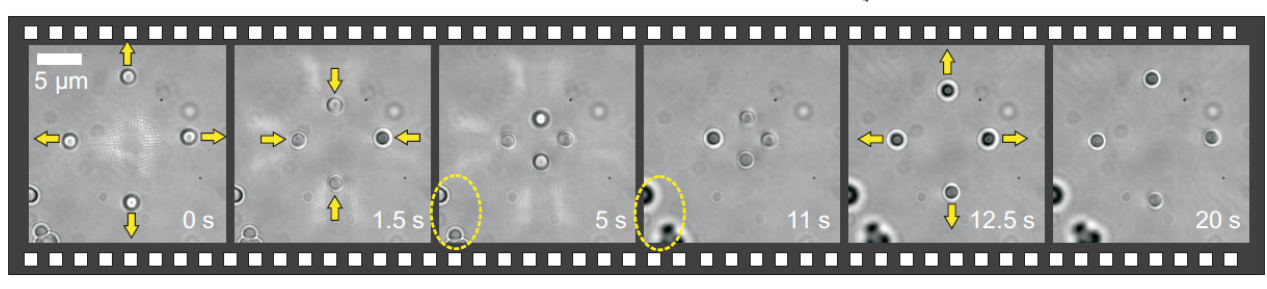

Fig. 24. Transport of particles within optical traps featuring imposed phase gradients. (a) Experimental geometry for creating an optical trap based on an amplitude- and phase-shaped focused laser beam. (b) An example of a three-dimensional reticle-shaped trapping beam with imposed phase gradients along the high intensity regions. (c) Four silica microspheres are confined in the high-intensity regions of the reticle beam and alternatively pushed inward and outward by changing the sign of the phase gradients imposed by an SLM. Adapted with permission from [290]. Copyright 2008 Optical Society of America.
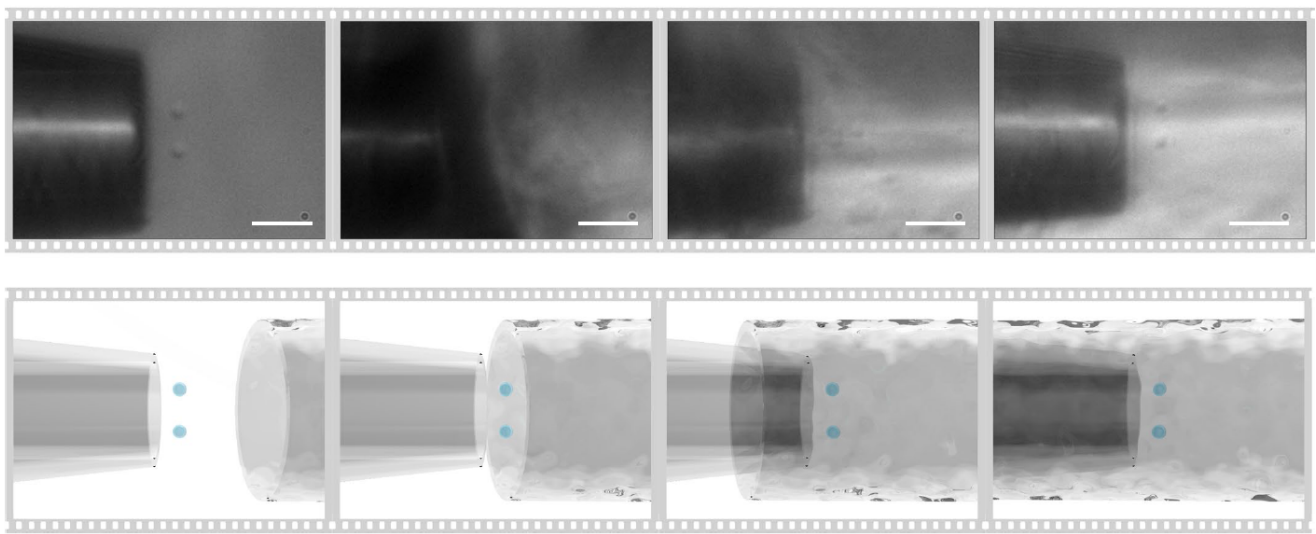

Fig. 25. Multiple holographic optical tweezers delivered through a lensless multimode optical fiber. The pictures (top: experiment, bottom: schematic illustration) show the optical manipulation of two 1.5- $\mu$ m-diameter silica microspheres inside a turbid cavity representing a complex, hard-to-access environment. Scale bars: $10 \mu \mathrm{m}$. Adapted by permission from Springer Nature Customer Service Centre GmbH: Nature Photonics [305], Copyright 2018. 


\section{Interface-constrained transport with optical forces}

The increasing integration of optical elements with microfluidic chips has led to the development of new optofluidic platforms for optical manipulation with enhanced versatility and functionalities for a diverse spectrum of applications in research and clinical laboratories [306,307]. In chipbased optofluidic systems, the presence of interfaces between different phases (solid, liquid, and gas) can lead to an additional confinement of transported particles, restricting the free 3-D motion of the particles to lower dimensions. As we discuss below, in a typical chip-based experimental system, the confinement of particles can be imposed near a liquid-solid or liquid-liquid interface. Alternatively, interfaces between air and liquid or solid phases can be also exploited. Near the interface, transport of particles can be initiated by both radiative and evanescent waves. In general, if the wavevector of the incident optical field has a component directed along the interface, the associated scattering force moves an illuminated particle predominantly in this direction. Thus, the principles of optical propulsion of particles described in Section 2 can be used for transporting the particles along the interface, provided that they do not stick to it . In the case of radiative waves, such interfacial transport requires a beam incident upon the interface with a non-zero angle with respect to its normal.

\subsection{Incident radiative waves}

(a)

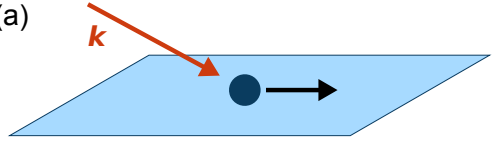

(c)

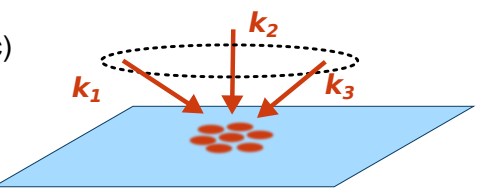

(e)

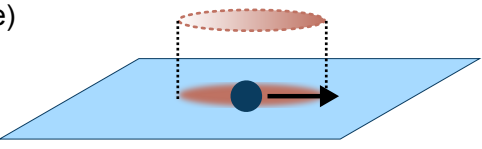

(b)

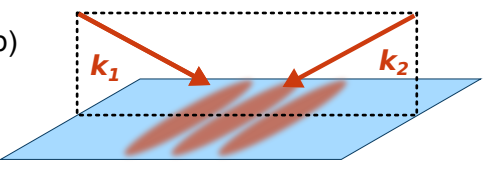

(d)

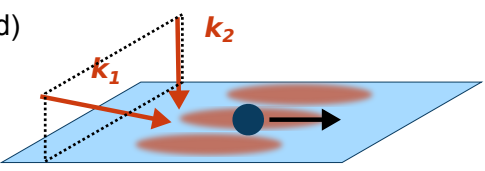

(f)

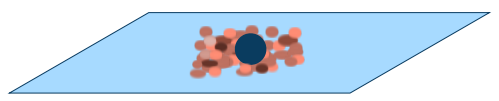

Fig. 26. Interface-constrained guiding of objects under different configurations of incident radiative waves. (a) A single beam incident at an angle on the surface guides the particle along the wavevector component parallel to the surface. (b) Two and (c) three (or more) beams incident under different angles form an interference lattice at the interface, in which the particles can be localized. Phase changes in one of the beams lead to a movement of the lattice, following the conveyor belt principle. (d) If there is a net wavevector component parallel to the surface, the particles are transported along the fringes by the scattering force. (e) When a phase gradient is imposed along the high-intensity fringe pattern with an SLM, the particle moves along this gradient, in a way similar to (d). (f) Speckle represents a special example of a random interference field of multiple mutually coherent beams that can be used to guide particles.

Figure 26 summarizes various configurations exploiting a single or multiple radiative waves for particle transport along an illuminated interface. A radiative wave incident upon an interface between two materials with different index of refraction is partially reflected, with the amplitude of the reflected wave depending on the surface reflectivity and the angle of incidence. For example, 
a typical water-glass interface reflects $\sim 0.4 \%$ of the normally incident power. In the direction perpendicular to the surface, the coherent incident and retro-reflected waves interfere and form a standing wave, which - as shown in Fig. 15c - might assist with the localization of an illuminated object above the surface [262,263]. In the direction parallel to the surface, interference of two (Fig. 26b,d) or more (Fig. 26c) radiative waves incident at an angle forms high-intensity interference fringes or lattices [308], in which multiple particles can be localized [246,309-314]. These geometries are referred to as interferometric optical tweezers, even though the optical trapping is predominantly two-dimensional, with the surface repulsive forces counter-balancing the radiation pressure of the incoming waves. Applying a phase shift to one of the interfering beams causes the motion of the interference pattern together with the particles confined in it, according to the operating principle of the optical conveyor belt (Section 3.3.2). As discussed in Section 3.3.2, if a particle overlaps two or more interference fringes, its size and refractive index dictate whether the particle settles with its center localized in an intensity maximum or minimum, or - for specific combinations of the size and refractive index - does not feel any optical force from the standing wave [264] (see Fig. 20). This feature has been used for the selective transport of particles based on their size, shape or optical properties [16, 103, 106,315-319], and the method is also referred to as passive optical sorting [21,104].

\subsubsection{Planar liquid-solid interfaces}

The majority of the configurations described in Fig. 26 have been implemented near planar water-glass interfaces. Various examples of selective transport - or sorting - of particles at such interfaces are provided in Figs. 27, $28,29$.

(a)

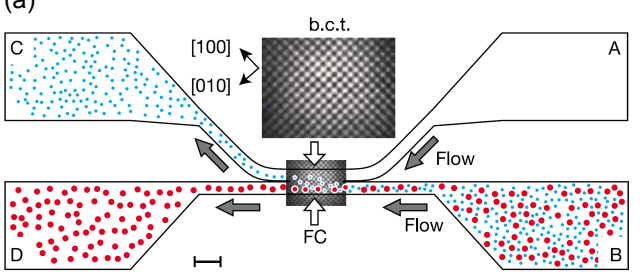

(b)

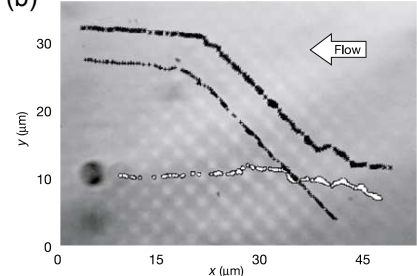

Fig. 27. Optical sorting of particles flowing through an optical lattice created by 5 interfering beams (geometry of Fig. 26c). (a) A mixture of particles of different properties (size or refractive index) flows from chamber $\mathrm{B}$ through the optical lattice (FC), where the particles are deflected to chambers $\mathrm{C}$ or $\mathrm{D}$. The scale bar corresponds to $40 \mu \mathrm{m}$. (b) Sorting of protein capsules with diameters of $\sim 2 \mu \mathrm{m}$ (black trajectories) and $\sim 4 \mu \mathrm{m}$ (white trajectory). While the trajectory of the $4 \mu \mathrm{m}$ capsule remains virtually unaffected by the optical lattice, the $2 \mu \mathrm{m}$ capsules are deflected at an angle of $\sim 45^{\circ}$ with respect to the flow direction due to optical forces. Experimental parameters: flow speed $20 \mu \mathrm{m} . \mathrm{s}^{-1}$, total laser power $530 \mathrm{~mW}$. Adapted by permission from Springer Nature Customer Service Centre GmbH: Nature [16], Copyright 2003

The presence of an asymmetric, periodic optical potential that is externally modulated in a time-dependent fashion constitutes the principal ingredient for implementing the so-called optical ratchets [320,321], which belong to the family of Brownian motors [271,322]. With such dynamic systems, it is possible to exploit stochastic forces of thermal or artificial origin for long-range directed particle transport. An effective asymmetric optical potential experienced by an illuminated particle can be formed, for example, by scanning of a single laser beam sufficiently fast [320] or by dynamically switching between several different configurations of the beam foci [321,323]. Alternatively, deterministic optical ratchets can be obtained by combining oneor two-dimensional periodic arrays of asymmetric optical potential wells. These can be produced, 


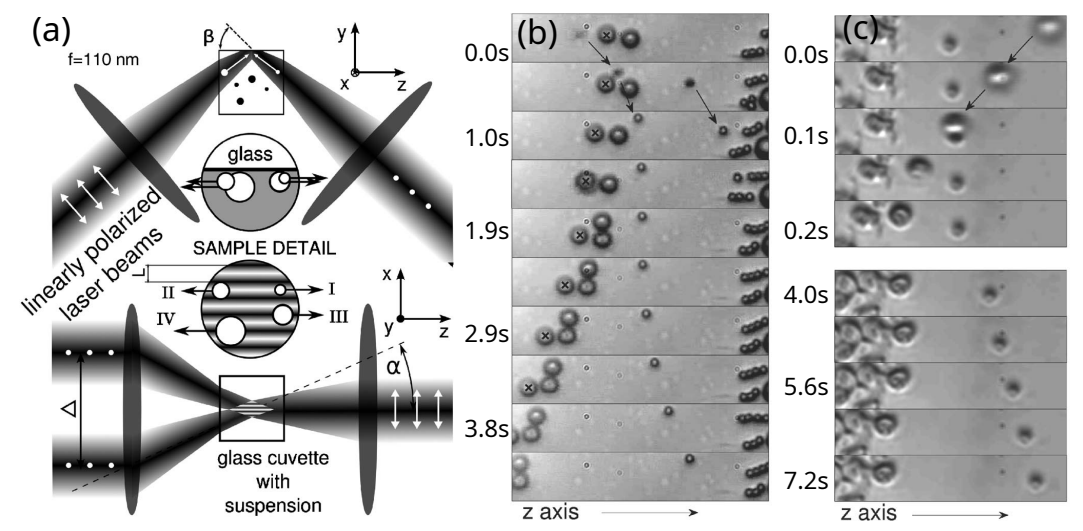

Fig. 28. Optical sorting of particles with a static interference field in a static liquid. (a) Experimental setup. Three linearly polarized laser beams are focused with lenses into an optical glass cuvette. The beam polarization directions are indicated with white arrows and dots and are controlled by polarizing beamsplitters combined with half-wave plates. All three beams overlap at the top surface of the cuvette and form a sorting region of 40x60 $\mu \mathrm{m}^{2}$ elongated along the $z$-axis. This is the same geometry as that shown in Fig. 26c, where the beam propagating from the right does not interfere with the other two beams propagating from the left. The distance between the interference fringes formed along the $x$-axis by the two beams coming from the left is given by $L=\lambda /(2 \sin \alpha \cos \beta)$, where $\lambda$ is the laser wavelength in the medium and the angles $\alpha$ and $\beta$ are defined in the figure. The fringe width $L$ can be adjusted by changing the distance $\Delta$ between the beams. The insets indicate the behavior of objects of four different sizes in the interference fringes. (b) Time sequence of CCD images of the top surface of the cuvette showing sorting of polystyrene microspheres with the diameter of $1 \mu \mathrm{m}$ (type I) and $5.2 \mu \mathrm{m}$ (type IV). (c) Sorting of living yeast cells (larger) and spores (smaller). Reprinted from [106], with the permission of AIP Publishing.

for example, by combining two mutually displaced optical lattices with the periodic motion of the sample chamber containing the particles suspended in a liquid [324,325]. Upon periodic modulation, multiple particles, which simultaneously experience optical and hydrodynamic forces, can be guided along a specific direction within the ratchet structure; this direction depends on the geometry of the two-dimensional lattices forming the ratchet and on the speed and extent of the periodic actuation of the sample chamber position [326] (see Fig. 30).

In addition to the periodic interference structures discussed above, optical speckle patterns (see Fig. 26f) have been also investigated for particle transport, as they provide random optical potential landscapes whose statistical properties fundamentally differ from those of periodic potentials. For example, in non-equilibrium statistical physics, the dynamics of a Brownian particle in a moving periodic potential can be described as a straightforward generalization of the dynamics of a Brownian particle in static equilibrium potentials, while this is no longer the case for random potentials, for which a full non-equilibrium description is required [327]. The motion of objects in random potential landscapes occurs in multiple natural phenomena ranging from the mobility of organelles within a biological cell [328] to the diffusion of stars within a galaxy [329]. However, in the context of optical imaging and manipulation, random speckle patterns were considered for a long time as an unwanted result of propagation of coherent light through optically heterogeneous media. Optical speckle results from the interference of a large number of waves propagating along different directions and with a random phase distribution. These random-phase waves in turn arise from the complex scattering of light in optically heterogeneous media, such as biological tissue, turbid liquids, colloidal suspensions, and rough surfaces (diffusers). Despite their random appearance, they share some universal statistical properties [330], which can be 


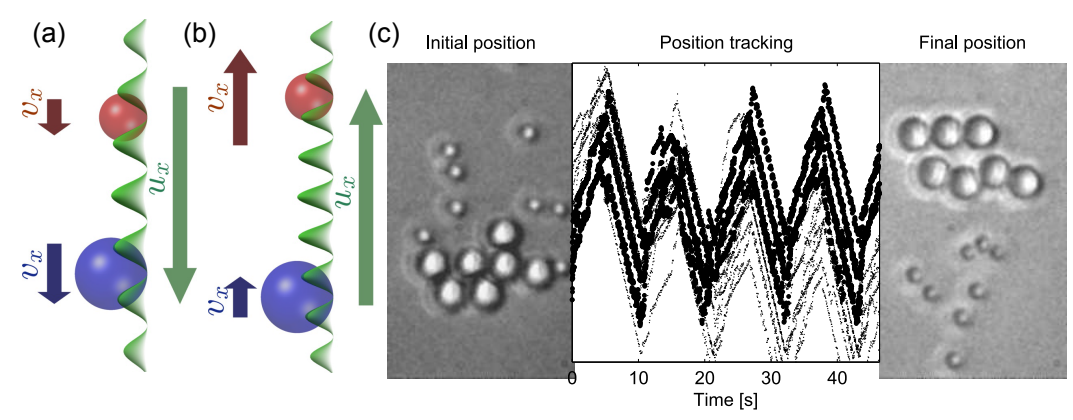

Fig. 29. Optical sorting of particles with a movable interference field in a static liquid (geometry of Fig. 26b). Different fringe spacings are used for moving the fringe pattern (a) downward and (b) upward. Particles of different sizes $(0.8 \mu \mathrm{m}$ - red and $1.6 \mu \mathrm{m}$ - blue) are dragged with different velocities. (c) After several repetitions of the fringe modulation cycle, the difference in particle velocities results in the spatial separation of the particles. The same principle has been used to separate algal cells with different internal structures as well as to sort spherical and spheroidal objects. Adapted with permission from [318]. Copyright 2014 Optical Society of America.

exploited to control and manipulate microscopic particles. The idea of using such disorder to confine small particles came initially from the atom cooling community [331,332]. The first experimental approach used a speckle field to implement a far-detuned dipole trap for pre-cooled atoms [332]. Alternatively, the speckle field itself was used to simultaneously cool and trap the atoms [331,332]. At the mesoscopic scale, random optical potentials generated by speckle patterns have been investigated in connection with the motion of Brownian particles through them. Both static and time-varying speckle fields have been related to the emergence of anomalous diffusion in colloidal suspensions [333-338]: transient subdiffusion has been observed in static one-dimensional and two-dimensional random energy landscapes [333, 335, 336, 339], while a regime of transient superdiffusion emerges if the random field is fluctuating in time [334,340]. Near a liquid-glass interface, a moving speckle pattern has been employed to sort Brownian particles of different sizes (see Fig. 31), with the possibility of tuning the sorting parameters by changing the temporal and spatial statistical properties of the speckle pattern [337,341]. Interestingly, even though the fast fluctuations of the incident optical field do not influence the motion of a Brownian colloid at the single-particle level, they can be used to control effective dispersion forces between small particles [342]. Similarly, recent theoretical works on optical binding between dipolar particles under non-coherent random illumination [343,344] and on dipolar particles near fluctuating light sources [345,346] have suggested striking similarities between dipolar optical forces in random fields and Casimir interactions.

Finally, let us briefly note that the illumination of particles suspended in a liquid with an optical vortex beam [347], or with a rotating interference field [348], leads to the circular motion of the particles over the illuminated surface [349]. A similar effect was observed with metallic nanoparticles, optically bound in a plane parallel to a solid-liquid interface, when they were illuminated with a circularly polarized wide beam. The sense of the particle circulation than depended on the number of particles and the shape of the structure [350]. Subsequently, this circular motion pattern can induce directed fluid flow, thus acting as an optical micropump. In vortex beams, an azimuthal gradient of the optical phase produces an azimuthal force inducing the circular motion of an illuminated particle. This principle can be generalized by imposing azimuthal phase gradients on arbitrarily shaped distributions of optical intensity, resulting in the possibility of creating any on-demand two-dimensional trajectory in the vicinity of the surface for dielectric [351] or plasmonic [352] particles (see Fig. 32). 


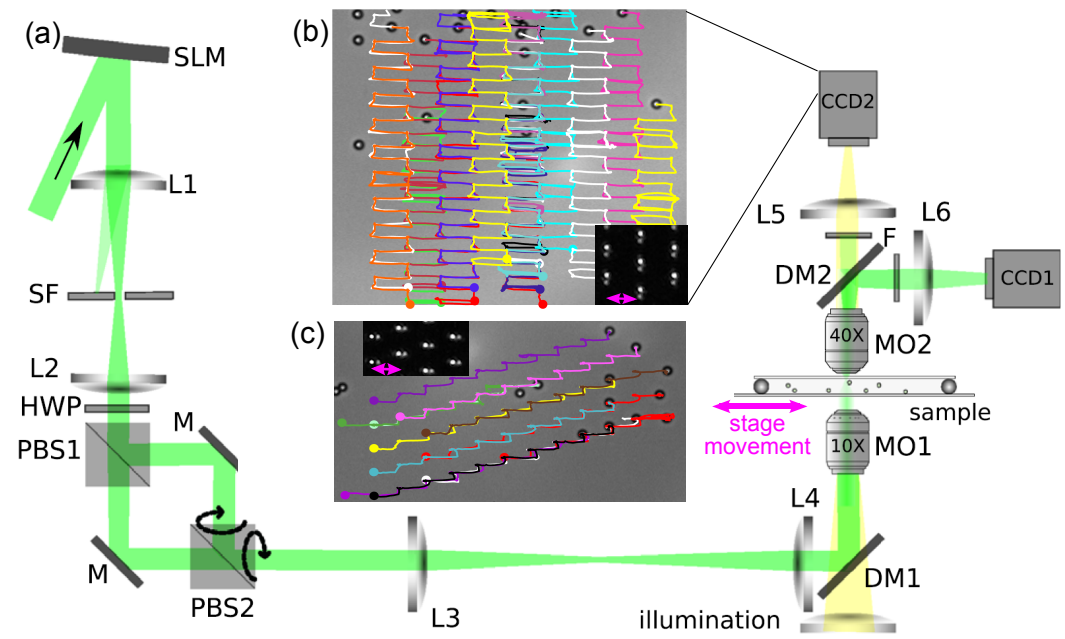

Fig. 30. Transport of microparticles using two-dimensional deterministic optical ratchets. (a) Experimental setup. An incident laser beam is reflected from a spatial light modulator (SLM) that creates an optical lattice. Subsequently, this lattice is replicated by polarizing beam splitters (PBS) into two lattice clones with mutually orthogonal polarizations and variable relative intensities and spacing. Together, they form an asymmetric periodic optical potential. The position of the sample chamber containing suspended particles can be modulated using a piezo-stage, which effectively makes the optical potential time-dependent. L - lenses, SF spatial filter, HWP - half-wave plate, M - mirrors, DM - dichroic mirrors, MO - microscope objectives, F - attenuation filters, CCD - cameras. (b) Transport of particles in the direction perpendicular to the stage motion. (c) Transport of particles at an oblique angle with respect to the direction of the stage motion. In (b) and (c), the color curves denote the trajectories of individual particles from their initial position (colored dots) to the final ones (images of the particles). In addition, the insets show the structure of the underlying optical lattices, with the magenta arrows indicating the direction and amplitude of the stage motion. Adapted with permission from [326]. Copyright 2017 by the American Physical Society. 


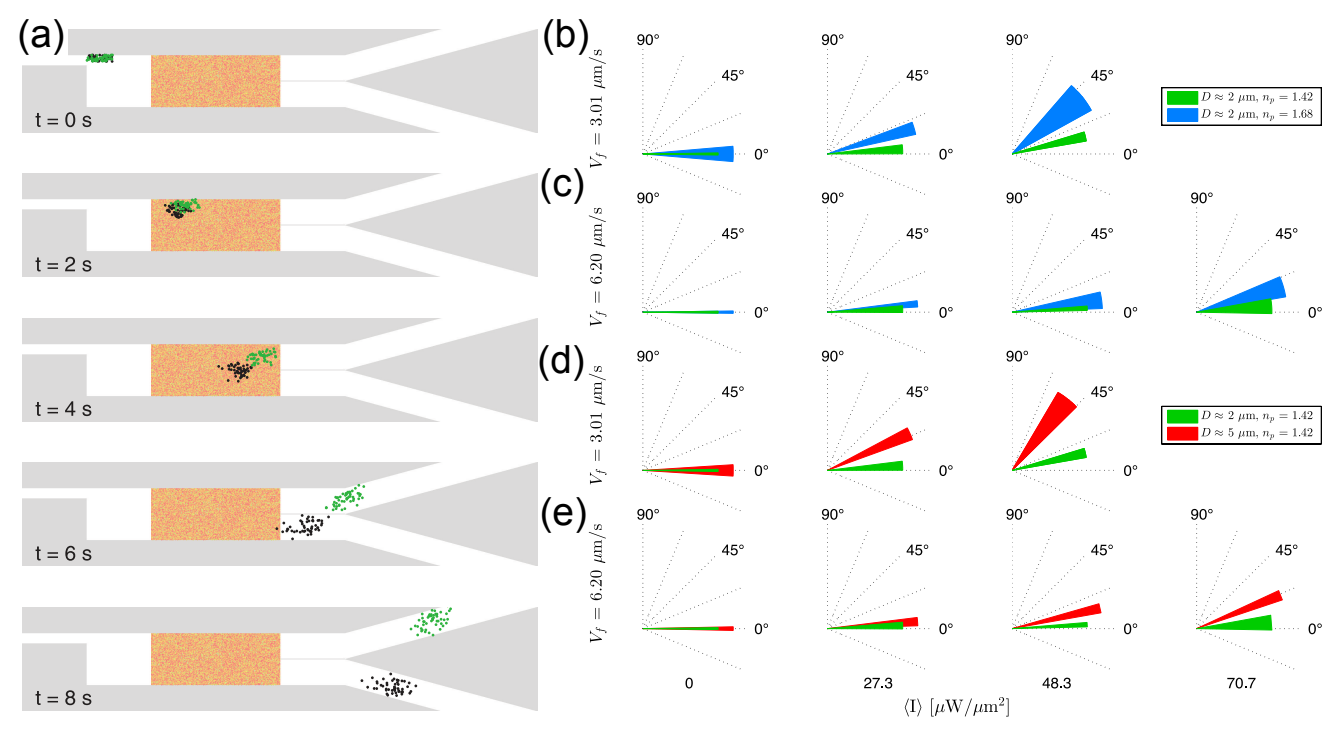

Fig. 31. An example of sorting of colloids flowing in a microfluidic channel using ratcheting speckle fields. (a) Principle of the method. An incoming mixture of colloids is separated when passing through a speckle pattern (orange area) moving slowly downward and fast back upward. Depending on the particles' properties (e.g., size, refractive index) a different optical force acts on them and separates them into different output channels. Adapted with permission from [337] under CC BY 3.0. (b-c) Experimental angular distribution of two classes of particles with similar diameter $(D \simeq 2 \mu \mathrm{m})$ but different refractive index ( $n_{\mathrm{p}}=1.42$, green areas, and $n_{\mathrm{p}}=1.68$, blue areas) in a microfluidic speckle sorter for flow speeds $V_{f}=(3.01 \pm 0.12) \mu \mathrm{m} . \mathrm{s}^{-1}$ in (b) and $V_{f}=(6.20 \pm 0.68) \mu \mathrm{m} . \mathrm{s}^{-1}$ in (c) and different average speckle intensities $\langle I\rangle$. The flow is directed along the $0^{\circ}$ line, while the speckle is shifted along the $90^{\circ}$ line. The width of the areas represents one standard deviation of the particle spread around the average value. (d-e) Same as (b-c) with the particle size $(D=(2.06 \pm 0.05) \mu \mathrm{m}$, green areas, and $D=(4.99 \pm 0.22) \mu \mathrm{m}$, red areas $)$ used as the selection parameter, while the refractive index is kept constant at $n_{\mathrm{p}}=1.42$. Adapted with permission from [341]. Copyright 2014 Optical Society of America. 


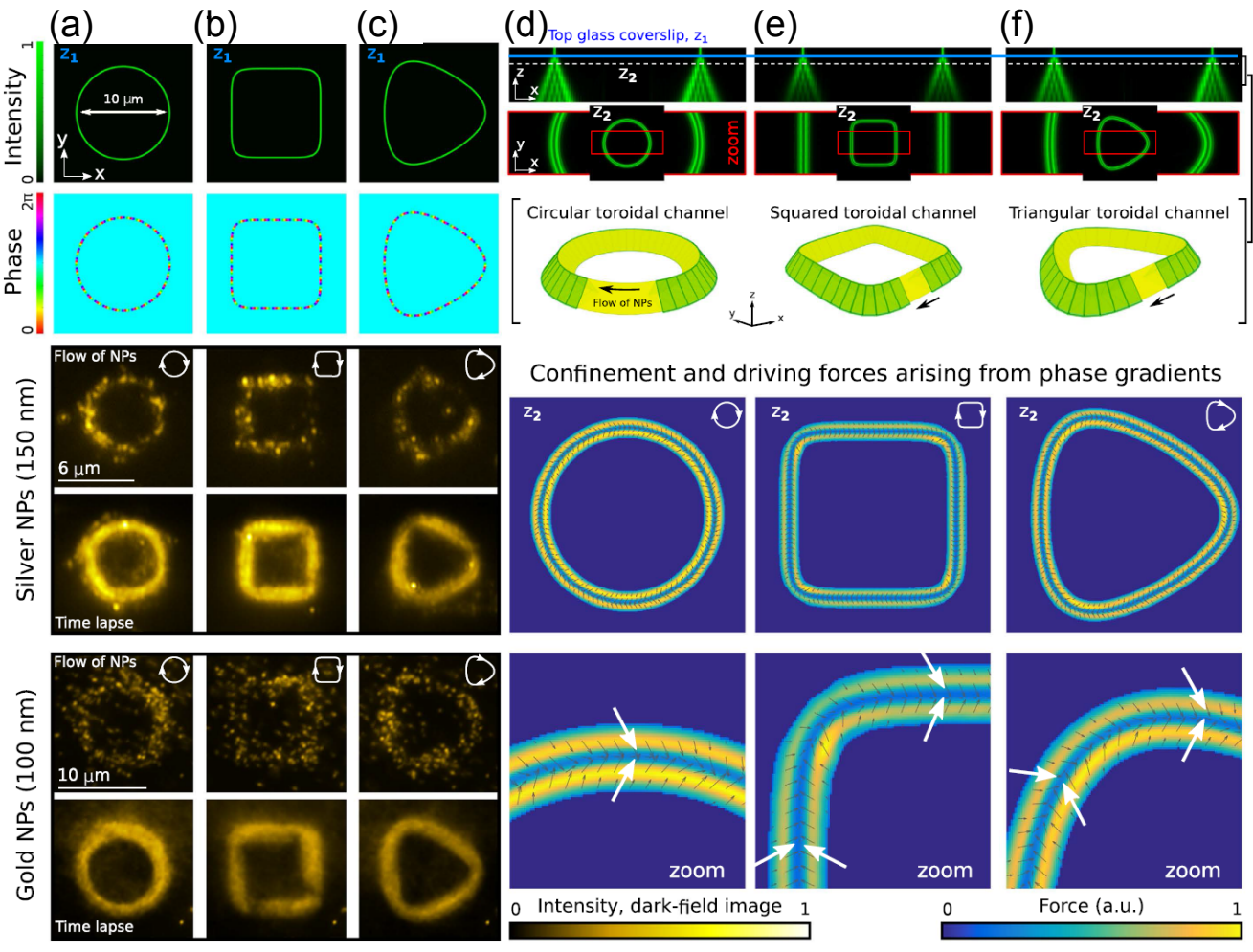

Fig. 32. An example of particle transport along light intensity patterns with imposed gradients of optical phase. The lateral intensity profile is shaped into (a) a circle, (b) a square, and (c) a triangle. First row: intensity profiles, second row: phase profiles. Third and fourth rows: transport of $150 \mathrm{~nm}$ silver nanoparticles along the shaped toroidal channels. Fifth and sixth rows: transport of $100 \mathrm{~nm}$ gold nanoparticles along the shaped toroidal channels. (d-f) The first and second rows show the focusing profiles of the beams forming the toroidal channels in $(\mathrm{a}-\mathrm{c})$. The third and fourth rows show the spatial distributions of the confinement and driving optical forces arising from the phase gradients. Adapted with permission from [352] under CC BY 4.0. 


\subsubsection{Air-liquid interfaces}

Similar to solid-liquid interfaces, surface tension at an air-liquid interface can also serve to counter-balance the scattering force of an incoming light beam, thus stabilizing an illuminated particle along the longitudinal direction. A particle optically trapped near a solid immersed surface can become immobilized by adhesion to the surface and, consequently, cannot be further manipulated by optical forces that are typically much weaker than the adhesive forces. On the other hand, a particle located at an air-liquid interface is partially suspended in the two fluid media and there are no forces restricting its lateral movement across the interface. Hence, optically induced 2-D transport of particles within the plane of the interface is possible with comparably low powers of the incident manipulation beam. To this end, low-NA, low-magnification microscope objectives can be used for trapping, thus allowing for larger fields of view. As in the case of solid-liquid interfaces, multiple particles can be simultaneously manipulated and transported along arbitrary desired trajectories [353] or used as externally driven local probes for investigating interfacial flows. Since the illuminated objects are confined at an interface between two media with different refractive indices, the light beam propagating from air to liquid (or vice versa) refracts, which results in partial transfer of momentum from the light to the object. As in the tractor beam case, this can induce counter-intuitive motion patterns of the objects along the air-liquid interface (see Fig. 33) [354,355]. Particularly exciting possibilities arise when non-spherical particles are manipulated and transported. For example, Fig. 34 shows floating microwedges fabricated by photopolymerization that act as miniature sailboats propelled by light along the interface [356].

(a)

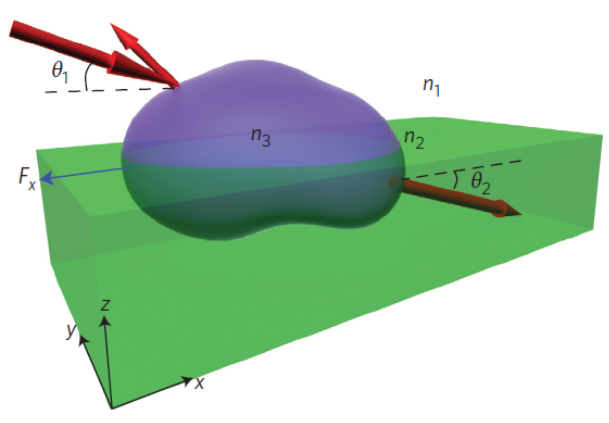

(b)

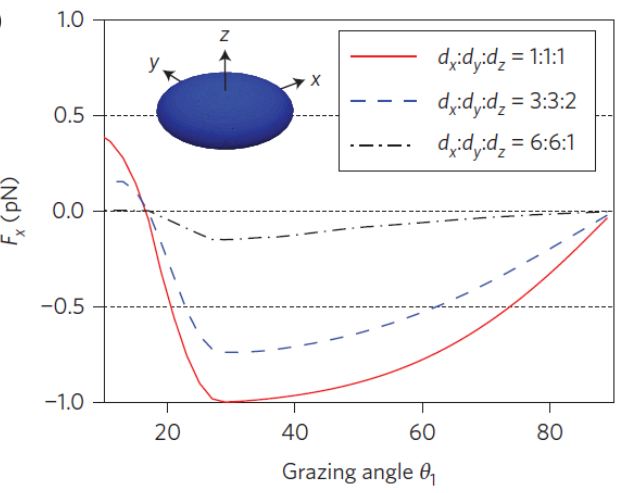

Fig. 33. Amplification of the forward momentum of light accompanied by the backward motion of an illuminated scattering particle located at an air-water interface when the light propagates from air (refractive index $n_{1}$ ) into water (refractive index $n_{2}>n_{1}$ ) through the particle (refractive index $n_{3}$ ). (a) Schematic of the forward momentum amplification. The red arrows represent directional vectors along the incident, reflected and transmitted rays. The lengths of these directional vectors are proportional to the refractive index of the medium in which the rays lie. As a result of momentum conservation, a negative scattering force $F_{X}$ (blue arrow) is exerted on the scattering particle along the interface. (b) The component $F_{x}$ of the calculated scattering force exerted on oblate spheroids with different aspect ratios (see inset) as a function of the grazing angle $\theta_{1} . d_{x}, d_{y}$ and $d_{z}$ denote the lengths of the main axes of the spheroids, with the cross-sectional area along the interface (the $x y$-plane) remaining constant $\left(d_{x}=d_{y}=10 \mu \mathrm{m}\right)$. The forces are calculated with a ray-tracing technique for spheroids with refractive index $n_{3}=1.42$ floating at the air-water interface $\left(n_{1}=1, n_{2}=1.33\right)$ under the illumination with a p-polarized plane wave with irradiance of $10 \mu \mathrm{W} . \mu \mathrm{m}^{-2}$. Adapted by permission from Springer Nature Customer Service Centre GmbH: Nature Photonics [355], Copyright 2013. 


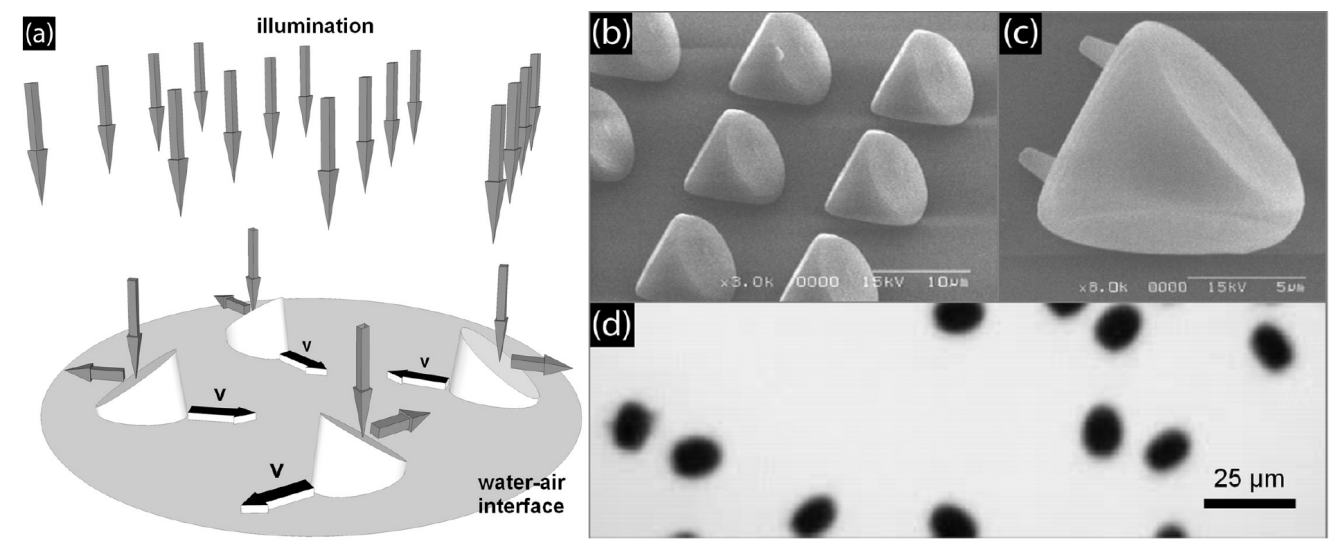

Fig. 34. Transport of microscopic light-driven sailboats along a water-air interface. (a) Schematic of the experimental configuration for the transport of micro-sailboats under illumination perpendicular to the air-water interface. (b) Electron microscope image of the wedges used as the micro-sailboats. These wedges are fabricated on a glass surface via photopolymerization. (c) Detail of the wedge in sailing position. (d) The final sample of moving micro-sailboats observed under an optical microscope. Reprinted from Ref. [356] with the permission of AIP Publishing. 


\subsection{Transport based on optical waveguides}

Alternatively to radiative waves (Section 4.1), non-radiative evanescent waves can also be used to guide particles near an interface. Evanescent waves can be formed, for example, due to total internal reflection of light from interfaces between high- and low-refractive index media illuminated from the high-index side. Such interfaces represent the key ingredient of optical waveguides, which use the repeated total internal reflection of light from a suitably shaped interface between materials with a sufficient contrast of refractive indices for long-distance light routing. Evanescent waves reside on the low-index side of the interface and their intensity exponentially decays with increasing distance from it, with a characteristic decay length comparable to the wavelength of the used light. In the direction parallel to the interface, an evanescent wave induces a scattering force that pushes an illuminated object along the interface; in the direction perpendicular to the interface, the gradient of the wave intensity attracts an object with the relative refractive index $m>1$ towards the interface. The first experimental demonstration of object propulsion due to the optical forces generated by an evanescent field was performed in an aqueous suspension of colloidal particles deposited on a sapphire prism illuminated under total internal reflection conditions (Fig. 35a) [8]. Improved configurations came about with the utilization of channeled optical waveguides, in which most of the mode intensity is confined within the waveguide and the generated evanescent wave interacts with objects located outside the waveguide in the vicinity of its surface (Fig. 35b-d). Besides dielectric objects, metallic nanoparticles $[357,358]$ and living cells $[359,360]$ can be also transported in the same way.

If multiple co-propagating guided modes are excited simultaneously, by controlling either the polarization [358] or the position [361] of the incident beam coupled into the waveguide, larger metallic and dielectric objects can be repelled from the waveguide [358], propelled along its top or side surfaces [358,361], or held in place due to the interference of modes resulting in intensity gradients along the waveguide [361] (Fig. 36). More complicated waveguide geometries can also be devised to separate particles into multiple channels [362,363], to counter-propagate and stop particles [239], or to circulate particles above a micro-ring waveguide [364]. Photonic crystal waveguides that support propagation of "slow light" with a highly reduced group velocity can enhance the magnitude of the evanescent radiation pressure by a suitable choice of the incident wavelength within the slow light spectral region [365]. Furthermore, it has been proposed that optical pulling forces can be generated with the use of carefully designed hollow core double-mode photonic crystal waveguides supporting both the zeroth-order mode with a larger forward momentum and the first-order order mode with a smaller forward momentum [366]. Here, the pulling force results from the conservation of linear momentum during the process of mode conversion by an illuminated scattering particle. Optical forces arising from the evanescent field can be enhanced by cavity effects that result from combining a resonant dielectric waveguide structure with a prism coupler to produce Fabry-Pérot-like cavity modes at a dielectric-fluid interface. [367]. Finally, a sub-wavelength slot waveguide (see Fig. 37) is a promising alternative for the localization and transport of nanoparticles or biomolecules [368].

When counter-propagating mutually coherent evanescent waves are used to generate a standing wave along the waveguide and the relative phase shift between the two waves is controlled, an evanescent conveyor belt is obtained, in which particles can be transported bidirectionally [105,374] (Fig. 35f). The maximal velocity of particle transport by such evanescent conveyor belts was investigated in [272] (see Fig. 38). It was found that due to the combined effects of hydrodynamic coupling and optical binding between suspended particles, multiple particles can be simultaneously transported almost ten times faster than an individual particle [375]. The whole concept was later implemented in an optofluidic chip and applied to the fast determination of the mechanical properties of DNA and molecular motors [376] (see Fig. 39).

An alternative implementation of the waveguide-based conveyor belt used peristaltic transport 

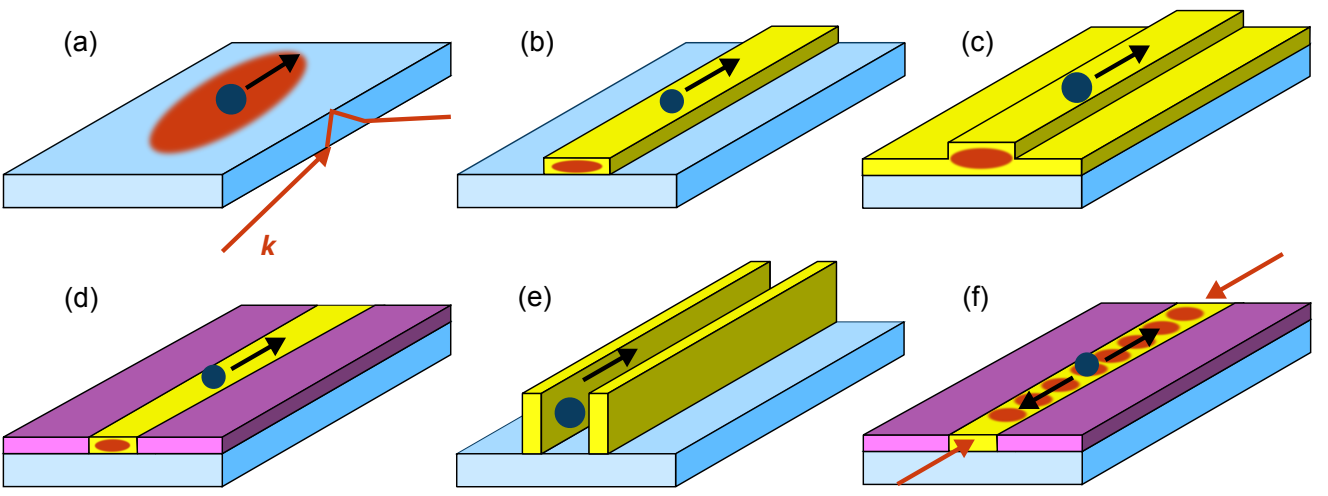

Fig. 35. Typical configurations for optically-induced particle transport with evanescent waves. (a) An evanescent wave generated by total internal reflection from an interface between media with a sufficient contrast of refractive indices propagates in an optically less dense medium and propels the particle over the illuminated area [8,367]. The distance over which the particle can be transported can be significantly increased by fabricating a channeled waveguide on top of the substrate (blue) in the form of (b) a strip [239, 364, 369], (c) a rib [370], or (d) a buried waveguide [359,371,372]. (e) Instead of a planar dielectric structure, a more complex nanostructure can be manufactured on top of the substrate, in which a mode is formed inside a slot waveguide and used to localize and guide the particles within the slot $[368,373]$. In all the previous configurations (a-e), the particle (dark blue) is propelled along the waveguide in the direction of the wave vector component parallel to the interface from which the evanescent wave emanates. (f) If counter-propagating mutually coherent evanescent waves are employed in the geometries (a-e), an evanescent conveyor belt is obtained, in which particles can be transported bidirectionally [105, 272, 374-376].

of nanoparticles along a silicon waveguide patterned with periodically placed nanorods tuned to different plasmonic resonant frequencies. Periodic switching of the illuminating laser source between the respective resonant wavelengths consecutively excited resonances in nanorods placed at different spatial locations. This resulted in a time-dependent, pulsating optical force that moved the particles between adjacent nanorods in a peristaltic manner [377]. 
(a)
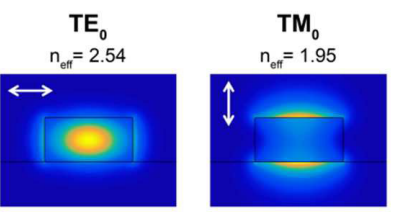

(c)

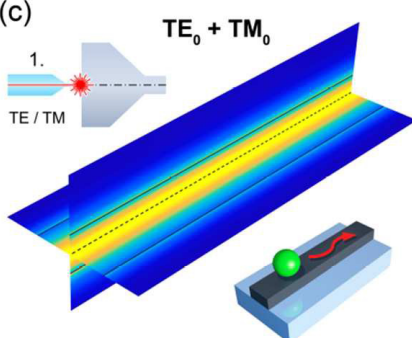

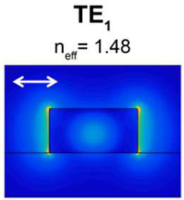

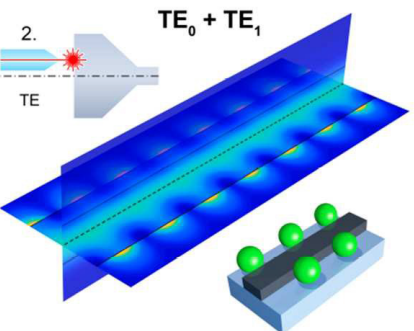

(b)
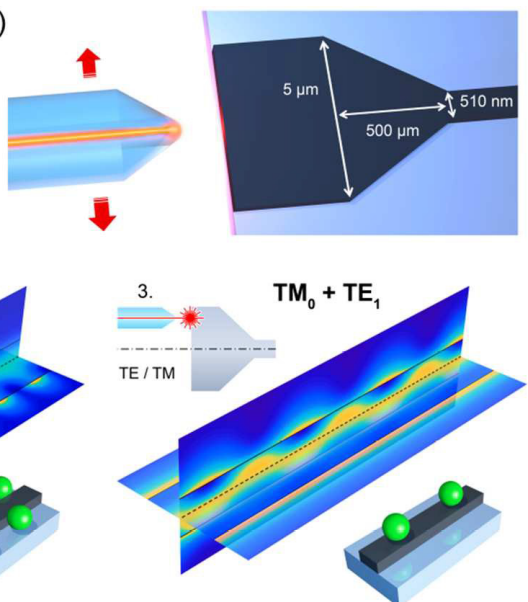

Fig. 36. Confinement and transport of micro- and nanoparticles in structured evanescent waves produced by near-field mode beating in a few-mode silicon waveguide. (a) Intensity profiles and effective indices of the three guided modes supported by a $510 \times 248 \mathrm{~nm}^{2}$ silicon waveguide at telecom wavelengths $(1530 \mathrm{~nm})$. For each mode, the white arrow indicates the direction of the electric field's dominant component. (b) Schematic representation of the used light coupling scheme. The light is coupled into the waveguide from an optical fiber with a lensed tip. (c) Horizontal and vertical cross-sections of the effective intensity distributions resulting from the respective co-propagation of the $\mathrm{TE}_{0}-\mathrm{TM}_{0}$, the $\mathrm{TE}_{0}-\mathrm{TE}_{1}$, and the $\mathrm{TM}_{0}-\mathrm{TE}_{1}$ modes along a 10- $\mu \mathrm{m}$-long portion of the waveguide. For each case, the light coupling conditions at the entrance of the waveguide are indicated: 1. centered fiber and excitation of both polarization components; 2 . slightly off-axis fiber and excitation of the horizontal polarization component only; 3. off-axis fiber and excitation of both polarization components. The behavior of particles trapped at the surface of the waveguide is also schematically depicted: 1 . particles guided on top of the waveguide; 2 . particles held in place at the sides of the waveguide; 3 . particles held in place on top of the waveguide. Adapted with permission of Royal Society of Chemistry, from [361]; permission conveyed through Copyright Clearance Center, Inc.

(a)

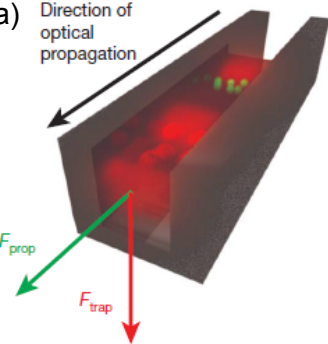

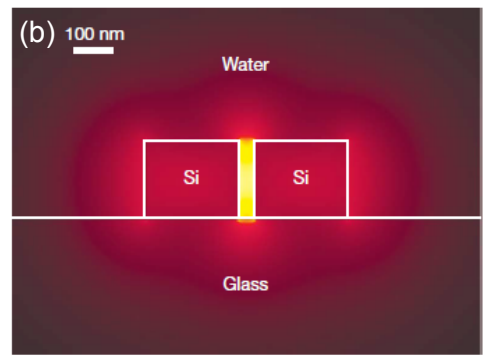

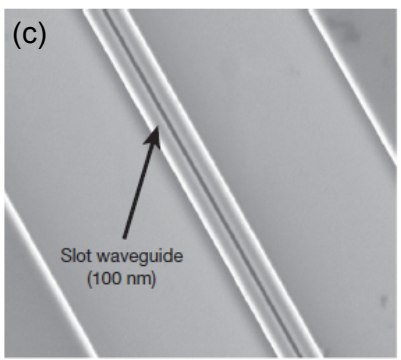

Fig. 37. Sub-wavelength slot waveguide for the transport of nanoparticles or biomolecules. (a) Illustration of transport of two different sizes of nanoparticles. $F_{\text {prop }}$ represents the radiation pressure force responsible for the transport and $F_{\text {trap }}$ denotes the gradient trapping force that holds the nanoparticles within the slot region. (b) Calculated mode profile for a silicon-on-insulator 40-nm slot waveguide immersed in water. The main trapping region is in the high-intensity slot mode, although alternate trapping positions are located on the sides of the waveguide, where two decaying evanescent modes extend into the surrounding liquid medium. (c) Scanning electron microscope image of a 100-nm slot waveguide structure. Adapted by permission from Springer Nature Customer Service Centre GmbH: Nature [368], Copyright 2009. 
(a)

(b)
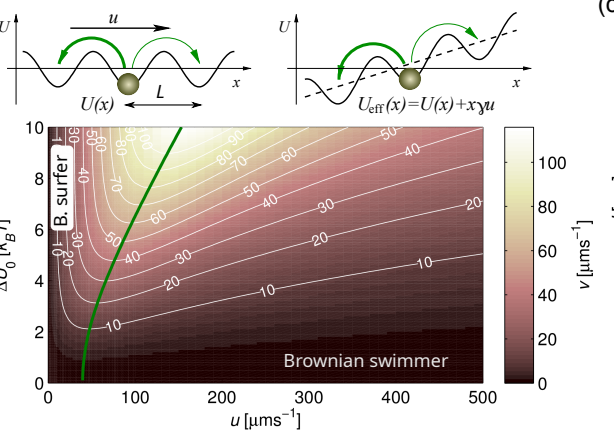

(c)

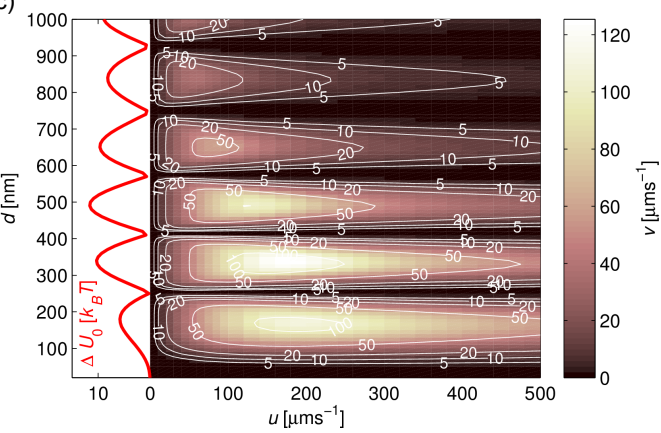

Fig. 38. Characterization of particle transport in an evanescent conveyor belt. (a) An object confined in a periodic potential moving in a viscous medium behaves identically to an object confined in a static but tilted periodic potential. (b) The mean delivery speed $v$ calculated for a polystyrene sphere of diameter $350 \mathrm{~nm}$ in water dragged by a traveling standing wave with period $L=200 \mathrm{~nm}$ and speed $u . \Delta U_{0}$ denotes the depth of the potential well of the periodic potential in (a). The green curve reveals that there exists an optimal combination of parameters providing the maximum delivery speed. The bottom right region of the plot corresponds to a Brownian swimmer while the top left region represents a Brownian surfer. The same transport principles also apply in radiative fields, as already mentioned in section 3.3.2. (c) The mean delivery speed $v$ as a function of the particle diameter $d$ and the speed of the traveling standing wave $u$ assuming $\Delta U_{0}=10 k_{\mathrm{B}} T$ for $d=350 \mathrm{~nm}$. The plot shows there exist optimal particle sizes, for which the particle delivery in the conveyor belt is enhanced or, on the contrary, fully suppressed. Reproduced from [272] by permission of IOP Publishing under CC BY-NC-SA. Copyright 2008 Deutsche Physikalische Gesellschaft.

(a)

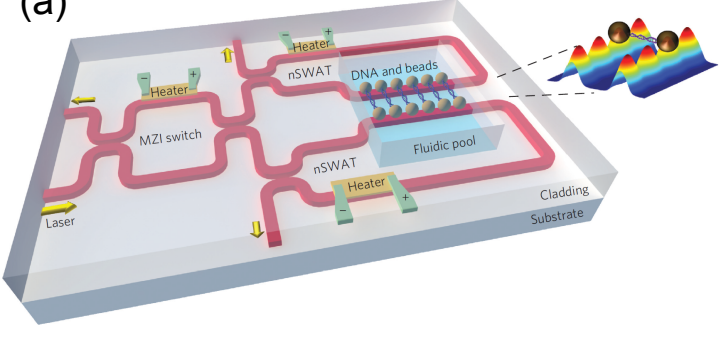

(b)

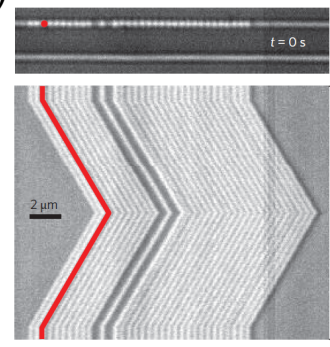

Fig. 39. Single-molecule mechanical experiments in evanescent conveyor belts formed in optofluidic chips. (a) Schematic of the optofluidic chip design. Nanophotonic standing wave array traps (nSWATs) are implemented with silicon waveguides fabricated on a silicon-oninsulator platform. The laser input to the waveguides is partitioned into two nSWATs using a Mach-Zehnder interferometer (MZI). Counter-propagating waves forming the two nSWATs are obtained with the use of 50/50 integrated beamsplitters, which have their output arms connected. Three microheaters are located above the waveguides, one at the MZI to control partitioning of the laser into the two nSWATs and two more to control the trap positions in each nSWAT. The microheaters and waveguides are buried in an oxide layer, with exception of the exposed waveguides in the fluidic pool trapping region. Inset: Array of traps (red) with a DNA molecule suspended between two beads held by nSWATs. (b) An array of beads (356 $\mathrm{nm}$ in diameter, one colored) trapped on the upper waveguide and transported in a controlled manner along it in both directions. Adapted by permission from Springer Nature Customer Service Centre GmbH: Nature Nanotechnology [376], Copyright 2014. 


\subsection{Transport based on optical fibers}

Optical fibers represent by far the most widespread class of optical waveguides. Thus, similar strategies to those discussed in Section 4.2 can be used for fiber-based particle transport. In particular, the radial intensity gradient of the evanescent field outside the fiber localizes the lateral position of the particles, while either the radiation pressure or the conveyor belt are employed to guide the particles along the fiber. Alternatively, lateral confinement of the particles can be provided within hollow-core photonic crystal fibers. Various geometries employing optical fibers for particle transport are presented in Fig. 40.
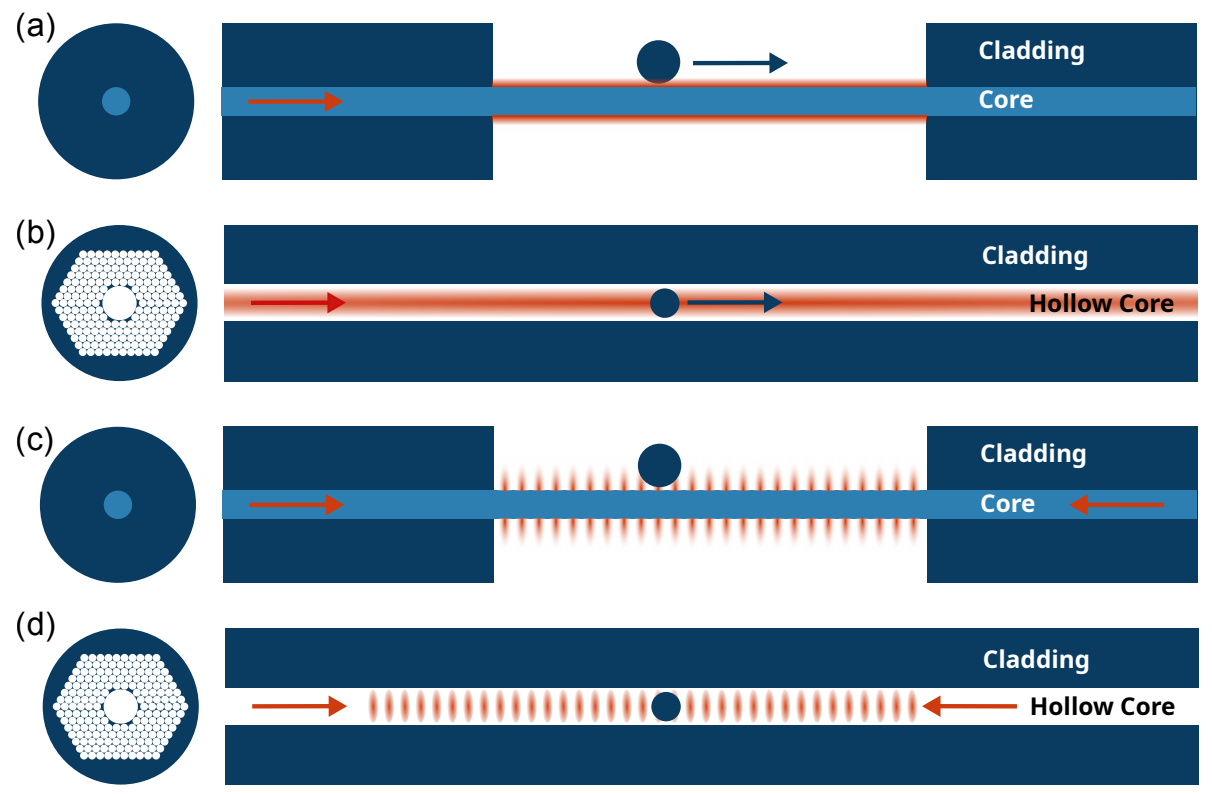

Fig. 40. Possible strategies for particle transport using optical fibers. (a) A particle is dragged by an evanescent field propagating above the surface of an exposed core of a fiber with removed cladding [378] or above a tapered fiber. (b) The radiation pressure of a high-intensity guided mode formed within a hollow-core photonic crystal fiber is used to push the particle along the fiber. (c) Fiber-based and (d) hollow-core fiber-based optical conveyor belts for bidirectional particle transport can be formed by launching two counter-propagating mutually coherent waves into the fiber.

\subsubsection{Liquid-fiber interfaces}

Step-index optical fibers represent an experimental platform for transporting objects immersed in a liquid along non-planar solid-liquid interfaces [378]. In conventional optical fibers, the guided modes are confined within the fiber core and the evanescent field of the mode resides in the solid fiber cladding; thus, neither radiative nor evanescent waves are accessible for particle transport. This, however, can be changed by tapering the fiber into a sub-wavelength diameter using thermal treatment or by the careful removal of the fiber cladding [379]. In such modified fibers, the evanescent component of the fiber mode becomes significantly more pronounced and ensures stronger interaction with nearby suspended objects with relative refractive index $m>1$, which are pulled to the fiber surface by the optical gradient force (see Fig. 40a). Such nanofibers can be easily shaped or looped into ring resonators with high $Q \simeq 10^{4}$ [380], in which the optical forces can be further enhanced. Optical binding between multiple particles self-assembled along the fiber leads to the formation of a quasi-solid structure with modified delivery speed depending on 
the guided mode excited in the fiber [381]. Focused ion beam milling can been employed to create nanostructures within the optical fiber, e.g., a slot waveguide-like region in a tapered optical fiber, at which the optical forces are greatly enhanced and nanoobjects can be localized [382].

An alternative geometry can be exploited with hollow-core photonic crystal fibers whose core is filled with a suitable liquid containing suspended particles (see Fig. 40b). Since the particles are confined by solid walls in the high-intensity region occupied by the guided mode of the fiber, they experience significantly higher scattering forces than those produced by evanescent fields. Using this geometry, suspended dielectric particles were guided over the distance of several centimeters, with velocities on the order of tens of $\mu \mathrm{m} \cdot \mathrm{s}^{-1}$ [383]. Since the various types of optical waveguides and fibers are designed to guide light over long distances, they can offer an unbeatable distance for the transport of microparticles and nanoparticles. The typical velocity of particle transport is in the range of $0.5-0.01 \mu \mathrm{m} . \mathrm{s}^{-1}$ per milliwatt of incident power, varying slightly with the particle size and optical properties. We refer the reader elsewhere for a more detailed overview on this particular topic [9,380,384-386].

\subsubsection{Gas-fiber interfaces}

In addition to particles suspended in liquids, particle transport has been demonstrated near interfaces between air and the surface of a subwavelength-diameter optical fiber; this configuration has been also exploited for manipulating and guiding cold atoms in vacuum $[387,388]$. If the distance between the particle (atom) and the fiber surface is on the order of tens of nanometers, a very strong van der Waals attraction causes adhesion of the particle to the surface. Thus, a repulsive force must be present to keep the particle sufficiently far from the surface. To this end, blue-detuned evanescent waves have been employed to repel cold atoms from the fiber surface [389]. The principle of the evanescent conveyor belt formed along a tapered fiber has been then used for delivery of cold Cs atoms over millimeter-scale distances [390].

Hollow-core optical fibers have been extensively studied for transporting cold atoms [391]; for example, they were employed to deliver atoms between two separate vacuum chambers over macroscopic distances [392]. Similar fibers were also used to trap and guide micron-sized aqueous aerosol droplets [393]. The invention of hollow-core photonic crystal fibers (HC-PCFs) with a sufficiently large cross-sectional area of the central hole [394, 395] allowed for the low-loss transport of solid and liquid particles within the air-filled modal volume [396] or for the bidirectional transport of particles using the conveyor belt principle [397,398]. Gas-filled HC-PCFs represent a new paradigm in fiber-based sensing, because they potentially allow for mapping of multiple physical quantities (e.g., pressure, viscosity, temperature, electric or magnetic field, ionizing radiation) with sub-millimeter positional accuracy over kilometer-scale distances [399] (see Fig.41 for an illustration).

\subsection{An interplay of forces of optical and non-optical nature}

Besides relying solely on the direct transfer of momentum between incident light waves and illuminated microscopic particles, as in the strategies previously discussed in Section 3 and Section 4 , gradient and scattering optical forces can be complemented by other external forces acting on the moving particles, such as gravity, hydrodynamic forces, acoustic forces, or electromagnetic forces. Such combinations of external forces of varying physical origin provide additional degrees of freedom that allow for the selective transport and separation of particles with differing material properties.

\subsubsection{Optical chromatography}

In a typical experimental configuration used in optical chromatography, particles suspended in a viscous liquid are simultaneously subjected to the radiation pressure $F_{\mathrm{rp}}$ of a moderately focused laser beam and a counteracting hydrodynamic drag force $F_{\mathrm{h}}$ due to liquid flow in the 
(a)

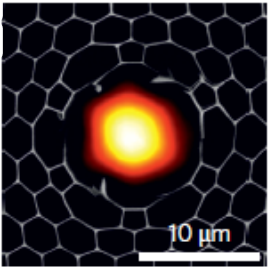

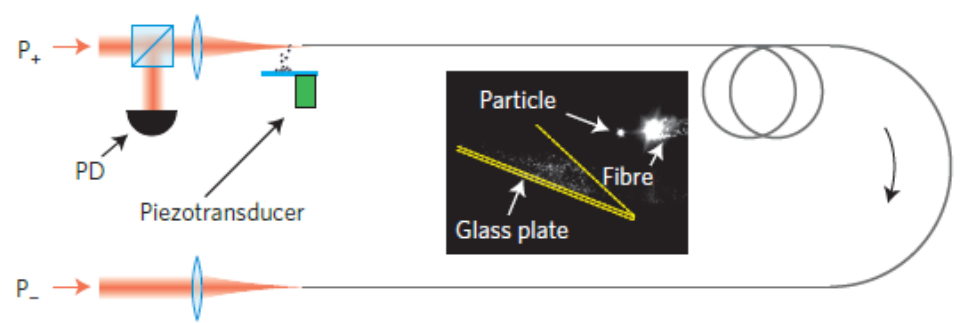

(b)

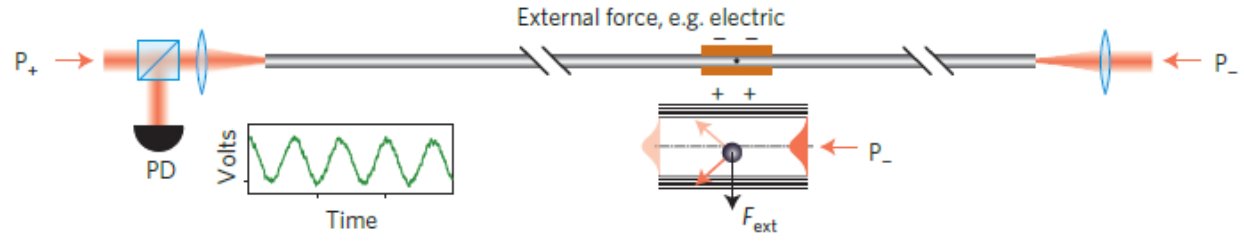

(c)

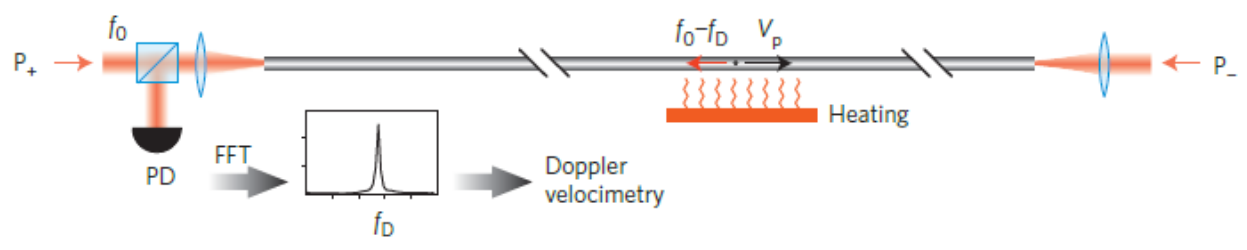

(d)

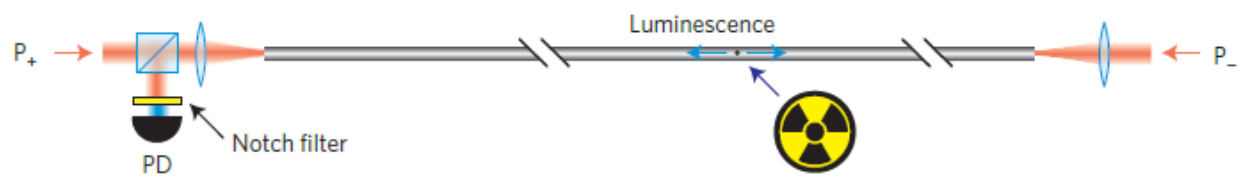

Fig. 41. Hollow-core fiber sensors using particles optically guided in air. (a) Experimental implementation of a sensor based on optically guided particles confined within a HC-PCF. The left part shows a scanning electron micrograph of a HC-PCF (core diameter: $12 \mu \mathrm{m}$ ) with a superimposed near-field optical mode profile (at $1064 \mathrm{~nm}$ wavelength). A particle is initially trapped in front of the HC-PCF. By adjusting the power in the forward and backward propagating beams $\left(P_{+}, P_{-}\right)$the particle can be moved along the fiber or held stationary. The transmitted power is monitored using a photodiode (PD). (b-d), Different types of particle-environment interactions can occur in the measurement region: (b) an external force acting on the particle, (c) a change in environmental conditions, for example, in temperature, or (d) a change in the particle state, for example, radioluminescence. Adapted by permission from Springer Nature Customer Service Centre GmbH: Nature Photonics [399], Copyright 2015.

direction opposite to the beam propagation [400] (see Fig. 42). In most practical cases, the particles of interest (colloids, supramolecular clusters, or living cells) have a refractive index higher than that of the host liquid (typically water). Hence, for an incident beam with a Gaussian transverse profile of optical intensity, the particles are attracted toward the beam axis by the radial optical gradient force given by Eq. (18) [401]. Since the incident beam is moderately focused, its intensity - and, consequently, the generated radiation pressure $F_{\mathrm{rp}}$ pushing the particles in the beam propagation direction - varies along the beam axis according to Eq. (16). On the other hand, the hydrodynamic drag $F_{\mathrm{h}}=-\gamma v_{\mathrm{h}}$, which pushes the particles with hydrodynamic drag coefficient $\gamma$ against the incoming beam, is constant, provided that the velocity $v_{\mathrm{h}}$ of the host fluid flow is uniform everywhere. The counteracting $F_{\mathrm{rp}}$ and $F_{\mathrm{h}}$ scale differently with the particle radius $a$. As a result, illuminated particles of different sizes find different equilibrium positions along the beam axis, where the net force $F_{\mathrm{rp}}+F_{\mathrm{h}}=0$ [402]. Besides the particle size, the 
radiation pressure also depends on the optical properties of the transported particles, such as their index of refraction. Thus, optical chromatography is also capable of separating particles with identical sizes, provided that they have a sufficient contrast of refractive index [403].

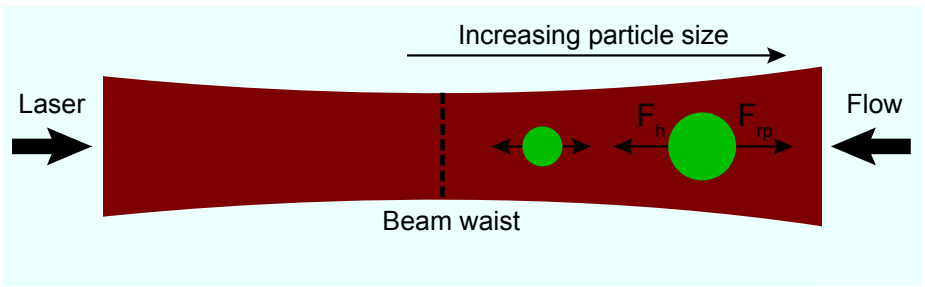

Fig. 42. Operating principle of optical chromatography. Suspended particles of various sizes and/or refractive indices are subjected to both radiation pressure $F_{\mathrm{rp}}$ and a counteracting hydrodynamic drag $F_{\mathrm{h}}$. The particles travel to different equilibrium positions where $\left|F_{\text {rp }}\right|=\left|F_{\mathrm{h}}\right|$ for each particle.

The main advantages of optical chromatography for particle sorting lie in the possibility of quickly adjusting the sorting criteria by changing the beam diameter and/or power, in the easiness of detection of the separated particles, and in the simultaneous concentration and sorting of dilute samples. The efficiency of particle fractionation can be further increased by cascading several optical chromatography regions with different parameters of the incident beam [15]. Using the above experimental procedure, optical chromatography has been successfully applied for discerning micron-sized polystyrene beads differing as little as $70 \mathrm{~nm}$ in diameter [404], for quantifying the optical forces acting on hybrid dielectric-metal core-shell particles [405], for the real-time monitoring of immunological reactions between antibody-coated beads and antigen suspended in the host medium [406,407], and for the fractionation of blood cells [408]. We refer the reader to review [21] for additional examples of applications of optical chromatography.

Most optical chromatography implementations use a laser beam propagating either through a capillary $[400,405,406]$ or through a specially designed flow cell $[15,403,408]$ fabricated from fused silica. This material with excellent optical transmission properties in the visible and near-infrared parts of the spectrum is chosen to reduce absorption and scattering of the incident laser light from the device walls. Even though this configuration is relatively simple, alignment of the free-space beam with the flow channel axis represents a challenge in quantitative optical chromatography experiments, which indeed rely on the precisely characterized balance of optical and hydrodynamic forces. Alternatively, the light for actuating the particles in the channel can be delivered through a photonic crystal fiber [409]. With this approach, the actuation laser beam is always intrinsically aligned with the channel. Since the light does not have to propagate through the device walls, these can be made from polydimethylsiloxane (PDMS) by conventional soft-lithography, which greatly enhances the flexibility in designing the layout of the experimental device. Moreover, the input photonic crystal fiber can serve a dual purpose by simultaneously delivering both the manipulation light and the light for exciting fluorescence in the target particles for visualization and detection purposes. Using photonic crystal fiber-based optical chromatography setups, size-driven and refractive index-driven separation of binary mixtures of colloids and separation of cell populations with/without internalized fluorescent marker beads were demonstrated (see Fig. 43).

Instead of using a laser beam propagating against the direction of the fluid flow, an alternative geometry can be employed, in which the beam propagates perpendicularly to the flow. In this so-called cross-type optical chromatography, proposed independently in [410] and [411], variations of radiation pressure acting on particles of different sizes and/or materials result in these particles being pushed into different flow streamlines during the time period of their 
(a)
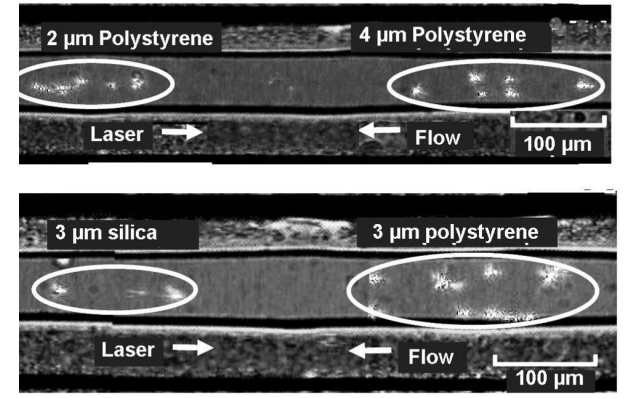

(b)

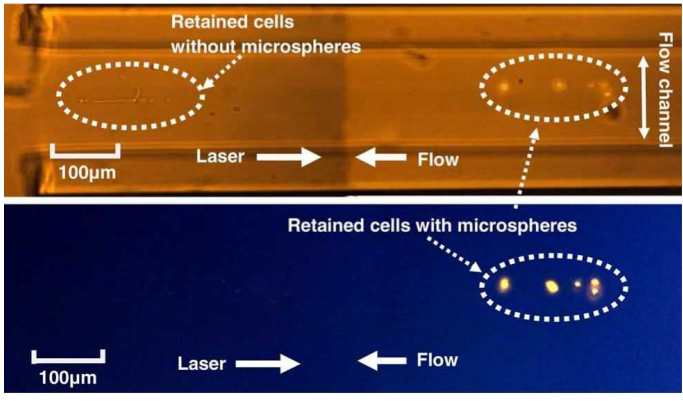

Fig. 43. Particle separation using optical chromatography based on photonic crystal fibers. (a) Size-driven separation of a binary mixture of polystyrene particles containing spheres of diameters of $4 \mu \mathrm{m}$ and $2 \mu \mathrm{m}$ (top). Refractive index-driven separation of a binary mixture of polystyrene particles and silica particles of diameter of $3 \mu \mathrm{m}$ (bottom). (b) Separation of HEK cells incubated with red fluorescent polystyrene microspheres, visualized (top) with simultaneous bright field illumination and fluorescence excitation, and (bottom) with fluorescence excitation only. Adapted with permission from [409]. Copyright 2010 Optical Society of America.

interaction with the beam. Under laminar flow conditions, the particles remain confined in their respective streamlines upon leaving the illuminated region; thus, they are effectively separated across the flow. Because the flow continuously removes the particles from the separation region, cross-type optical chromatography in principle allows for easier particle transport and much larger separation throughputs.

\subsubsection{Transport of particles using the Magnus effect}

The Magnus effect is a phenomenon that is commonly associated with the motion of spinning objects through a fluid. In particular, the path of the spinning object through the fluid is deflected in a manner that is not observed when the object is not spinning. The deflection can be explained by the difference in the fluid pressure on opposite sides of the spinning object. This effect is well known at the macroscopic scale: for example, it is commonly observed in various sports when the trajectory of a spinning ball is curved or it provides propulsive forces for a ship equipped with a Flettner rotor. However, only a few articles have dealt with the characterization of this phenomenon at the microscopic scale, where fluid flows are described by low Reynolds numbers. If a spherical particle of radius $a$ immersed in a fluid with density $\rho$ moves through the fluid with a velocity $\mathbf{v}$ and, in addition, the particle spins with angular velocity $\boldsymbol{\Omega}$, one can obtain the force acting on the particle from the Navier-Stokes equations [412,413]:

$$
\mathbf{F}_{\mathrm{M}}=\pi a^{3} \rho \mathbf{\Omega} \times \mathbf{v}[1+O(\mathrm{Re})]
$$

where $\operatorname{Re}=\rho|\mathbf{v}| a / \eta$ denotes the Reynolds number and $\eta$ is the viscosity of the fluid. For a long time it was believed that, for a small sphere in a slow shear flow, the Magnus force can be neglected with respect to the viscous drag acting on the particle [414]. However, recent experimental observations [413] have indicated that this effect is observable and probably could be even enhanced for fast spinning microobjects [415]. Figure 44 shows the configuration of the experiments reported in [413]. Two circularly polarized co-propagating beams intersecting at an angle interfere and create fringes (see Fig. 26b), in which a birefringent liquid crystal droplet is trapped. Due to the transfer of angular momentum from the circularly polarized light beams to the birefringent droplet, the droplet is also spinning. The periodic modulation of the fringe position results in dragging the spinning particle along, similarly to the optical conveyor belt 
described in Section 3.3. Following Fig. 44a, if the droplet spins counter-clockwise and the moving fringe drags it along the positive $x$-axis (to the right), the Magnus force $\mathbf{F}_{\mathrm{M}}$ given by Eq. (46) pushes the droplet upwards, along the positive $y$-axis. Along the $x$-axis, the viscous drag force acting on the moving droplet is compensated by the gradient optical force coming from the fringe intensity profile. Consequently, the droplet is horizontally confined in an equilibrium position located slightly to the left from the fringe intensity maximum. Along the $y$-axis, however, the optical gradient force only becomes significant when the droplet is located in the proximity of the top edge of the fringe. Thus, the droplet moves upwards along the fringe (the positive $y$-direction) due to the uncompensated Magnus force. Upon reversing the direction of the fringe motion, the Magnus force starts pointing downwards, pushing the droplet in that direction, and the situation mirrors the behavior observed in the first half-period of the modulation cycle. The motion of the spinning droplet during the fringe modulation cycle is shown in Fig. 44b-e for different senses of the droplet rotation.

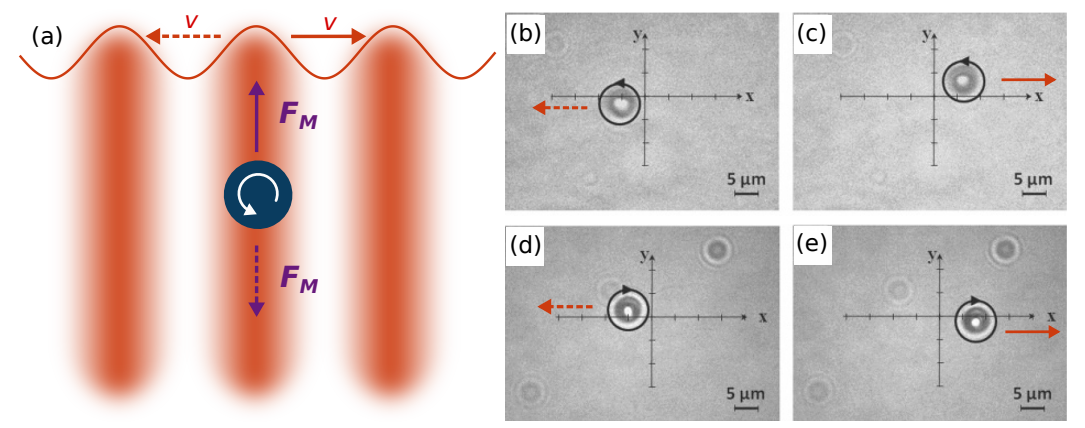

Fig. 44. Micro-scale demonstration of the Magnus effect. (a) A birefringent liquid crystal droplet is trapped in an interference fringe moving horizontally with a velocity $\mathbf{v}$ and is forced to rotate with an angular velocity $\boldsymbol{\Omega}$ due to the transfer of angular momentum from the circularly-polarized incident light to the droplet. Depending on the sense of the droplet rotation and the direction of its translation, the droplet moves upwards or downwards due to the Magnus force $F_{\mathrm{M}}$. For a counter-clockwise rotating droplet, $F_{\mathrm{M}}$ pushes the droplet (b) downwards for the fringe moving to the left and (c) upwards for the fringe moving to the right. When the sense of the droplet rotation is reversed, the vertical motion of the droplet in the corresponding phases of the fringe modulation cycle reverses direction, as can be seen by comparing (b) with (d) and (c) with (e). Adapted with permission from [413]. Copyright 2011 by the American Physical Society.

According to Eq. (46), a larger microparticle spinning fast in a liquid or air flow should feel an even stronger Magnus force. Thus, in principle, the Magnus effect could be used for particle transport. However, this has not been experimentally investigated in depth yet. 


\subsection{Transport with plasmonic fields}

The possibility of spatial confinement of micro- and nano-objects with plasmonic force fields has been extensively reviewed elsewhere $[12,49]$. The majority of plasmonic micromanipulation schemes have been developed to localize tiny particles - down to the level of individual supramolecular complexes and macromolecules - near metal-dielectric interfaces. At such interfaces, the local electric field can be largely enhanced by exciting collective charge oscillations, which can have the form of either traveling surface plasmon polaritons (SPPs) at extended metal-dielectric interfaces or of localized surface plasmons (LSPs) at the surface of metallic nanoparticles. Such enhanced, spatially confined electric fields are associated with large gradient forces that can effectively immobilize nanoparticles placed in them. The field of plasmonic trapping is currently rather active. For example, Fig. 45 shows an exciting recent example of plasmonic nanotweezers [98]. This device is based on a bowtie nanoaperture manufactured at the tip of a metal-coated tapered optical fiber, which is mechanically positioned in the proximity of the manipulated nanoparticle. The particle can then be transported by simply moving the fiber tip. The trapping uses self-induced back-action [12], which dramatically lowers the power required to keep the dielectric nanoparticle inside a metallic nanohole, thus minimizing photothermal issues. For completeness, let us note that a similar effect of self-induced back-action was also observed with $500 \mathrm{~nm}$ dielectric particle in a dielectric 2-D photonic crystal hollow cavity [416].
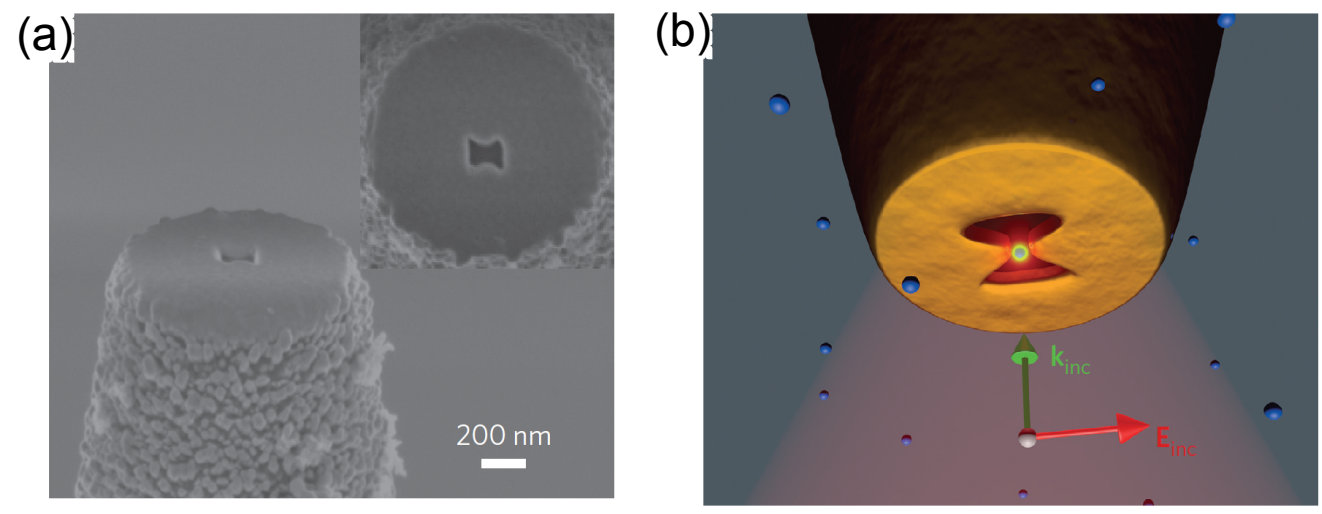

Fig. 45. Plasmonic nanotweezers formed at the front face of a mechanically positioned fiber tip. (a) Scanning electron microscopy image of an 85-nm-gap bowtie nano-aperture fabricated at the metal-coated tip of a tapered optical fiber. (b) Schematics of the experimental configuration. The nano-aperture is illuminated from the liquid side with a $1064 \mathrm{~nm}$ laser beam focused through an objective of numerical aperture 0.65 . The incident beam propagates along the direction of $\mathbf{k}_{\mathrm{inc}}$ and its electric field $\mathbf{E}_{\mathrm{inc}}$ is aligned along the nano-aperture gap to excite its transverse resonant mode. Adapted by permission from Springer Nature Customer Service Centre GmbH: Nature Nanotechnology [98], Copyright 2014.

With the use of plasmonic fields, it is possible to generate optical pulling forces similar to those observed in the tractor beams discussed in Section 3.2. Figure 46 illustrates a simplified model situation of a spherical dielectric nanoparticle placed in the vicinity of a metal surface and illuminated by an incident wave. In describing the behavior of the nanoparticle, the retarded field induced by the point dipole representing the nanoparticle and subsequently reflected from the surface must be taken into account. In the Rayleigh approximation, the contribution $F_{x}$ to the net force coming from the optical coupling of the nanoparticle with the surface can be described as [417]:

$$
F_{x}=-\frac{k^{2}}{\varepsilon_{0}} \mathfrak{I}\left\{d_{x}^{*} d_{z}\right\} \mathfrak{I}\left\{\partial_{x} G_{x z}^{R}\right\}
$$


where $\mathbf{d}$ and $\mathbf{G}^{\mathbf{R}}$ denote the induced dipole moment of the particle and the dyadic Green's function of the dipole-interface system [119]. For a dipole sufficiently close to the surface, it holds that

$$
\begin{aligned}
\mathfrak{I}\left\{d_{x}^{*} d_{z}\right\} & =\left|\alpha_{0}\right|^{2}\left|E_{0}\right|^{2} \sin (2 \theta) \mathfrak{I}\left\{r_{\mathrm{p}}(\theta)\right\}, \\
\mathfrak{I}\left\{\partial_{x} G_{x z}^{R}\right\} & =\frac{1}{8 \pi k^{2}} \mathfrak{I}\left\{\int_{0}^{\infty} k_{\|}^{3} r_{\mathrm{p}}\left(k_{\|}\right) \exp \left(2 *+\mathrm{i} k_{z} z_{0}\right) d k_{\|}\right\} .
\end{aligned}
$$

When evaluated near the surface plasmon resonance, where $\mathfrak{R}\left\{\varepsilon_{\mathrm{m}}+\varepsilon_{\mathrm{s}}\right\} \sim 0$, for a low absorbing surface with $\mathfrak{R}\left\{\varepsilon_{\mathrm{s}}\right\}<0$ and far from configurational resonance, Eq. (47) can be expressed as

$$
F_{x} \sim-\frac{k_{\mathrm{SPP}}^{7}}{8 \varepsilon_{0} k^{3}}\left|\alpha_{0}\right|^{2}\left|E_{0}\right|^{2} \sin (2 \theta) \sin \left[2\left(k_{z} z_{0}+\Phi\right)\right] \exp \left(-2 \sqrt{k_{\mathrm{SPP}}^{2}-k^{2}}\right),
$$

where $k, k_{\|}$, and $k_{z}$ denote the wavenumber, the parallel component and the normal component of the wave vector relative to the interface, respectively, and $k_{\mathrm{SPP}}=k \sqrt{\varepsilon_{\mathrm{m}} \varepsilon_{\mathrm{s}} /\left(\varepsilon_{\mathrm{m}}+\varepsilon_{\mathrm{S}}\right)}$ is the surface plasmon polariton wavenumber. As can be seen from Fig. 46, the pulling force acting on the nanoparticle is an order of magnitude stronger than the pushing force, despite the fact that the nanoparticle is represented by an induced dipole, which scatters the incident light symmetrically in the forward and backward direction. Assuming a metallic surface and a perpendicularly incident Gaussian beam, theoretical predictions indicate that, depending on the position of the beam focus above or below the metal surface, the dielectric nanoparticle can be either trapped at the beam axis or repelled from the beam axis [418]. Near a metallic surface supporting SPPs, the emergence of pulling and pushing forces has also been theoretically predicted for larger particles supporting whispering gallery modes $[419,420]$.

Particle transport relying on LSPs has also been demonstrated . For example, a complex polarization-sensitive metasurface lens was realized to achieve longitudinal particle transport in a fluid. This lens was based on plasmonic nano-antennas organized within its overlapping Fresnel zones and embedded in the fluid. As it was experimentally demonstrated, this lens provided several stable trapping positions along the optical axis depending on the polarization of the perpendicularly incident beam. Thus, an illuminated particle could be moved longitudinally in a controlled way just by rotating the polarization of the incident light [421,422] (see Fig. 47). By tailoring the illumination pattern of the incident light beam with a computer-controlled spatial light modulator, constructive and destructive interference of plasmon waves was used to create a focused hotspot that could be moved across the surface. Following the hotspot position, $200 \mathrm{~nm}$ diameter nanoparticles were moved along arbitrary patterns, for example, in circular motion with linearly-polarized illumination light [423].

Illumination of transported metallic objects or constraining nanostructures with light wavelengths near their plasmon resonances is inevitably associated with generation of heat that has to be dissipated to the surrounding liquid; otherwise, the objects can be destroyed or the forces arising from temperature gradients become dominant over the optical forces. Utilization of such mechanisms for particle delivery is reviewed in the following sections. 

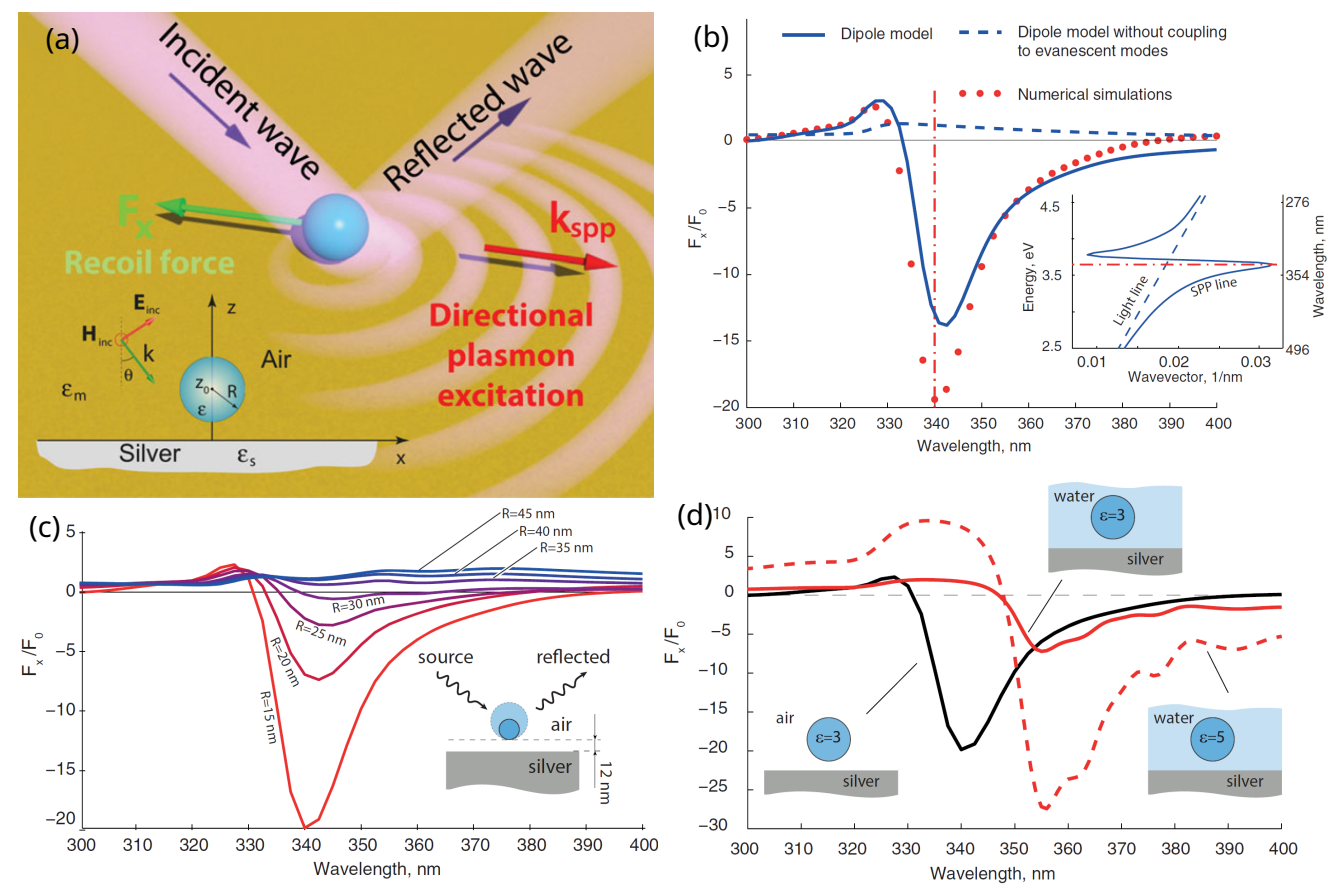

Fig. 46. Emergence of optical pulling forces for a nanoparticle optically coupled to a plasmonic surface. (a) Directional excitation of surface plasmon polaritons by a small particle placed in the vicinity of a metal surface. The recoil force created by the plasmon polaritons acting on the particle can be directed opposite to the propagation direction of the incident light. The inset depicts the geometry of the problem. (b) Theoretical spectral dependence of the longitudinal force $F_{X}$ acting on a dielectric particle near the plasmonic surface in air, normalized with respect to the radiation pressure $F_{0}$ acting in the absence of the surface. Calculation parameters: particle radius $R=15 \mathrm{~nm}$, particle dielectric permittivity $\varepsilon$ $=3$, distance from a silver surface $z_{0}=27 \mathrm{~nm}$, angle of incidence of the incident wave $\theta$ $=35^{\circ}$. The solid and dotted curves correspond to the approximate dipole model and full numerical calculations, respectively. The dashed curve corresponds to the dipole model with excluded coupling to the evanescent modes. Inset: the dispersion curve of the SPP at the silver-air interface. The red dash-dotted line corresponds to the SP resonance wavelength. (c) Theoretical spectral dependences of $F_{X}$ for different particle radii $R$ in air with a constant distance of the particle from the surface. The blue curves correspond to the absence of coupling to the evanescent modes. (d) Theoretical spectral dependences of $F_{X}$ for a particle with radius $R=15 \mathrm{~nm}$, whose center is separated $27 \mathrm{~nm}$ from the surface immersed in different environments. Also in this case, $F_{X}$ is normalized with respect to $F_{0}$. Adapted with permission from [417]. Copyright 2016 John Wiley and Sons. 

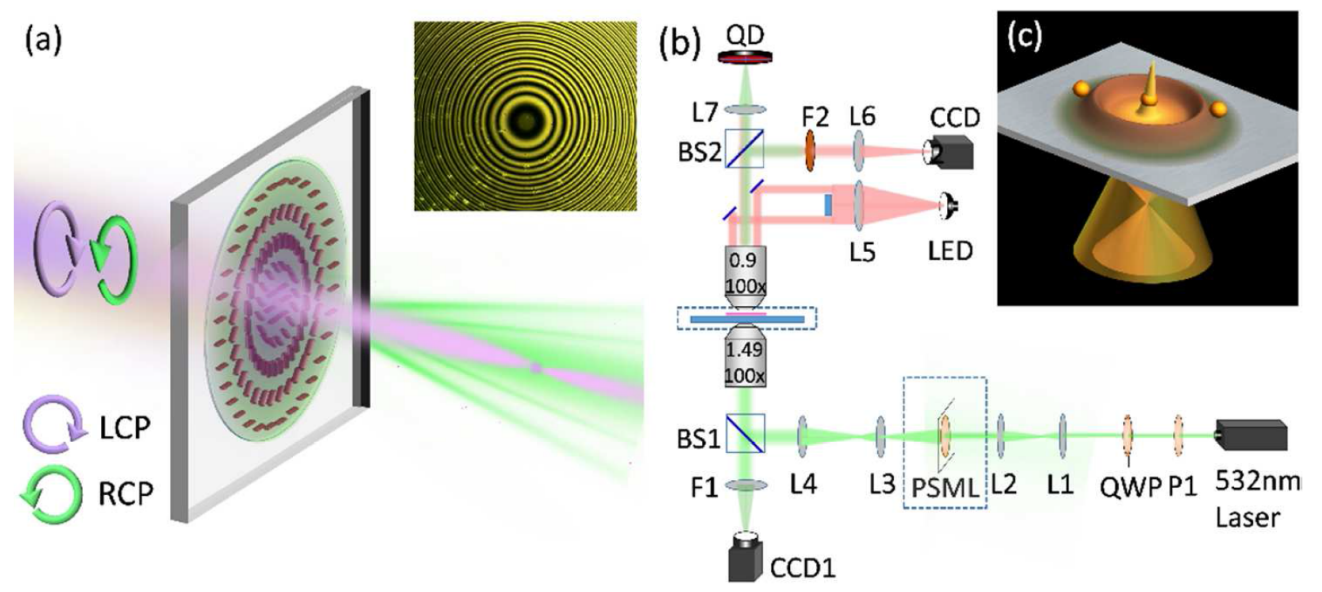

Fig. 47. Particle transport with the use of polarization-sensitive metasurface lenses. (a) Schematics of a polarization-dependent planar metalens with its focal plane located at different longitudinal positions depending on whether the incident beam is right-hand (RCP) or left-hand (LCP) circularly polarized. (b) An experimental setup forming the tailored plasmonic trapping system using the polarization-sensitive metalens. (c) An example of particle trapping using the experimental system shown in (b). Adapted with permission from [422] Adapted with permission from [422]. Copyright 2018 American Chemical Society. 


\section{Light-induced phoresis in non-optical fields}

In all the previously discussed strategies for light-induced transport of micro- and nanoparticles, spatial confinement and/or motion of the illuminated particles was, at least in part, the result of the direct transfer of momentum carried by the incident photons. The energy of light can also be harnessed to transport particles through indirect mechanisms, where light acts as a precisely controlled switch for modulating non-optical fields resulting, for example, from gradients of electric potential, $\mathrm{pH}$, concentration, or temperature. The main advantage of such an approach lies in the possibility of generating large forces that subsequently initiate directional motion of colloidal particles, referred to as phoresis, without the necessity of applying high-intensity laser beams that can be detrimental to the target sample. To first approximation, we can identify two broad classes of light-induced phoretic transport. In a first type of approach, light is used to form external gradients inducing motion of particles directly in their surroundings. In this section, we will explore two well-established examples of this type of strategy: optically induced dielectrophoresis in an electric field (Section 5.1) and thermophoresis in a temperature gradient (Section 5.2). Alternatively, light can be used to create an asymmetry in the physical or chemical properties of the illuminated particles (e.g., in their temperature profile, size and shape, or surface chemistry). As a result of these modifications, the particles can then undergo self-phoresis powered by energy exchange with the environment through some form of free-energy conversion. Below, we will discuss in detail different mechanisms of such light-induced transport. In particular, we will discuss photophoresis in absorbing particles (Section 5.3), enhanced diffusion in aqueous environments (Section 5.4), propulsion via material deformation (Section 5.5), and transport driven by capillary forces and Marangoni stresses (Section 5.6).

\subsection{Optically induced dielectrophoresis}

(a)

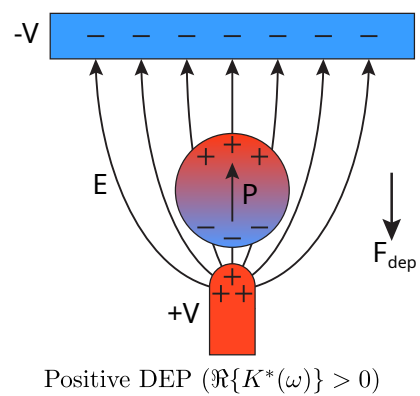

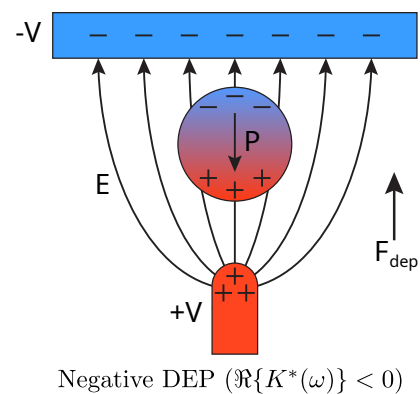

(b)

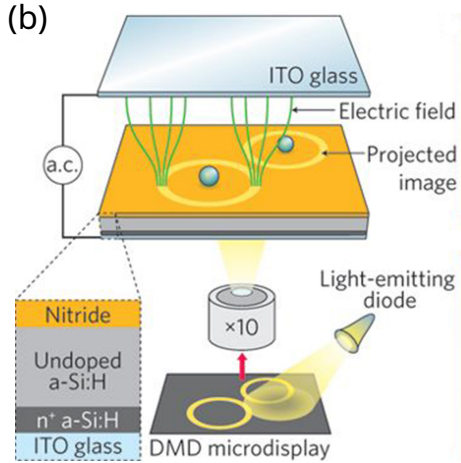

Fig. 48. Manipulation of electrically neutral dielectric particles by optically induced dielectrophoresis (DEP). (a) An inhomogeneous electric field $\mathbf{E}$ induces a dipole moment $\mathbf{P}$ in the particle. Depending on the value of the Clausius-Mossotti factor $K^{*}(\omega), \mathbf{P}$ is either parallel to $\mathbf{E}$ and the particle moves to the high-field region (positive DEP) or anti-parallel to $\mathbf{E}$ and the particle moves to the low-field region (negative DEP) due to the dielectrophoretic force $\mathbf{F}_{\text {dep }}$. (b) Experimental set-up of optoelectronic tweezers. Suspended particles are sandwiched between a transparent electrode and a photoconductive (amorphous silicon, a-Si:H) electrode. Light patterns from a digital projector are imaged by an objective $(\times 10)$ onto the device. The image, together with an alternating current (a.c.) electrical bias, generates "virtual electrodes" that in turn create dielectrophoretic traps in the illuminated areas. DMD - digital micromirror device; ITO - indium tin oxide. Adapted with permission from [22]. Adapted by permission from Springer Nature Customer Service Centre GmbH: Nature Photonics [22], Copyright 2011. 
Optically induced dielectrophoresis (DEP), also known as optoelectronic tweezers (OET) [22], is a prominent example of hybrid strategies for particle manipulation. Dielectrophoresis refers to the migration of electrically neutral dielectric particles placed into a spatially inhomogeneous electric field [424,425]. Formally, the origin of the dielectrophoretic force $\mathbf{F}_{\text {dep }}$ is analogous to the optical gradient force $\mathbf{F}_{\text {gr }}$ given by Eq. (11). When placed into an external electric field $\mathbf{E}$ generated between electrodes held at different electric potentials $\pm V$, a particle will polarize. To a first approximation, the polarization state of the particle can be described by an induced electric dipole moment $\mathbf{P}$. Interaction of this dipole with an inhomogeneous alternating electric field of root-mean-square magnitude $E_{\mathrm{rms}}$ and angular frequency $\omega$ then leads to the time-averaged dielectrophoretic force $\left\langle\mathbf{F}_{\text {dep }}(t)\right\rangle$ given by [425]:

$$
\begin{aligned}
\left\langle\mathbf{F}_{\mathrm{dep}}(t)\right\rangle & =2 \pi a^{3} \varepsilon_{\mathrm{m}} \mathfrak{R}\left\{K^{*}(\omega)\right\} \nabla E_{\mathrm{rms}}^{2}, \\
K^{*}(\omega) & =\frac{\varepsilon_{\mathrm{p}}^{*}(\omega)-\varepsilon_{\mathrm{m}}^{*}(\omega)}{\varepsilon_{\mathrm{p}}^{*}(\omega)+2 \varepsilon_{\mathrm{m}}^{*}(\omega)} ; \quad \varepsilon_{\mathrm{p}}^{*}(\omega)=\varepsilon_{\mathrm{p}}-\mathrm{i} \frac{\sigma_{\mathrm{p}}}{\omega} ; \varepsilon_{\mathrm{m}}^{*}(\omega)=\varepsilon_{\mathrm{m}}-\mathrm{i} \frac{\sigma_{\mathrm{m}}}{\omega},
\end{aligned}
$$

where $a$ is the particle radius, $\varepsilon_{\mathrm{m}}$ and $\varepsilon_{\mathrm{p}}$ are the respective permittivities of the surrounding medium and the particle, $\sigma_{\mathrm{m}}$ and $\sigma_{\mathrm{p}}$ are their conductivities, and $K^{*}(\omega)$ is the frequencydependent Clausius-Mossotti (CM) factor. The CM factor represents the dielectric signature of the particle; it depends on the particle size, material composition, structure, and surface charges. The sign of $\mathfrak{R}\left\{K^{*}(\omega)\right\}$ then determines whether the particle will move to the high-field region $\left(\mathfrak{R}\left\{K^{*}(\omega)\right\}>0\right.$; positive DEP) or to the low-field region $\left(\mathfrak{R}\left\{K^{*}(\omega)\right\}<0\right.$; negative DEP, as illustrated in Fig. 48a), in complete analogy to the behavior of high- and low-refractive-index particles exposed to a gradient of optical intensity. Since the sign of $\mathfrak{R}\left\{K^{*}(\omega)\right\}$ depends on the operating frequency, the direction of $\mathbf{F}_{\mathrm{dep}}$ acting on a given particle can be reversed simply by changing the value of $\omega$.

Instead of using fixed, static electrodes for generating the electric field, it is possible to exploit "virtual electrodes" produced in a photosensitive substrate illuminated with a suitably shaped light pattern, a principle that has been used for decades in xerography [428]. A clear advantage of such an approach is its dynamic nature: by changing the illumination pattern, the electrodes can be readily reconfigured. Consequently, the particles confined due to positive or negative DEP can be moved in space, following the changes in the distribution of the electric field. In its pioneering realization [429], OET was first demonstrated using optical images projected on a photoconductive layer of amorphous silicon $(\mathrm{a}-\mathrm{Si}: \mathrm{H})$ for creating high-resolution virtual electrode patterns for DEP-based manipulation. An illustration of the experimental setup used in [429] is shown in Fig. 48b. With an incident optical intensity on the order of $10 \mathrm{nW} . \mu \mathrm{m}^{-2}$ (100,000 times lower than that used in optical tweezers), this OET was able to confine and manipulate individual micron-sized polystyrene particles and live cells in a massively parallel way, creating simultaneously up to 15,000 DEP traps across the field of view. Moreover, it was possible to separate live and dead cells based on differences in their dielectric properties by choosing the operating frequency so that the live cells experienced positive DEP whereas the dead cells experienced negative DEP.

Particle confinement and transport with OET requires only low-intensity incoherent light. Consequently, heating issues frequently associated with tightly focused laser beams needed for single-beam optical trapping can be largely eliminated. Thus, OET represents a non-invasive experimental tool suitable for manipulation of delicate samples. Since its inception, OET has been used in a number of diverse applications involving real-time interactive manipulation of particles with sizes ranging from the molecular scale to tens of micrometers [430,431]. The particles successfully manipulated so far include polymer beads $[429,432]$, solder alloy microspheres [433], emulsion microdroplets [434], semiconducting and metallic nanowires [427], carbon nanotubes [435], DNA molecules [436], as well as living cells [429, 432, 437, 438]. Figure 49 provides several examples of OET manipulation of particles with dimensions spanning 
(a)

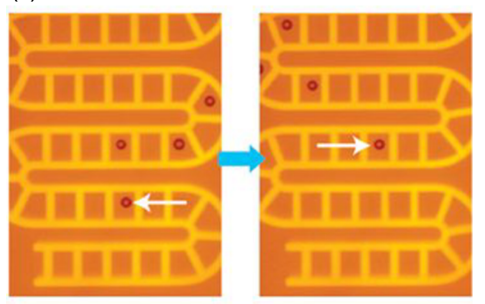

(b)

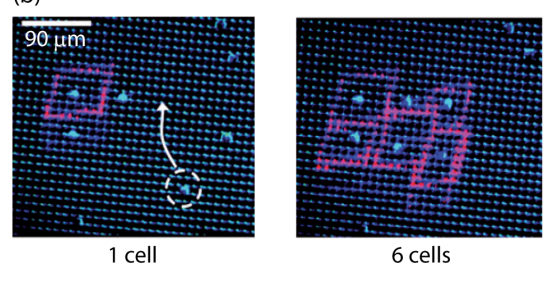

(c)
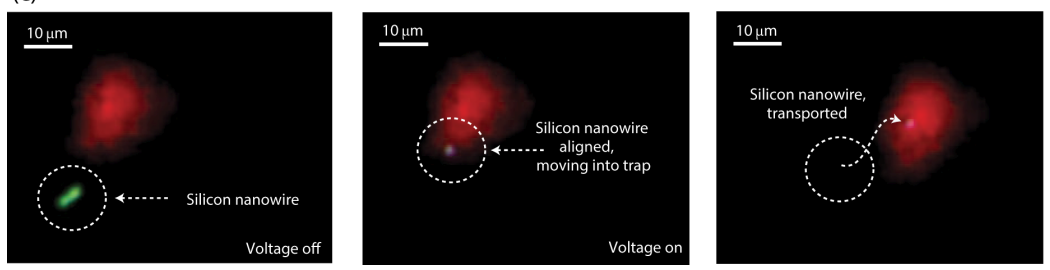

Fig. 49. Examples of particle manipulation using optoelectronic tweezers. (a) An optical conveyor belt carrying 20- $\mu$ m-diameter polystyrene particles. Adapted by permission from Springer Nature Customer Service Centre GmbH: Nature Photonics [22], Copyright 2011. (b) Patterning of individual specific HeLa cells suspended in cell culture media using a phototransistor-based OET. Adapted with permission of Royal Society of Chemistry, from [426]; permission conveyed through Copyright Clearance Center, Inc. c) Different stages of trapping of an individual silicon nanowire. The red spot indicates an illuminating $\mathrm{He}-\mathrm{Ne}$ laser beam. Adapted by permission from Springer Nature Customer Service Centre GmbH: Nature Photonics [427], Copyright 2008.

three orders of magnitude. Depending on the operating conditions - in particular, the frequency of the applied AC electric field, intensity of the illumination light, and particle size / material - various physical effects (light-induced DEP, electrothermal flow, and AC electroosmosis) contribute to the overall performance of an OET system. The relative importance of these different mechanisms has been quantitatively analyzed in [439]. The spatial resolution of optically-induced DEP traps corresponds to the size of the illuminating light spot - typically units of micrometers - and it is worse than the sub-micron-scale resolution achievable with optical tweezers. On the other hand, the OET stiffness is several hundred times higher in comparison to that of optical tweezers created with the same incident laser power [440].

In the high-conductivity physiological buffers required for live cell experiments, the maximal electric field that can be applied to the sample with conventional OET is restricted by the resistance of the a-Si:H layer forming the photoconductive electrode. Thus, manipulation of cells has to be carried out using osmotically equivalent amounts of non-electrolytes instead, which has a negative impact on cell viability. In order to overcome this limitation, a phototransistor-based OET has been proposed and implemented [425,426] (see Fig. 49b). The highly conductive phototransistors integrated into the electrode system allow generating sufficiently large potential differences between the DEP electrodes even in media with high ionic strengths, thus facilitating the manipulation of cells under proper physiological conditions.

When operating in crowded environments, it is often challenging to selectively target a single object of interest within a large population. This issue has been recently addressed in [441], where the so-called patterned OET was introduced. In this variant of an OET, conventional and optically induced DEP traps are combined in a single device. The patterns fabricated in the 
photoconductive layer form permanent DEP traps that enable continuous trapping of particles and cells even after the light source is turned off. This feature not only allows keeping the target particles immobilized for long periods of time but it also allows pushing away unwanted particles.

Optically-induced DEP traps allow for creating extended potential energy landscapes that can be readily reconfigured and optimized for largely parallel confinement and manipulation of particles with different sizes, shapes, and materials. Thus, OETs can provide a cost-effective means for flexible on-demand patterning and fabrication of colloidal micro- and nanostructures [442]. The range of future applications of OETs can be further expanded by deepening the theoretical understanding of the physical phenomena involved in optically-induced DEP, by exploring alternative photoconductive substrates for creating virtual electrodes (e.g., organic polymer coatings or photorefractive crystals), by devising electrode configurations that allow full 3-D control of the particles' position, or by combining the DEP forces with other mechanisms for particle actuation (e.g., optically induced electrowetting) [11, 22, 430, 431,442].

\subsection{Optically induced thermophoresis}

Spatially localized heating of liquids by a focused laser beam of a suitable wavelength can lead to the generation of temperature gradients that can subsequently direct the motion of particles immersed in them. Here we will discuss the actuation of particles by thermophoresis and thermal convection.

Thermophoresis [147] - and its molecular counterpart known as thermal diffusion or LudwigSoret effect - dominates in thin liquid cells where bulk convection cannot fully develop due to viscous stresses [443-445]. Thermophoretic motion of particles subjected to temperature gradients in liquids is rather complex and depends sensitively on the detailed microscopic nature of the particle/solvent interface [147]. As argued in [444], for charged particles suspended in aqueous solutions of electrolytes, the physical origin of thermophoresis lies in the entropic effects associated with the ionic shielding and hydration of the particle surface. The entropy of hydration decreases with an increasing mean temperature of the sample and can eventually change sign. Hence, both positive and negative thermophoresis, with the particles moving respectively towards or away from the heated regions, can be observed in the same particle-liquid system kept at different mean temperatures (Fig. 50a) [444]. In addition to thermophoresis, temperature gradients can also initiate convective flows that can drag the immersed particles according to Stoke's law [147]. In the most general case, both effects are present simultaneously and influence the transport dynamics of the immersed particles. A careful choice of experimental conditions with thermophoresis and liquid convection acting in opposite directions - can eventually lead to particle aggregation at locations where the two driving forces balance each other. This effect was used to realize optothermal traps for concentrating nanoparticles, such as DNA molecules [446-448], as well as microscopic colloids [449-453]. Such trapping mechanisms, however, do not allow for the selective targeting of individual particles and, thus, they are limited to manipulating large ensembles of molecules or colloids.

As an alternative to the localized heating of the liquid by direct light absorption, thermal gradients can be produced in the liquid adjacent to a continuous metal film that supports surface plasmon polaritons. These hybrid electromagnetic waves are characterized by enhanced optical fields that are accompanied by increased ohmic heating of the supporting metal film. Temperature gradients generated in the vicinity of plasmonic metal films have been used to initiate large-scale ordering of colloidal aggregates [454] as well as to sort gold nanoparticles in microfluidic environments [455].

In order to control the liquid motion and the associated hydrodynamic forces at the nanoscale, heating of the liquid by illuminated plasmonic nanostructures can be exploited to produce strongly localized temperature gradients that subsequently induce confined convection rolls [456-458]. Random plasmonic nano-islands have also been proposed for optical trapping and for assembling 
particles and live cells into highly organized two-dimensional patterns on planar surfaces [459] or near the tip of a gold-coated single-mode optical fiber [460]. In this context, employing highly localized temperature fields generated by plasmonic nanostructures allowed to overcome some of the limitations of optothermal trapping with focused beams and enabled the confinement and manipulation of individual nano-objects in solution (Fig. 50b) [461-463].

All previously described examples of optothermal trapping involved particles suspended in liquids. However, light-induced thermal effects can be also used to transport and confine particles in gaseous environments. This was demonstrated in [464], where individual micron-sized particles were moved and eventually trapped in air-filled hollow-core photonic crystal fibers. In these experiments, particle confinement along the fiber axis resulted from the balance of the optical radiation pressure of the guided mode and the optothermal force, which was produced by the localized heating induced by the light scattered by the particle and subsequently absorbed by the blackened fiber.

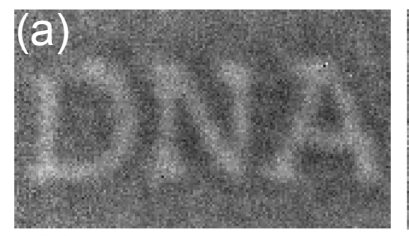

$3^{\circ} \mathrm{C}$

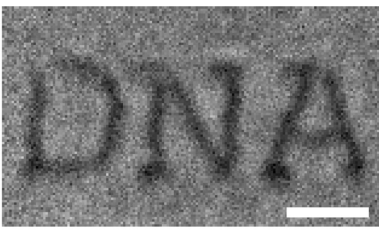

$20^{\circ} \mathrm{C}$

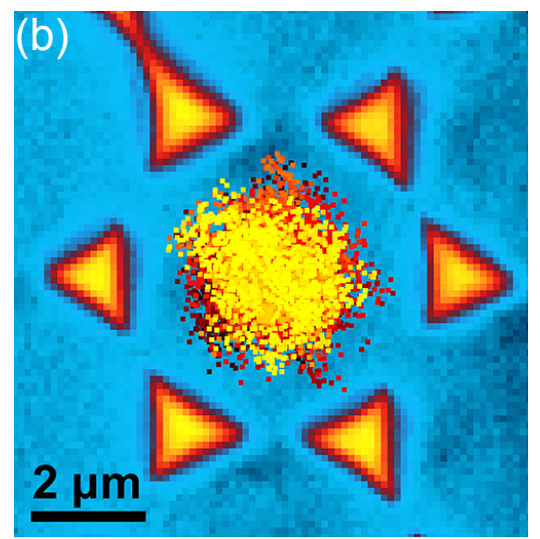

Fig. 50. Transport of particles due to light-induced thermal effects. (a) Thermodiffusion can manipulate the local concentration of DNA by small temperature differences within a bulk solution. A thin water film is heated by $2{ }^{\circ} \mathrm{C}$ along the letters "DNA" with an infrared laser. In a chamber cooled at $3{ }^{\circ} \mathrm{C}$, the fluorescently tagged DNA accumulates at the warmer letters. However, at room temperature, DNA moves into the cold regions, showing reduced fluorescence. Adapted with permission from [444]. Copyright 2006 National Academy of Sciences. (b) By creating strong local temperature gradients in liquid using optically heated gold triangular nanopads, a $200 \mathrm{~nm}$ polystyrene sphere is trapped within an open hexagonal fishnet structure at $5 \mathrm{~mW}$ heating power. The colored dots confined within the structure indicate the position of the center of mass of the sphere at different times. Adapted with permission from [461]. Copyright 2013 American Chemical Society.

\subsection{Photophoresis in absorbing particles}

In the context of optical trapping and manipulation of small particles, the thermal forces induced by light absorption within a particle (known as photophoretic forces $[23,465]$ ), and the resulting temperature gradients, have been well explored for particles suspended in a gas. In gaseous media, the photophoretic force dominates the optical manipulation of light-absorbing particles $[1,466,467]$, as it is orders of magnitude larger than the force associated with light pressure. This force originates from an uneven heating of the illuminated particles in a light field. When the particle size is much greater than the mean free path of the surrounding gas molecules, the uneven heating of the illuminated particle's surface generates a temperature gradient that induces the gas molecules to flow along the surface. The hydrodynamics of this gas motion then determines the magnitude of the photophoretic force [468]. Namely, the gas molecules on the 
warmer side of the particle leave its surface faster than the gas molecules on the colder side; this leads to a net force on the particle directed towards the colder side [23]. For strongly absorbing spherical particles, there will always be more absorption and heating at the illuminated side, thus leading to particle motion away from the light (i.e., positive photophoresis, see Fig. 51a) [23]. On the other hand, weakly absorbing spherical particles act as lenses and focus light at their non-illuminated side, which will be warmer than the opposite illuminated side; thus, they display negative photophoresis (see Fig. 51b). The dependence of the photophoretic force on the characteristics of the absorbing particles, the surrounding gas and the light field has been a subject of extensive studies [469-475]. In particular, photophoretic effects have been harnessed in light fields with complex intensity distributions in order to allow absorbing particles to be pulled into, and then trapped inside, intensity minima (Fig. 51c) [466,467,476-481] as well as to demonstrate optical tractor beams in gases [482]. For example, it was demonstrated that a speckle pattern in a spatially coherent laser field transmitted by a diffuser forms multiple three-dimensional intensity micro-pockets acting as particle traps for air-borne light-absorbing particles [483]. This photophoretic effect in speckle patterns was then adopted to perform selective trapping of light-absorbing particles in gases [477].
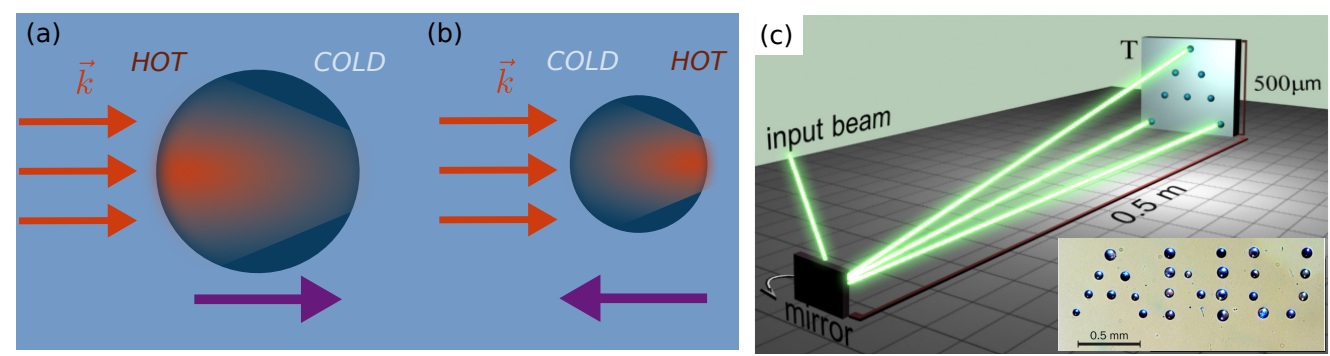

Fig. 51. Remote manipulation of absorbing particles in air due to photophoretic forces. (a) Strongly absorbing spherical particles are warmer at the illuminated side, which leads to particle motion along the light propagation direction (i.e., positive photophoresis). (b) Weakly absorbing spherical particles act as lenses, focusing the incident light at the non-illuminated side. Consequently, this side will be warmer than the opposite illuminated side due to the higher light-power density. As a result, the particles move towards the light source and display negative photophoresis. (c) Strongly absorbing particles are laterally repelled by positive photophoresis from the high-intensity ring of a vortex beam towards its low-intensity core and propelled longitudinally along the beam propagation direction. The propagation direction of the particles in a slowly diverging vortex beam is controlled by a moving mirror. The inset shows an example of the remote deposition of polydisperse glass microspheres; the letters "ANU" stands for Australian National University, and the positioning accuracy is $\pm 10 \mu \mathrm{m}$ over a distance of $0.5 \mathrm{~m}$. Adapted with permission from [478]. Copyright 2010 by the American Physical Society.

\subsection{Light-induced enhanced diffusion}

Active Brownian particles are biological or manmade microscopic and nanoscopic objects that can propel themselves by autonomously taking up energy from their environment [484]. Independently of the specific propulsion mechanism, self-propulsion generally derives from breaking the symmetry of the active particle. This leads to directed motion through either body deformations or through various self-phoretic mechanisms that maintain a stationary local gradient of, e.g., concentration, interfacial energy, or temperature [485]. In fact, at the low Reynolds numbers where the motion of the active microscopic particles take place, the absence of inertial effects requires non-reciprocal driving patterns in Newtonian liquids, such as water [486]. In the presence of a spatially homogeneous distribution of their energy source, the translational 
motion of active Brownian particles features a crossover from ballistic motion at times $t \ll \tau_{\mathrm{R}}$ to enhanced diffusion at times $t \gg \tau_{\mathrm{R}}$, with an effective diffusion coefficient that is higher than their Stokes-Einstein diffusion coefficient. The characteristic crossover timescale $\tau_{\mathrm{R}}$ is then given by the inverse of the particle's rotational diffusion coefficient [38]. These active particles have attracted a great deal of attention from the international research community since they provide innovative ways to autonomously pick-up, deliver and/or remove small cargoes, with potential applications in, e.g., personalized healthcare, environmental sustainability, and security [38]. Although various methods have been developed to achieve self-propulsion, here, we will only describe the main principles behind those methods that are initiated by light-induced local conversion of energy (Fig. 52). We refer the reader to a recent review for an extensive overview of other possible mechanisms of light-induced self-propulsion [39].
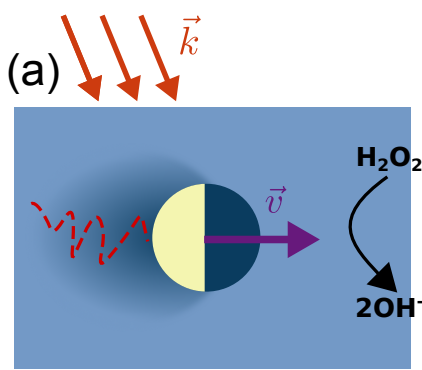
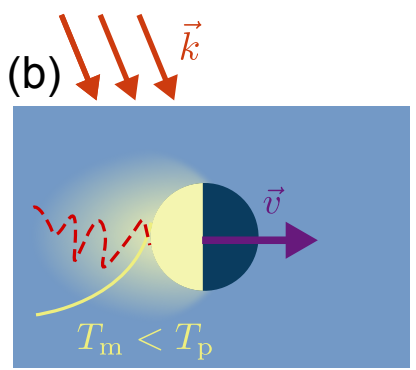
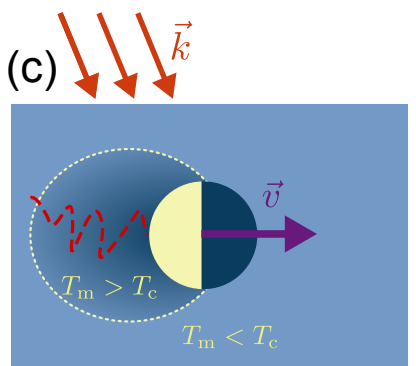
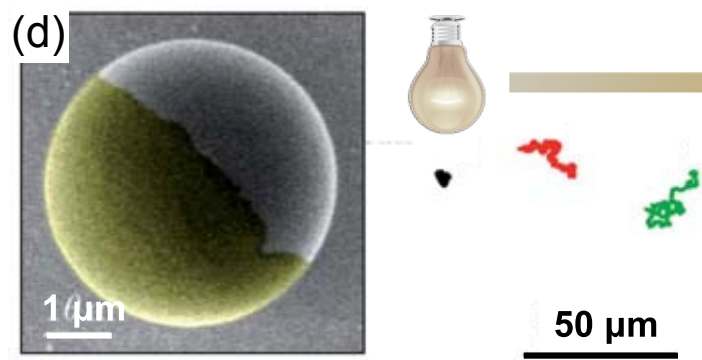

$50 \mu \mathrm{m}$

Fig. 52. Self-propulsion of light-activated Janus particles. (a-c) Main mechanisms of self-propulsion: (a) light asymmetrically powers a chemical reaction on the particle's surface, thus leading to self-diffusiophoresis; (b-c) light absorption generates a temperature gradient around the particle that makes it move (b) due to self-thermophoresis in water or (c) due to self-diffusiophoresis in a critical mixture. The direction of motion indicated by the purple arrows is only illustrative. (d) Example of light-driven self-propulsion of a Janus particle half-coated with gold, whose scanning electron microscope image is shown on the left. Light absorption at the gold cap makes the particle propel in a critical binary mixture of water and 2,6-lutidine. The representative 2-D trajectories shown on the right illustrate that the particle's motion becomes superdiffusive with an increasing power of the incident light. Adapted with permission of Royal Society of Chemistry, from [487]; permission conveyed through Copyright Clearance Center, Inc.

A very successful mechanism for the realization of microscopic self-phoretic systems is based on the so-called Janus particles (named after the two-faced Roman god). In their simplest form, these particles are prepared from dielectric colloids (made of, e.g., silica or polystyrene ) that are partially coated with a different material, most frequently a metal. The original implementation relied on thin layers of catalytic materials such as platinum or palladium to locally decompose $\mathrm{H}_{2} \mathrm{O}_{2}$ in water and create a local concentration gradient for the particle's selfdiffusiophoresis [488]. This concept has been used and modified by many other groups worldwide who also included mechanisms of light activation of a catalytic reaction [487,489-492]. Instead 
of platinum or palladium, hematite can be used as a catalyst, which has the advantage of allowing to control $\mathrm{H}_{2} \mathrm{O}_{2}$ decomposition by light; in fact, hematite catalyzes $\mathrm{H}_{2} \mathrm{O}_{2}$ decomposition only when illuminated with blue light [492]. Due to its high photocatalytic activity, titania instead of hematite was also proposed as photoactive material for similar catalytic micromotors [493-495].

When Janus particles coated with a light-absorbing material are illuminated with intense laser light, temperature gradients can also form along the particles due to the selective heating of the absorbing cap; this leads to a strong self-thermophoretic motion that can be tuned by the incident laser power, as it has been shown in the case of gold-capped colloidal particles [490,496]. The high intensity gradients associated with tightly focused illuminating beams can also lead to optical forces. Although these optical forces can interfere with the propulsion mechanism [497], they can also be used to remotely maneuver the particles $[498,499]$. Differently from self-thermophoresis, which requires sufficiently high light intensities, much lower intensities are needed for actuating Janus particles immersed in binary liquid mixtures with a lower critical point. When the mean temperature of the mixture is kept sufficiently close to its critical value, even for small illumination intensities, light absorption at the absorbing cap leads to local heating, which results in a local phase separation and, consequently, in diffusiophoresis induced by a concentration gradient across the particle [487,500]. Due to the much smaller light intensities compared to the thermophoretic mechanisms described above, optical forces are typically negligible in this actuation scheme. The microscopic details behind this propulsion mechanism are still debated and different possibilities have been recently put forward theoretically [501,502].

Moreover, similarly to biological entities, such as phototactic bacteria and algae [503-506], artificial Janus particles can also show directional phototactic behavior when moving in a light intensity gradient rather than in a homogeneous light field [507,508].

Finally, complex transport behaviors can also emerge when individual units (either capable of self-propulsion or not) self-assemble to form larger clusters in response to light-induced thermophoretic motion [492,509-511].

\subsection{Propulsion by light-induced body deformation}

The asymmetry needed for self-propulsion can also be achieved by a periodic, non-reciprocal geometrical deformation or reorientation of the particle's body. At the micrometer scale, this has, for example, been shown by applying a time-dependent external field that induces forces and mechanical torques on the object. In the context of optical manipulation, a system of three simultaneously trapped colloidal particles was forced to move through a cycle that breaks time-reversal symmetry by varying inter-particle distance according to a specified pattern [512].

Alternatively, deformation of light-responsive polymers is an interesting strategy to achieve motion. Although other materials are possible [513,514], a particularly well-explored strategy relies on the use of hybrid materials known as liquid crystalline elastomers (LCE) [515-517]. This strategy has already proven successful at the millimeter scale [517-520]. Structures made of these materials have been downscaled to the sub-millimeter range and have served as miniature walkers on air-solid interface [521] or as swimming soft micro-robots [522] (see Fig. 53).

\subsection{Transport by optically induced capillary effects and Marangoni stresses}

Liquid microdroplets represent a special class of "soft" particles that form due to interfacial tension between immiscible liquids. They can serve as miniature transport containers and reaction vessels for applications in chemistry, biology, and medicine [523]. The recent boom in digital microfluidics - the research field dealing with the generation, manipulation, mixing, and compositional analysis of microdroplets [524] - has stimulated a steadily growing interest in the selective manipulation of individual droplets.

The immiscible liquids used for droplet generation (typically a combination of water and various types of mineral or organic oils) often display a contrast of dielectric properties/refractive 

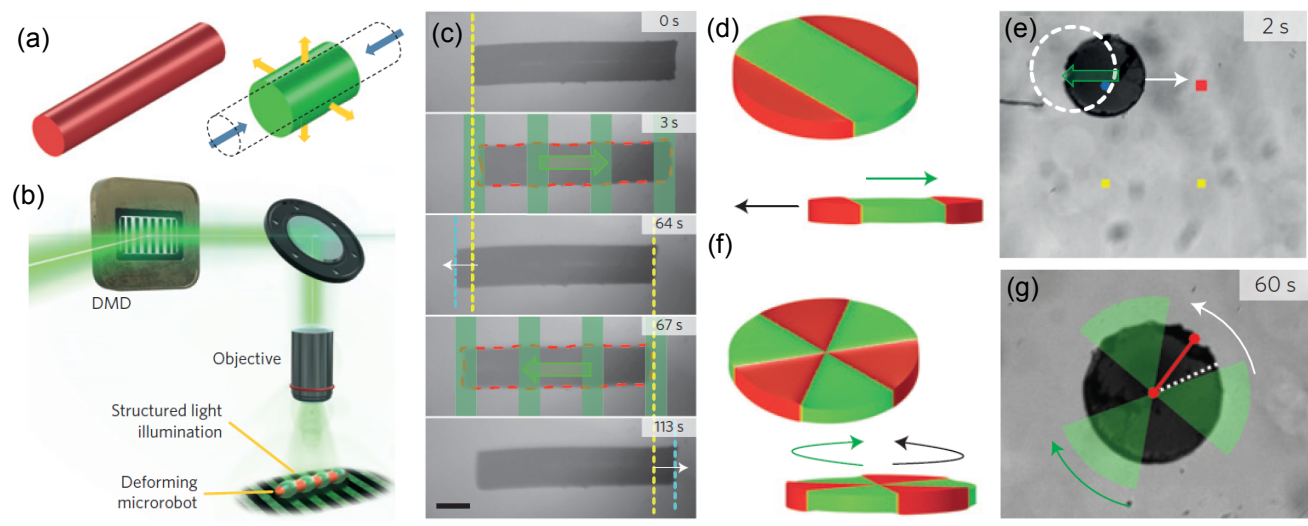

(f)
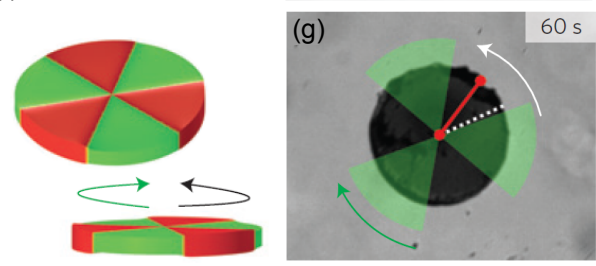

Fig. 53. Swimming micro-robots. (a) Illustration of a cylindrical liquid crystal elastomer at rest (left) and after deformation initiated by light at $532 \mathrm{~nm}$ (right). (b) The micro-robot is illuminated with a linear interference pattern generated with a digital micro-mirror device (DMD). Only the illuminated part of the micro-robot is deformed. (c) When the intensity pattern moves to the right (top) or left (bottom), the micro-robot moves in the opposite direction. The yellow and cyan dashed lines denote the initial position and the final position, respectively. (d-e) When a disk-shaped micro-robot is illuminated with a linear interference pattern, it moves in the opposite direction of the light pattern shift. (f-g) When the disk-shaped micro-robot is illuminated with an azimuthal rotating light pattern, it starts to rotate instead in the opposite direction. Adapted by permission from Springer Nature Customer Service Centre GmbH: Nature Materials [522], Copyright 2016.

index that renders them amenable for manipulation by the dielectrophoretic forces [434] and optical gradient forces $[525,526]$ discussed above. Alternatively, droplets deposited on a solid surface or suspended in a host liquid can be actuated by forces arising from gradients of surface wettability or of surface tension (Marangoni effect) [527,528]. In particular, such gradients of surface energy can be produced optically, e.g., by illuminating the droplet surface and harnessing the thermal or photochemical effects of the light absorbed within the droplet [529,530]. Similarly, on the microscale, localized heating produced by a focused laser beam can indirectly route droplets in microfluidic devices by giving rise to thermocapillary forces due to Marangoni stresses [531-533]

Figure 54 illustrates the origins of the optically induced capillary forces. Asymmetric illumination of a sessile droplet deposited on a substrate with photosensitive wettability leads to a difference of contact angles between the illuminated $\left(\theta^{+}\right)$side and the dark $\left(\theta^{-}\right)$side of the droplet (see Fig. 54a). The difference in surface curvature between these two sides then produces a gradient of Laplace pressure that pushes the droplet in the direction of the lower of the two contact angles $\theta^{+}, \theta^{-}$. Intuitively, the droplet moves towards the side with the lower contact angle because the surface is more wettable there. Practically, surfaces with photosensitive wettability typically involve thin organic films deposited on solid substrates. For this purpose, the most widely used photochromic coatings are based on azobenzene molecules and their derivatives. These molecules can be reversibly switched between two molecular conformations with different polarity upon illumination with UV $(\sim 360 \mathrm{~nm})$ and blue $(\sim 450 \mathrm{~nm})$ light [530]. The pioneering experiments presented in [534] achieved fully reversible millimeter-range motion of oil droplets on a substrate coated with azobenzene-modified calixarene under illumination with an intensity gradient of blue light. The droplets could be moved along a straight line in both directions, with typical speeds of $\sim 35 \mu \mathrm{m} . \mathrm{s}^{-1}$ and no appreciable deterioration in the actuation performance even after 100 cycles (see Fig. 55a). Similar linear droplet's motion was also later reported in [535] 

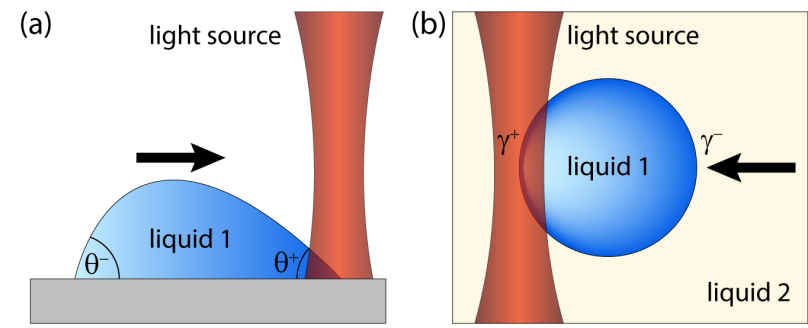

Fig. 54. Optically induced actuation of droplets. (a) The contact angle of a sessile droplet on a solid surface is modified by illumination with light of a suitable wavelength. Due to the asymmetric illumination, differences in contact angles $\theta^{+}, \theta^{-}$at the opposite sides of the droplet lead to a gradient of Laplace pressure between the locations of low $\left(\theta^{+}\right)$and high $\left(\theta^{-}\right)$ surface curvature. The resulting pressure-driven flow then pushes the droplet in the direction of the arrow. (b) Due to the asymmetric illumination, differences in surface tensions $\gamma^{+}, \gamma^{-}$ at the opposite sides of the droplet lead to a Marangoni flow directed from the low- to the high-surface-tension region. If $\gamma^{+}<\gamma^{-}$, viscous Marangoni flows drag the surrounding liquid (liquid 2) away from the illuminated side. Because of momentum conservation for the two liquids (liquids 1 and 2), the droplet then moves towards the illuminated side.

and [536].

(a)

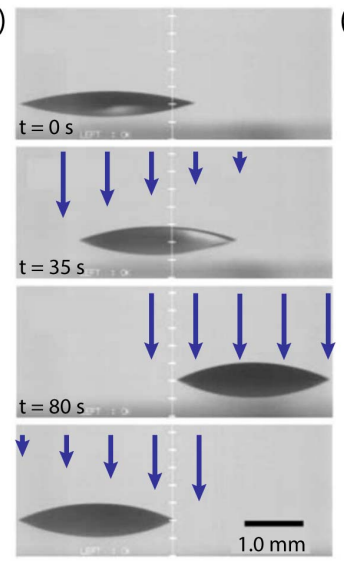

(b)

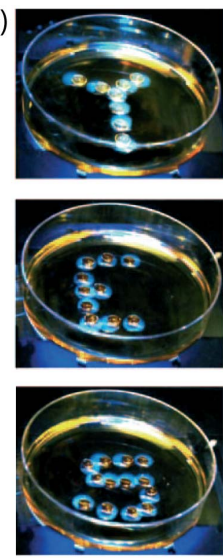

Fig. 55. Examples of droplet manipulation via optically induced capillary forces.

(a) Light-driven motion of an olive oil droplet on a silica plate coated with azobenzenemodified calixarene under illumination with an intensity gradient of blue light (arrows). The droplet's direction of motion can be reversed by changing the orientation of the intensity gradient. Adapted with permission from [534]. Reprinted with permission from AAAS. (b) Photomontages of the motion of a droplet along complex trajectories reproducing the letters "Y", "E", and "S". The droplet is held in a chromocapillary trap during the motion. Adapted with permission from [537]. Copyright 2009 John Wiley and Sons.

Droplets and bubbles fully suspended in a host liquid can also be confined and manipulated by adjusting their surface tension. As shown in Fig. 54b, the selective illumination of the droplet surface can lead to a local modification of surface tension $\gamma$. The resulting gradient in $\gamma$ then initiates an interfacial fluid flow directed along this gradient. This phenomenon, known as the Marangoni effect, has been exploited for fluid actuation in a number of microfluidic applications [538]. In the example shown in Fig. 54b, the value of $\gamma^{+}$at the illuminated side is lower than the value of $\gamma^{-}$at the dark side so that the interfacial flow is directed towards the 
dark side. Due to viscous stresses, the liquid surrounding the droplet is dragged along with this interfacial flow. Since the total momentum of the system has to be conserved, the droplet will move in the opposite direction of this flow and towards the illumination beam. Intuitively, since the value of $\gamma$ is lower at the illuminated side, the system tends to minimize its overall energy by moving towards the illumination beam.

An early demonstration of light-induced thermocapillary manipulation of individual emulsion droplets was presented in 2004 [539]. In these experiments, a green laser beam was focused at one side of an aqueous droplet suspended in decanol. Under illumination, the droplets were pushed away from the laser beam, thus indicating that $\gamma^{+}>\gamma^{-}$for that particular combination of liquids. The droplets could be moved through the surrounding liquid at a speed up to $\sim 3 \mathrm{~mm} . \mathrm{s}^{-1}$. In [540], a complete "optical toolbox" was proposed and implemented that used a focused laser beam for controlling the formation, transport, division and fusion of aqueous droplets flowing in a microfluidic chip in a mixture of hexadecane and Span 80 surfactant. All these microfluidic operations were mediated by thermocapillary forces in an emulsion where the value of $\gamma$ increased with temperature, i.e., $\gamma^{+}>\gamma^{-}$. The origin of this anomalous behavior (typically, $\gamma$ decreases with rising temperature) was later explained by the complex transport patterns of the surfactant micelles in the vicinity of the illuminated spot on the droplet surface [541]. Using the same emulsion system and experimental procedure, fast droplet switchers and sorters for applications in digital microfluidics were demonstrated [542]. By employing an absorbing substrate made with layers of ITO and amorphous silicon, the simultaneous manipulation of multiple droplets was achieved by inducing thermocapillary forces. These forces were obtained by illuminating the substrate with a light pattern generated with a conventional computer projector [543].

In addition to manipulating liquid droplets, thermocapillary forces have been also harnessed to actuate solid rotary micromotors [533]. In these experiments, a Marangoni flow was employed to apply a mechanical torque to an asymmetric micron-sized rotor capable of spinning at rates up to 300 r.p.m. under wide-field illumination with incoherent light, with the rate of rotation quickly and indefinitely controllable by tuning the power of the incident light.

As an alternative to exploiting thermal effects associated with light absorption, optical control of surface tension can also be achieved through the use of photosensitive surfactants. These surface-active compounds change polarity or redistribute in space upon illumination with light of a suitable wavelength (typically in the UV or in the blue spectral region), thus creating spatial gradients of surface tension. In principle, it is possible to control interfacial energy isothermally, which is beneficial for temperature-sensitive applications such as those involving live cells or delicate biomolecules. In [537], a scheme was devised for the omnidirectional manipulation of oil droplets floating on the surface of an aqueous solution of AzoTAB surfactants. This small molecule could be reversibly switched between two conformations of different polarity by subsequent illumination with UV (365 nm) and blue $(475 \mathrm{~nm})$ light. By changing the wavelength of the light used to illuminate the droplet's edge, it was therefore possible to reversibly repel the droplet from the incident beam (UV illumination) or attract the droplet towards it (blue illumination). Using concentric two-color illumination patterns, a "chromocapillary trap" was created and used to manipulate the floating droplets along complex trajectories (see Fig. 55b). A different strategy was adopted in [544] for achieving fast, long-distance transport of millimetersized dichloromethane (DCM) droplets. These droplets contained a pH-sensitive surfactant complex and rested on the surface of an aqueous solution of spiropyran sulfonic acid confined within an open fluidic channel. Upon inhomogeneous illumination of the channel with white light from an LED, a gradient of $\mathrm{pH}$ in the photosensitive acid solution could be reversibly generated. Since the opposite sides of the DCM droplets were exposed to different local values of $\mathrm{pH}$, the surfactant was released from the droplet at different rates at these locations and, subsequently, migrated towards and accumulated at the water-air interface. The difference in the local surfactant concentration immediately outside the droplet then led to a corresponding 
difference in air-water surface tension at opposite sides of the droplet. Consequently, the droplet moved in the direction of increasing surface tension, in an analogy to the classic camphor boat experiment [545]. Depending on the intensity and spatial profile of the illuminating light, the DCM droplets could be moved over several centimeters with speeds up to $4 \mathrm{~mm} \cdot \mathrm{s}^{-1}$.

With the particular liquid/surfactant system used in [544], the droplets moved away from the light source (negative phototaxis). Similar light-induced repulsive forces were produced in an oil-in-water emulsion system based on $n$-heptyloxybenzaldehyde and stabilized by a photoresponsive cationic surfactant containing an azobenzene moiety [546]. However, a different choice of the liquids for the droplet and its surrounding medium as well as of the photosensitive surfactant can lead to positive phototaxis, i.e., the droplet seeking the location of the highest optical intensity. For example, this behavior was observed in experiments reported in [547], in which emulsion droplets of 2-nitrobenzyl oleate (NBO) suspended in water and illuminated asymmetrically with $365 \mathrm{~nm}$ light moved towards the light source. Here, the necessary gradient of surface tension was generated by local photo-dissociation of NBO yielding negatively charged oleate acting as a surfactant.

\section{Conclusions and outlook}

In this review, we have covered the majority of light-induced physical mechanisms and experimental arrangements that can be exploited for initiating controlled, directional transport of illuminated microobjects. Delicate optical forces that act upon materials exposed to light have been a subject of intense theoretical and experimental research from interdisciplinary scientific communities for almost fifty years. The main focus has been on the use of these forces for the controlled confinement, manipulation, and transport of small particles, molecules, and atoms. Over this period of time, the field has significantly evolved and expanded. The initial proof-of-principle experiments of Arthur Ashkin, which demonstrated the forces induced by light, have led to applications in research disciplines as diverse as physics, materials science, chemistry and medicine. Using the direct transfer of linear momentum from photons, it is currently possible to move an illuminated microobject along arbitrary 3-D trajectories, including those pointing against the direction of propagation of the incident light. The state of motion of an object placed into an optical field possessing angular momentum can be even more complex, especially for objects with some degree of material or shape anisotropy. By exploiting the reduced dimensionality of the space accessible to an illuminated object, physical interfaces allow for microobjects to be sorted efficiently. Optical forces can be complemented by other types of forces of non-optical origin that scale differently with the material properties, size, or shape of the objects, thus increasing the number of available tools for the selective manipulation and transport of objects. Furthermore, the energy carried by light can be converted by the medium surrounding the target object or by the object itself to produce gradients of electric potential, $\mathrm{pH}$, concentration or temperature, which can subsequently lead to more efficient transport per watt of optical power incident on the object.

Among the various optical micromanipulation schemes discussed in the article, we would like to highlight several particular directions, which - in our opinion - open doors to new realms and exciting future applications of light-induced transport:

\section{- Optical fibers}

Optical fibers can be exploited for transporting particles and atoms over macroscopic distances (tens of centimeters), along trajectories that are spatially confined either to the proximity of the fiber surface [379] or to the channels forming the internal structure of hollow-core photonic crystal fibers [398,399, 548]. New types of hollow-core [549], multicore, or microstructured $[550,551]$ fibers with unique light-guiding properties suitable for particle confinement and actuation are continuously being developed. Furthermore, the fiber surface can be micromachined [552] and fibers with complex architectures can 
be manufactured in a fast and cheap way using a 3-D printer [553]. Fiber-based particle transport can be integrated with other functionalities of the fibers, e.g., optical imaging or spectroscopy [554], leading to new types of fiber-based endoscopes. These tools could be used to inspect a well-localized area of a tissue with minimal intervention, and at the same time to deliver biologically functionalized objects, cells or encapsulated drugs in a sterile way [305]. However, the necessity of direct mechanical access of the fiber to the area of delivery can be viewed as a potential limitation for fiber-based transport in certain applications.

\section{- Optofluidic chips}

Optofluidic chips combine advantages from two worlds for the implementation of integrated lab-on-a-chip devices and systems: besides allowing for the flexible, contactless, sterile light-induced transport of solid particles, living bacterial and eukaryotic cells, microdroplets and biomolecules suspended in a suitable host liquid, they also exploit recent technological advances in the micro- and nanofabrication of complex fluidic channel architectures for the precise shaping of the light waves propagating within the chips. Such optofluidic chips can be employed to perform specific tasks, e.g., the characterization of the mechanical properties of individual biomolecules [376], the control of chemical reactions taking place in emulsion microdroplets flowing through the chip [555], the determination of the material characteristics of the liquid within a droplet [556], or the selective detection of nanoparticles or viruses [557]. The implementation of plasmonic surfaces [12] and metasurfaces [558] within the chips can enhance their performance for particle trapping and transport and their sensitivity in discriminating between different types of particles.

\section{- Optically initiated nanoprinting}

Optical nanoprinting provides a versatile and cheap platform for the surface deposition of metal or dielectric particles into arbitrary configurations with nanometric precision. This technique employs different physical mechanisms, some of which (e.g., optical printing based on optical forces, optically induced dielectrophoresis, and photophoresis) have been already mentioned in our review. A more extensive list can be found elsewhere [442,559]. Up to now, optical nanoprinting has achieved the deposition of individual plasmonic nanoparticles [135, 139, 143, 146, 560] and nanoparticles in dimer configurations [561], linear and V-shaped metal nanorods $[148,153,154,562]$ as well as other metallic particles of complex shape [152], silicon nanoparticles [155,563], multiple particles in parallel [161], and Janus particles [564]. Optical nanoprinting could, therefore, accelerate the recent transition from plasmonic nanoantennas and metamaterials to high-refractiveindex all-dielectric nanoantennas and metasurfaces that offer lower dissipative losses and, consequently, lower heating. Such dielectric metamaterials and metasurfaces are expected to enable the implementation of, e.g., high-refractive index nanoresonators or diffractiion gratings, as well as devices for the directed and polarization-sensitive transmission of light and for the generation of higher-harmonics waves in nonlinear optics [156-158,565].

\section{- Space propulsion}

Light sails have been envisioned for decades as an ideal way for satellite propulsion across the galaxies [123] and, as we have already mentioned in the introduction of Section 3, several practical tests have been already performed. Transport in free space, with negligible gas pressure, represents a mode of operation different from the overdamped motion in liquids or air, as the inertia of the object has to be taken into account. The radiation from the Sun provides a perpetual source of propulsive force; unfortunately, it is very weak and has to be compensated by large sail dimensions. In contrast, the Breakthrough 
Starshot Initiative plans to propel a nanosatellite towards Proxima Centauri using the strong radiation pressure generated by a phased laser array, which would be placed on Earth and act only over a limited period of time [129]. Thus, this operational mode would fully exploit the satellite inertia. However, in order to succeed, complex engineering challenges must be first solved. These include, e.g., the balancing of the competing demands on lightweightness and robustness of the sail, the angular stabilization of the satellite during acceleration, the high reflectivity and low absorption of the sail material over the spectral interval that covers the wavelength of the accelerating laser beams while it is gradually Doppler-shifted due to the satellite's motion at speeds of up to $20 \%$ of the speed of light, and the high emissivity of the sail at thermal wavelengths to radiate the absorbed energy of the propulsion laser beams $[130,131,566]$.

\section{- Vacuum levitation}

Recent progress in optomechanics [567], especially in the levitation of objects optically confined in vacuum [568], promises a fundamental quantitative increase in the detection sensitivity of ultraweak forces [569-571] and torques [572-574], opening of new paths to experimental realization of microscopic stochastic systems that are far from equilibrium and whose phase space can be fully externally controlled [575,576], as well as a qualitative technological push towards quantum technologies based on optically cooled center-of-mass motion of levitated nanoobjects [258,303,571,577-582]. Downsizing the experimental platforms from conventional bulky vacuum chambers to vacuum chips represents a critical step towards their practical utilization $[304,583]$.

\section{- Light as a trigger}

Beyond the topics covered in Section 5, additional strategies for light-induced particle transport can be envisaged using light to trigger direct or indirect energy conversion in the particles or their surrounding environment. Particularly alluring is the possibility of developing innovative, controllable, non-equilibrium mechanisms where light triggers the self-propulsion of particles capable of spontaneous local energy conversion [39]. Light is in fact an energy source with desirable properties to drive micro- and nanorobots as it is readily controllable, broadly biocompatible and it can be made widely available thanks to recent advances in wavefront shaping. For example, the possibility of sensing the spatial distribution of the optical intensity in complex light fields could allow individual self-propelled particles to navigate their environment efficiently, thus mimicking the phototactic response of many microorganisms [507]. Similar sensing capabilities could be exploited to achieve the reversible self-assembly of units as a tool to study the collective dynamics of non-equilibrium systems and for synthesizing new materials and metamaterials with tailored internal structure and properties [584,585]. Additionally, combining such light-triggered phoretic motion with force fields of other origin (e.g., acoustic, magnetic and electric fields) has the potential to reduce the drawbacks associated with the use of each individual mechanism, thus enabling hybrid transport functionalities and sensing capabilities.

\section{Acknowledgement}

P.Z., O.B. and A.J. acknowledge financial support from the Czech Academy of Sciences, Czech Science Foundation (project 19-17765S) and Ministry of Education, Youth and Sports of the Czech Republic (LO1212). 


\section{References}

1. A. Ashkin, "Acceleration and trapping of particles by radiation pressure," Phys. Rev. Lett. 24, 156-159 (1970).

2. A. Ashkin and J. M. Dziedzic, "Optical levitation by radiation pressure," Appl. Phys. Lett. 19, 283-285 (1971).

3. A. Ashkin and J. M. Dziedzic, "Stability of optical levitation by radiation pressure," Appl. Phys. Lett. 24, 586-588 (1974).

4. https://www.nobelprize.org/prizes/physics/2018/summary/.

5. https://www.nobelprize.org/prizes/physics/1997/summary/.

6. https://www.nobelprize.org/prizes/physics/2001/summary/.

7. A. Ashkin, J. M. Dziedzic, J. E. Bjorkholm, and S. Chu, "Observation of a single-beam gradient force optical trap for dielectric particles," Opt. Lett. 11, 288-290 (1986).

8. S. Kawata and T. Sugiura, "Movement of micrometer-sized particles in the evanescent field of a laser beam," Opt. Lett. 17, 772-774 (1992).

9. D. Erickson, X. Serey, Y.-F. Chen, and S. Mandal, "Nanomanipulation using near field photonics," Lab Chip 11, 995-1009 (2011).

10. K. Dholakia and W. M. Lee, "Optical trapping takes shape: The use of structured light fields," Adv. At. Mol. Opt. Phys. 56, 261-337 (2008).

11. M. Woerdemann, C. Alpmann, M. Esseling, and C. Denz, "Advanced optical trapping by complex beam shaping," Laser Photon. Rev. 7, 839-854 (2013).

12. M. L. Juan, M. Righini, and R. Quidant, "Plasmon nano-optical tweezers," Nat. Photonics 5, 349-356 (2011).

13. O. Brzobohatý, V. Karásek, M. Šiler, L. Chvátal, T. Čizmár, and P. Zemánek, "Experimental demonstration of optical transport, sorting and self-arrangement using a 'tractor beam'," Nat. Photonics 7, 123-127 (2013).

14. D. B. Ruffner and D. G. Grier, "Optical conveyors: A class of active tractor beams," Phys. Rev. Lett. 109, 163903 (2012).

15. A. Terray, J. Taylor, and S. Hart, "Cascade optical chromatography for sample fractionation," Biomicrofluidics 3 , 044106:1-6 (2009).

16. M. P. MacDonald, G. C. Spalding, and K. Dholakia, "Microfluidic sorting in an optical lattice," Nature 426, 421-424 (2003).

17. G. Thalhammer, R. Steiger, M. Meinschad, M. Hill, S. Bernet, and M. Ritsch-Marte, "Combined acoustic and optical trapping," Biomed. Opt. Express 2, 2859-2870 (2011).

18. G. Pesce, G. Rusciano, G. Zito, and A. Sasso, "Simultaneous measurements of electrophoretic and dielectrophoretic forces using optical tweezers," Opt. Express 23, 9363-9368 (2015).

19. E. Martínez, I.A. ad Roldán, J. Parrondo, and D. Petrov, "Effective heating to several thousand kelvins of an optically trapped sphere in a liquid," Phys. Rev. E 87, 032159 (2013).

20. H. Park and T. W. LeBrun, "Parametric force analysis for measurement of arbitrary optical forces on particles trapped in air or vacuum," ACS Photonics 2, 1451-1459 (2015).

21. A. Jonáš and P. Zemánek, "Light at work: The use of optical forces for particle manipulation, sorting, and analysis." Electophoresis 29, 4813-4851 (2008).

22. M. C. Wu, "Optoelectronic tweezers," Nat. Photonics 5, 322-324 (2011).

23. O. Jovanovic, "Photophoresis - light induced motion of particles suspended in gas," J. Quant. Spectrosc. Ra. 110, 889 - 901 (2009).

24. D. G. Grier, “Optical tweezers in colloid and interface science,” Curr. Opin. Colloid. Interf. 2, 264-270 (1997).

25. I. A. Martinez, E. Roldan, L. Dinis, and R. A. Rica, "Colloidal heat engines: a review," Soft Matter 13, 22-36 (2017).

26. D. Kumar, S. Bhattacharya, and S. Ghosh, "Weak adhesion at the mesoscale: particles at an interface," Soft Matter $\mathbf{9}$, 6618-6633 (2013).

27. S. Mohanty, "Optically-actuated translational and rotational motion at the microscale for microfluidic manipulation and characterization,” Lab Chip 12, 3624-3636 (2012).

28. H. C. Hunt and J. S. Wilkinson, "Optofluidic integration for microanalysis," Microfluid. Nanofluid. 4, 53-79 (2008).

29. R. Power and J. Reid, "Probing the micro-rheological properties of aerosol particles using optical tweezers," Rep. Prog. Phys. 77, 074601 (2014).

30. D. V. Petrov, "Raman spectroscopy of optically trapped particles," J. Opt. A: Pure Appl. Opt. 9, S139-S156 (2007).

31. J. P. Reid, H. Meresman, L. Mitchem, and R. Symes, "Spectroscopic studies of the size and composition of single aerosol droplets," Int. Rev. Phys. Chem. 26, 139-192 (2007).

32. N. Kitamura and F. Kitagawa, "Optical trapping - chemical analysis of single microparticles in solution," J. Photochem. Photobiol. C 4, 227-247 (2003).

33. S. Juodkazis, V. Mizeikis, S. Matsuo, K. Ueno, and H. Misawa, "Three-dimensional micro- and nano-structuring of materials by tightly focused laser radiation," Bull. Chem. Soc. Japan 81, 411-448 (2008).

34. D. Palima and J. Glückstad, "Generalized phase contrast matched to Gaussian illumination,” Laser Photon. Rev. 7, 478-494 (2013).

35. K. Ramser and D. Hanstorp, "Optical manipulation for single-cell studies," J. Biophot. 3, 187-206 (2010).

36. N.-T. Huang, H.-1. Zhang, M.-T. Chung, J. H. Seo, and K. Kurabayashi, "Recent advancements in optofluidicsbased single-cell analysis: optical on-chip cellular manipulation, treatment, and property detection," Lab Chip 14, 1230-1245 (2014).

37. E. M. Darling and D. Di Carlo, "High-Throughput Assessment of Cellular Mechanical Properties," in "Annual 
Review of Biomedical Engineering, Vol 17,", vol. 17 of Annual Review of Biomedical Engineering, Yarmush, ML, ed. (2015), pp. 35-62.

38. C. Bechinger, R. Di Leonardo, H. Löwen, C. Reichhardt, G. Volpe, and G. Volpe, "Active particles in complex and crowded environments," Rev. Mod. Phys. 88, 045006 (2016).

39. L. Xu, F. Mou, H. Gong, M. Luo, and J. Guan, "Light-driven micro/nanomotors: from fundamentals to applications," Chem. Soc. Rev. 46, 6905-6926 (2017).

40. A. Ashkin, "Applications of laser radiation pressure," Science 210, 1081-1088 (1980).

41. K. Svoboda and S. M. Block, "Biological applications of optical tweezers," Ann. Rev. Biophys. Biomol. Struct. 23, 247-285 (1994).

42. A. Ashkin, "Optical trapping and manipulation of neutral particles using lasers," Proc. Natl. Acad. Sci. USA 94, 4853-4860 (1997).

43. M. J. Lang and S. M. Block, "Resource letter: LBOT-1: Laser-based optical tweezers," Am. J. Phys. 71, 201-215 (2003).

44. K. C. Neuman and S. M. Block, “Optical trapping,” Rev. Sci. Instr. 75, 2787-2809 (2004).

45. S. M. Block, "Optical tweezers: A new tool for biophysics," in "Noninvas. Tech. in Biol. (Mod. Rev. Cell Biol. 9),", J. Foskett and S. Grinstein, eds. (Wiley, New York, 1990), pp. 375-402.

46. D. G. Grier, “A revolution in optical manipulation,” Nature 424, 810-816 (2003).

47. K. Dholakia, P. Reece, and M. Gu, "Optical micromanipulation," Chem. Soc. Rev. 35, 42-55 (2008).

48. X. Miao and L. Y. Lin, “Trapping and manipulation of biological particles through a plasmonic platform," IEEE J. Sel. Top. Quant. Electr. 13, 1655-1662 (2007).

49. R. Quidant and C. Girard, "Surface-plasmon-based optical manipulation," Laser Photon. Rev. 2, 47-57 (2008).

50. R. W. Bowman and M. J. Padgett, “Optical trapping and binding," Rep. Prog. Phys. 76, 026401 (2013).

51. M. Padgett and R. Bowman, "Tweezers with a twist," Nat. Photonics 5, 343-348 (2011).

52. K. Dholakia and T. Čižmár, "Shaping the future of manipulation," Nat. Photonics 5, 335-342 (2011).

53. F. M. Fazal and S. M. Block, "Optical tweezers study life inder tension," Nat. Photonics 5, 318-321 (2011).

54. A. D. Mehta, M. Rief, J. A. Spudich, D. A. Smith, and R. M. Simmons, "Single-molecule biomechanics with optical methods," Science 283, 1689-1695 (1999).

55. S. H. Simpson, "Inhomogeneous and anisotropic particles in optical traps: Physical behaviour and applications," J. Quant. Spectrosc. Radiat. Transf. 146, 81-99 (2014).

56. K. C. Neuman and A. Nagy, "Single-molecule force spectroscopy: optical tweezers, magnetic tweezers and atomic force microscopy," Nat. Methods 5, 491-505 (2008).

57. J. R. Moffitt, Y. R. Chemla, S. B. Smith, and C. Bustamante, "Recent advances in optical tweezers," Ann. Rev. Biochem. 77, 205-228 (2008).

58. W. J. Greenleaf, M. T. Woodside, and S. M. Block, "High-resolution, single-molecule measurements of biomolecular motion," Annu. Rev. Biophys. Biomol. Struct. 36, 171-190 (2007).

59. C. Bustamante, J. Macosko, and G. Wuite, "Grabbing the cat by the tail: Manipulating molecules one by one," Nature Reviews Molecular Cell Biology 1, 130-136 (2000).

60. J. E. Molloy and M. J. Padgett, "Light, action: optical tweezers," Contemp. Phys. 43, 241-258 (2002).

61. M. Dienerowitz, M. Mazilu, and K. Dholakia, "Optical manipulation of nanoparticles: A review," J. Nanophotonics 2, 021875:1-32 (2008).

62. L. Mitchem and J. P. Reid, "Optical manipulation and characterisation of aerosol particles using a single-beam gradient force optical trap,” Chem. Soc. Rev. 37, 756-769 (2008).

63. M. Padgett and R. Di Leonardo, "Holographic optical tweezers and their relevance to lab on chip devices," Lab Chip 11, 1196-1205 (2011).

64. T. Perkins, "Optical traps for single molecule biophysics: a primer," Laser Photon. Rev. 3, 203-220 (2009).

65. A. Yao, M. Tassieri, M. Padgett, and J. Cooper, "Microrheology with optical tweezers," Lab Chip 9, 2568-2575 (2009).

66. C. Monat, P. Domachuk, C. Grillet, M. Collins, B. Eggleton, M. Cronin-Golomb, S. Mutzenich, T. Mahmud, G. Rosengarden, and A. Mitchell, "Optofluidics: A novel generation of reconfigurable and adaptive compact architectures," Microfluid. Nanofluid. 4, 81-95 (2008).

67. T. A. Nieminen, G. Knoner, N. R. Heckenberg, and H. Rubinsztein-Dunlop, "Physics of optical tweezers," Methods in cell biology 82, 207-236 (2007).

68. L. Oddershede, "Force probing of individual molecules inside the living cell is now a reality," Nature Chem. Biol. 8, 879-886 (2012).

69. D. Ou-Yang and M.-T. Wei, "Complex fluids: Probing mechanical properties of biological systems with optical tweezers," Annu. Rev. Phys. Chem. 61, 421-440 (2010).

70. C. Pacoret and S. Regnier, "Invited Article: A review of haptic optical tweezers for an interactive microworld exploration,” Rev. Sci. Instrum. 84, 081301 (2013).

71. C.-W. Qiu, D. Palima, A. Novitsky, D. Gao, W. Ding, S. V. Zhukovsky, and J. Gluckstad, "Engineering light-matter interaction for emerging optical manipulation applications," Nanophotonics 3, 181-201 (2014).

72. S. Sukhov and A. Dogariu, "Non-conservative optical forces," Rep. Prog. Phys. 80, 112001 (2017).

73. A. Ashkin, Optical trapping and manipulation of neutral particles using lasers (World Scientific, London, 2006).

74. D. Andrews, ed., Structured Light and its Applications (Elsevier Acad. Press., 2008). 
75. M. P. Sheetz, L. Wilson, and P. Matsudaira, eds., Laser tweezers in cell biology, vol. 55 of Methods in cell biology (Academic Press, San Diego, 1998).

76. K.-O. Greulich, Micromanipulation by light in biology and medicine (Birkhauser Verlag, Basel-Boston-Berlin, 1999).

77. M. W. Berns and K. O. Greulich, eds., Laser manipulation of cells and tissues, vol. 82 of Methods in cell biology (Academic Press, San Diego, 2007).

78. G. Gouesbet and G. Gréhan, Generalized Lorenz-Mie Theories (Springer, 2011).

79. T. Wriedt and W. Hergert, The Mie Theory: Basics and Applications (Springer-Verlag, New York, 2012).

80. M. Padgett, J. Molloy, and D. McGloin, Optical tweezers: Methods and Applications (CRC Press, New York, 2010).

81. P. Jones, O. Maragò, and G. Volpe, Optical tweezers: Principles and Applications (Cambridge University Press, Cambridge, 2015).

82. M. Tassieri, Microrheology with Optical Tweezers: Principles and Applications, Microrheology with Optical Tweezers: Principles and Applications (Pan Stanford Pub, 2016).

83. A. Gennerich, Optical Tweezers: Methods and Protocols, Methods in Molecular Biology (Springer New York, 2017).

84. J. Guck, R. Ananthakrishnan, T. J. Moon, C. C. Cunningham, and J. Käs, "Optical deformability of soft biological dielectrics," Phys. Rev. Lett. 84, 5451-5154 (2000).

85. J. Guck, R. Ananthakrishnan, H. Mahmood, T. Moon, C. Cunningham, and J. Kas, "The optical stretcher: A novel laser tool to micromanipulate cells," Biophys. J. 1, 767-784 (2001).

86. J. E. Curtis, B. A. Koss, and D. G. Grier, "Dynamic holographic optical tweezers,” Opt. Commun. 207, 169-175 (2002).

87. D. Grier and Y. Roichman, "Holographic optical trapping," Appl. Opt. 45, 880-887 (2006).

88. R. Di Leonardo, F. Ianni, and G. Ruocco, “Computer generation of optimal holograms for optical trap arrays," Opt. Express 15, 1913-1922 (2007).

89. G. Spalding, J. Courtial, and R. D. Leonardo, chapter Holographic Optical Trapping in Structured Light and its Applications: An Introduction to Phase-Structured Beams and Nanoscale Optical Forces (Elsevier, Academic Press, 2008).

90. R. W. Bowman, G. Gibson, D. Carberry, L. Picco, M. Miles, and M. J. Padgett, "iTweezers: optical micromanipulation controlled by an Apple iPad," J. Opt. 13, 044002 (2011).

91. G. Gibson, L. Barron, F. Beck, G. Whyte, and M. Padgett, "Optically controlled grippers for manipulating micron-sized particles,” New. J. Phys. 9, 14 (2007)

92. G. Whyte, G. Gibson, J. Leach, M. Padgett, D. Robert, and M. Miles, “An optical trapped microhand for manipulating micron-sized objects," Opt. Express 14, 12497-12502 (2006).

93. C. Pacoret, R. Bowman, G. Gibson, S. Haliyo, D. Carberry, A. Bergander, S. Regnier, and M. Padgett, "Touching the microworld with force-feedback optical tweezers," Opt. Express 17, 10259-10264 (2009).

94. C. McDonald, M. McPherson, C. McDougall, and D. McGloin, "HoloHands: games console interface for controlling holographic optical manipulation," J. Opt. 15, 035708 (2013).

95. L. Shaw, D. Preece, and H. Rubinsztein-Dunlop, “Kinect the dots: 3D control of optical tweezers,” J. Opt. 15, 075303 (2013).

96. C. Muhiddin, D. Phillips, M. Miles, L. Picco, and D. Carberry, “Kinect 4 ... holographic optical tweezers ,” J. Opt. 15, 075302 (2013).

97. Z. Tomori, P. Keša, M. Nikorovič, J. Kaňka, P. Ják1, M. Šerý, S. Bernatová, E. V. sová, M. Antalík, and P. Zemánek, "Holographic raman tweezers controlled by multimodal natural user interface," J. Opt. 18, 015602:1-9 (2016).

98. J. Berthelot, S. S. Acimovic, M. L. Juan, M. P. Kreuzer, J. Renger, and R. Quidant, “Three-dimensional manipulation with scanning near-field optical nanotweezers," Nature Nanotech 9, 295-299 (2014).

99. M. Righini, A. S. Zelenina, C. Girard, and R. Quidant, "Parallel and selective trapping in a patterned plasmonic landscape," Nature Phys. 3, 477-480 (2007).

100. M. Righini, G. Volpe, C. Girard, D. Petrov, and R. Quidant, "Surface plasmon optical tweezers: Tunable optical manipulation in the femtonewton range," Phys. Rev. Lett. 100, 186804 (2008).

101. A. Grigorenko, N. Roberts, M. Dickinson, and Y. Zhang, "Nanometric optical tweezers based on nanostructured substrates," Nat. Photonics 2, 365-370 (2008).

102. M. M. Wang, E. Tu, D. E. Raymond, J. M. Yang, H. Zhang, N. Hagen, B. Dees, E. M. Mercer, A. H. Forster, I. Kariv, P. J. Marchand, and W. F. Butler, "Microfluidic sorting of mammalian cells by optical force switching," Nature Biotechnol. 23, 83-87 (2005).

103. P. T. Korda, M. B. Taylor, and D. G. Grier, "Kinetically locked-in colloidal transport in an array of optical tweezers," Phys. Rev. Lett. 89, 128301 (2002).

104. K. Dholakia, M. P. MacDonald, P. Zemánek, and T. Čižmár, "Cellular and colloidal separation using optical forces," Methods in Cell Biology 82, 467-495 (2007).

105. T. Čižmár, M. Šiler, M. Šerý, P. Zemánek, V. Garcés-Chávez, and K. Dholakia, “Optical sorting and detection of sub-micron objects in a motional standing wave,” Phys. Rev. B 74, 035105:1-6 (2006).

106. P. Jákl, T. Čižmár, M. Šerý, and P. Zemánek, "Static optical sorting in a laser interference field,” Appl. Phys. Lett. 92, 161110:1-3 (2008).

107. T. Čižmár, V. Garcés-Chávez, K. Dholakia, and P. Zemánek, “Optical conveyor belt for delivery of submicron objects," Appl. Phys. Lett. 86, 174101 (2005).

108. T. Čižmár, V. Kollárová, Z. Bouchal, and P. Zemánek, "Sub-micron particle organization by self-imaging of 
non-diffracting beams," New. J. Phys. 8, 43:1-23 (2006).

109. D. B. Ruffner and D. G. Grier, "Universal, strong and long-ranged trapping by optical conveyors," Opt. Express. 22, 26834-26843 (2014).

110. J. Chen, J. Ng, Z. Lin, and C. T. Chan, "Optical pulling force,” Nat. Photonics 5, 531-534 (2011).

111. A. Dogariu, S. Sukhov, and J. Saenz, "Optically induced 'negative forces,"” Nat. Photonics 7, 24-27 (2013).

112. A. Novitsky, C.-W. Qiu, and H. Wang, "Single Gradientless Light Beam Drags Particles as Tractor Beams," Phys. Rev. Lett. 107, 203601 (2011).

113. A. A. R. Neves and C. L. Cesar, "Analytical calculation of optical forces on spherical particles in optical tweezers: tutorial,” J. Opt. Soc. Am. B 36, 1525-1537 (2019).

114. A. Ashkin, "Forces of a single-beam gradient laser trap on a dielectric sphere in the ray optics regime," Biophys. J. 61, 569-582 (1992).

115. R. Gussgard, T. Lindmo, and I.Brevik, "Calculation of the trapping force in a strongly focused laser beam," J. Opt. Soc. Am. B 9, 1922-1929 (1992).

116. P. Chaumet and M. Nieto-Vesperinas, "Time-averaged total force on a dipolar sphere in an electromagnetic field," Opt. Lett. 25, 1065-1067 (2000).

117. C. F. Bohren and D. R. Huffman, Absorption and Scattering of Light by Small Particles (John Wiley \& Sons, New York, 1998).

118. S. Albaladejo, M. I. Marques, M. Laroche, and J. J. Saenz, "Scattering Forces from the Curl of the Spin Angular Momentum of a Light Field," Phys. Rev. Lett. 102, 113602 (2009).

119. L. Novotny and B. Hecht, Principles of nano-Optics (Cambridge University Press, Cambridge, 2006).

120. I. Iglesias and J. Sáenz, "Light spin forces in optical traps: comment on trapping metallic rayleigh particles with radial polarization,” Opt. Express 20, 2832-2834 (2015).

121. B. Draine, "The discrete-dipole approximation and its application to interstellar graphite grains," Astrophys J. 333, 848-872 (1988).

122. J. Kepler, De Cometis libelli tres (Augustae Vindelicorum, Augsburg, 1619).

123. C. R. McInnes, Solar Sailing: Technology, Dynamics and Mission Applications (Springer, London, 2013).

124. http://www.planetary.org/explore/projects/lightsail-solar-sailing/story-of-lightsail-part-2.html.

125. Y. Tsuda, O. Mori, R. Funase, H. Sawada, T. Yamamoto, T. Saiki, T. Endo, and J. Kawaguchi, "Flight status of IKAROS deep space solar sail demonstrator," Acta Astronautica 69, 833 - 840 (2011).

126. http://www.nasa.gov/mission_pages/smallsats/nanosaild.html.

127. W. J. Borucki, D. Koch, G. Basri, N. Batalha, T. Brown, D. Caldwell, J. Caldwell, J. Christensen-Dalsgaard, W. D. Cochran, E. DeVore, E. W. Dunham, A. K. Dupree, T. N. Gautier, III, J. C. Geary, R. Gilliland, A. Gould, S. B. Howell, J. M. Jenkins, Y. Kondo, D. W. Latham, G. W. Marcy, S. Meibom, H. Kjeldsen, J. J. Lissauer, D. G. Monet, D. Morrison, D. Sasselov, J. Tarter, A. Boss, D. Brownlee, T. Owen, D. Buzasi, D. Charbonneau, L. Doyle, J. Fortney, E. B. Ford, M. J. Holman, S. Seager, J. H. Steffen, W. F. Welsh, J. Rowe, H. Anderson, L. Buchhave, D. Ciardi, L. Walkowicz, W. Sherry, E. Horch, H. Isaacson, M. E. Everett, D. Fischer, G. Torres, J. A. Johnson, M. Endl, P. MacQueen, S. T. Bryson, J. Dotson, M. Haas, J. Kolodziejczak, J. Van Cleve, H. Chandrasekaran, J. D. Twicken, E. V. Quintana, B. D. Clarke, C. Allen, J. Li, H. Wu, P. Tenenbaum, E. Verner, F. Bruhweiler, J. Barnes, and A. Prsa, "Kepler Planet-Detection Mission: Introduction and First Results," Science 327, 977-980 (2010).

128. https://www.nasa.gov/kepler/missiontimeline.

129. https://breakthroughinitiatives.org/initiative/3.

130. H. A. Atwater, A. R. Davoyan, O. Ilic, D. Jariwala, M. C. Sherrott, C. M. Went, W. S. Whitney, and J. Wong, "Materials challenges for the Starshot lightsail," Nature Mater. 17, 861-867 (2018).

131. O. Ilic and H. A. Atwater, "Self-stabilizing photonic levitation and propulsion of nanostructured macroscopic objects," Nat. Photonics 13, 289-295 (2019).

132. A. E. Siegman, Lasers. (Univ. Sci. Books, Sausalito, California, 1986).

133. Y. Harada and T. Asakura, "Radiation forces ona dielectric sphere in the Rayleigh scattering regime," Opt. Commun. 124, 529-541 (1996).

134. P. Zemánek, A. Jonáš, L. Šrámek, and M. Liška, "Optical trapping of Rayleigh particles using a Gaussian standing wave," Opt. Commun. 151, 273-285 (1998).

135. A. S. Urban, A. A. Lutich, F. D. Stefani, and J. Feldmann, "Laser printing single gold nanoparticles," Nano Lett. 10, 4794-4798 (2010).

136. O. M. Maragò, P. H. Jones, P. G. Gucciardi, G. Volpe, and A. C. Ferrari, "Optical trapping and manipulation of nanostructures," Nat. Nanotechnol. 8, 807-819 (2013).

137. A. S. Urban, S. Carretero-Palacios, A. A. Lutich, T. Lohmueller, J. Feldmann, and F. Jaeckel, "Optical trapping and manipulation of plasmonic nanoparticles: fundamentals, applications, and perspectives," Nanoscale 6, 4458-4474 (2014).

138. A. S. Urban, M. Fedoruk, S. Nedev, A. Lutich, T. Lohmueller, and J. Feldmann, "Shrink-to-fit plasmonic nanostructures," Adv. Optical Mater. 1, 123-127 (2013).

139. Y. Bao, Z. Yan, and N. F. Scherer, "Optical printing of electrodynamically coupled metallic nanoparticle arrays," J. Phys. Chem. C 118, 19315-19321 (2014).

140. M. M. Burns, J.-M. Fournier, and J. A. Golovchenko, "Optical Binding," Phys. Rev. Lett. 63, 1233-1236 (1989).

141. K. Dholakia and P. Zemánek, “Gripped by light: Optical binding,” Rev. Mod. Phys. 82, 1767-1791 (2010). 
142. V. Demergis and E.-L. Florin, "Ultrastrong optical binding of metallic nanoparticles," Nano Lett. 12, 5756-5760 (2012).

143. M. J. Guffey and N. F. Scherer, "All-optical patterning of Au nanoparticles on surfaces using optical traps," Nano Lett. 10, 4302-4308 (2010).

144. Z. Yan, S. K. Gray, and N. F. Scherer, "Potential energy surfaces and reaction pathways for light-mediated self-organization of metal nanoparticle clusters," Nat. Commun. 5 (2014).

145. L. Chvátal, O. Brzobohatý, and P. Zemánek, "Binding of a pair of Au nanoparticles in a wide Gaussian standing wave," Opt. Rev. 22, 157-161 (2015).

146. J. Gargiulo, S. Cerrota, E. Cortes, I. L. Violi, and F. D. Stefani, “Connecting Metallic Nanoparticles by Optical Printing," Nano Lett. 16, 1224-1229 (2016).

147. R. Piazza and A. Parola, "Thermophoresis in colloidal suspensions," J. Phys.-Condes. Matter 20, 153102 (2008).

148. J. Do, M. Fedoruk, F. Jaeckel, and J. Feldmann, "Two-color laser printing of individual gold nanorods," Nano Lett. 13, 4164-4168 (2013).

149. J. Trojek, L. Chvátal, and P. Zemánek, "Optical alignment and confinement of an ellipsoidal nanorod in optical tweezers: A theoretical study,” J. Opt. Soc. Am. A 29, 1224-1236 (2012).

150. O. Brzobohatý, M. Šiler, J. Trojek, L. Chvátal, V. Karásek, A. Paták, Z. Pokorná, F. Mika, and P. Zemánek, "Three-dimensional optical trapping of a plasmonic nanoparticle using low numerical aperture optical tweezers," Sci. Rep. 5, 8106 (2015).

151. M. J. Guffey, R. L. Miller, S. K. Gray, and N. F. Scherer, "Plasmon-driven selective deposition of Au bipyramidal nanoparticles," Nano Lett. 11, 4058-4066 (2011).

152. M. Ana Huergo, C. M. Maier, M. Federico Castez, C. Vericat, S. Nedev, R. C. Salvarezza, A. S. Urban, and J. Feldmann, "Optical nanoparticle sorting elucidates synthesis of plasmonic nanotriangles," ACS Nano 10, 3614-3621 (2016).

153. S. Bernatová, M. G. Donato, J. Ježek, Z. Pilát, O. Samek, A. Magazzu, O. M. Maragó, P. Zemánek, and P. G. Gucciardi, "Wavelength-dependent optical force aggregation of gold nanorods for SERS in a microfluidic chip," J. Phys. Chem. C 123, 5608-5615 (2019).

154. A. Babynina, M. Fedoruk, P. Kuehler, A. Meledin, M. Doeblinger, and T. Lohmueller, "Bending gold nanorods with light," Nano Lett. 16, 6485-6490 (2016).

155. C. Zaza, I. Violo, J. Gargiulo, G. Chiarelli, L. Schumacher, J. Jakobi, J. Olomos-Trigo, E. Cortes, M. Konig, S. Barcikowski, S. Schlucker, J. Sáenz, S. Maier, and F. Stefani, "Size-selective optical printing of silicon nanoparticles through their dipolar magnetic resonance," ACS Photonics, (2019).

156. A. I. Kuznetsov, A. E. Miroshnichenko, M. L. Brongersma, Y. S. Kivshar, and B. Luk'yanchuk, "Optically resonant dielectric nanostructures," Science 354 (2016).

157. I. Staude and J. Schilling, "Metamaterial-inspired silicon nanophotonics," Nat. Photonics 11, 274-284 (2017).

158. S. Jahani and Z. Jacob, “All-dielectric metamaterials," Nat. Nanotech. 11, 23-36 (2016).

159. M. Li, T. Lohmueller, and J. Feldmann, "Optical injection of gold nanoparticles into living cells," Nano Lett. 15, 770-775 (2015).

160. C. M. Maier, M. A. Huergo, S. Milosevic, C. Pernpeintner, M. Li, D. P. Singh, D. Walker, P. Fischer, J. Feldmann, and T. Lohmueller, "Optical and thermophoretic control of Janus nanopen injection into living cells," Nano Lett. 18, 7935-7941 (2018).

161. S. Nedev, A. S. Urban, A. A. Lutich, and J. Feldmann, "Optical Force Stamping Lithography," Nano Lett. 11, 5066-5070 (2011).

162. T. Čižmár, M. Šiler, and P. Zemánek, “An optical nanotrap array movable over a milimetre range,” Appl. Phys. B 84, 197-203 (2006).

163. J. Durnin, J. J. Miceli, and J. Eberly, "Diffraction-free beams," Phys. Rev. Lett. 58, 1499-1501 (1987).

164. Z. Bouchal and M. Olivík, "Non-diffractive vector Bessel beams," J. Mod. Optics 42, 1555-1566 (1995).

165. M. R. Lapointe, "Review of non-diffracting Bessel beam experiments," Opt. Laser Technol. 24, 315-321 (1992).

166. D. McGloin and K. Dholakia, "Bessel beams: diffraction in a new light," Contemp. Phys. 46, 15-28 (2005).

167. T. Čižmár and K. Dholakia, "Tunable Bessel light modes: engineering the axial propagation," Opt. Express 17, 15558-15570 (2009).

168. G. Indebetouw, "Nondiffracting optical fields: some remarks in their analysis and synthesis," J. Opt. Soc. Am. A 6, $150-152$ (1989).

169. V. Jarutis, R. Paškauskas, and A. Stabinis, "Focusing of Laguerre-Gaussian beams by axicon," Opt. Commun. 184 1-4, 105-112 (2000).

170. O. Brzobohatý, T. Čižmár, and P. Zemánek, "High quality quasi-Bessel beam generated by round-tip axicon,” Opt. Express 16, 12688-12700 (2008).

171. J. Durnin, J. J. Miceli, and J. Eberly, “Comparison of Bessel and Gaussian beams,” Opt. Lett. 13, 79-80 (1988).

172. Z. Bouchal, J. Wagner, and M. Chlup, "Self-reconstruction of a distorted nondiffracting beam," Opt. Commun. 151, 207-211 (1998).

173. V. Garcés-Chávez, D. McGloin, H. Melville, W. Sibbett, and K. Dholakia, "Simultaneous micromanipulation in multiple planes using a self- reconstructing light beam," Nature 419, 145-147 (2002).

174. H. Little, C. B. V. Garcés-Chávez, W. Sibbett, and K. Dholakia, "Optical guiding of microscopic particles in femtosecond and continuous wave Bessel light beams,” Opt. Express 12, 2560-2565 (2004). 
175. P. Fischer, A. E. Carruthers, K. Volke-Sepúlveda, E. M. Wright, C. Brown, W. Sibbett, and K. Dholakia, "Enhanced optical guiding of colloidal particles using a supercontinuum light source," Opt. Express 14, 5792-5802 (2006).

176. P. Fischer, C. T. A. Brown, J. E. Morris, C. López-Mariscal, E. M. Wright, W. Sibbett, and K. Dholakia, "White light propagation invariant beams," Opt. Express 13, 6657-6666 (2005).

177. R. Yang and R. Li, "Optical force exerted on a Rayleigh particle by a vector arbitrary-order Bessel beam," J. Quant. Spectrosc. Radiat. Transf. 178, 230-243 (2016).

178. G. Milne, K. Dholakia, D. McGloin, K. Volke-Sepúlveda, and P. Zemánek, "Transverse particle dynamics in a Bessel beam,” Opt. Express 15, 13972-13987 (2007).

179. M. Šiler, P. Jákl, O. Brzobohatý, and P. Zemánek, "Optical forces induced behavior of a particle in a non-diffracting vortex beam," Opt. Express 20, 24304-24319 (2012).

180. K. Gahagan and G. Swartzlander, "Optical vortex trapping of particles," Opt. Lett. 21, 827-829 (1996).

181. K. Gahagan and G. Swartzlander, "Trapping of low-index microparticles in an optical vortex," J. Opt. Soc. Am. B 15, 524-534 (1998).

182. J. Arlt, T. Hitomi, and K. Dholakia, "Atom guiding along Laguerre-Gaussian and Bessel light beams," Appl. Phys. B 71, 549-556 (2000).

183. J. Arlt, K. Dholakia, J. Soneson, and E. M. Wright, "Optical dipole traps and atomic waveguides based on Bessel light beams," Phys. Rev. A 63, 063602 (2001).

184. J. E. Morris, T. Čižmár, H. I. C. Dalgarno, R. F. Marchington, F. J. Gunn-Moore, and K. Dholakia, "Realization of curved Bessel beams: propagation around obstructions," J. Opt. 12, 124002 (2010).

185. V. Jarutis, A. Matijošius, P. Di Trapani, and A. Piskarskas, "Spiraling zero-order Bessel beam," Opt. Lett. 14, 2129-2131 (2009).

186. J. Zhao, I. D. Chremmos, Z. Zhang, Y. Hu, D. Song, P. Zhang, N. K. Efremidis, and Z. Chen, "Specially shaped Bessel-like self-accelerating beams along predesigned trajectories," Sci. Bull. 60, 1157-1169 (2015).

187. S.-H. Lee, Y. Roichman, and D. Grier, “Optical solenoid beams,” Opt. Express 18, 6988-6993 (2010).

188. V. Daria, D. Palima, and J. Glückstad, "Optical twists in phase and amplitude," Opt. Express 19, 476-481 (2011)

189. M. Berry and N. Balazz, "Nonspreading wave packets," Am. J. Phys. 47, 264-267 (1979).

190. G. A. Siviloglou and D. N. Christodoulides, "Accelerating finite energy airy beams," Opt. Lett. 32, 979-981 (2007).

191. P. Zhang, Y. Hu, T. Li, D. Cannan, X. Yin, R. Morandotti, Z. Chen, and X. Zhang, "Nonparaxial Mathieu and Weber Accelerating Beams," Phys. Rev. Lett. 109, 193901 (2012).

192. S. Yan, M. Li, B. Yao, X. Yu, M. Lei, D. Dan, Y. Yang, J. Min, and T. Peng, “Accelerating nondiffracting beams," Phys. Lett. A 379, 983-987 (2015).

193. M. A. Bandres and B. Rodríguez-Lara, "Nondiffracting accelerating waves: Weber waves and parabolic momentum," New J. Phys. 15, 013054 (2013).

194. T. Čižmár, V. Kollárová, X. Tsampoula, F. Gunn-Moore, W. Sibbett, and K. Dholakia, "Generation of multiple Bessel beams for a biophotonic workstation," Opt. Express 16, 14024-14035 (2008).

195. G. A. Siviloglou, J. Broky, A. Dogariu, and D. N. Christodoulides, "Observation of accelerating airy beams," Phys. Rev. Lett. 99, 213901 (2007).

196. G. A. Siviloglou, J. Broky, A. Dogariu, and D. N. Christodoulides, "Ballistic dynamics of airy beams," Opt. Lett. 33, 207-209 (2008).

197. J. Morris, M. Mazilu, J. Baumgartl, T. Čižmár, and K. Dholakia, "Propagation characteristics of Airy beams: dependence upon spatial coherence and wavelength," Opt. Express 17, 13236-13245 (2009).

198. J. Baumgartl, M. Mazilu, and K. Dholakia, "Optically mediated particle clearing using Airy wavepackets," Nat. Photonics 2, 675-678 (2008).

199. J. Baumgartl, G. Hannappel, D. Stevenson, D. Day, M. Gu, and K. Dholakia, "Optical redistribution of microparticles and cells between microwells," Lab Chip 9, 1334-1336 (2009).

200. J. Baumgartl, T. Čižmár, M. Mazilu, V. C. Chan, A. E. Carruthers, B. A. Capron, W. McNeely, E. M. Wright, and K. Dholakia, "Optical path clearing and enhanced transmission through colloidal suspensions," Opt. Express 18, 17130-17140 (2010).

201. X. Yu, R. Li, S. Yan, B. Yao, P. Gao, G. Han, and M. Lei, "Experimental demonstration of 3D accelerating beam arrays," Appl. Opt. 55, 3090-3095 (2016).

202. P. Zhang, J. Prakash, Z. Zhang, M. S. Mills, N. K. Efremidis, D. N. Christodoulides, and Z. Chen, "Trapping and guiding microparticles with morphing autofocusing airy beams,” Opt. Lett. 36, 2883-2885 (2011).

203. I. Kaminer, R. Bekenstein, J. Nemirovsky, and M. Segev, "Nondiffracting accelerating wave packets of MaxwellâĂŹs equations," Phys. Rev. Lett. 108, 163901 (2012).

204. M. A. Bandres, M. A. Alonso, I. Kaminer, and M. Segev, "Three-dimensional accelerating electromagnetic waves," Opt. Express 21, 13917-13929 (2013).

205. C. López-Mariscal, J. C. Gutiérrez-Vega, G. Milne, and K. Dholakia, "Orbital angular momentum transfer in helical Mathieu beams," Opt. Express 14, 4182-4187 (2006).

206. A. Mathis, F. Courvoisier, R. Giust, L. Furfaro, M. Jacquot, L. Froehly, and J. M. Dudley, "Arbitrary nonparaxial accelerating periodic beams and spherical shaping of light," Opt. Lett. 38, 2218-2220 (2013).

207. A. Ruelas, J. A. Davis, I. Moreno, D. M. Cottrell, and M. A. Bandres, "Accelerating light beams with arbitrarily transverse shapes," Opt. Express 22, 3490-3500 (2014).

208. R. Schley, I. Kaminer, E. Greenfield, R. Bekenstein, Y. Lumer, and M. Segev, "Loss-proof self-accelerating beams 
and their use in non-paraxial manipulation of particles' trajectories," Nat. Commun. 5, 5189 (2014).

209. S. Sukhov and A. Dogariu, "On the concept of "tractor beams"," Opt. Lett. 35, 3847-3849 (2010).

210. J. Saenz, "Laser tractor beams," Nat. Photonics 5, 514-515 (2011).

211. S. Sukhov and A. Dogariu, "Negative Nonconservative Forces: Optical "Tractor Beams" for Arbitrary Objects," Phys. Rev. Lett. 107, 203602 (2011).

212. P. Forgács, A. Lukács, and T. Romańczukiewicz, "Plane waves as tractor beams," Phys. Rev. D 88, 125007 (2013).

213. A. Akbarzadeh, M. Danesh, C.-W. Qiu, and A. J. Danner, "Tracing optical force fields within graded-index media," New J. Phys. 16, 053035 (2014).

214. J. Ng, Z. F. Lin, C. T. Chan, and P. Sheng, "Photonic clusters formed by dielectric microspheres: Numerical simulations," Phys. Rev. B 72, 085130 (2005).

215. A. Akbarzadeh, J. A. Crosse, M. Danesh, C.-W. Qiu, A. J. Danner, and C. M. Soukoulis, "Interplay of Optical Force and Ray-Optic Behavior between Luneburg Lenses," ACS Phot. 2, 1384-1390 (2015).

216. J. Damková, L. Chvátal, J. Ježek, J. Oulehla, O. Brzobohatý, and P. Zemánek, "Enhancement of the 'tractor-beam' pulling force on an optically bound structure," Light Sci. Appl. 7, 17135 (2018).

217. N. Wang, J. Chen, S. Liu, and Z. Lin, "Dynamical and phase-diagram study on stable optical pulling force in bessel beams," Phys. Rev. A 87, 063812 (2013).

218. A. Novitsky, C.-W. Qiu, and A. Lavrinenko, "Material-Independent and Size-Independent Tractor Beams for Dipole Objects,” Phys. Rev. Lett. 109, 053815 (2012).

219. D. Gao, A. Novitsky, T. Zhang, F. C. Cheong, L. Gao, C. T. Lim, B. Luk'yanchuk, and C.-W. Qiu, "Unveiling the correlation between non-diffracting tractor beam and its singularity in Poynting vector," Laser Photon. Rev. 9, 75-82 (2015).

220. X. Li, J. Chen, Z. Lin, and J. Ng, “Optical pulling at macroscopic distances,” Sci. Adv. 5, eaau7814 (2019).

221. P. L. Marston, "Axial radiation force of a Bessel beam on a sphere and direction reversal of the force," J. Acoust. Soc. Am. 120, 3518-3524 (2006).

222. F. Mitri, "Negative axial radiation force on a fluid and elastic spheres illuminated by a high-order Bessel beam of progressive waves," J. Phys. A 42, 245202 (2009).

223. P. L. Marston, "Negative axial radiation forces on solid spheres and shells in a Bessel beam ," J. Acoust. Soc. Am. 122, 3162-3165 (2007).

224. P. L. Marston, "Scattering of a Bessel beam by a sphere," J. Acoust. Soc. Am. 121, 753-758 (2007).

225. A. Gorlach, G. M.A., A. Lavrinenko, and A. Novitsky, "Matter-wave tractor beam," Phys. Rev. Lett. 118, 180401 (2017).

226. M. Nieto-Vesperinas, J. J. Saenz, R. Gomez-Medina, and L. Chantada, "Optical forces on small magnetodielectric particles," Opt. Express 18, 11428-11443 (2010).

227. R. Gómez-Medina, B. García-Cámara, I. Suárez-Lacalle, L. Froufe-Pérez, F. González, F. Moreno, M. NietoVesperinas, and J. Sáenz, "Electric and magnetic optical response of dielectric nanospheres: Optical forces and scattering anisotropy," Photonics Nanostruct. Fundam. Appl. 10, 345-352 (2012).

228. P. Chaumet and A. Rahmani, "Electromagnetic force and torque on magnetic and negative-index scatteres," Opt. Express 17, 2224-2234 (2009).

229. T. Kudo and H. Ishihara, "Proposed Nonlinear Resonance Laser Technique for Manipulating Nanoparticles," Phys. Rev. Lett. 109, 087402 (2012).

230. A. Mizrahi and Y. Fainman, "Negative radiation pressure on gain medium structures," Opt. Lett. 35, 3405-3407 (2010).

231. A. Novitsky, W. Ding, M. Wang, D. Gao, A. V. Lavrinenko, and C.-W. Qiu, "Pulling cylindrical particles using a soft-nonparaxial tractor beam," Sci. Rep. 7, 652 (2017).

232. A. Novitsky and C.-W. Qiu, "Pulling extremely anisotropic lossy particles using light without intensity gradient," Phys. Rev. A 90, 053815 (2014).

233. K. Ding, J. Ng, L. Zhou, and C. T. Chan, "Realization of optical pulling forces using chirality,” Phys. Rev. A 89, 063825 (2014).

234. A. Constable, J. Kim, J. Mervis, F. Zarinetchi, and M. Prentiss, "Demonstration of a fiber-optical light-force trap," Opt. Lett. 18, 1867-1869 (1993).

235. T. Čižmár and K. Dholakia, "Exploiting multimode waveguides for pure fibre-based imaging,” Nat. Commun. 3, 1027:1-9 (2012).

236. T. Čižmár and K. Dholakia, "Shaping the light transmission through a multimode optical fibre: complex transformation analysis and applications in biophotonics," Opt. Express 19, 18871-18884 (2011).

237. S. Bianchi and R. Di Leonardo, "A multi-mode fiber probe for holographic micromanipulation and microscopy," Lab Chip 12, 635-639 (2012).

238. D. M. Gherardi, A. E. Carruthers, T. Čižmár, E. M. Wright, and K. Dholakia, “A dual beam photonic crystal fibre trap for microscopic particles," Appl. Phys. Lett. 93, 041110 (2008).

239. O. G. Helles $\varnothing$, P. Løvhaugen, S. A.Z., W. J.S., and A. B.S., "Surface transport and stable trapping of particles and cells by an optical waveguide loop," Lab Chip 12, 3436-3440 (2012).

240. S. J. Cran-McGreehin, T. F. Krauss, and K. Dholakia, "Integrated monolithic optical manipulation,” Lab Chip 6, 1122-1124 (2006).

241. G. Roosen and C. Imbert, "Optical levitation by means of two horizontal laser beams: a theoretical and experimental 
study," Phys. Lett. 59A, 6-8 (1976).

242. G. Roosen, "A theoretical and experimental study of the stable equilibrium positions of spheres levitated by two horizontal laser beams," Opt. Commun. 21, 189-194 (1977).

243. G. Roosen and S. Slansky, "Influence of the beam divergence on the exerted force on a sphere by a laser beam and required conditions for stable optical levitation," Opt. Commun. 29, 341-346 (1979).

244. W. Singer, M. Frick, S. Bernet, and M. Ritsch-Marte, "Self-organized array of regularly spaced microbeads in a fiber-optical trap," J. Opt. Soc. Am. B 20, 1568-1574 (2003).

245. S. A. Tatarkova, A. E. Carruthers, and K. Dholakia, "One-Dimensional Optically Bound Arrays of Microscopic Particles," Phys. Rev. Lett. 89, 283901 (2002).

246. M. M. Burns, J.-M. Fournier, and J. A. Golovchenko, "Optical matter: Crystallization and binding in intense optical fields," Science 249, 749 (1990).

247. N. K. Metzger, E. M. Wright, W. Sibbett, and K. Dholakia, "Visualization of optical binding of microparticles using a femtosecond fiber optical trap," Opt. Express 14, 3677-3687 (2006).

248. N. K. Metzger, R. F. Marchington, M. Mazilu, R. L. Smith, K. Dholakia, and E. M. Wright, "Measurement of the restoring forces acting on two optically bound particles from normal mode correlations," Phys. Rev. Lett. 98, 068102 (2007).

249. N. K. Metzger, E. M. Wright, and K. Dholakia, "Theory and simulation of the bistable behaviour of optically bound particles in the Mie size regime," New. J. Phys. 8, 139 (2006).

250. N. K. Metzger, K. Dholakia, and E. M. Wright, "Observation of Bistability and Hysteresis in Optical Binding of Two Dielectric Spheres," Phys. Rev. Lett. 96, 068102 (2006).

251. V. Karásek, K. Dholakia, and P. Zemánek, "Analysis of optical binding in one dimension,” Appl. Phys. B 84, 149-156 (2006).

252. V. Karásek, T. Čižmár, O. Brzobohatý, P. Zemánek, V. Garcés-Chávez, and K. Dholakia, "Long-range onedimensional longitudinal optical binding," Phys. Rev. Lett. 101, 143601 (2008).

253. V. Karásek and P. Zemánek, "Analytical description of longitudinal optical binding of two spherical nanoparticles," J. Opt. A: Pure Appl. Opt. 9, S215-S220 (2007).

254. O. Brzobohatý, V. Karásek, T. Čižmár, and P. Zemánek, "Dynamic size tuning of multidimensional optically bound matter," Appl. Phys. Lett. 99, 101105 (2011).

255. O. Brzobohatý, L. Chvátal, A. Jonáš, M. Šiler, J. Kaňka, J. Ježek, and P. Zemánek, “Tunable soft-matter optofluidic waveguides assembled by light," ACS Phot. 6, 403-410 (2019).

256. M. Guillon, O. Moine, and B. Stout, "Longitudinal optical binding of high optical contrast microdroplets in air," Phys. Rev. Lett. 96, 143902 (2006).

257. O. Brzobohatý, M. Šiler, J. Ježek, P. Jákl, and P. Zemánek, "Optical manipulation of aerosol droplets using a holographic dual and single beam trap,” Opt. Lett. 38, 4601-4604 (2013).

258. T. Li, S. Kheifets, and M. G. Raizen, "Millikelvin cooling of an optically trapped microsphere in vacuum," Nature Phys 7, 527-530 (2011).

259. S. Chu, "The manipulation of neutral particles," Rev. Mod. Phys. 70, 685-706 (1998).

260. B. E. A. Saleh and M. C. Teich, Fundamentals of Photonics (John Wiley \& Sons., Hoboken, New Jersey, 2007).

261. P. Zemánek, A. Jonáš, P. Jákl, M. Šerý, J. Ježek, and M. Liška, "Theoretical comparison of optical traps created by standing wave and single beam," Opt. Commun. 220, 401-412 (2003).

262. P. Zemánek, A. Jonáš, L. Šrámek, and M. Liška, "Optical trapping of nanoparticles and microparticles using Gaussian standing wave." Opt. Lett. 24, 1448-1450 (1999).

263. A. Jonáš, P. Zemánek, and E. L. Florin, "Single beam trapping in front of reflective surfaces," Opt. Lett. 26, $1466-1468$ (2001).

264. P. Zemánek, A. Jonáš, and M. Liška, "Simplified description of optical forces acting on a nanoparticle in the Gaussian standing wave," J. Opt. Soc. Am. A 19, 1025-1034 (2002).

265. G. Thalhammer, R. Steiger, S. Bernet, and M. Ritsch-Marte, "Optical macro-tweezers: trapping of highly motile micro-organisms," J. Opt. 13, 044024 (2011).

266. S. Zwick, T. Haist, Y. Miyamoto, L. He, M. Warber, A. Hermerschmidt, and W. Osten, "Holographic twin traps," J. Opt. A: Pure Appl. Opt. 11, 034011 (2009).

267. I. Ricardez-Vargas and K. Volke-Sepúlveda, "Experimental generation and dynamical reconfiguration of different circular optical lattices for applications in atom trapping," J. Opt. Soc. Am. B 27, 948-955 (2010).

268. Y. Miroshnychenko, D. Schrader, S. Kuhr, W. Alt, I. Dotsenko, M. Khudaverdyan, A. Rauschenbeutel, and D. Meschede, "Continued imaging of the transport of a single neutral atom," Opt. Express 11, 3498-3502 (2003).

269. I. Dotsenko, W. Alt, M. Khudaverdyan, S. Kuhr, D. Meschede, Y. Miroshnychenko, D. Schrader, and A. Rauschenbeutel, "Submicrometer position control of single trapped neutral atoms," Phys. Rev. Lett. 95, 033002 (2005).

270. J. Wagner and Z. Bouchal, "Experimental realization of self-reconstruction of the 2D aperiodic objects," Opt. Commun. 176, 309-311 (2000).

271. P. Reimann, "Brownian Motors: noisy transport far from equilibrium," Phys. Rep. 361, 57-265 (2002).

272. M. Šiler, T. Čižmár, A. Jonáš, and P. Zemánek, "Surface delivery of a single nanoparticle under moving evanescent standing-wave illumination," New. J. Phys. 10, 113010:1-16 (2008).

273. S. Kuhr, W. Alt, D. Schrader, M. Müller, V. Gomer, and D. Meschede, "Deterministic Delivery of a Single Atom," Science 293, 278-280 (2001). 
274. S. Kuhr, W. Alt, D. Schrader, I. Dotsenko, Y. Miroshnychenko, W. Rosenfeld, M. Khudaverdyan, V. Gomer, A. Rauschenbeutel, and D. Meschede, "Coherence properties and quantum state transportation in an optical conveyor belt," Phys. Rev. Lett. 91, 213002 (2003).

275. S. Schmid, G. Thalhammer, K. Winkler, F. Lang, and J. H. Denschlag, "Long distance transport of ultracold atoms using a 1D optical lattice,” New. J. Phys. 8, 159 (2006).

276. A. Ashkin, J. M. Dziedzic, and T. Yamane, "Optical trapping and manipulation of single cells using infrared laser beams," Nature 330, 769-771 (1987).

277. K. Sasaki, M. Koshioka, H. Misawa, N. Kitamura, and H. Masuhara, "Pattern formation and flow control of fine particles by laser-scanning micromanipulation,” Opt. Lett. 16, 1463-1465 (1991).

278. C. Mio, T. Gong, A. Terray, and D. W. M. Marr, "Design of a scanning laser optical trap for multiparticle manipulation,” Rev. Sci. Instrum. 71, 2196-2200 (2000).

279. K. Visscher, S. P. Gross, and S. M. Block, "Construction of multiple-beam optical traps with nanometer-resolution position sensing," IEEE J. Sel. Top. Quantum Electron. 2, 1066-1076 (1996).

280. M. T. Valentine, N. R. Guydosh, B. Gutierrez-Medina, A. N. Fehr, J. O. Andreasson, and S. M. Block, "Precision steering of an optical trap by electro-optic deflection," Opt. Lett. 33, 599-601 (2008).

281. E. Fällman and O. Axner, "Design for fully steerable dual-trap optical tweezers," Appl. Opt. 36, 2107-2113 (1997).

282. M. Ozcan, C. Onal, and A. Akatay, "A compact, automated and long working distance optical tweezer system," J. Mod. Opt. 53, 357-364 (2006).

283. M. Reicherter, T. Haist, E. U. Wagemann, and H. J. Tiziani, "Optical particle trapping with computer-generated holograms written on a liquid-crystal display,” Opt. Lett. 24, 608-610 (1999).

284. G. M. Gibson, R. W. Bowman, A. Linnenberger, M. Dienerowitz, D. B. Phillips, D. M. Carberry, M. J. Miles, and M. J. Padgett, “A compact holographic optical tweezers instrument,” Rev. Sci. Instrum. 83, 113107 (2012).

285. T. Čižmár, M. Mazilu, and K. Dholakia, "In situ wavefront correction and its application to micromanipulation," Nat. Photonics 4, 388-394 (2010).

286. C. Basdogan, A. Kiraz, I. Bukusoglu, A. Varol, and S. Doğanay, "Haptic guidance for improved task performance in steering microparticles with optical tweezers," Opt. Express 15, 11616-11621 (2007).

287. J. A. Grieve, A. Ulcinas, S. Subramanian, G. M. Gibson, M. J. Padgett, D. M. Carberry, and M. J. Miles, "Hands-on with optical tweezers: a multitouch interface for holographic optical trapping," Opt. Express 17, 3595-3602 (2009).

288. E. Ferrari, V. Emiliani, D. Cojoc, V. Garbin, M. Zahid, C. Durieux, M. Coppey-Moisan, and E. Di Fabrizio, "Biological samples micro-manipulation by means of optical tweezers," Microelectr. Eng. 78-79, 575-581 (2005).

289. Y. Roichman, B. Sun, Y. Roichman, J. Amato-Grill, and D. G. Grier, "Optical Forces Arising from Phase Gradients," Phys. Rev. Lett. 100, 013602:1-4 (2008).

290. A. Jesacher, C. Maurer, A. Schwaighofer, S. Bernet, and M. Ritsch-Marte, "Full phase and amplitude control of holographic optical tweezers with high efficiency," Opt. Express 16, 4479-4486 (2008).

291. A. Ashkin and J. M. Dziedzic, "Optical trapping and manipulation of viruses and bacteria," Science 235, 1517-1520 (1987).

292. P. L. Johansen, F. Fenaroli, L. Evensen, G. Griffiths, and G. Koster, "Optical micromanipulation of nanoparticles and cells inside living zebrafish," Nat. Commun. 7, 10974 (2016).

293. H. Zhang and K.-K. Liu, "Optical tweezers for single cells," J. Royal Soc. Interface. 5, 671-690 (2008).

294. K. Schutze, H. Posl, and G. Lahr, "Laser micromanipulation systems as universal tools in cellular and molecular biology and in medicine,” Cell. Molec. Biol. 44, 735-746 (1998).

295. D. Gao, W. Ding, M. Nieto-Vesperinas, X. Ding, M. Rahman, T. Zhang, C. Lim, and C.-W. Qiu, "Optical manipulation from the microscale to the nanoscale: fundamentals, advances and prospects," Light Sci. Appl. 6 , e17039 (2017).

296. C. Hawes, A. Osterrieder, I. A. Sparkes, and T. Ketelaar, "Optical tweezers for the micromanipulation of plant cytoplasm and organelles," Curr. Opin. Plant Biol. 13, 731-735 (2010).

297. K. Norregaard, R. Metzler, C. M. Ritter, K. Berg-Sorensen, and L. B. Oddershede, "Manipulation and motion of organelles and single molecules in living cells," Chem. Rev. 117, 4342-4375 (2017).

298. T. L. Gustavson, A. P. Chikkatur, A. E. Leanhardt, A. Görlitz, S. Gupta, D. E. Pritchard, and W. Ketterle, "Transport of Bose-Einstein condensates with optical tweezers,” Phys. Rev. Lett. 88, 0204011-0204014 (2002).

299. A. Chikkatur, Y. Shin, A. Leanhardt, D. Kielpinski, E. Tsikata, T. Gustavson, D. Pritchard, and W. Ketterle, "A continuous source of Bose-Einstein condensed atoms," Science 296, 2193-2195 (2002).

300. W. D. Phillips, "Laser cooling and trapping of neutral atoms," Rev. Mod. Phys. 70, 721-741 (1998).

301. C. N. Cohen-Tannoudji, “Manipulating atoms with photons,” Rev. Mod. Phys. 70, 707-719 (1998).

302. V. Balykin, V. Minogin, and V. Letokhov, "Electromagnetic trapping of cold atoms," Rep. Prog. Phys. 63, 1429-1510 (2000).

303. J. Gieseler, B. Deutsch, R. Quidant, and L. Novotny, "Subkelvin parametric feedback cooling of a laser-trapped nanoparticle," Phys. Rev. Lett. 109 (2012).

304. P. Mestres, J. Berthelot, M. Spasenovic, J. Gieseler, L. Novotny, and R. Quidant, "Cooling and manipulation of a levitated nanoparticle with an optical fiber trap," Appl. Phys. Lett. 107 (2015).

305. I. T. Leite, S. Turtaev, X. Jiang, M. Šiler, A. Cuschieri, P. S. J. Russell, and T. Čižmár, "Three-dimensional holographic optical manipulation through a high-numerical-aperture soft-glass multimode fibre," Nat. Photonics 12, 33-39 (2018). 
306. C. Monat, P. Domachuk, and B. J. Eggleton, "Integrated optofluidics: A new river of light,” Nat. Photonics 1, 106-114 (2007).

307. D. Psaltis, S. R. Quake, and C. Yang, "Developing optofluidic technology through the fusion of microfluidics and optics," Nature 442, 381-386 (2006).

308. K. I. Petsas, A. B. Coates, and G. Grynberg, "Crystallography of optical lattices," Phys. Rev. A 50, 5173-5189 (1994).

309. A. E. Chiou, W. Wang, G. J. Sonek, J. Hong, and M. W. Berns, "Interferometric optical tweezers," Opt. Commun. 133, 7-10 (1997).

310. M. P. MacDonald, L. Paterson, W. Sibbett, and K. Dholakia, "Trapping and manipulation of low-index particles in a two-dimensional interferometric optical trap," Opt. Lett. 26, 863 (2001).

311. P. Zemánek, V. Karásek, and A. Sasso, "Optical forces acting on Rayleigh particle placed into interference field," Opt. Commun. 240, 401-415 (2004).

312. A. Casaburi, G. Pesce, P. Zemánek, and A. Sasso, "Two-and three-beam interferometric optical tweezers," Opt. Commun. 251, 393-404 (2005).

313. J.-M. Fournier, G. Boer, G. Delacrétaz, P. Jacquot, J. Rohner, and R.-P. Salathé, "Building Optical Matter with Binding and Trapping Forces," in "Optical trapping and optical micromanipulation: Proceedings of SPIE,", vol. 5514, K. Dholakia and G. Spalding, eds. (2004), vol. 5514, pp. 309-317.

314. J.-M. Fournier, J. Rohner, P. Jacquot, R. Johann, S. Mieas, and R.-P. Salathé, “Assembling mesoscopic partices by various optical shemes," in "Optical trapping and optical micromanipulation 2: Proceedings of SPIE,", vol. 5930 K. Dholakia and G. Spalding, eds. (2005), vol. 5930, pp. 59300Y-1 - 59300Y-10.

315. K. Ladavac, K. Kasza, and D. G. Grier, "Sorting mesoscopic objects with periodic potential landscapes: Optical fractionation," Phys. Rev. E 70, 010901 (2004).

316. M. Pelton, K. Ladavac, and D. G. Grier, "Transport and fractionation in periodic potential-energy landscapes," Phys. Rev. E 70, 031108 (2004).

317. I. Ricárdez-Vargas, P. Rodríguez-Montero, R. Ramos-García, and K. Volke-Sepúlveda, "Modulated optical sieve for sorting of polydisperse microparticles," Appl. Phys. Lett. 88, 121116:1-3 (2006).

318. P. Jákl, V. Arzola, M. Šiler, L. Chvátal, K. Volke-Sepúlveda, and P. Zemánek, "Optical sorting of nonspherical and living microobjects in moving interference structures," Opt. Express 22, 29746-29760 (2014).

319. R. L. Smith, G. C. Spalding, K. Dholakia, and M. P. MacDonald, "Colloidal sorting in dynamic optical lattices," J. Opt. A: Pure Appl. Opt. 9, S134-S138 (2007).

320. L. P. Faucheux, L. S. Bourdieu, P. D. Kaplan, and A. J. Libchaber, "Optical thermal ratchet," Phys. Rev. Lett. 74, 1504-1507 (1995).

321. S.-H. Lee, K. Ladavac, M. Polin, and D. G. Grier, "Observation of Flux Reversal in a Symmetric Optical Thermal Ratchet,” Phys. Rev. Lett. 94, 110601 (2005).

322. P. Hänggi and F. Marchesoni, "Artificial Brownian motors: Controlling the transport on the nanoscale," Rev. Mod. Phys. 81, 387-442 (2009).

323. S.-H. Lee and D. G. Grier, "One-dimensional thermal ratchets," J. Phys.: Condens. Matter 17, S3685-S3695 (2005).

324. A. V. Arzola, K. Volke-Sepúlveda, and J. L. Mateos, "Experimental control of transport and current reversals in a deterministic optical rocking ratchet," Phys. Rev. Lett. 106, 168104:1-4 (2011).

325. A. V. Arzola, K. Volke-Sepúlveda, and J. L. Mateos, "Dynamical analysis of an optical rocking ratchet: Theory and experiment," Phys. Rev. E 87, 062910:1-9 (2013).

326. A. V. Arzola, M. Villasante-Barahona, K. Volke-Sepúlveda, P. Jákl, and P. Zemánek, "Omnidirectional transport in fully reconfigurable two dimensional optical ratchets," Phys. Rev. Lett. 118, 138002 (2017).

327. S. R. De Groot and P. Mazur, Non-equilibrium thermodynamics (Dover, New York, 1984).

328. E. Barkai, Y. Garini, and R. Metzler, "Strange kinetics of single molecules in living cells," Physics Today 65, 29-35 (2012).

329. S. Chandrasekhar, "Brownian motion, dynamical friction, and stellar dynamics," Rev. Mod. Phys. 21, 383-388 (1949).

330. J. W. Goodman, "Some fundamental properties of speckle," J. Opt. Soc. Am. 66, 1145-1150 (1976).

331. P. Horak, J.-Y. Courtois, and G. Grynberg, "Atom cooling and trapping by disorder," Phys. Rev. A 58, 3953-3962 (1998).

332. D. Boiron, C. Mennerat-Robilliard, J.-M. Fournier, L. Guidoni, C. Salomon, and G. Grynberg, "Trapping and cooling cesium atoms in a speckle field," Eur. Phys. J. D 7, 373-377 (1999).

333. R. D. L. Hanes, C. Dalle-Ferrier, M. Schmiedeberg, M. C. Jenkins, and S. U. Egelhaaf, "Colloids in one dimensional random energy landscapes," Soft Matter 8, 2714-2723 (2012).

334. K. M. Douglass, S. Sukhov, and A. Dogariu, "Superdiffusion in optically controlled active media," Nat. Photonics 6, 834-837 (2012).

335. F. Evers, C. Zunke, R. D. L. Hanes, J. Bewerunge, I. Ladadwa, A. Heuer, and S. U. Egelhaaf, "Particle dynamics in two-dimensional random-energy landscapes: Experiments and simulations," Phys. Rev. E 88, 022125 (2013).

336. F. Evers, R. Hanes, C. Zunke, R. Capellmann, J. Bewerunge, C. Dalle-Ferrier, M. Jenkins, I. Ladadwa, A. Heuer, R. Castañeda-Priego, and S. Egelhaaf, "Colloids in light fields: Particle dynamics in random and periodic energy landscapes,” Eur. Phys. J. - Spec. Top. 222, 2995-3009 (2013).

337. G. Volpe, G. Volpe, and S. Gigan, "Brownian motion in a speckle light field: Tunable anomalous diffusion and 
selective optical manipulation,” Sci. Rep. 4, 3936 (2014).

338. J. Bewerunge and S. U. Egelhaaf, "Experimental creation and characterization of random potential-energy landscapes exploiting speckle patterns," Phys. Rev. A 93, 013806 (2016).

339. R. D. L. Hanes, M. Schmiedeberg, and S. U. Egelhaaf, "Brownian particles on rough substrates: Relation between intermediate subdiffusion and asymptotic long-time diffusion," Phys. Rev. E 88, 062133 (2013).

340. S. Bianchi, R. Pruner, G. Vizsnyiczai, C. Maggi, and R. Di Leonardo, "Active dynamics of colloidal particles in time-varying laser speckle patterns," Sci. Rep. 6, 27681 EP (2016).

341. G. Volpe, L. Kurz, A. Callegari, G. Volpe, and S. Gigan, "Speckle optical tweezers: micromanipulation with random light fields," Opt. Express 22, 18159-18167 (2014).

342. G. Brugger, L. S. Froufe-Perez, F. Scheffold, and J. Jose Saenz, "Controlling dispersion forces between small particles with artificially created random light fields," Nat. Commun. 6 (2015).

343. J. Rodríguez and D. L. Andrews, "Influence of the state of light on the optically induced interparticle interaction," Phys. Rev. A 79, 022106 (2009).

344. S. Sukhov, K. M. Douglass, and A. Dogariu, "Dipole-dipole interaction in random electromagnetic fields," Opt. Lett. 38, 2385-2387 (2013).

345. J. M. Auñón, C. W. Qiu, and M. Nieto-Vesperinas, "Tailoring photonic forces on a magnetodielectric nanoparticle with a fluctuating optical source," Phys. Rev. A 88, 043817 (2013).

346. M. Sonnleitner, M. Ritsch-Marte, and H. Ritsch, "Attractive optical forces from blackbody radiation," Phys. Rev. Lett. 111, 023601 (2013).

347. A. M. Yao and M. J. Padgett, "Orbital angular momentum: origins, behavior and applications," Adv. Opt. Photonics 3, 161-204 (2011).

348. L. Paterson, M. P. MacDonald, J. Arlt, W. Sibbett, P. E. Bryant, and K. Dholakia, "Controlled rotation of optically trapped microscopic particles," Science 292, 912-914 (2001).

349. K. Ladavac and D. Grier, "Microoptomechanical pumps assembled and driven by holographic optical vortex arrays," Opt. Express 12, 1144-1149 (2004).

350. F. Han, J. A. Parker, Y. Yifat, C. Peterson, S. K. Gray, N. F. Scherer, and Z. Yan, "Crossover from positive to negative optical torque in mesoscale optical matter," Nat. Commun. 9, 4897 (2018).

351. J. A. Rodrigo and T. Alieva, "Freestyle 3D laser traps: tools for studying light-driven particle dynamics and beyond," Optica 2, 812-815 (2015).

352. J. Rodrigo and T. Alieva, "Light-driven transport of plasmonic nanoparticles on demand," Sci. Rep. 6, 33729 (2016).

353. A. Jesacher, S. Fürhapter, C. Maurer, S. Bernet, and M. Ritsch-Marte, "Holographic optical tweezers for object manipulations at an air-liquid surface," Opt. Express 14, 6342-6352 (2006).

354. A. Jesacher, S. Fürhapter, C. Maurer, S. Bernet, and M. Ritsch-Marte, "Reverse orbiting of microparticles in optical vortices," Opt. Lett. 31, 2824-2826 (2006).

355. V. Kajorndejnukul, W. Ding, S. Sukhov, C. Qiu, and A. Dogariu, "Linear momentum increase and negative optical forces at dielectric interface," Nat. Photonics 7, 787-790 (2013).

356. A. Buzas, L. Kelemen, A. Mathesz, L. Oroszi, G. Vizsnyiczai, T. Vicsek, and P. Ormos, "Light sailboats: Laser driven autonomous microrobots," Appl. Phys. Lett. 101, 041111 (2012).

357. L. N. Ng, B. J. Luff, M. N. Zervas, and J. S. Wilkinson, "Propulsion of gold nanoparticles on optical waveguides," Opt. Commun. 208, 117-124 (2002).

358. S. Gaugiran, S. Getin, J. M. Fedeli, and J. Derouard, "Polarization and particle size dependence of radiative forces on small metallic particles in evanescent optical fields. Evidences for either repulsive or attractive gradient forces," Opt. Express 15, 8146-8156 (2007).

359. S. Gaugiran, S. Gétin, J. M. Fedeli, G. Colas, A. Fuchs, F. Chatelain, and J. Dérouard, "Optical manipulation of microparticles and cells on silicon nitride waveguides," Opt. Express 13, 6956-6963 (2005).

360. B. Ahluwalia, P. McCourt, P. Huser, and O. Helles $\varnothing$, "Optical trapping and propulsion of red blood cells on waveguide surfaces," Opt. Express 18, 21053-21061 (2010).

361. C. Pin, J.-B. Jager, M. Tardif, R. Picard, R. Hadji, F. de Fornela, and B. Cluzel, "Optical tweezing using tunable optical lattices along a few-mode silicon waveguide," Lab Chip 18, 1750-1757 (2018).

362. K. Grujic, O. G. Helles $\varnothing$, J. P. Hole, and J. S. Wilkinson, "Sorting of polystyrene microspheres using a Y-branched optical waveguide," Opt. Express 13, 1-7 (2005).

363. X. Xu, G. Wang, W. Jiao, W. Ji, M. Jiang, and X. Zhang, "Multi-level sorting of nanoparticles on multi-step optical waveguide splitter," Opt. Express 26, 29262-29271 (2018).

364. S. Lin, E. Schonbrun, and K. Crozier, "Optical manipulation with planar silicon microring resonators," Nano Lett. 10, 2408-2411 (2010).

365. M. G. Scullion, Y. Arita, T. F. Krauss, and K. Dholakia, "Enhancement of optical forces using slow light in a photonic crystal waveguide," Optica 2, 816-821 (2015).

366. T. Zhu, A. Novitsky, Y. Cao, M. R. C. Mahdy, L. Wang, F. Sun, Z. Jiang, and W. Ding, "Mode conversion enables optical pulling force in photonic crystal waveguides," Appl. Phys. Lett. 111, 061105 (2017).

367. P. J. Reece, V. Garcés-Chávez, and K. Dholakia, "Near-field optical micromanipulation with cavity enhanced evanescent waves," Appl. Phys. Lett. 88, 221116 (2006).

368. A. Yang, S. Moore, B. Schmidt, M. Klug, M. Lipson, and D. Erickson, "Optical manipulation of nanoparticles and biomolecules in sub-wavelength slot waveguides," Nature 457, 71-75 (2009). 
369. B. S. Schmidt, A. H. J. Yang, D. Erickson, and M. Lipson, "Optofluidic trapping and transport on solid core waveguides within a microfluidic device," Opt. Express 15, 14322-14334 (2007).

370. B. Ahluwalia, $\varnothing$. Helle, and O. Helles $\varnothing$, "Rib waveguides for trapping and transport of particles," Opt. Express 24, 4477-4487 (2016).

371. S. Kawata and T. Tani, "Optically driven Mie particles in an evanescent field along a channeled waveguide," Opt. Lett. 21, 1768-1770 (1996).

372. K. Grujic, O. Helles $\varnothing$, J. Wilkinson, and J. Hole, "Optical propulsion of microspheres along a channel waveguide produced by Cs+ ion-exchange in glass," Opt. Commun. 239, 227-235 (2004).

373. A. Yang, T. Lerdsuchatawanich, and D. Erickson, "Forces and transport velocities for a particle in a slot waveguide," Nano Lett. 9, 1182-1188 (2009).

374. K. Grujic and O. G. Helles $\varnothing$, "Dielectric microsphere manipulation and chain assembly by counter-propagating waves in a channel waveguide," Opt. Express 15, 6470-6477 (2007).

375. M. Šiler, T. Čižmár, and P. Zemánek, "Speed enhancement of multi-particle chain in a traveling standing wave," Appl. Phys. Lett. 100, 051103 (2012).

376. M. Soltani, J. Lin, R. A. Forties, J. T. Inman, S. N. Saraf, R. M. Fulbright, M. Lipson, and M. D. Wang, "Nanophotonic trapping for precise manipulation of biomolecular arrays," Nat. Nanotechnol. 9, 448-452 (2014).

377. G. Wang, Z. Ying, H.-P. Ho, Y. Huang, N. Zou, and X. Zhang, "Nano-optical conveyor belt with waveguide-coupled excitation," Opt. Lett. 41, 528-531 (2016).

378. G. Brambilla, G. S. Murugan, J. S. Wilkinson, and D. J. Richardson, "Optical manipulation of microspheres along a subwavelength optical wire," Opt. Lett. 32, 3041-3043 (2007).

379. G. Brambilla, F. Xu, P. Horak, Y. Jung, F. Koizumi, N. P. Sessions, E. Koukharenko, X. Feng, G. S. Murugan, J. S.Wilkinson, and D. J. Richardson, "Optical fiber nanowires and microwires: fabrication and applications," Adv.Opt. Photon. 1, 107-161 (2009).

380. G. Brambilla, “Optical fibre nanowires and microwires: A review," J. Opt. 12, 043001 (2010).

381. A. Maimaiti, D. Holzmann, V. G. Truong, H. Ritsch, and S. Nic Chormaic, "Nonlinear force dependence on optically bound micro-particle arrays in the evanescent fields of fundamental and higher order microfibre modes," Sci. Rep. 6, 30131 (2016).

382. M. Daly, V. G. Truong, and S. N. Chormaic, "Evanescent field trapping of nanoparticles using nanostructured ultrathin optical fibers," Opt. Express 24, 14470-14482 (2016).

383. S. Mandal and D. Erickson, "Optofluidic transport in liquid core waveguiding structures," Appl. Phys. Lett. 90, 184103 (2007).

384. H. Schmidt and A. Hawkins, "Optofluidic waveguides: I. Concepts and implementations," Microfluid. Nanofluid. 4, 3-16 (2008).

385. M. Daly, M. Sergides, and S. Chormaic, "Optical trapping and manipulation of micrometer and submicrometer particles," Las. Phot. Rev. 9, 1-21 (2015).

386. P. Paiè, T. Zandrini, R. Martínez Vázquez, R. Osellame, and F. Bragheri, "Particle Manipulation by Optical Forces in Microfluidic Devices," Micromachine 9, 200 (2018).

387. V. I. Balykin, K. Hakuta, F. Le Kien, J. Q. Liang, and M. Morinaga, "Atom trapping and guiding with a subwavelength-diameter optical fiber," Phys. Rev. A 70, 011401 (2004).

388. G. Sagué, E. Vetsch, W. Alt, D. Meschede, and A. Rauschenbeutel, "Cold-atom physics using ultrathin optical fibers: Light-induced dipole forces and surface interactions," Phys. Rev. Lett. 99, 163602 (2007).

389. V. I. Balykin and V. S. Letokhov, "Atomic cavity with light-induced mirrors," Applied Physics B: Lasers and Optics 48, 517-523 (1989).

390. P. Schneeweiss, S. T. Dawkins, R. Mitsch, D. Reitz, E. Vetsch, and A. Rauschenbeutel, "A nanofiber-based optical conveyor belt for cold atoms," Appl. Phys. B 110, 279-283 (2013).

391. M. Ol'Shanii, Y. Ovchinnikov, and V. Letokhov, "Laser guiding of atoms in a hollow optical fiber," Opt. Commun. 98, 77-79 (1993).

392. M. J. Renn, D. Montgomery, O. V. Vdonin, D. Z. Anderson, C. E. Wieman, and E. A. Cornell, "Laser-Guided Atoms in Hollow-Core Optical Fibers," Phys. Rev. Lett. 75, 3253-3256 (1995).

393. M. Renn, R. Pastel, and H. Lewandowski, "Laser guidance and trapping of mesoscale particles in hollow-core optical fibers," Phys. Rev. Lett. 82, 1574-1577 (1999).

394. R. F. Cregan, "Single-mode photonic band gap guidance of light in air," Science 285, 1537-1539 (1999).

395. P. Russell, "Photonic crystal fibers," Science 299, 358-362 (2003).

396. F. Benabid, J. Knight, and P. Russell, "Particle levitation and guidance in hollow-core photonic crystal fiber," Opt. Express 10, 1195-1203 (2002).

397. O. A. Schmidt, T. G. Euser, and P. S. Russell, "Mode-based microparticle conveyor belt in air-filled hollow-core photonic crystal fiber," Opt. Express 21, 29383-29391 (2013).

398. D. Grass, J. Fesel, S. G. Hofer, N. Kiesel, and M. Aspelmeyer, "Optical trapping and control of nanoparticles inside evacuated hollow core photonic crystal fibers," Appl. Phys. Lett. 108, 221103 (2016).

399. D. S. Bykov, O. A. Schmidt, T. G. Euser, and P. S. J. Russell, "Flying particle sensors in hollow-core photonic crystal fibre," Nature Phot 9, 461-465 (2015).

400. T. Imasaka, Y. Kawabata, T. Kaneta, and Y. Ishidzu, "Optical chromatography," Anal. Chem. 67, 1763-1765 (1995).

401. T. Kaneta, Y. Ishidzu, N. Mishima, and T. Imasaka, "Theory of optical chromatography,” Anal. Chem. 69, 2701-2710 
(1997).

402. J. Makihara, T. Kaneta, and T. Imasaka, "Optical chromatography size determination by eluting particles," Talanta 48, 551-557 (1999).

403. S. J. Hart and A. V. Terray, "Refractive-index-driven separation of colloidal polymer particles using optical chromatography," Appl. Phys. Lett. 83, 5316-5318 (2003).

404. J. D. Taylor, A. Terray, and S. J. Hart, "Analytical particle measurements in an optical microflume," Anal. Chim. Acta 670, 78-83 (2010).

405. J. Burgin, S. Si, M.-H. Delville, and J.-P. Delville, "Enhancing optofluidic actuation of micro-objects by tagging with plasmonic nanoparticles," Opt. Express 22, 10139-10150 (2014).

406. T. Hatano, T. Kaneta, and T. Imasaka, "Application of optical chromatography to imunoassay," Anal. Chem. 69, 2711-2715 (1997).

407. S. Miki, T. Kaneta, and T. Imasaka, "Visualization of an immunological reaction between single antigen and antibody molecules by optical chromatography," Anal. Chim. Acta 404, 1-6 (2000).

408. C. G. Hebert, A. Terray, and S. J. Hart, "Toward label-free optical fractionation of blood - optical force measurements of blood cells," Anal. Chem. 83, 5666-5672 (2011).

409. P. Ashok, R. Marchington, P. Mthunzi, T. Krauss, and K. Dholakia, "Optical chromatography using a photonic crystal fiber with on-chip fluorescence excitation,” Opt. Express 18, 6396-6407 (2010).

410. S. B. Kim, J. H. Kim, and S. S. Kim, "Theoretical development of in situ optical particle separator: cross-type optical chromatography," Appl. Opt. 45, 6919-6924 (2006).

411. C. Helmbrecht, R. Niessner, and C. Haisch, "Photophoretic velocimetry for colloid characterization and separation in a cross-flow setup," Anal. Chem. 79, 7097-7103 (2007).

412. S. I. Rubinow and J. B. Keller, “The transverse force on a spinning sphere moving in a viscous fluid," J. Fluid Mech. 11, 447-459 (1961)

413. G. Cipparrone, R. J. Hernandez, P. Pagliusi, and C. Provenzano, "Magnus force effect in optical manipulation," Phys. Rev. A 84, 015802 (2011).

414. P. G. Saffman, "The lift on a small sphere in a slow shear flow," J. Fluid Mech. 22, 385-400 (1965).

415. Y. Arita, A. W. McKinley, M. Mazilu, H. Rubinsztein-Dunlop, and K. Dholakia, "Picoliter rheology of gaseous media using a rotating optically trapped birefringent microparticle," Anal. Chem. 83, 8855-8858 (2011).

416. N. Descharmes, U. P. Dharanipathy, Z. Diao, M. Tonin, and R. Houdré, "Observation of backaction and self-induced trapping in a planar hollow photonic crystal cavity," Phys. Rev. Lett. 110, 123601 (2013).

417. M. I. Petrov, S. V. Sukhov, A. A. Bogdanov, A. S. Shalin, and A. Dogariu, "Surface plasmon polariton assisted optical pulling force," Laser Photon. Rev. 10, 116-122 (2016).

418. A. Ivinskaya, M. I. Petrov, A. A. Bogdanov, I. Shishkin, P. Ginzburg, and A. S. Shalin, "Plasmon-assisted optical trapping and anti-trapping," Light Sci. Appl. 6, e16258 (2017).

419. A. Maslov, "Resonant pulling of a microparticle using a backward surface wave," Phys. Rev. Lett. 112, 113903 (2014).

420. A. Maslov and B. MI, "Resonant optical gun," Opt. Lett. 39, 2823 (2014).

421. H. Markovich, I. I. Shishkin, N. Hendler, and P. Ginzburg, "Optical Manipulation along an Optical Axis with a Polarization Sensitive Meta-Lens," Nano Lett. 18, 5024-5029 (2018).

422. X. Wang, Y. Dai, Y. Zhang, C. Min, and X. Yuan, "Plasmonic manipulation of targeted metallic particles by polarization-sensitive metalens," ACS Photonics 5, 2945 (2018).

423. P. R. Huft, J. D. Kolbow, J. T. Thweatt, and N. C. Lindquist, "Holographic plasmonic nanotweezers for dynamic trapping and manipulation," Nano Lett. 17, 7920-7925 (2017).

424. P. Gascoyne and J. Vykoukal, "Particle separation by dielectrophoresis," Electrophoresis 23, 1973-1983 (2002).

425. C.-M. Ho, ed., Micro/Nano Technology Systems for Biomedical Applications (Oxford University Press, Oxford, 2010).

426. H.-y. Hsu, A. T. Ohta, P.-Y. Chiou, A. Jamshidi, S. L. Neale, and M. C. Wu, "Phototransistor-based optoelectronic tweezers for dynamic cell manipulation in cell culture media," Lab Chip 10, 165-172 (2010).

427. A. Jamshidi, P. J. Pauzauskie, P. J. Schuck, A. T. Ohta, P.-Y. Chiou, J. Chou, P. Yang, and M. C. Wu, "Dynamic manipulation and separation of individual semiconducting and metallic nanowires," Nat. Photonics 2, 85-89 (2008)

428. D. Pai and B. Springett, "Physics of electrophotography," Rev. Mod. Phys. 65, 163-211 (1993).

429. P. Chiou, A. Ohta, and M. C. Wu, "Massively parallel manipulation of single cells and microparticles using optical images," Nature 436, 370-372 (2005).

430. A. Kumar, S. J. Williams, H.-S. Chuang, N. G. Green, and S. T. Wereley, "Hybrid opto-electric manipulation in microfluidics-opportunities and challenges," Lab on a Chip 11, 2135-2148 (2011).

431. H. Hwang and J.-K. Park, "Optoelectrofluidic platforms for chemistry and biology," Lab on a Chip 11, 33-47 (2011).

432. A. T. Ohta, P.-Y. Chiou, T. H. Han, J. C. Liao, U. Bhardwaj, E. R. B. McCabe, F. Yu, R. Sun, and M. C. Wu, "Dynamic cell and microparticle control via optoelectronic tweezers," J. Microelectromech. Syst. 16, 491-499 (2007).

433. S. Zhang, Y. Liu, Y. Qian, W. Li, J. Juvert, P. Tian, J.-C. Navarro, A. W. Clark, E. Gu, M. D. Dawson, J. M. Cooper, and S. L. Neale, "Manufacturing with light - micro-assembly of opto-electronic microstructures," Opt. Express 25, 28838-28850 (2017).

434. S.-H. Hung, Y.-H. Lin, and G.-B. Lee, “A microfluidic platform for manipulation and separation of oil-in-water 
emulsion droplets using optically induced dielectrophoresis," J. Micromech. Microeng. 20, 045026 (2010).

435. P. J. Pauzauskie, A. Jamshidi, J. K. Valley, J. H. Satcher Jr, and M. C. Wu, "Parallel trapping of multiwalled carbon nanotubes with optoelectronic tweezers," Appl. Phys. Lett. 95, 113104 (2009).

436. M. Hoeb, J. O. Raedler, S. Klein, M. Stutzmann, and M. S. Brandt, "Light-induced dielectrophoretic manipulation of DNA,” Biophysical Journal 93, 1032-1038 (2007).

437. K.-W. Huang, Y.-C. Wu, J.-A. Lee, and P.-Y. Chiou, "Microfluidic integrated optoelectronic tweezers for single-cell preparation and analysis," Lab Chip 13, 3721-3727 (2013).

438. A. H. Jeorrett, S. L. Neale, D. Massoubre, E. Gu, R. K. Henderson, O. Millington, K. Mathieson, and M. D. Dawson, "Optoelectronic tweezers system for single cell manipulation and fluorescence imaging of live immune cells," Opt. Express 22, 1372-1380 (2014).

439. J. K. Valley, A. Jamshidi, A. T. Ohta, H.-Y. Hsu, and M. C. Wu, "Operational regimes and physics present in optoelectronic tweezers," J. Microelectromech. Syst. 17, 342-350 (2008).

440. S. L. Neale, M. Mazilu, J. I. B. Wilson, K. Dholakia, and T. F. Krauss, "The resolution of optical traps created by light induced dielectrophoresis (LIDEP)," Opt. Express 15, 12619-12626 (2007).

441. S. Zhang, N. Shakiba, Y. Chen, Y. Zhang, P. Tian, J. Singh, M. D. Chamberlain, M. Satkauskas, A. G. Flood, N. P. Kherani, S. Yu, P. W. Zandstra, and A. R. Wheeler, "Patterned optoelectronic tweezers: a new scheme for selecting, moving, and storing dielectric particles and cells," Small 14, 1803342 (2018).

442. J. Li, E. H. Hill, L. Lin, and Y. Zheng, "Optical Nanoprinting of Colloidal Particles and Functional Structures," ACS Nano 13, 3783-3795 (2019).

443. D. Braun and A. Libchaber, "Trapping of dna by thermophoretic depletion and convection," Phys. Rev. Lett. 89, 188103 (2002).

444. S. Duhr and D. Braun, "Why molecules move along a temperature gradient," P. Natl. Acad. Sci. USA 103, 19678-19682 (2006).

445. R. T. Schermer, C. C. Olson, J. P. Coleman, and F. Bucholtz, "Laser-induced thermophoresis of individual particles in a viscous liquid," Opt. Express 19, 10571-10586 (2011).

446. S. Duhr and D. Braun, "Optothermal molecule trapping by opposing fluid flow with thermophoretic drift," Phys. Rev. Lett. 97, 038103 (2006).

447. F. M. Weinert and D. Braun, “An optical conveyor for molecules,” Nano Lett. 9, 4264-4267 (2009).

448. L. H. Thamdrup, N. B. Larsen, and A. Kristensen, "Light-induced local heating for thermophoretic manipulation of dna in polymer micro- and nanochannels," Nano Lett. 10, 826-832 (2010).

449. S. Duhr and D. Braun, "Two-dimensional colloidal crystals formed by thermophoresis and convection," Appl. Phys. Lett. 86, 131921 (2005)

450. R. D. Leonardo, F. Ianni, and G. Ruocco, “Colloidal attraction induced by a temperature gradient," Langmuir $\mathbf{2 5}$ 4247-4250 (2009).

451. H.-R. Jiang, H. Wada, N. Yoshinaga, and M. Sano, "Manipulation of colloids by a nonequilibrium depletion force in a temperature gradient," Phys. Rev. Lett. 102, 208301 (2009).

452. Y. Liu and A. W. Poon, "Flow-assisted single-beam optothermal manipulation of microparticles," Opt. Express 18, 18483-18491 (2010).

453. H. Xin, X. Li, and B. Li, "Massive photothermal trapping and migration of particles by a tapered optical fiber," Opt. Express 19, 17065-17074 (2011).

454. V. Garcés-Chávez, R. Quidant, P. J. Reece, G. Badenes, L. Torner, and K. Dholakia, "Extended organization of colloidal microparticles by surface plasmon polariton excitation,” Phys. Rev. B 73, 085417 (2006).

455. A. Cuche, A. Canaguier-Durand, E. Devaux, J. A. Hutchison, C. Genet, and T. W. Ebbesen, "Sorting nanoparticles with intertwined plasmonic and thermo-hydrodynamical forces," Nano Lett. 13, 4230-4235 (2013).

456. G. L. Liu, J. Kim, Y. Lu, and L. P. Lee, “Optofluidic control using photothermal nanoparticles,” Nat. Mater. 5, 27-32 (2006).

457. X. Miao, B. K. Wilson, and L. Y. Lin, "Localized surface plasmon assisted microfluidic mixing," Appl. Phys. Lett. 92, 124108 (2008).

458. J. S. Donner, G. Baffou, D. McCloskey, and R. Quidant, "Plasmon-assisted optofluidics,” ACS Nano 5, 5457-5462 (2011).

459. Z. Kang, J. Chen, S.-Y. Wu, K. Chen, S.-K. Kong, K.-T. Yong, and H.-P. Ho, "Trapping and assembling of particles and live cells on large-scale random gold nano-island substrates," Scie. Rep. 5, 9978 (2015).

460. J. Chen, Z. Kang, S. K. Kong, and H.-P. Ho, "Plasmonic random nanostructures on fiber tip for trapping live cells and colloidal particles," Opt. Lett. 40, 3926-3929 (2015).

461. M. Braun and F. Cichos, "Optically controlled thermophoretic trapping of single nano-objects," ACS Nano 7, 11200-11208 (2013).

462. M. Braun, A. Wurger, and F. Cichos, "Trapping of single nano-objects in dynamic temperature fields," Phys. Chem. Chem. Phys. 16, 15207-15213 (2014).

463. M. Braun, A. P. Bregulla, K. Günther, M. Mertig, and F. Cichos, "Single molecules trapped by dynamic inhomogeneous temperature fields," Nano Lett. 15, 5499-5505 (2015).

464. O. A. Schmidt, M. K. Garbos, T. G. Euser, and P. S. J. Russell, "Reconfigurable optothermal microparticle trap in air-filled hollow-core photonic crystal fiber," Phys. Rev. Lett. 109, 024502 (2012).

465. C. N. Davies, Aerosol science (Academic Press, New York, 1996). 
466. M. Lewittes, S. Arnold, and G. Oster, "Radiometric levitation of micron sized spheres," Appl. Phys. Lett. 40, 455-457 (1982).

467. V. G. Shvedov, C. Hnatovsky, N. Eckerskorn, A. V. Rode, and W. Krolikowski, "Polarization-sensitive photophoresis," Appl. Phys. Lett. 101, 051106 (2012).

468. Y. Yalamov, V. Kutukov, and E. Shchukin, "Theory of the photophoretic motion of the large-size volatile aerosol particle," J. Colloid Interf. Sci. 57, 564 - 571 (1976).

469. M. Pope, S. Arnold, and L. Rozenshtein, "Photophoretic spectroscopy," Chem. Phys. Lett. 62, 589 - 591 (1979).

470. S. Arnold and Y. Amani, "Broadband photophoretic spectroscopy," Opt. Lett. 5, 242-244 (1980).

471. S. Arnold and M. Lewittes, "Size dependence of the photophoretic force,” J. Appl. Phys. 53, 5314-5319 (1982).

472. W. M. Greene, R. E. Spjut, E. Bar-Ziv, J. P. Longwell, and A. F. Sarofim, "Photophoresis of irradiated spheres: evaluation of the complex index of refraction," Langmuir 1, 361-365 (1985).

473. A. S. Desyatnikov, V. G. Shvedov, A. V. Rode, W. Krolikowski, and Y. S. Kivshar, "Photophoretic manipulation of absorbing aerosol particles with vortex beams: theory versus experiment," Opt. Express 17, 8201-8211 (2009).

474. V. G. Shvedov, C. Hnatovsky, N. Shostka, A. V. Rode, and W. Krolikowski, "Optical manipulation of particle ensembles in air," Opt. Lett. 37, 1934-1936 (2012).

475. N. O. Eckerskorn, N. Zeng, V. G. Shvedov, W. Krolikowski, and A. V. Rode, "Effect of polarization on transport of particles in air by optical vortex beam," J. Opt. 14, 055302 (2012).

476. V. G. Shvedov, A. S. Desyatnikov, A. V. Rode, W. Krolikowski, and Y. S. Kivshar, “Optical guiding of absorbing nanoclusters in air," Opt. Express 17, 5743-5757 (2009).

477. V. G. Shvedov, A. V. Rode, Y. V. Izdebskaya, A. S. Desyatnikov, W. Krolikowski, and Y. S. Kivshar, "Selective trapping of multiple particles by volume speckle field," Opt. Express 18, 3137-3142 (2010).

478. V. G. Shvedov, A. V. Rode, Y. V. Izdebskaya, A. S. Desyatnikov, W. Krolikowski, and Y. S. Kivshar, "Giant optical manipulation,” Phys. Rev. Lett. 105, 118103 (2010).

479. V. G. Shvedov, C. Hnatovsky, A. V. Rode, and W. Krolikowski, "Robust trapping and manipulation of airborne particles with a bottle beam,” Opt. Express 19, 17350-17356 (2011).

480. P. Zhang, Z. Zhang, J. Prakash, S. Huang, D. Hernandez, M. Salazar, D. N. Christodoulides, and Z. Chen, “Trapping and transporting aerosols with a single optical bottle beam generated by moiré techniques," Opt. Lett. 36, 1491-1493 (2011).

481. C. Alpmann, M. Esseling, P. Rose, and C. Denz, "Holographic optical bottle beams," Appl. Phys. Lett. 100, 111101 (2012).

482. V. Shvedov, A. R. Davoyan, C. Hnatovsky, N. Engheta, and W. Krolikowski, "A long-range polarization-controlled optical tractor beam," Nat. Photonics 8, 846-850 (2014).

483. V. G. Shvedov, A. V. Rode, Y. V. Izdebskaya, D. Leykam, A. S. Desyatnikov, W. Krolikowski, and Y. S. Kivshar, "Laser speckle field as a multiple particle trap," J. Opt. 12, 124003 (2010).

484. S. J. Ebbens and J. R. Howse, "In pursuit of propulsion at the nanoscale," Soft Matter 6, 726-738 (2010).

485. R. Golestanian, T. B. Liverpool, and A. Ajdari, "Designing phoretic micro- and nano-swimmers," New J. Phys. 9, 126 (2007).

486. E. M. Purcell, "Life at low Reynolds number,” Am. J. Phys. 45, 3-11 (1977).

487. G. Volpe, I. Buttinoni, D. Vogt, H.-J. Kummerer, and C. Bechinger, "Microswimmers in patterned environments," Soft Matter 7, 8810-8815 (2011).

488. J. R. Howse, R. A. L. Jones, A. J. Ryan, T. Gough, R. Vafabakhsh, and R. Golestanian, "Self-motile colloidal particles: From directed propulsion to random walk," Phys. Rev. Lett. 99, 048102 (2007).

489. M. Ibele, T. Mallouk, and A. Sen, "Schooling behavior of light-powered autonomous micromotors in water," Angew. Chem. Int. Edit. 48, 3308-3312 (2009).

490. H.-R. Jiang, N. Yoshinaga, and M. Sano, "Active motion of a Janus particle by self-thermophoresis in a defocused laser beam," Phys. Rev. Lett. 105, 268302 (2010).

491. A. A. Solovev, E. J. Smith, C. C. Bof Bufon, S. Sanchez, and O. G. Schmidt, "Light-controlled propulsion of catalytic microengines," Angew. Chem. Int. Edit. 50, 10875-10878 (2011).

492. J. Palacci, S. Sacanna, A. P. Steinberg, D. J. Pine, and P. M. Chaikin, "Living crystals of light-activated colloidal surfers," Science 339, 936-940 (2013).

493. Y. Hong, M. Diaz, U. M. Córdova-Figueroa, and A. Sen, "Light-driven titanium-dioxide-based reversible microfireworks and micromotor/micropump systems," Adv. Funct. Mater. 20, 1568-1576 (2010).

494. J. Palacci, S. Sacanna, S.-H. Kim, G.-R. Yi, D. J. Pine, and P. M. Chaikin, "Light-activated self-propelled colloids," Philos. T. R. Soc. A 372 (2014).

495. L. Wang, M. N. Popescu, F. Stavale, A. Ali, T. Gemming, and J. Simmchen, “Cu@tio2 Janus microswimmers with a versatile motion mechanism," Soft Matter 14, 6969-6973 (2018).

496. A. P. Bregulla and F. Cichos, "Size dependent efficiency of photophoretic swimmers," Faraday Discuss. 184, 381-391 (2015).

497. S. Nedev, S. Carretero-Palacios, P. Kühler, T. Lohmüller, A. S., L. J. E. Anderson, and J. Feldmann, “An optically controlled microscale elevator using plasmonic Janus particles," ACS Photonics 2, 491-496 (2015).

498. B. Qian, D. Montiel, A. Bregulla, F. Cichos, and H. Yang, "Harnessing thermal fluctuations for purposeful activities: The manipulation of single micro-swimmers by adaptive photon nudging," Chem. Sci. 4, 1420-1429 (2013).

499. A. P. Bregulla, H. Yang, and F. Cichos, "Stochastic localization of microswimmers by photon nudging," ACS Nano 
8, 6542-6550 (2014).

500. I. Buttinoni, G. Volpe, F. Kümmel, G. Volpe, and C. Bechinger, "Active brownian motion tunable by light," J. Phys.: Condens. Matter 24, 284129 (2012).

501. A. Würger, "Self-diffusiophoresis of Janus particles in near-critical mixtures," Phys. Rev. Lett. 115, 188304 (2015).

502. S. Samin and R. van Roij, "Self-propulsion mechanism of active Janus particles in near-critical binary mixtures," Phys. Rev. Lett. 115, 188305 (2015).

503. G. Jékely, J. Colombelli, H. Hausen, K. Guy, E. Stelzer, F. Nédélec, and D. Arendt, "Mechanism of phototaxis in marine zooplankton," Nature 456, 395 EP (2008).

504. W. D. Hoff, M. A. van der Horst, C. B. Nudel, and K. J. Hellingwerf, Prokaryotic Phototaxis (Humana Press, Totowa, NJ, 2009), pp. 25-49.

505. X. Garcia, S. Rafaï, and P. Peyla, "Light control of the flow of phototactic microswimmer suspensions," Phys. Rev. Lett. 110, 138106 (2013).

506. N. Schuergers, T. Lenn, R. Kampmann, M. V. Meissner, T. Esteves, M. Temerinac-Ott, J. G. Korvink, A. R. Lowe, C. W. Mullineaux, and A. Wilde, "Cyanobacteria use micro-optics to sense light direction," eLife 5, e12620 (2016).

507. C. Lozano, B. ten Hagen, H. Löwen, and C. Bechinger, "Phototaxis of synthetic microswimmers in optical landscapes," Nat. Commun. 7, 12828 EP (2016).

508. B. Dai, J. Wang, Z. Xiong, X. Zhan, W. Dai, C.-C. Li, S.-P. Feng, and J. Tang, "Programmable artificial phototactic microswimmer," Nat. Nanotechnol. 11, 1087 EP (2016).

509. D. P. Singh, U. Choudhury, P. Fischer, and A. G. Mark, "Non-equilibrium assembly of light-activated colloidal mixtures," Adv. Mater. 29, 1701328 (2017).

510. S. Ilday, G. Makey, G. B. Akguc, Ö. Yavuz, O. Tokel, I. Pavlov, O. Gülseren, and F. Ö. Ilday, "Rich complex behaviour of self-assembled nanoparticles far from equilibrium," Nat. Commun. 8, 14942 EP (2017).

511. F. Schmidt, B. Liebchen, H. Löwen, and G. Volpe, "Light-controlled assembly of active colloidal molecules," arXiv preprint arXiv: 1801.06868 (2018).

512. M. Leoni, J. Kotar, B. Bassetti, P. Cicuta, and M. C. Lagomarsino, "A basic swimmer at low reynolds number," Soft Matter 5, 472-476 (2009).

513. W. Jiang, D. Niu, H. Liu, C. Wang, T. Zhao, L. Yin, Y. Shi, B. Chen, Y. Ding, and B. Lu, "Photoresponsive soft-robotic platform: Biomimetic fabrication and remote actuation," Adv. Funct. Mater. 24, 7598-7604 (2014).

514. E. Uchida, R. Azumi, and Y. Norikane, "Light-induced crawling of crystals on a glass surface," Nat. Commun. 6, 7310 EP (2015).

515. T. Ikeda, J.-i. Mamiya, and Y. Yu, "Photomechanics of liquid-crystalline elastomers and other polymers," Angew. Chem. Int. Ed. 46, 506-528 (2007).

516. C. Ohm, M. Brehmer, and R. Zentel, "Liquid crystalline elastomers as actuators and sensors," Adv. Mater. 22, 3366-3387 (2010).

517. H. Jiang, C. Li, and X. Huang, "Actuators based on liquid crystalline elastomer materials," Nanoscale 5, 5225-5240 (2013).

518. K. D. Harris, R. Cuypers, P. Scheibe, C. L. van Oosten, C. W. M. Bastiaansen, J. Lub, and D. J. Broer, "Large amplitude light-induced motion in high elastic modulus polymer actuators," J. Mater. Chem. 15, 5043 (2005).

519. M. Rogoz, H. Zeng, C. Xuan, D. S. Wiersma, and P. Wasylczyk, "Light-driven soft robot mimics caterpillar locomotion in natural scale," Adv. Opt. Mater. 4, 1689-1694 (2016).

520. H. Zeng, O. M. Wani, P. Wasylczyk, and A. Priimagi, "Light-driven, caterpillar-inspired miniature inching robot," Macromol. Rapid Commun. 39, 1700224 (2017).

521. H. Zeng, P. Wasylczyk, C. Parmeggiani, D. Martella, M. Burresi, and D. S. Wiersma, "Light-fueled microscopic walkers," Adv. Mater. 27, 3883-3887 (2015).

522. S. Palagi, A. G. Mark, S. Y. Reigh, K. Melde, T. Qiu, H. Zeng, C. Parmeggiani, D. Martella, A. Sanchez-Castillo, N. Kapernaum, F. Giesselmann, D. S. Wiersma, E. Lauga, and P. Fischer, "Structured light enables biomimetic swimming and versatile locomotion of photoresponsive soft microrobots," Nat. Mater. 15, 647-653 (2016).

523. M. J. Jebrail, M. S. Bartsch, and K. D. Patel, "Digital microfluidics: a versatile tool for applications in chemistry, biology and medicine," Lab Chip 12, 2452-2463 (2012).

524. E. Samiei, M. Tabrizian, and M. Hoorfar, "A review of digital microfluidics as portable platforms for lab-on a-chip applications," Lab Chip 16, 2376-2396 (2016).

525. R. M. Lorenz, J. S. Edgar, G. D. M. Jeffries, and D. T. Chiu, "Microfluidic and optical systems for the on-demand generation and manipulation of single femtoliter-volume aqueous droplets," Anal. Chem. 78, 6433-6439 (2006).

526. M. Aas, A. Jonáš, and A. Kiraz, "Lasing in optically manipulated, dye-doped emulsion microdroplets," Opt. Commun. 290, 183-187 (2013).

527. A. Karbalaei, R. Kumar, and H. J. Cho, “Thermocapillarity in microfluidics - A review," Micromachines 7, 13 (2016).

528. Y. S. Ryazantsev, M. G. Velarde, R. G. Rubio, E. Guzman, F. Ortega, and P. Lopez, "Thermo- and soluto-capillarity: Passive and active drops," Adv. Colloid Interface Sci. 247, 52-80 (2017).

529. J.-P. Delville, M. R. de Saint Vincent, R. D. Schroll, H. Chraibi, B. Issenmann, R. Wunenburger, D. Lasseux, W. W. Zhang, and E. Brasselet, "Laser microfluidics: Fluid actuation by light," J. Opt. A: Pure Appl. Opt. 11, 034015 (2009).

530. D. Baigl, "Photo-actuation of liquids for light-driven microfluidics: state of the art and perspectives," Lab Chip 12, 
3637-3653 (2012).

531. C. N. Baroud, J.-P. Delville, F. m. c. Gallaire, and R. Wunenburger, "Thermocapillary valve for droplet production and sorting," Phys. Rev. E 75, 046302 (2007).

532. M. L. Cordero, D. R. Burnham, C. N. Baroud, and D. McGloin, "Thermocapillary manipulation of droplets using holographic beam shaping: Microfluidic pin ball,” Appl. Phys. Lett. 93, 034107 (2008).

533. C. Maggi, F. Saglimbeni, M. Dipalo, F. De Angelis, and R. Di Leonardo, "Micromotors with asymmetric shape that efficiently convert light into work by thermocapillary effects," Nat. Commun. 6, 7855 EP (2015).

534. K. Ichimura, S.-K. Oh, and M. Nakagawa, "Light-driven motion of liquids on a photoresponsive surface," Science 288, 1624-1626 (2000).

535. J. Berná, D. A. Leigh, M. Lubomska, S. M. Mendoza, E. M. Pérez, P. Rudolf, G. Teobaldi, and F. Zerbetto, "Macroscopic transport by synthetic molecular machines," Nat. Mater. 4, 704-710 (2005).

536. D. Yang, M. Piech, N. S. Bell, D. Gust, S. Vail, A. A. Garcia, J. Schneider, C.-D. Park, M. A. Hayes, and S. Picraux, "Photon control of liquid motion on reversibly photoresponsive surfaces," Langmuir 23, 10864-10872 (2007).

537. A. Diguet, R.-M. Guillermic, N. Magome, A. Saint-Jalmes, Y. Chen, K. Yoshikawa, and D. Baigl, "Photomanipulation of a droplet by the chromocapillary effect," Angew. Chem. Int. Ed. 48, 9281-9284 (2009).

538. A. Darhuber and S. Troian, "Principles of microfluidic actuation by modulation of surface stresses," Annu. Rev. Fluid Mech. 37, 425-455 (2005).

539. K. Kotz, K. Noble, and G. Faris, “Optical microfluidics,” Appl. Phys. Lett. 85, 2658-2660 (2004).

540. C. N. Baroud, M. R. de Saint Vincent, and J.-P. Delville, "An optical toolbox for total control of droplet microfluidics," Lab Chip 7, 1029-1033 (2007).

541. E. Verneuil, M. L. Cordero, F. Gallaire, and C. N. Baroud, "Laser-induced force on a microfluidic drop: origin and magnitude," Langmuir 25, 5127-5134 (2009).

542. M. R. d. Saint Vincent, R. Wunenburger, and J.-P. Delville, "Laser switching and sorting for high speed digital microfluidics," Appl. Phys. Lett. 92, 154105 (2008).

543. W. Hu and A. T. Ohta, "Aqueous droplet manipulation by optically induced Marangoni circulation,” Microfluid. Nanofluid. 11, 307-316 (2011).

544. L. Florea, K. Wagner, P. Wagner, G. G. Wallace, F. Benito-Lopez, D. L. Officer, and D. Diamond, "PhotoChemopropulsion - Light-Stimulated Movement of Microdroplets," Adv. Mater. 26, 7339-7345 (2014).

545. V. Pimienta and C. Antoine, "Self-propulsion on liquid surfaces," Curr. Opin. Colloid Interface Sci. 19, 290-299 (2014).

546. S. Kaneko, K. Asakura, and T. Banno, "Phototactic behavior of self-propelled micrometer-sized oil droplets in a surfactant solution," Chem. Commun. 53, 2237-2240 (2017).

547. K. Suzuki and T. Sugawara, "Phototaxis of oil droplets comprising a caged fatty acid tightly linked to internal convection," ChemPhysChem 17, 2300-2303 (2016).

548. D. S. Bykov, S. Xie, R. Zeltner, A. Machnev, G. K. L. Wong, T. G. Euser, and P. S. J. Russell, "Long-range optical trapping and binding of microparticles in hollow-core photonic crystal fibre," Light Sci. Appl. 7, 22 (2018).

549. P. S. Russell, "Photonic-crystal fibers," J. Lightwave Technol. 24, 4729-4749 (2006).

550. D. Richardson, J. Fini, and L. Nelson, "Space-divition multiplexing in optical fibres," Nat. Photonics 7, 354-362 (2013).

551. T. Monro, W. Belardi, K. Furusawa, J. Baggett, N. Broderick, and D. Richardson, "Sensing with microstructured optical fibres," Meas. Sci. Technol. 12, 854-858 (2001).

552. G. S. Murugan, M. Belal, C. Grivas, M. Ding, J. S. Wilkinson, and G. Brambilla, “An optical fiber optofluidic particle aspirator," Appl. Phys. Lett. 105, 101103 (2014).

553. W. Talataisong, R. Ismaeel, S. R. Sandoghchi, T. Rutirawut, G. Topley, M. Beresna, and G. Brambilla, "Novel method for manufacturing optical fiber: extrusion and drawing of microstructured polymer optical fibers from a $3 \mathrm{D}$ printer," Opt. Express 26, 32007-32013 (2018).

554. M. Ploeschner, T. Tyc, and T. Čižmár, "Seeing through chaos in multimode fibres," Nat. Photonics 9, 529-538 (2015).

555. L. Shang, Y. Cheng, and Y. Zhao, "Emerging Droplet Microfluidics," Chem. Rev. 117, 7964-8040 (2017).

556. B. R. Bzdek, R. M. Power, S. H. Simpson, J. P. Reid, and C. P. Royall, "Precise, contactless measurement of the surface tension of picolitre aerosol droplets," Chem. Sci. 7, 274-285 (2016).

557. A. Mitra, B. Deutsch, F. Ignatovich, C. Dykes, and L. Novotny, "Nano-optofluidic detection of single viruses and nanoparticles," ACS Nano 4, 1305-1312 (2010).

558. A. Ivinskaya, N. Kostina, A. Proskurin, M. I. Petrov, A. A. Bogdanov, S. Sukhov, A. V. Krasavin, A. Karabchevsky, A. S. Shalin, and P. Ginzburg, "Optomechanical manipulation with hyperbolic metasurfaces," ACS Photonics 5, 4371-4377 (2018).

559. V.-C. Su, C. H. Chu, G. Sun, and D. P. Tsai, "Advances in optical metasurfaces: fabrication and applications,” Opt. Express 26, 13148-13182 (2018).

560. J. Gargiulo, I. L. Violi, S. Cerrota, L. Chvátal, E. Cortès, E. M. Perassi, F. Diaz, P. Zemánek, and F. D. Stefani, "Accuracy and mechanistic details of optical printing of single au and ag nanoparticles," ACS Nano 11, 9678-9688 (2017).

561. J. Gargiulo, T. Brick, I. Violi, F. Herrere, T. Shibanuma, P. Albella, E. Cortes, F. Requejo, S. Maier, and F. Stefani, "Understanding and reducing photo-thermal forces for the fabrication of au nanoparticle dimers by optical printing," 
Nano Lett. 17, 5747-5755 (2017)

562. L. Ling, H.-L. Guo, X.-L. Zhong, L. Huang, J.-F. Li, L. Gan, and Z.-Y. Li, "Manipulation of gold nanorods with dual-optical tweezers for surface plasmon resonance control," Nanotechnology 23, 215302 (2012).

563. U. Zywietz, A. B. Evlyukhin, C. Reinhardt, and B. N. Chichkov, "Laser printing of silicon nanoparticles with resonant optical electric and magnetic responses," Nat. Commun. 5, 3402 (2014).

564. S. Nedev, S. Carretero-Palacios, P. KÃijhler, T. LohmÃijller, A. S. Urban, L. J. E. Anderson, and J. Feldmann, "An optically controlled microscale elevator using plasmonic Janus particles," ACS Photonics 2, 491-496 (2015).

565. D. Smirnova and Y. S. Kivshar, "Multipolar nonlinear nanophotonics," Optica 3, 1241-1255 (2016).

566. O. Ilic, C. M. Went, and H. A. Atwater, "Nanophotonic Heterostructures for Efficient Propulsion and Radiative Cooling of Relativistic Light Sails,” Nano Lett. 18, 5583-5589 (2018).

567. M. Aspelmeyer, T. J. Kippenberg, and F. Marquardt, "Cavity optomechanics,” Rev. Mod. Phys. 86, 1391-1452 (2014).

568. Z.-Q. Yin, A. A. Geraci, and T. Li, “Optomechanics of levitated dielectric particles,” Int. J. Mod. Phys. B 27, 1330018 (2013).

569. G. Ranjit, M. Cunningham, K. Casey, and A. A. Geraci, "Zeptonewton force sensing with nanospheres in an optical lattice," Phys. Rev. A 93, 053801 (2016).

570. E. Hebestreit, M. Frimmer, R. Reimann, and L. Novotny, "Sensing static forces with free-falling nanoparticles," Phys. Rev. Lett. 121, 063602 (2018).

571. V. Jain, J. Gieseler, C. Moritz, C. Dellago, R. Quidant, and L. Novotny, "Direct Measurement of Photon Recoil from a Levitated Nanoparticle," Phys. Rev. Lett. 116, 243601 (2016).

572. T. M. Hoang, Y. Ma, J. Ahn, J. Bang, F. Robicheaux, Z.-Q. Yin, and T. Li, "Torsional optomechanics of a levitated nonspherical nanoparticle," Phys. Rev. Lett. 117, 123604 (2016).

573. S. Kuhn, A. Kosloff, B. A. Stickler, F. Patolsky, K. Hornberger, M. Arndt, and J. Millen, "Full rotational control of levitated silicon nanorods," Optica 4, 356-360 (2017).

574. Z. Xu and T. Li, "Detecting Casimir torque with an optically levitated nanorod," Phys. Rev. A 96, 033843 (2017).

575. V. Svak, O. Brzobohatý, M. Šiler, P. Ják1, J. Kaňka, P. Zemánek, and S. Simpson, "Transverse spin forces and non-equilibrium particle dynamics in a circularly polarized vacuum optical trap,” Nat. Commun. 9, 5453 (2018).

576. R. Pettit, W. Ge, P. Kumar, D. Luntz-Martin, J. Schultz, L. Neukirch, M. Bhattacharya, and A. Vamivakas, "An optical tweezer phonon laser," Nat. Photonics 13, 402-405 (2019).

577. O. Romero-Isart, A. C. Pflanzer, M. L. Juan, R. Quidant, N. Kiesel, M. Aspelmeyer, and J. I. Cirac, "Optically levitating dielectrics in the quantum regime: Theory and protocols," Phys. Rev. A 83, 013803 (2011).

578. N. Kiesel, F. Blaser, U. Delic, D. Grass, R. Kaltenbaek, and M. Aspelmeyer, "Cavity cooling of an optically levitated submicron particle," Proc. Natl. Acad. Sci. USA 110, 14180-14185 (2013).

579. J. Millen, P. Z. G. Fonseca, T. Mavrogordatos, T. S. Monteiro, and P. F. Barker, "Cavity cooling a single charged levitated nanosphere," Phys. Rev. Lett. 114, 123602 (2015).

580. A. T. M. A. Rahman and P. F. Barker, "Laser refrigeration, alignment and rotation of levitated Yb3+:YLF nanocrystals," Nat. Photonics 11, 634-639 (2017).

581. L. P. Neukirch, E. von Haartman, J. M. Rosenholm, and N. VamivakasA., "Multi-dimensional single-spin nano-optomechanics with a levitated nanodiamond," Nat. Photonics 9, 653-657 (2015).

582. U. Delic, M. Reisenbauer, D. Grass, N. Kiesel, V. Vuletic, and M. Aspelmeyer, "Cavity cooling of a levitated nanosphere by coherent scattering," Phys. Rev. Lett. 122, 123602 (2019).

583. L. Magrini, R. A. Norte, R. Riedinger, I. Marinkovic, D. Grass, U. Delic, S. Groeblacher, S. Hong, and M. Aspelmeyer, "Near-field coupling of a levitated nanoparticle to a photonic crystal cavity," Optica 5, 1597-1602 (2018).

584. E. Pince, S. K. P. Velu, A. Callegari, P. Elahi, S. Gigan, G. Volpe, and G. Volpe, "Disorder-mediated crowd control in an active matter system," Nat. Commun. 7, 10907 (2016).

585. M. Mijalkov, A. McDaniel, J. Wehr, and G. Volpe, "Engineering sensorial delay to control phototaxis and emergent collective behaviors," Phys. Rev. X 6, 011008 (2016). 


\section{CVs}

Pavel Zemánek is a senior researcher, the head of the Department of Microphotonics, the deputy director for research, and the scientific director of Application Laboratories of Advanced Microtechnologies and Nanotechnologies at the Institute of Scientific Instruments of the Czech Academy of Sciences (ISI). He received his M.S. degree in physical electronics and microelectronics from Masaryk University, Brno in 1991 and Ph.D. degree in plasma physics from the same university in 1994. Since 1991 he has been employee at ISI with several stays abroad (e.g. Clarendon Laboratory at the University of Oxford, University of St. Andrews). He also served as the head of the Group of Optical Micromanipulation Techniques at ISI, Department of Coherence Optics, external professor at Masaryk University in Brno and Brno University of Technology, member of the OSA International Council, Coordinator of the International Year of Light in the Czech Republic. PZ has been dealing with frequency stabilizations of lasers, laser physics, laser spectroscopy, atom cooling and trapping, optical and Raman tweezers, optical scalpel, laser induced polymerization of micro-objects, optical sorting, delivery and self-arrangement of microobjects and sub-micrometer size objects, droplet microfluidics and lasers, optical levitation in vacuum. He authored or co-authored 92 peer-reviewed papers and gave more than 80 invited talks at various conferences, workshops or institutions abroad. PZ has been awarded by Josef HlÃąvka Award for young scientist, Otto Wichterle Award for promising scientist from the Czech Academy of Sciences, and Siemens Excellence Award 2013 for the best result of basic research.

Giorgio Volpe is a Lecturer in Physical Chemistry at University College London (UCL) in the UK. He obtained his PhD in Photonics at ICFO âĂŞ The Institute of Photonic Sciences in Barcelona (Spain). Currently, his research group focuses on studying the emergence of non-equilibrium behaviors in biological and light-activated synthetic soft matter for applications in materials science and healthcare. He has published 29 peer-reviewed papers in high-impact journals including in Science, Review of Modern Physics, Nature Communications, Physical Review Letters, Science Advances, Proceedings of the National Academy of Sciences U.S.A. and Nano Letters. He is founder and coordinator of a UCL-wide Cross-disciplinary Network on Soft Materials comprising $>80$ PIs from 6 faculties and $>20$ departments. He is a member of the organizing committee of the Complex Nanophotonics Science Camp, a biennial conference providing networking opportunities for early-career researchers in the field of photonics. He has always been active in popularizing cutting-edge research and has contributed to several open days, science fairs, science weeks and school visits with seminars and demonstrations. He was co-founder and editor of Optic \& Photonics Focus, an independent magazine (active from 2008 to 2013), sponsored by OSA (The Optical Society) and EPS (The European Physical Society), making important developments in the field of optics and photonics accessible to the wider public.

Alexandr Jonáš received the M.S. degree in biophysics from Masaryk University, Brno, Czech Republic, in 1996, and the Ph.D. degree in physical and materials engineering from The Brno University of Technology, Brno, Czech Republic, in 2001. He was a post-doc with the European Molecular Biology Laboratory (Germany), and The University of Texas at Austin (USA) and held research and academic positions with the Czech Academy of Sciences, Institute of Scientific Instruments (Czech Republic), Ko $\tilde{g} \breve{g}$ University (Turkey), and Istanbul Technical University, (Turkey). Currently, he is a Research Scientist with the Institute of Scientific Instruments. His research interests include the development and applications of optical micro-manipulation, microscopy, and spectroscopy techniques for the study of microscopic and mesoscopic soft-matter systems, characterization of novel miniature optical components based on liquids, and analysis of thermophysical properties of supercritical fluids and fluid mixtures.

Oto Brzobohatý is a group leader at the Institute of Scientific Instruments of the Czech Academy of Sciences. He started his scientific carrier in the field of low temperature plasma where he used computer simulation and plasma diagnostic techniques to uncover the important 
role of secondary electrons on the plasma density (and consequently on the plasma processing process). At the end of his $\mathrm{PhD}$ studies he had discovered for himself photonics as one of the most interesting and rewarding disciplines with enormous impact for science, health- care and industry. OB quickly established himself at the international forefront in the field of optical manipulation in the experimental part of fundamental studies of the optical binding force forming so-called soft colloidal matter. Beside experimental skills he mastered also the theoretical modeling of the optical forces and revealed how the morphology of plasmonic nanoparticles affects the way they are optical trapped. He led a small team of researchers that for the first time showed experimentally the surprising nature of the so-called "Tractor beam" - which immediately attracted noticeable interest of the scientific community and popular media. Now, as the leader of the "Levitational Photonics Group", OB focuses with his team on the emerging field of optical cooling of the centre-of-mass motion of optically-levitated nanoparticles in high vacuum. OB authored or co-authored 29 peer-reviewed papers including Nature Photonics, Nature Communications, Light: Science \& Applications, Physical Review Letters, Scientific Reports. 